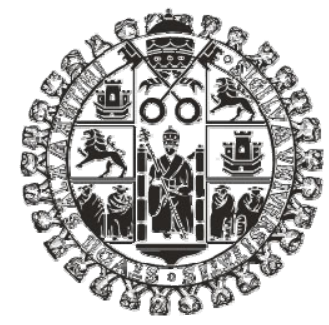

Department of Microbiology and Genetics Institute of Biochemical Microbiology

\title{
Global transcriptional responses of Candida albicans \\ to histone acetyltranferases deletion
}

\author{
Ph.D Thesis \\ Zahra Rashki Ghalehnoo
}

Ph.D Thesis Adviser

Prof. Dr. Angel Dominguez Olavarri

Department of Microbiology and Genetics, University of Salamanca 
Prof. Dr. Angel Dominguez Olavarri Professor and Chair of Microbiology at the Department of Microbiology and Genetics, University of Salamanca

\section{Autoriza:}

That the present dissertation entitled "Global transcriptional responses of Candida albicans to histone acetyltranferases deletion" submitted to apply for Ph.D degree has been carried out by Zahra Rashki Ghalehnoo under my direction in the Department of Microbiology and Genetics, University of Salamanca.

Prof. Dr. Angel Dominguez Olavarri

Professor and Chair 
Prof. Dr. Angel Dominguez Olavarri, Head of the Department of Microbiology and Genetics of the University of Salamanca

\section{Autoriza:}

That the present dissertation entiteld "Global transcriptional responses of Candida albicans to histone acetyltranferases deletion" submitted to apply for Ph.D degree has been carried out by Zahra Rashki Ghalehnoo under direction Prof. Dr. Angel Dominguez Olavarri in the Department of Microbiology and Genetics, University of Salamanca.

Prof. Dr. Angel Dominguez Olavarri Head of the Department of Microbiology and Genetics 
The project work has been supported in part by grants:

DGICYT "Identificación de factores de virulencia y nuevas dianas terapéuticas en Candida albicans mediante análisis funcional del genoma y del proteoma" (Proyecto BIO2002-02124)

Unión Europea. "Interaction of fungal pathogens with host cells: a post genomic approach" dentro del Progama Marie Curie Research Training Network (MRTNCT-2003-504148)

DGICYT "Analisis de las interacciones de Candida albicans con el hospedador: modelos "ex vivo" e "in vivo".

(Proyecto SAF2005-07131)

FIS "Analisis de los mecanismos moleculares de infecciones fungicas mediante aproximaciones genomicas y proteomicas. Establecimiento de modelos animales" (Proyecto PI 050849)

"Glycoshield: surface modulation of the fungal and host response using a genomic approach". Proyectos de investigación en el marco de las redes del Espacio Europeo de Investigación (esquema ERA-NET). GEN2006-27775-C2E/PAT

Junta de Castilla y León "Histona acetyltransferasas en Candida albicans efecto sobre dimorfismo y virulencia. Una aproximación Post-genomica" (SA 141A08) 
In the Name of Allah, the Beneficent, the Merciful

All praise is due ta Allah, wha created the heavens and the earth and made the darkness and the light

Haly Qur'n 


\section{Acknowledgements}

Over these last few years I have come to owe so much to so many people. Therefore I would like to express my sincere gratitude to all the people who have supported me in my work. It is impossible to mention everyone here, but I would like to give special thanks to the following people:

I consider myself extremely lucky to have had Prof. Dr. Angel Dominguez Olavarri for his supervision during my research, for the opportunity to work in his lab, for his thoughtful nature, patient disposure and his presence for whenever I needed his.

I also thank to Prof. Dr. Maria Carmen Lopez for her encouragement and her suggestions.

All people of the group (past and present) are acknowledged for their help, special thanks to Dr. Nuria Martin for her great patience in answering all the questions of a beginner.

I thank the members of my department, who were always warm, kind and helpful and my friends, especially thanks is due to Laura Mojardin for all the help and her friendly support. Her involvement with every aspect of my life makes my every day so much easier.

I also wish to thank all my friends and colleagues at IMb and CSIC. Special thanks are also due to Dr. Mar Sanchez, Dr. Maria Gomez, Dr. Cristina Cotobal, Elisa de Castro, Nazaret Reveron, Dr. Laura Marin, Joana Sequeira for their friendly support.

I would like to thank Dr. Carmen Castro for her helpful technical assistance.

I am very grateful to Dr. Alberto Gonzalez-Novo for providing plasmid, effective and intelligent suggestions.

My acknowledgements will not be complete if I do not mention my father and my mother whom I owe the gift of life. They make me happy to no end, to know of their pride for me having achieved my goal. I am grateful for their kind words, total support and unconditional love.

Finally appreciation and praise is due to my daughters, Mahsa and Rozhan, and especially my husband because nothing would be possible without their support. 


\section{INDEX}

\section{Acknowledgements}

\section{Chapter I Introduction}

General characteristic of C. albicans 1

Macroscopic and microscopic features 1

The genome of $C$. albicans 3

Genetic manipulation with C. albicans 5

Interest of study in the genome profile of Candida 6

albicans morphogenesis

Relation between morphology and cell cycle $\quad 7$

Type of pathogenesis caused by Candida albicans 9

Virulence factors of C. albicans 10

Environmental cues regulate transcription factors 11

Components of the cell signalling pathways 13

Chromatin structure and nucleosomes 16

Histone acetyltransferase complexes 17

Histone acetyltransferase A (GCN5) 20

Histone acetyltransferase B (HAT1 and HAT2) 20

Are type B histone acetyltransferases cytoplasmic 23

or nuclear?

Histone deacetylase complexes 24

The Sin3-histone deacetylase (HDAC) corepressor 25

The cDNA microarray analysis of differencial gene 26

expression in C. albicans

Chapter II Global tarnscriptional responses of Candida

albicans to histone acetyltransferases deletion

Chapter III Phenotypic, virulence and transcriptome analysis

$\begin{array}{lll}\text { Chapter IV Spanish summary } & 111\end{array}$

$\begin{array}{lr}\text { References } & 149\end{array}$ 
Candida albicans, the major human fungal pathogen, undergoes a reversible morphological transition from single yeast cells to pseudohyphal and hyphal filaments (elongated cells attached end-to-end). Because typical $C$. albicans infections contain a mixture of these morphologies it has, for many years, been difficult to assess the relative contribution of each form to virulence. In addition, the regulatory mechanisms that determine growth in pseudohyphal and hyphal morphologies are largely unknown (Biswas et al., 2007; Carlisle et al., 2009).

Fungi are classified on the basis of their ability to reproduce sexually, asexually, or by a combination of both. Thus, $C$. albicans has traditionally been classified in the Deuteromycota (fungi imperfecti) since the sexual phase of this fungus is unknown, although recent reports described the presence of mating-type-like orthologs (MTL) in C. albicans of both of the Saccharomyces cerevisiae mating-type genes (MAT), a and $\alpha$ (Hull \& Johnson, 1999; Magee \& Magee, 2000). Therefore, C. albicans has been classified as follows:

\begin{tabular}{|c|c|}
\hline Kingdom: ..... & .. Fungi \\
\hline Phylum: .. & ...Ascomycota \\
\hline Subphylum: & Ascomycotina \\
\hline Class: ............ & ...Ascomycetes \\
\hline Order: ....... & ..Saccharomycetales \\
\hline Family: & ..Saccharomycetaceae \\
\hline nus: ..... & ..Candida \\
\hline
\end{tabular}

\section{Macroscopic and microscopic features}

The colonies of Candida spp. are cream coloured to yellowish, grow rapidly and mature in three days (Fig. 1.1). The texture of the colony may be pasty, smooth, glistening or dry, wrinkled and dull, depending on the species (Aridogan et al., 2005; Buschelman et al., 1999).

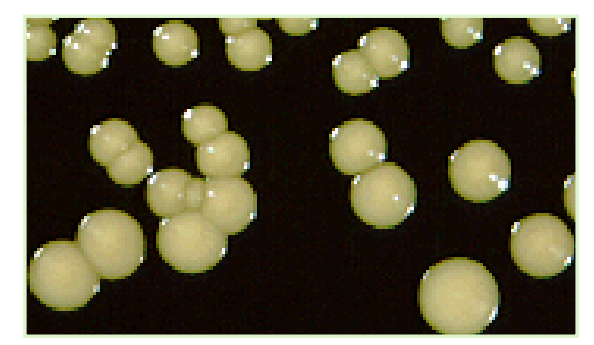

Figure 1.1 Macroscopic features of C. albicans 
C. albicans is able to grow in two different ways; reproduction by budding, forming an ellipsoid bud, and in hyphal form, which can periodically fragment and give rise to new mycelia, or yeast-like forms (Kurzai et al., 2005; Molero et al., 1998; Sanchez-Martinez \& Perez-Martin, 2001). Several factors have been identified that contribute, either positively or negatively for the transition from the yeast to hypha forms (Braun et al., 2001). Many of these factors were identified on the basis of their homology to factors required for diploid pseudohyphal growth of S. cerevisiae (Khalaf \& Zitomer, 2001). Transitions between the two phenotypes can be induced in vitro in response to several environmental cues such as $\mathrm{pH}$, temperature or different compounds such as $\mathrm{N}$-acetyl glucosamine or proline (Molero et al., 1998).

In the laboratory, environmental conditions influence the morphological state of $C$. albicans. Serum causes blastospores to develop true hypha. High temperature $\left(37^{\circ} \mathrm{C}\right)$, high ratio of $\mathrm{CO}_{2}$ to $\mathrm{O}_{2}$, neutral $\mathrm{pH}$ and nutrient-poor media also stimulate hyphal growth. Conversely, low temperatures, air, acidic $\mathrm{pH}$, and enriched media promote blastospore growth (Cutler, 1991; Soares et al., 2008).

Intermediate conditions can induce various pseudohyphal forms as well as true hypha (Fig. 1.2B and C). The transition from a commensally to pathogenic lifestyle may also involve changes in environmental conditions and dispersion within the human host. Although progress has been achieved in recent years, the molecular mechanisms governing these morphogenetic conversions are still not fully understood (Biswas et al., 2007; Kurtz et al., 1988). C. albicans can also be induced to undergo a complex morphological transition to form chlamydospores (Gow et al., 2002; Odds et al., 1988), which are generally defined as thick-walled asexual spores that are derived from a hyphal cell (Fig. 1.2D).
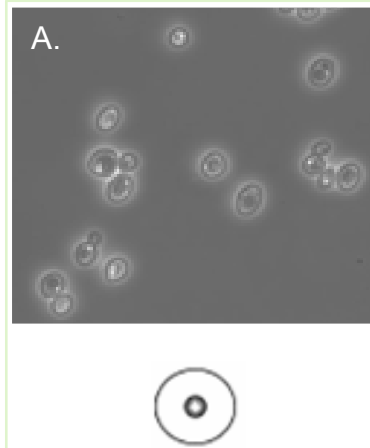
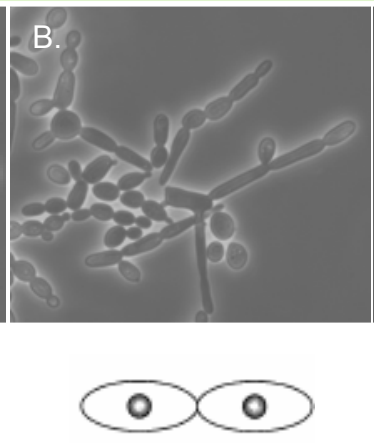
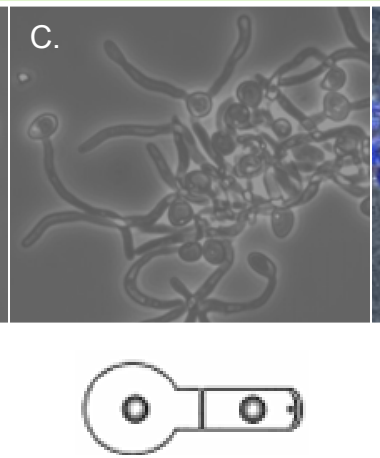
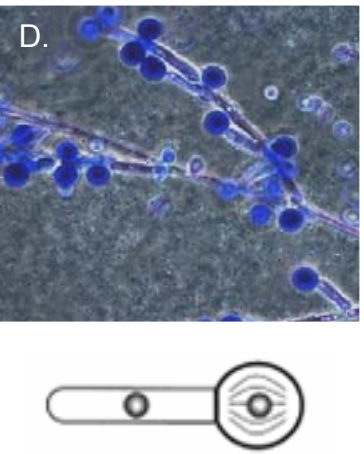

Figure 1.2 Growth of C. albicans yeast (A), pseudohypha (B), hypha (C) and chlamydospore (D)

The role of chlamydospores in the $C$. albicans life cycle is not understood, as they are rarely observed at sites of infection, and there is no evidence that they confer long-term 
viability (Jansons \& Nickerson, 1970; Martin et al., 2005). However, a specific unknown role is suggested by the fact that the only Candida species that form chlamydospores are C. albicans and Candida dubliniensis. In fact, chlamydospore formation is an important diagnostic tool for distinguishing between different Candida species, since the majority of $C$. albicans clinical isolates retain the ability to form chlamydospores (AlHedaithy \& Fotedar, 2002; Al Mosaid et al., 2003; Nobile et al., 2003). These observations raise the possibility that this conserved process contributes an advantage for growth as either a commensal or a pathogen in human hosts. Early studies of chlamydospores in C. albicans were limited to descriptions of their growth, development, and ultrastructural features (Acikgoz et al., 2004; Fotedar \& Al Hedaithy, 2004; Martins-Nishikawa et al., 2002; Odds et al., 1998; Silva et al., 2003; Tekeli et al., 2005; Yang et al., 2003). The empirically determined conditions that favour chlamydospore production were found to include growth in the dark, microaerophilic conditions, room temperature, and nutrient-poor media containing complex carbohydrates. These conditions first trigger cells to grow in long filamentous chains; elongated suspensor cells then develop by branching off of the filaments, and then large rounded chlamydospores ( 8 to $12 \mu \mathrm{m}$ in diameter) form at the end of the suspensor cells. In some cases, a chlamydospore can also form at the terminus of a filament. The mature chlamydospores contain a nucleus and other organelles but are distinguished by the presence of large lipid droplets (Kues, 2000), high RNA content (Vidotto et al., 1996), and a thick outer layer of cell wall that is contiguous with the suspensor cell (Jansons \& Nickerson, 1970).

\section{The genome of Candida albicans}

Candida albicans, one of the first eukaryotic pathogens selected for genome sequencing, is the most commonly encountered human fungal pathogen, causing skin and mucosal infections in generally healthy individuals and life-threatening infections in persons with severely compromised immune function.

The many clinical isolates of $C$. albicans used for laboratory study and genetic analysis are generally diploid and exhibit considerable natural heterozygosity and some have translocations in their genomes (Calderone et al., 2000; Chibana et al., 2000). Although mating governed by a mating-type-like locus can occur, a meiotic phase for the sexual cycle remains obscure and, unlike most species chosen for sequencing, a haploid or homozygous form for C. albicans is not available (Hull et al., 2000; Tzung et al., 2001). Strain SC5314 (Fonzi \& Irwin, 1993), was chosen for large-scale sequencing because 
of its widespread and increasing use in molecular analyses, virulence in animal models, and apparent standard diploid electrophoretic karyotype.

The Candida Genome Sequencing Project started in 1996 and in 2004, it produced a diploid assembly constructed from 10.9x coverage (Assembly 19), which provided single contigs where heterozygosity was not obvious and allelic contigs where there was significant heterozygosity (Jones et al., 2004). The first was the construction of a physical map of one chromosome, chromosome 7 (Chibana et al., 1994). Nucleotide sequence data for Assemblies 5, 6 and 19 of the C. albicans strain SC5314 genome sequence were retrieved from the Stanford Genome Technology Center (SGTC) website (http://www-sequence.stanford.edu/group/candida/) (d'Enfert et al., 2005). CandidaDB (http://genodb.pasteur.fr/CandidaDB) was established in 2002 to provide the first genomic database for the human fungal pathogen Candida albicans.

The new version of CandidaDB houses the latest versions of the genomes of $C$. albicans strains SC5314 and WO-1 along with six genome sequences from species closely related to C. albicans that all belong to the CTG clade of Saccharomycotina Candida tropicalis, Candida (Clavispora) lusitaniae, Candida (Pichia) guillermondii, Lodderomyces elongisporus, Debaryomyces hansenii, Pichia stipitis and the reference Saccharomyces cerevisiae genome (Rossignol et al., 2008).

CandidaDB includes sequences coding for 5417 proteins with annotations collected from other databases, enriched with illustrations of structural features and functional domains and data of comparative analyses. In order to take advantage of the integration of multiple genomes in a unique database, new tools using pre-calculated or user defined comparisons have been implemented that allow rapid access to comparative analysis at the genomic scale (Rossignol et al., 2008). The main facts and figures of the $C$. albicans genome sequence are as follows. Eight chromosomes (historically named 1-7 and R) constitute a haploid genome size of 14,851 kilobases $(\mathrm{kb})$, containing 6,419 open reading frames (ORFs) longer than 100 codons, of which some $20 \%$ have no known counterpart in other available genome sequences. The codon CUG, which is translated abnormally by $C$. albicans as serine rather than leucine, is found at least once in approximately two-thirds of ORFs (Odds et al., 2004). Assembly 19 , the diploid assembly of the genome of C. albicans strain SC5314 was a very important achievement. It provided a great deal of insight into many aspects of genomic organization, especially the large amount of heterozygosity (Newport \& Nejentsev, 2004). The subsequent annotation of the assembly by the community demonstrated a number of important properties of the genome, including the number of genes $(6,354)$, the number with introns $(224)$, the frequency and characteristics of short 
tandem repeats, and the characteristics of several multigene families (Braun et al., 2005), also identified putative spurious genes and genes either on overlapping contigs or truncated by the end of contigs. However, they did not address chromosome location nor try to join the 266 haploid contigs of Assembly 19 into chromosome-sized assemblies. Thus, although these two projects brought the $C$. albicans genome to a very useful state, they still left it incomplete, lacking chromosome-size contigs and with some genes in an ambiguous state. Subsequently, Chibana et al (Chibana et al., 2005) completed the sequence of chromosome 7 , identified 404 genes, and compared the synteny to the $S$. cerevisiae genome. The completed Assembly 21 contains $15.845 \mathrm{Mb}$ of DNA. The assembly does not include the complete telomeric sequences for every chromosome, and includes only one copy of the normally repeated rDNA on chromosome R (Braun et al., 2005). The ORF analysis of Assembly 21 is being carried out at the Candida Genome Database (van het Hoog et al., 2007).

\section{Genetic manipulation with C. albicans}

C. albicans has a nuclear genome of about $16 \mathrm{Mb}, 33 \%$ larger than that of $S$. cerevisiae. Many genes are conserved between S. cerevisiae and C. albicans, and based on those similarity the mechanisms of many biological processes in $C$. albicans have been proposed. In many cases, although the specific components of relevant signalling pathways are conserved, molecular mechanisms and environmental signals have often diverged, most likely because of the coevolution of $C$. albicans and its human host. C. albicans poses special problems for scientists interested in studying gene function because it is diploid and because CUG is translated into a serine instead of a leucine (Kurtz et al., 1988). To analyze the function of a gene, both alleles must be disrupted. Although methods of transformation (spheroplast-polyethylene glycol, lithium acetate and electroporation) are well established, the transformation efficiency is poor. For the study of gene function in $C$. albicans, a number of disruption protocols and selectable markers are commonly utilized (Tournu et al., 2005).

The most widely used marker is the URA3 gene, which encodes Orotidine-5'phosphate decarboxylase that confers uracil prototrophy. However, this marker must be used with caution; URA3 expression levels can affect virulence and are susceptible to chromosome position effects, thereby complicating the analysis of strains constructed with URA3 as a selectable marker (Cheng et al., 2003; Sharkey et al., 2005; Staab et al., 2003). To avoid the use of the URA3 marker, various other auxotrophic markers, as well as dominant markers, have been developed and are 
currently used (Morschhauser et al., 2005; Noble \& Johnson, 2005; Reuss et al., 2004; Shen et al., 2005).

To study the functions of essential genes, the C. albicans MET3 promoter, which is regulated by the level of methionine and/or cysteine in the medium, or the tetracycline on/off system, can be used. These systems allow conditional expression so that the consequences of depletion of a gene product may be investigated (Care et al., 1999; Park \& Morschhauser, 2005; Saville et al., 2003). In order to study the expression of certain genes at either the RNA level or the protein level, a number of $C$. albicansoptimized reporters construct, or tags, have been generated (Berman \& Sudbery, 2002). The development of these molecular tools has greatly accelerated the elucidation of morphogenesis and pathogenesis in C. albicans.

\section{Interest of study in the genome profile of Candida albicans morphogenesis}

The fact that the fungi are eukaryotic organisms with simple and fast development and that are easier to manipulate than pluricellular organisms have stimulated the use of some species of these organisms as model of genetic studies in molecular biology. Morphogenesis can be defiened as the transition between unicellular yeast cells to a filamentous growth form.

With regard to morphogenesis, there is a long history of attempts to prove a relationship between the filamentous form of $C$. albicans and virulence. One of the most interesting features of the $C$. albicans genome is the occurrence of numeric and structural chromosomal rearrangements as means of generating genetic diversity, named chromosome length polymorphisms (contraction/expansion of repeats), reciprocal translocations, chromosome deletions and trisomy of individual chromosomes. These karyotypic alterations lead to changes in the phenotype, which is a strategy of adaptation the fungus.

The availability of the genome sequence has paved the way for the implementation of post-genomic approaches to the study of $C$. albicans: macroarrays and then microarrays have been developed and used to study the C. albicans transcriptome; proteomics has also been developed and complements transcriptional analyses; furthermore, systematic approaches are becoming available to study the contribution of each $C$. albicans gene in different contexts. The genome of $C$. albicans is highly dynamic and this variability has been used advantageously for molecular epidemiological and population studies in this species. A remarkable discovery that has arisen from the genome sequence is the presence of a parasexual cycle in $C$. albicans. This parasexual cycle is under the control of mating-type loci and switching between 
white and opaque phenotypes. Investigating the role that the mating process plays in the dynamics of the $C$. albicans population or in other aspects of $C$. albicans biology and pathogenicity will undoubtedly represent an important focus for future research (Rossignol et al., 2008). Additional interest in the molecular mechanisms of C. albicans morphopathogenic determinants originated from the necessity of identifying new drug targets due to increased drug resistance in clinical isolates.

There is hope that recently developed techniques of manipulating $C$. albicans and the sequencing of its whole genome will lead to a thorough understanding of its virulence and biology, thus offering the possibility of a knowledge-based approach to the development of novel antifungal agents. A major strategy for determining virulence genes as molecular targets for antifungal drugs and vaccines is to identify a specific biochemical or structural target unique to $C$. albicans (or to fungi in general) in an attempt to specifically and selectively disrupt them and determine their effects on virulence.

\section{Relation between morphology and cell cycle}

The cell cycle and its role in morphogenesis in C. albicans remain uncertain. However, some factors involved in the cell cycle have been reported to influence hyphal morphology. One of three homologues of $\mathrm{G} 1$ cyclin, $\mathrm{Ccn} 1 \mathrm{p}$, is necessary to maintain hyphal growth under certain conditions (Loeb et al., 1999a; Loeb et al., 1999b). Another $\mathrm{G} 1$ cyclin homologue, Hgc1p, is essential for hyphal formation but not required for the expression of HSGs (Zheng et al., 2004). The other G1 cyclin homologue, Cln3p, is not only essential for budding, but negatively regulates yeast-hypha transition (Bachewich \& Whiteway, 2005).

The two homologues of $\mathrm{B}$ type cyclins, $\mathrm{Clb} 2 \mathrm{p}$ and $\mathrm{Clb} 4 \mathrm{p}$, affect $\mathrm{C}$. albicans morphogenesis by negatively regulating polarized growth (Bensen et al., 2005). While cell elongation of pseudohyphal growth in $S$. cerevisiae is regulated by a change in the cell cycle (Kron et al., 1994), control of hyphal elongation in C. albicans appears to be independent of the cell cycle, as shown by several lines of evidence. The timing of cell cycle-regulated events, including the phosphorylation state of the tyrosine-18 of the $C$. albicans Cdc28p, spindle pole body duplication, spindle elongation, rearrangement of the actin cytoskeleton, actin structure, chitin ring formation and nuclear division are identical between synchronous yeast and hyphal apical cells (Hazan et al., 2002) (Fig. 1.3). 


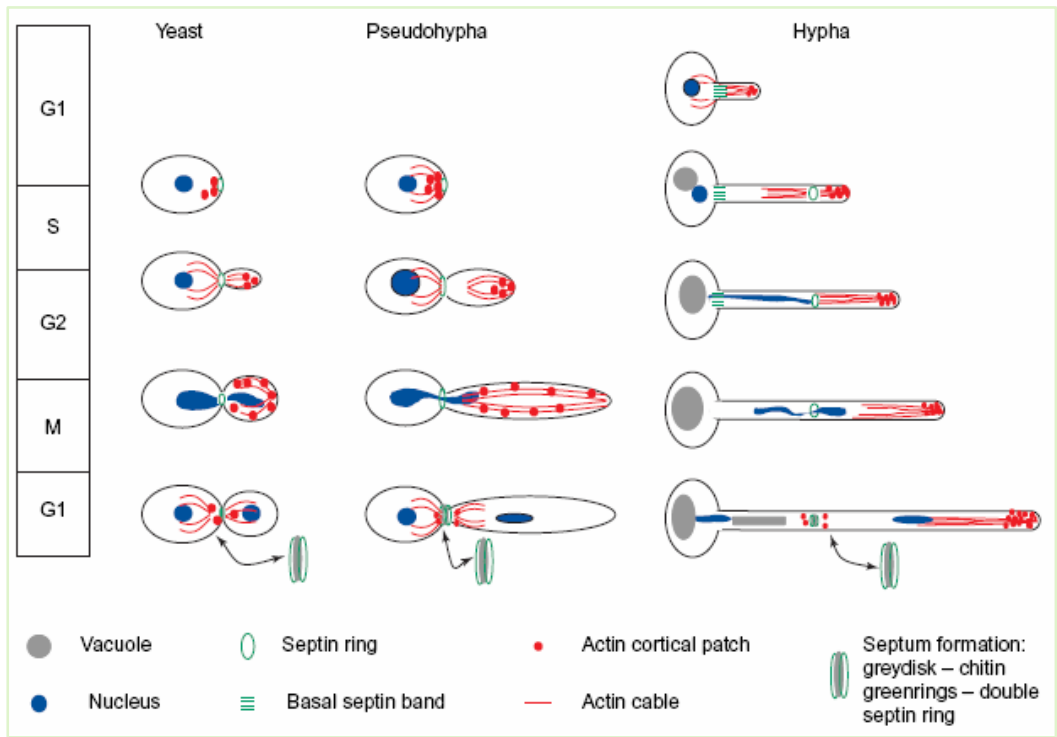

Figure 1.3 Representations of the cell cycles of yeast, pseudohypha and hypha (Sudbery et al., 2004)

Several observations have reported that perturbations of the cell cycle induced a polarized growth of $\mathrm{C}$. albicans. Deletion of $\mathrm{CaFKH2}$, a homologue of the transcription factor FKH2, caused pseudohyphal growth (Bensen et al., 2002). Repressing the G1 cyclin CIn3p under yeast growth conditions caused the yeast cells to arrest in $\mathrm{G} 1$, increase in size and then develop into hypha and pseudohypha (Bachewich \& Whiteway, 2005). Cells depleted of the essential B-type cyclin Clb2p grew highly elongated filaments, while the deletion of the nonessential B-type cyclin Clb4p showed constitutive pseudohyphal growth (Bensen et al., 2005). Cells lacking the cell cycle regulatory polo-like kinase CDC5 formed hyphal-like filaments under yeast growth conditions (Bachewich et al., 2003). The essential gene MCM1 was identified as one of the Candida genes that were able to interfere with FLO11 regulation, which is required for pseudohyphal development, over-expresstion of the gene in S. cerevisiae, and the depletion of CaMcm1p leads to constitutive induction of hypha (Rottmann et al., 2003). Studies from $S$. cerevisiae indicate that cell morphogenesis may involve cell cycle regulation by cyclin-dependent kinase. C. albicans cells can switch from unicellular yeast growth to an alternate growth program that generates linear chains of elongated cells with no constrictions at the site of the septa (Hazan et al., 2002). During hyphal growth in C. albicans, cell surface expansion is restricted to a small region at the hyphal tip. This apical growth zone is active during the entire hyphal growth period (Staebell \& Soll, 1985). In contrast, yeast-form cells expand from a small area in a mostly apical manner only at the initial stage of budding. When the bud has reached a critical size, apical growth shuts down and general (isotropic) expansion takes place (Staebell \& Soll, 1985). The localization of the actin cytoskeleton in yeast and hyphal 
cells reflects these differences in morphogenesis. Polarization of the actin cytoskeleton to the hyphal tip is observed in all hyphal cells. However, in yeast-form cells, the cortical actin patches are observed at the area of apical expansion only in small budded cells but not in large budded cells (Anderson \& Soll, 1986). It has been suggested that the actin cytoskeleton is essential for polarized apical growth because chloropropham, a drug affecting actin microfilament organization, has been shown to inhibit hyphal growth (Yokoyama et al., 1994).

\section{Types of pathogenesis caused by Candida albicans}

According to Odds (Odds et al., 1987), most people usually carry a single strain of Candida at different body sites for a long time, for instances Candida dubliniensis can be found in the oropharynx or in the upper respiratory tract (Sullivan et al., 2005), while C. albicans is a normal component of the oral cavity (Barros et al., 2008), the gastrointestinal tract, and the vaginal environment (Chabrier-Rosello et al., 2008; Pereira-Cenci et al., 2008), but this organism is also an opportunistic pathogen, commonly causing infections such as denture stomatitis, thrush, and urinary tract infections. C. albicans can also cause more serious systemic infections. Often these infections are transmitted in hospitals and are much more common in immunocompromised patients. C. albicans infection rates have increased by over a factor of twenty during the past two decades, in a large part resulting from an increase in immunocompromised persons. Mortality associated with systemic Candida infections is approximately 35-50\% (Darwazeh et al., 1990; Wey et al., 1988). Approximately one half of hospital-acquired C. albicans infections are associated with biofilms formed on an implanted medical device, such as a central venous catheter, urinary catheter (Dominic et al., 2007; Trofa et al., 2008), endotracheal tube, prosthetic heart valve, pacemaker, or joint replacement. These devices provide a route through the body's barrier defences and also provide a surface for cell growth and development (Huth et al., 2009; Opilla, 2008). Furthermore, oropharyngal candidiasis occurs in approximately $70 \%$ of patients with AIDS and $70 \%$ of all women (with or without AIDS) will experience at least one episode of vaginitis caused by Candida and $20 \%$ will experience recurrent disease.

\section{Virulence factors of C. allbicans}

It is generally accepted now that dimorphism, i.e. the ability to grow in a yeast and a elementals growth form, not only is a virulence trait per se, but is also co-regulated with 
other virulence factors, which are associated with cellular morphology (Hube, 1996; White \& Agabian, 1995).

The $C$. albicans hyphal form is often found at sites of tissue invasion, and cells that do not readily form hypha often have reduced virulence (Odds et al., 1988). Importantly, other Candida spp. that does not readily form true hypha are much less frequently isolated from the human host, indicating that they are less virulent (Braun et al., 2000). It is generally thought that hyphal cells expressing cell-wall proteins that facilitate adhesion to human tissues are important for tissue invasion, as well as for escape from phagocytosis mediated by neutrophils or macrophages (Fig. 1.4). By contrast, the yeast form is thought to be important for dissemination of the pathogen through the blood stream. It is likely, therefore, that the ability to switch between the morphological forms is important for $C$. albicans virulence.

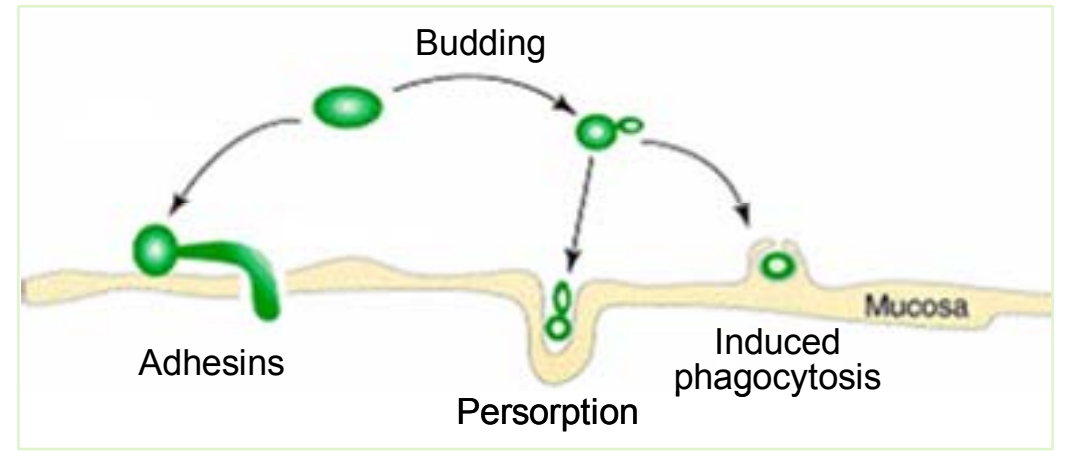

Figure 1.4 Early events in the pathogenesis of candidiasis are portrayed on a mucosal surface. A yeast cell of Candida albicans is shown either budding (right) or germinating (left). At the mucosal surface, germination of yeast cells (left) and penetration of the mucosa is shown.

Virulence depends on opposing reactions between host and pathogen and is intrinsically linked to the host immune status. Virulence factors rely upon microbial attributes that mediate cell damage. Activity of several C. albicans hydrolytic enzymes is well characterized. For example extracellular lipase released by $C$. albicans directly induced cytotoxicity and promoted the deposition of lipid droplets in the cytoplasm of macrophages and hepatocytes (Paraje et al., 2008). Additionally, a membrane protein $(\alpha-1,2$-mannosyltransferase) that is required for both $\mathrm{O}$ and $\mathrm{N}$-mannosylation in fungi might be required for host recognition. Molecules involved in host cell recognition found in Candida are adhesions that bind to several extracellular matrix proteins of mammalian cells, such as fibronectin, laminin, fibrinogen and collagen. Adhesion function (e.g. to human buccal epithelial cells) is provided by agglutinin-like sequences of Candida, resembling the $S$. cerevisiae $\alpha$-agglutinin protein that is required for cellcell recognition during mating. Another potential adherence protein of Candida is reminiscent of the integrin family of mammalian cell receptors. Enzymes that contribute 
to invasiveness are the secreted aspartyl proteinases and phospholipases which form rather large families in C. albicans (Haynes, 2001).

In fact, its ability to adhere to host tissues, produce secretory aspartyl protease and phospholipase enzymes and transform from yeast to hyphal phase are the major determinants of its pathogenicity.

\section{Environmental cues regulate transcription factors}

C. albicans morphology is directly related to environmental conditions. Hyphal development depends on two factors: (i) the nature, number and intensity of environmental signals (outside cues) and (ii) the activity of signalling pathways including key transcription factors (cellular-response machinery). According to current models, environmental cues trigger separate signal-transduction pathways, which regulate common targets required to initiate hyphal growth (Fig. 1.5). A threshold level of signalling may be reached either by simultaneous low-level stimulation of several pathways or by essentially a single pathway, which is fully activated (or hyperactive as in over-expression experiments).

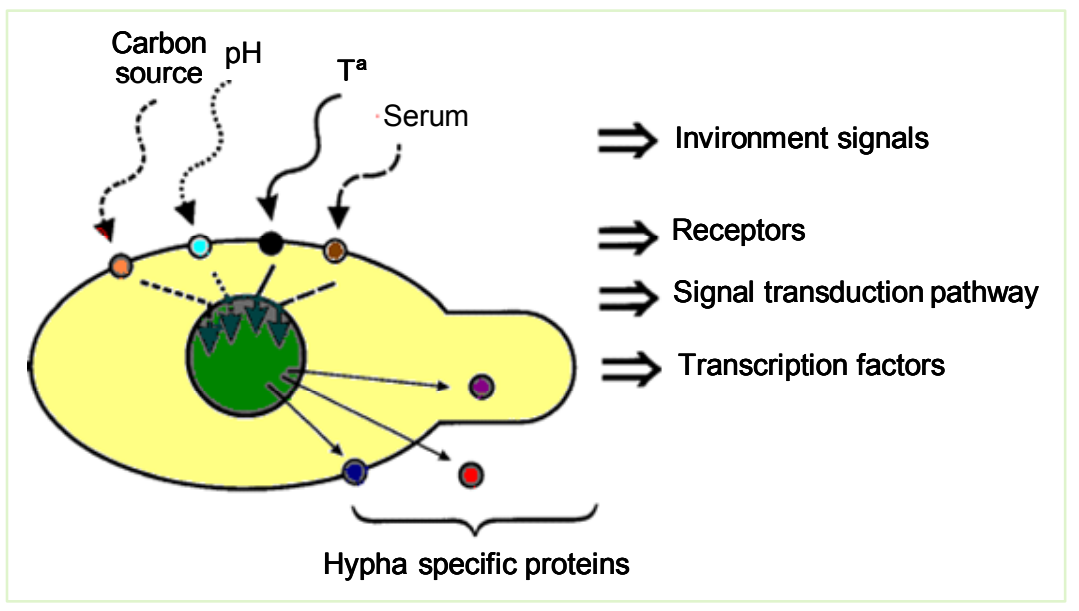

Figure 1.5 General scheme of hypha induction. Various environmental cues activate separate signalling pathways via sensor proteins. Signalling pathways may converge on separate or identical transcription factors and transcription factors may converge on common target genes to trigger expression of hyphaspecific genes.

The most powerful hypha-inducing protocol involves incubation of $C$. albicans at low cell densities in liquid medium containing serum, GlcNAc or other inducers, at $37^{\circ} \mathrm{C}$ (Davis-Hanna et al., 2008; Shepherd et al., 1980). Because of strong stimulation of several pathways, minor defects in signalling pathways are not detected under this condition. Liquid induction is usually monitored for a few hours, when initial germ tubes have developed into hypha that begin to bud off yeast cells. In contrast, on solid media cells develop into colonies over several days of growth, until (pseudo-) hyphal growth 
emerging from the colonies is recorded. The latter procedure is somewhat problematic, because (i) the molecular environment of a fully developed colony will differ greatly from the initial media composition, not allowing a clear definition of the inducing environment- in fact colonies 'condition' their environment by depletion of nutrients and by secretion of fungal compounds and (ii) cells may element efficiently during initial growth, but may revert quickly to yeast growth and the phenotype of a grown colony will be determined by not only the yeast-hypha transition, but also the reverse process. Ernst (Ernst, 2000a) found that the use of 1\% proline as a single carbon and nitrogen source in solid media strongly induced elements until microcolonies appeared, but growth later completely reverted to the yeast form, leading to a yeast-only appearance after several days.

\section{Serum and other inducers}

Serum of different sources is still the 'magic potion' to rapidly induce true hypha in $C$. albicans. The responsible factor is not albumin, since albumin-free serum from a rat mutant was as efficient at promoting hypha as normal serum (Feng et al., 1999). Two known inducers of hypha formation, $\mathrm{N}$-acetylglucosamine (GlcNAc) and proline may contribute to the serum effect since they are generated by degradation of serum (glyco-) proteins.

Thus, several independent signalling pathways are likely to be triggered by serum. Hypha arises within minutes at $37^{\circ} \mathrm{C}$ at $5-20 \%$ serum in liquid; on solid media hypha development is triggered within a few hours. Numerous other compounds induce hypha in vitro (Odds et al., 1988), but their significance for the infection process is unknown. The Efg1 protein is a strong regulator of morphogenetic processes in C. albicans, since it influences not only yeast-hypha inter-conversions (Lo et al., 1997; Noffz et al., 2008; Stoldt et al., 1997), but also regulates phenotypic switching and chlamydospore formation of this pathogen (Sonneborn et al., 1999a). The role of Efg1p in hyphal morphogenesis is fascinating, because under standard induction conditions, using serum or GlcNAc as inducers in liquid or on solid media, there is essentially a complete block of hyphal formation in strains lacking Efg1p (Noffz et al., 2008; Stoldt et al., 1997). On the other hand, under microaerophilic/embedded conditions hyphal formation is not defective at all in homozygous efg1 mutants, but rather appears stimulated (Sonneborn et al., 1999b). 


\section{Starvation}

In S. cerevisiae nitrogen limitation stimulates pseudohyphal growth (Gimeno et al., 1992). This morphogenesis is not detected in liquid medium, only on solid nitrogenlimiting medium (SLAHD); yeast colony first grows to a considerable size within several days and then lateral pseudohypha appear. C. albicans is also triggered on SLAHD medium to form filaments (true hypha) after several days' growth (Csank et al., 1998). A similar phenotype occurs on a medium containing mannitol as a source of carbon and a complex source of nitrogen (modified Lee's medium or Spider medium) (Liu et al., 1994). The late development of hypha on these media suggests that some media component has to be consumed before filamentation is triggered. It has been known for a long time that pre-growth of cells into stationary phase or a period of starvation enhances the efficiency of subsequent hyphal induction by inducers like serum or GlcNAc (Delbruck \& Ernst, 1993; Shepherd et al., 1980). The addition of ammonium salts to Spider medium did not block hyphal development, indicating that nitrogen limitation is not responsible for filamentation in this medium (Ernst, 2000a).

\section{Components of the cell signalling pathways}

Signal transduction networks permit cells to receive external stimuli and respond to the signals in an appropriate manner. Signal transduction describes a great number of biochemical events that transmit a signal from the cell exterior, through the cell membrane and through cytoplasm. This involves a number of molecules, including receptors, intermediate proteins and messengers. The signalling pathways are commonly used by an extensive array of biological ligands to modulate various cell processes, such as growth, differentiation and proliferation. These transduction pathways communicate information about the external environment to the inside of a cell. Signalling systems in fungi also regulate cell polarity, mating, pheromone control and hence they play a role in determining cell shape. Some of the known responses include changes in the cell cycle, polarized growth and modifications to the transcriptional profile of the cell.

Since the 50's, signalling pathways have been investigated by genetic and biochemical experimentation. In a large series of experiments, eukaryotic organisms were studied for their nutritional limitations and for their reactions to various environmental stresses, such as heat, oxidative, osmotic, or ethylic shock (Engebrecht, 2003). Pathogenic organisms sense and respond to the harsh conditions imposed by the host-activating components of signalling pathways that culminate in the expression of genes 
responsible for the virulence and differentiation of the pathogen. Therefore, studies on signal transduction in various fungi may reveal common conserved mechanisms of signal transduction as well as the differences between these organisms.

\section{- Mitogen-activated protein kinase cascades}

The Mitogen-Activated Protein Kinase (MAPK) signalling pathways (Nordle et al., 2007) play an important role in signal transduction in eukaryotic cells, where they modulate many cellular events including: mitogen-induced cell cycle progression through the G1 phase, regulation of embryonic development, cell movement and apoptosis, as well as cell and neuronal differentiation (Chen \& Thorner, 2007; Murray et al., 1998). These evolutionarily conserved pathways are organized in three-kinase modules consisting of a MAP kinase, an activator of MAP kinase (MAP Kinase Kinase or MEK) and a MAP Kinase Kinase Kinase (MEK Kinase, MEKK, or MAPK Kinase Kinase) (Arga et al., 2007; Dohlman \& Slessareva, 2006).

There are at least three distinct MAP kinase signal transduction pathways in mammalian cells, each named after the particular MAPK associated with it although in budding yeast $S$. cerevisiae has more than the three MAPK pathways (Levin-Salomon et al., 2009). MAP kinases are proline-directed serine/threonine kinases that are activated by dual phosphorylation in response to diverse extracellular stimuli. The dual phosphorylation occurs in the activation domain of MAPKs on the threonine and tyrosine residues in the sequence pTXpY (Adam et al., 2008; Alonso-Monge et al., 2003; Escote et al., 2004; O'Rourke et al., 2002). The dual phosphorylation is facilitated by dual-specificity MAP kinase kinases (MAPKKs or MEKs), which in turn are activated by serine/threonine phosphorylation by MAP kinase kinase kinases (MEKK) (Fig. 1.6).

\section{- The cAMP-activated protein kinase cascades}

The cAMP-PKA pathway plays a very important role in filamentation in S. cerevisiae, $C$. albicans and other fungi (Lee et al., 1975b; Lengeler et al., 2000). Nitrogen starvation in $S$. cerevisiae results in the formation of elongated buds termed pseudohypha, which is dependent on activation of the cAMP pathway (Gimeno et al., 1992; Kronstad et al., 1998; Lengeler et al., 2000). In C. albicans, an increase in cAMP levels accompanies the yeast-hypha transition, and inhibition of the cAMP phosphodiesterase induces this transition (Sabie \& Gadd, 1992). Previous reports of cAMP levels during the yeasthypha transition (Dhillon et al., 2003; Maidan et al., 2005; Maidan et al., 2008; Roman 
et al., 2007; Wolyniak \& Sundstrom, 2007) are difficult to compare because of the differences in strains and in experimental conditions. Nonetheless, it is clear is that the cAMP signal is less pronounced in C. albicans than in S. cerevisiae. Our understanding of the C. albicans cAMP-PKA pathway is based on considerable work with budding yeast.

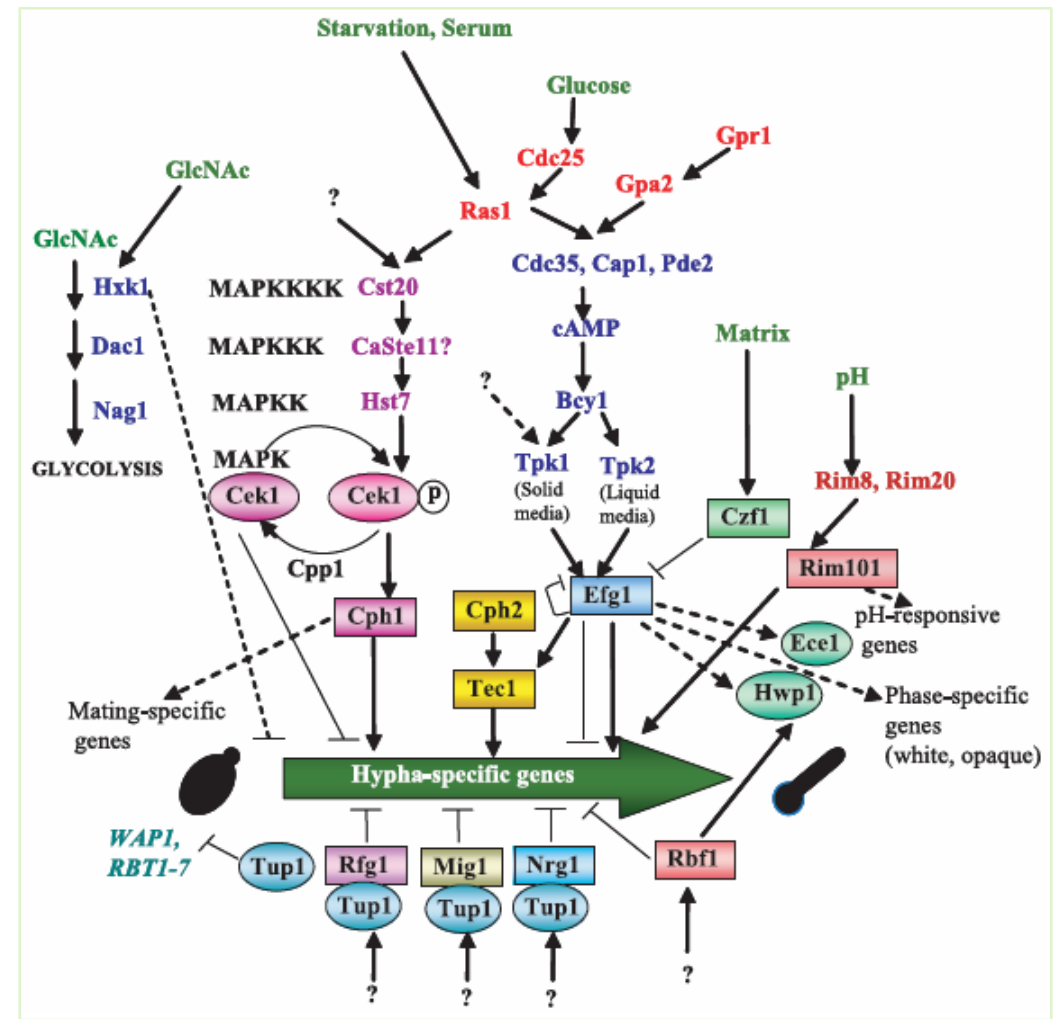

Figure 1.6 Regulation of dimorphism in C. albicans by multiple signalling pathways (Biswas et al., 2007)

\section{- pH induction pathway}

C. albicans causes infections in a broad range of host niches, which show significant differences in ambient $\mathrm{pH}$. For example, the mouse systemic $\mathrm{pH}$ is 7.3 , whereas the $\mathrm{pH}$ in the rat vagina is 4.5. The ability of Candida to react appropriately to (among other environmental variables) rather different $\mathrm{pH}$ environments is crucial for its pathogenicity. The environmental $\mathrm{pH}$ acts as a manipulator for many physiological functions, including morphogenesis (Fonzi, 2002). Under optimal $\left(37^{\circ} \mathrm{C}\right)$ temperature conditions, filamentation is favored by ambient $\mathrm{pH}$ values close to neutral and is considerably reduced at $\mathrm{pH}$ values lower than 6 . In contrast, the yeast form predominates almost exclusively at $\mathrm{pH} 4$ (Buffo et al., 1984). 


\section{- Negative regulation of filamentation}

Besides low $\mathrm{pH}$, low temperature and high cell density, it is known that high glucose concentrations down-regulate hyphal development of $C$. albicans in liquid media. Glucose repression of morphogenesis is also observed initially during growth on solid media, although glucose consumption permits filamentation after several days of growth. On solid media, high osmolarity also inhibits hypha formation (Alex et al., 1998). The presence of easily utilizable nitrogen sources, such as ammonium salts, modulates hyphal development negatively to some degree (Ernst, 2000a).

In the infected host, inhibition of filamentation by $\gamma$-interferon occurs at contact of $C$. albicans with lymphocytes (Kalo-Klein \& Witkin, 1990; Levitz \& North, 1996). Some chemical inhibitors of filamentation are known including diaminobutanone and some antifungals at low concentrations, such as amphotericin and azole antifungals; these compounds inhibit hyphal development by unknown mechanisms (Hawser, 1996; Hawser et al., 1996; Martinez et al., 1990). The Tup1 transcription factor may be involved in the hypha-repressed state in the presence of glucose and other noninducing conditions. In $S$. cerevisiae, Tup1 protein regulates about 60 genes involved in glucose regulation, oxygen stress response and DNA damage. A C. albicans homologue of Tup $1 p$ was identified that is $67 \%$ identical to S. cerevisiae Tup $1 p$ (Braun \& Johnson, 1997). Tup1p contains seven conserved WD40 repeats at the C-terminus, which could anchor Tup1p to some of its DNA-binding proteins and an N-terminal domain that could interact with a homologue of Ssn6p, as in S. cerevisiae (Keleher et al., 1992; Komachi \& Johnson, 1997). A homozygous C. albicans tup1 mutant grew in the filamentous form in all media tested; filaments on most of the media had the characteristics of pseudohypha, although in some of them had the appearance of true hypha. Pseudohypha of a tup1 mutant (unlike pseudohypha produced by EFG1 overexpression) (Braun \& Johnson, 1997; Stoldt et al., 1997) could not be induced to form germ tubes or true hypha by the addition of serum (Braun \& Johnson, 1997). Tup1p has other activities besides repression of mutants, because tup1 mutants failed to grow at $42^{\circ} \mathrm{C}$ grew faster on glycerol and had misshapen cell walls compared to the wildtype.

\section{Chromatin structure and nucleosomes}

In all living cells the DNA is wrapped around proteins called histones, thus forming chromatin. The repeating subunit of the chromatin, the nucleosome, consists of 146 DNA nucleotides wrapped around the histone core, which carries one subunit of each 
of the four histones: $\mathrm{H} 2 \mathrm{~A}, \mathrm{H} 2 \mathrm{~B}, \mathrm{H} 3$ and $\mathrm{H} 4$. The main function of the chromatin is to pack the DNA efficiently in the cell, but it was also shown to participate in crucial processes such as mitosis, replication, DNA damage and gene expression (Bakkenist \& Kastan, 2004)(Fig. 1.7).

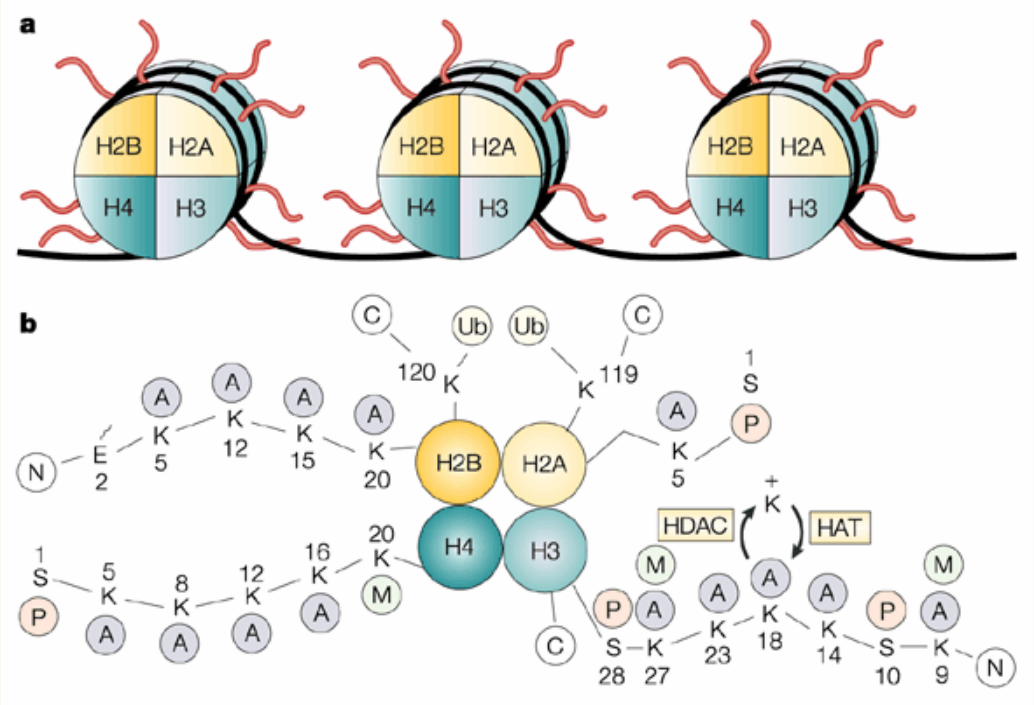

Figure 1.7 Nucleosome structure in eukaryotic cells

Much of the regulatory potential of the histones lies in their $\mathrm{NH}_{2}$ termini. The $\mathrm{NH}_{2}$ terminal tails, the first; 30 amino acids of each histone, are largely unstructured and contain high concentrations of lysine and arginine residues (Luger et al., 1997). The physical characteristics of the $\mathrm{NH}_{2}$-terminal tails are regulated by extensive posttranslational modifications (Verreault et al., 1998).

\section{Histone acetyltransferase complexes}

Changes in chromatin structure greatly influence all aspects of DNA metabolism. Remodeling of the chromatin structure at the nucleosomal level occurs by a variety of methods. One such remodeling is the direct covalent modification of histones, examples of which include acetylation, methylation, ubiquitination, and phosphorylation (Berger, 2007). A histone acetyltransferase (HAT) can be defined as an enzyme that acetylates core histones, which results in important regulatory effects on chromatin structure, assembly and gene transcription. The current understanding of HATs can be traced back over 40 years. In 1964, Allfrey and colleagues showed that histones can be modified by the addition of acetyl and methyl groups (Allfrey et al., 1964). Over the course of the next 15 years, a number of studies correlated the acetylation of histones with gene activity (Brownell \& Allis, 1996). Then, in 1979, Cano and Pestana isolated 
protein fractions with HAT activity from the larvae of brine shrimp (Cano \& Pestana, 1979). Another 15 or so years passed before the first HATs, Hat1p and Gcn5p, were isolated and cloned from S. cerevisiae (Brownell \& Allis, 1996; Kleff et al., 1995). Two years later, the first multi subunit nuclear HAT complex, SAGA (Spt-Ada-Gcn5acetyltransferase), was isolated (Grant et al., 1997). Over the past 10 years, the study of HATs has advanced significantly as they have become much more amenable to molecular and biochemical analysis. A number of HAT enzymes have been isolated from various organisms (Carrozza et al., 2003). The identification of new HATs has resulted in other important findings. It has been demonstrated that HATs are evolutionarily conserved from yeast to humans, that HATs generally contain multiple subunits (Kimura et al., 2005), and the functions of the catalytic subunit depend largely on the context of the other subunits in those complexes (Utley \& Cote, 2003). Furthermore, recent work on HAT complexes has resulted in their categorization on the basis of their catalytic domains. The picture is complicated by the observation that some HAT enzymes can modify different histone substrates and that some HAT enzymes also acetylate an ever growing number of non-histone substrates (Yang, 2004). In addition, histone acetylation is a dynamic reversible process. The balance of histone acetylation is important for proper cellular function and the cell has evolved enzymes that catalyse the removal of acetyl groups, termed histone deacetylases (HDACs) (Ekwall, 2005; Robyr et al., 2002). Histone acetylation is catalyzed by histone acetyltransferases (HATs), proteins that mediate the transfer of an acetyl moiety from acetyl CoA (acetyl-coenzyme A) to the $\varepsilon$-amino group of target lysine residues (Fig. 1.8).

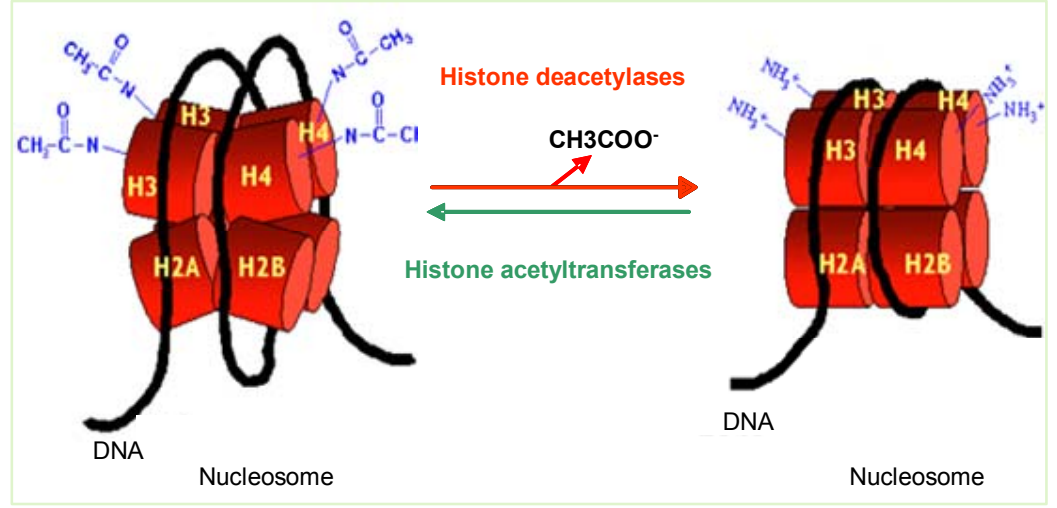

Figure 1.8 Histone acetyltransferases (HATs) remodeling. These enzymes add acetyl groups onto the basic amino acids in histone tails, which reduces the positive charge on the histone and weakens its binding to the DNA (which is negatively charged). This process can be reversed by histone deacetylases (HDACs), which remove the acetyl groups and cause the chromatin to recondense into the heterochromatin structure. 
HATs that acetylate nucleosomes are usually components of large multi-protein complexes, including NuA4, SAS-C and SAGA. The enzymatic removal of these acetyl groups from lysines is performed by histone deacetylases (HDACs) (Fig. 1.8). Histones can also be post-translationally modified prior to their deposition into chromatin (Benson et al., 2006).

\section{The histone acetyltransferase superfamily}

A large number of transcriptional regulators have been found to possess intrinsic HAT activity (Table 1.1) (Sterner \& Berger, 2000).

Table 1.1 HAT families and their transcription-related functions ACTR-activator of thyroid and RA receptor; HAT-histone acetyltransferase; MOF-male absent on first; MYST -MOZ, Ybf2/Sas3, Sas2, Tip60; PCAFp300/CBP associated factor; rOX-RNA on X; SRC-1- steroid receptor cofactor 1; not known (Lusser et al., 2001).

\begin{tabular}{|c|c|c|c|}
\hline HAT & HAT complex & Function & Organism \\
\hline \multicolumn{4}{|c|}{ Gcn5-related N-acetyltransferases (GNAT) } \\
\hline Gcn5 & Ada, SAGA & Coactivator of transcription & Ubiquitous \\
\hline Hat1 & Complex with Rbap48 & Cytoplasmic acetylation of $\mathrm{H} 4$ & Ubiquitous \\
\hline PCAF & PCAF complex & Coactivator of transcription & Mammals \\
\hline Hра2 & - & Unknown & Yeast \\
\hline Elp3 & RNA polymerase II complex & Transcription (elongation) & Yeast \\
\hline $\mathrm{CBP} / \mathrm{p} 300$ & $\begin{array}{l}\text { Associates with different } \\
\text { regulatory protein }\end{array}$ & Coactivator of transcription & Ubiquitous \\
\hline \multicolumn{4}{|c|}{ Nuclear receptor coactivators } \\
\hline ACTR & & Coactivator of transcription & Mammals \\
\hline SRC-1 & & Coactivator of transcription & Mammals \\
\hline TIF2 & & Coactivator of transcription & Mammals \\
\hline TAFII250 & TFIID & Factor associated with TBP & Ubiquitous \\
\hline \multicolumn{4}{|c|}{ MYST-family } \\
\hline Sas3 & Nun & Silencing & Yeast \\
\hline Esa1 & Nun & Cell cycle regulation & Yeast \\
\hline MOF & MSL complex & Gene dosage compensation & Insects \\
\hline MOZ & & Malignant diseases & Human \\
\hline Tip60 & Tip60 complex & HIV-Tat interaction & Human \\
\hline HBO1 & HBO complex & $\begin{array}{l}\text { Interacts with replication origin recognition } \\
\text { complex }\end{array}$ & Human \\
\hline
\end{tabular}

Sequence analysis of these proteins reveals that they fall into distinct families that show high similarity within families but poor to no sequence similarity between families (Kuo et al., 1998). Gcn5/PCAF family of HAT proteins (GNAT family) function as coactivators for a subset of transcriptional activators. This family depended on target site is classified as histone acetyltransferase A and histone acetyltransferase B. 
GCN5 (General Control of Non-derepressed 5) was the first discovered protein capable of acetylating nuclear histones. GCN5 was initially identified by Penn et al (Penn et al., 1983 ) in a yeast mutation screen identifying genes involved in amino acid biosynthesis. Mutations were induced by UV-light irradiation and clones unable to grow in media deficient in preformed amino acids were considered to have disruptions in the regulation of their amino acid biosynthesis pathways. The screen lead to the discovery of 5 genes involved in the derepression (induction) and expression of proteins involved in the synthesis of histidine, arginine, tryptophan and methionine (Penn et al., 1983). The genes were named GCN1 through GCN5.

Eukaryotic GCN5 acetyltransferases influence diverse biological processes by acetylating histones and non-histone proteins and by regulating chromatin and genespecific transcription as part of multiprotein complexes. In lower eukaryotes and invertebrates, these complexes include the yeast ADA complex that is still incompletely understood; the SAGA (Spt-Ada-Gcn5 acetylase) complexes from yeast to Drosophila that are mostly co-activators; and the ATAC (Ada Two-A containing) complex, only known in Drosophila and also still poorly characterized.

In contrast, vertebrate organisms, express two paralogous GCN5-like acetyltransferases (GCN5 and PCAF), which have been found so far only in SAGAtype complexes referred to here after as the STAGA (SPT3-TAF9-GCN5/PCAF acetylase) complexes (Wang et al., 2008 ). It has been reported that in budding yeast Gcn5p is involved in cell cycle progression, whereas its absence induces several mitotic defects, including inefficient nuclear division, chromosome loss, delayed G2 progression, and spindle elongation. The fidelity of chromosome segregation is finely regulated by the close interplay between the centromere and the kinetochore, a protein complex hierarchically assembled in the centromeric DNA region (Vernarecci et al., 2008).

\section{Histone acetyltransferase B (HAT1 and HAT2)}

In $S$. cerevisiae Hat1p, together with Hat2p and Hif1p, forms the histone acetiltransferase B complex. The functional significance of the association of Hif1p with the Hat1p/Hat2p complex is confirmed by the observation that hif1 and hat 1 strains display similar defects in telomeric silencing and DNA double strand break repair. Functional analysis revealed that Hif1p is a novel histone chaperone that selectively interacts with histones $\mathrm{H} 3$ and $\mathrm{H} 4$. Hif1p is also a chromatin assembly factor, 
promoting the deposition of histones in the presence of a yeast cytosolic extract. In vivo, the nuclear Hat $1 \mathrm{p} / \mathrm{Hat} 2 \mathrm{p} / \mathrm{Hif} 1 \mathrm{p}$ complex is bound to acetylated histone $\mathrm{H} 4$, as well as histone H3. In eukaryotes Hat1p, has been linked to histone deposition, nucleosome formation and chromatin assembly during ongoing DNA replication and DNA repair, rather than to a more common function in the regulation of transcription (Ai \& Parthun, 2004; Verreault et al., 1998). Hat1p was originally identified as a cytoplasmic enzyme that acetylates free, but not chromatin-bound, histone H4 (Kleff et al., 1995; Parthun et al., 1996). Hat2p, a conserved and evolutionary protein, homolog of the human retinoblastoma binding protein Rba48p, acts as adaptor for high affinity binding of Hat1p to histone H4 (Kleff et al., 1995; Parthun et al., 1996), whereas Hif1p is a histone chaperone as mentioned above (Ai \& Parthun, 2004).

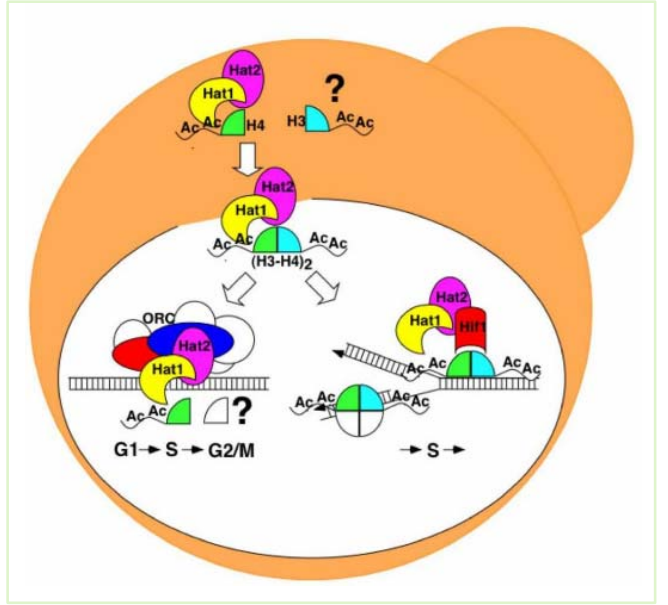

Figure 1.9 The current view on the different associations of Hat1p within the cell. In addition to the cytoplasmic Hat1p/Hat2p complex, the Hat1p/Hat2p-Hif1p chromatin assembly complex is recruited to chromatin during S-phase.

Hif1p is detected exclusively in nuclei and may accompany newly modified H3-H4 tetramers to facilitate incorporation into nascent chromatin during DNA synthesis (Suter et al., 2007). The association of Hif1p with acetylated $\mathrm{H} 4$ requires for Hat1p and Hat2p to provide a direct link between type $B$ histone acetyltransferases and chromatin assembly. In addition, the histone $\mathrm{H} 4$ associated with the nuclear Hat1p/Hat2p/Hif1p complex contains novel posttranslational modifications in the core domain. One site of core domain acetylation, lysine 91 , lies at the interface between the $\mathrm{H} 3 / \mathrm{H} 4$ tetramer and $\mathrm{H} 2 \mathrm{~A} / \mathrm{H} 2 \mathrm{~B}$ dimers, and might be critical for the chromatin assembly (Ai \& Parthun, 2004). In eukaryotes synthetic N-terminal histone H4 peptides found that whereas the HAT-B complex acetylates only Lys12, recombinant Hat1p is able to modify Lys 12 and Lys5. Both Lys12 and Lys5 of soluble, non-chromatin-bound histone $\mathrm{H} 4$ are in vivo targets of acetylation for the yeast HAT-B enzyme. Moreover, co-immunoprecipitation 
assays revealed that Lys12/Lys5-acetylated histone $\mathrm{H} 4$ is bound to the HAT-B complex in the soluble cell fraction. Both Hat1p and Hat2p, but not Hif1p, are required for the Lys12/Lys5-specific acetylation and for histone H4 binding. HAT-B-dependent acetylation of histone $\mathrm{H} 4$ was detected in the soluble fraction of cells at distinct cell cycle stages, and increased when cells accumulated excess histones. Strikingly, histone $\mathrm{H} 3$ was not found in any of the immunoprecipitates obtained with the different components of the HAT-B enzyme, indicating the possibility that histone $\mathrm{H} 3$ is not together with histone H4 in this complex (Poveda \& Sendra, 2008).

Finally, the exchange of Lys for Arg at position 12 of histone $\mathrm{H} 4$ did not interfere with histone $\mathrm{H} 4$ association with the complex, but prevented acetylation on Lys 5 by the HAT-B enzyme, in vivo as well as in vitro (Makowski et al., 2001; Poveda \& Sendra, 2008). Yeast strains deficient in either HAT1 or HAT2 exhibit no obvious growth deficiencies (Kleff et al., 1995). However, gene silencing at telomeres is reduced when hat 1 or hat 2 mutant null alleles are combined with substitution mutations blocking acetylation of the amino terminal tail of histone H3 (Kelly et al., 2000b). Moreover, hat1 mutant in combination with $\mathrm{N}$-terminal lysine substitution alleles in histone $\mathrm{H} 3$ confers hypersensitivity to DNA-damaging agents (Qin \& Parthun, 2002). Similar phenotypes are recapitulated with deletions in the HIF1 gene in combination with $\mathrm{H} 3$ tail mutants (Ai \& Parthun, 2004). Collectively, these data point to the overlapping of acetylation of the N-terminal tail of histone $\mathrm{H} 3$ and Hat1p-mediated acetylation of histone $\mathrm{H} 4$ in chromosome dynamics.

In C. albicans, like other eukaryotes, HAT1 and HAT2 are conserved in other fungal species and that the most homologous regions are within the HAT and WD40 domain (Fig. 1.10 and 1.11). Many yeast genes encode histone acetytransferase proteins in $C$. albicans and their relative phenotype is totally unknown. Thus, our labotaroty have performed a phenotypic analysis of yeast strain carrying deletions of HAT1, HAT2 and other genes encoding members of the HATs and HDACs family of $C$. albicans.

A. CaHat1p (413 aa)

Hat:1_til

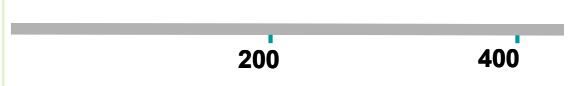

Hati_il

Histone acetyl transferase HAT1 N-terminus (20 -170 aa)

MOZ/SAS family (124 - 308 aa)

Acetyltransferase (GNAT) family (196 - 246 aa)

B. CaHat2p (486 aa)

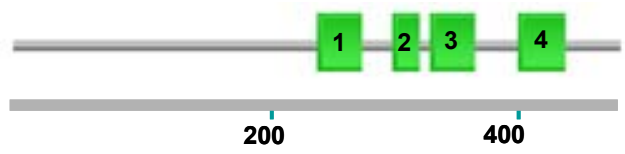

WD40

1. $245-283$ aa

2. 304 - 329 aa

3. 334 - 373 aa

4. 405 - 444 aa 
The C. albicans genome contains two ORFs that potentially encode histone acetyltransferase $B$ complex. A search of the NCBI database (http://www.ncbi.nlm.nih.gov/BLAST/) indicates that a Candida albicans protein named Hat1 (orf19.705) shares the highest similarity with Pichia guilliermondii (62\%), Pichia stipitis (69\%), Lodderomyces elongisporus (69\%), Debaryomyces hansenii (61\%), Saccharomyces cerevisiae (57\%), Kluyveromyces lactis (44\%), Candida glabrata (44\%), Yarrowia lipolytica (37\%), Schizosaccharomyces pombe (33\%) and Homo sapiens (26\%). The GeneBank accession number for the C. albicans HAT1 and HAT2 nucleotide sequence are NW_139542 and NC_007436 respectively.
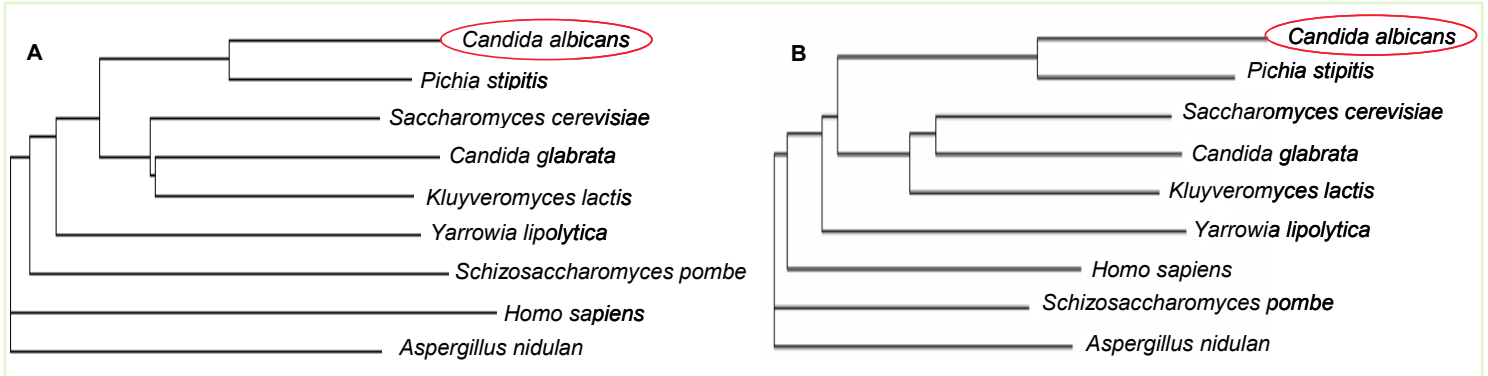

Figure 1.11 Dendograms representing the phylogenetic relationships of $C$. albicans HAT1 (A) and HAT2 (B)

\section{Are type B histone acetyltransferases cytoplasmic or nuclear?}

Cytoplasmic localization was one of the original defining characteristics of type $B$ histone acetyltransferases. But are these enzymes really cytoplasmic? The idea that type B histone acetyltransferases are located in the cytoplasm arose from the fact that histone acetyltransferase activities, usually specific for free histone $\mathrm{H} 4$, are found in the soluble fraction following lysis of a variety of eukaryotic cell types. However, the fact that a protein does not pellet with nuclei, does not necessarily mean that it was not nuclear before cell lysis as many proteins have been shown to leak out of isolated nuclei (Decker et al., 1987; Li \& Kelly, 1984). The identification of Hat1p as a type B histone acetyltransferase provided the tools to more definitively determine the subcellular localization of this enzyme. In $S$. cerevisiae, evidence from a variety of different experiments suggests that the enzyme is actually both cytoplasmic and nuclear (Fig. 1.12). 


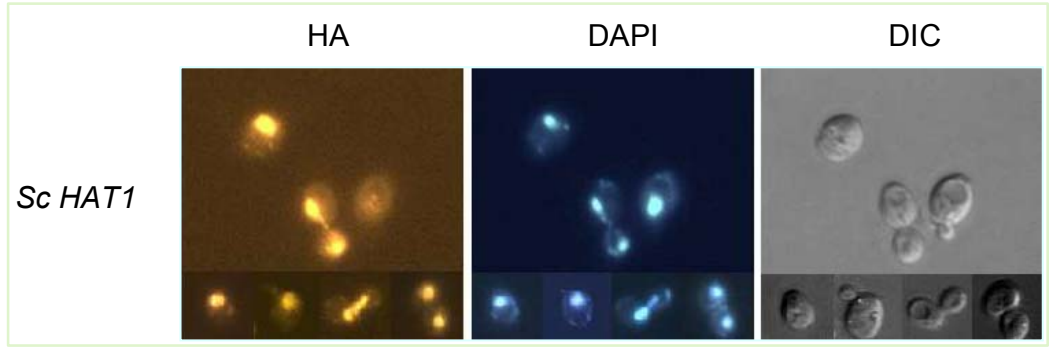

Figure 1.12 The subcellular localization of Hat1p in S. cerevisiae (Poveda et al., 2004)

Deletion of the gene encoding HAT1 not only eliminates the H4-specific histone acetyltransferase present in cytoplasmic extracts, but also causes the loss of an activity present in nuclear extracts as well (Parthun et al., 1996; Ruiz-Garcia et al., 1998). Also, distinct Hat1p/Hat2p-containing complexes can be purified from both cytoplasmic and nuclear extracts (Ai \& Parthun, 2004; Poveda et al., 2004). Localization of native Hat2p shows a strong concentration in the nucleus with a significant staining throughout the cytoplasm as well (Ai \& Parthun, 2004) (Fig. 1.13).

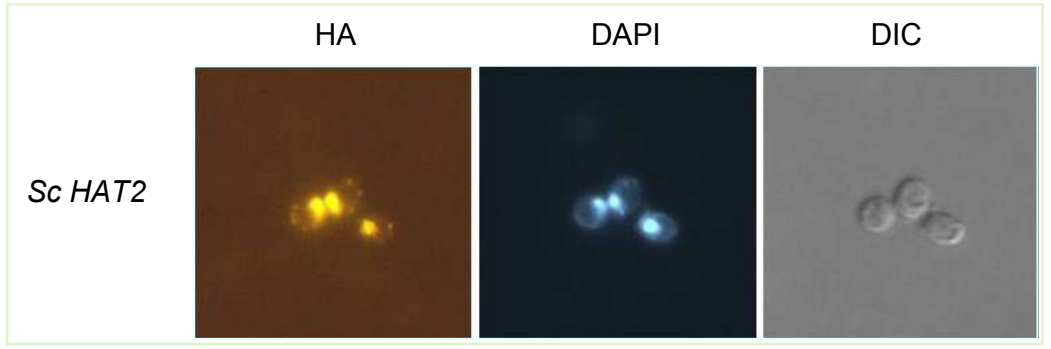

Figure 1.13 The subcellular localization of Hat2p in S. cerevisiae (Poveda et al., 2004)

Localization of epitope-tagged Hat1p and Hat2p (expressed from their native promoters) shows a similar pattern with dense staining in nuclei and diffuse staining in the cytoplasm.

\section{Histone deacetylase complexes}

Histone deacetylases (HDACs) reverse chromatin acetylation and promote transcriptional repression and silencing. Dozens of proteins have been found to possess intrinsic HDAC activity (Cress \& Seto, 2000; Grozinger \& Schreiber, 2002). In S. cerevisiae histone deacetylases comprise a family of 10 genes, which are grouped into classes I-III based on their homology to their respective yeast orthologues. Class I, consist of 3 family members (ScRpd3p, ScHos1p and ScHos2p), Class II consist of 2 family members (ScHda1p and ScHos3p) and class III include of ScSir2p, ScHst1p, ScHst2p, ScHst3p and ScHst4p of family members. In the human genome histone 
deacetylases comprise a family of 18 genes, which are grouped into classes I-IV based on their homology to their respective yeast orthologues. Classes I, II and IV consist of 11 family members, which are referred to as "classical" HDACs, whereas the 7 class III members are called sirtuins. The phylogenetic grouping of HDACs clearly shows that, in general yeast have fewer HDACs than humans. Because redundancy is often a problem in genetic analysis, Saccharomyces cerevisiae and Schizosaccharomyces pombe are excellent model systems for the study of HDACs.

\section{The Sin3-histone deacetylase (HDAC) corepressor}

Mammals possess two highly related $\operatorname{Sin} 3$ proteins, $m \operatorname{Sin} 3 \mathrm{~A}$ and $\mathrm{mSin} 3 \mathrm{~B}$, which serve as scaffolds tethering HDAC enzymatic activity, and numerous sequence-specific transcription factors to enable local chromatin regulation at specific gene targets (David et al., 2008). SIN3 was first identified genetically as a global regulator of transcription. $\operatorname{Sin} 3 p$ is a large protein composed mainly of protein-interaction domains, whose function is to provide structural support for a heterogeneous Sin3/histone deacetylase (HDAC) complex. In addition to HDACs, Sin3p can sequester other enzymatic functions, including nucleosome remodeling, DNA methylation, $\mathrm{N}$-acetylglucosamine transferase activity and histone methylation. Since the Sin3/HDAC complex lacks any DNA-binding activity, it must be targeted to gene promoters by interacting with DNAbinding proteins. Although most research on $\operatorname{Sin} 3 p$ has focused on its role as a corepressor, mounting evidence suggests that $\operatorname{Sin} 3 p$ can also positively regulate transcription. Furthermore, $\operatorname{Sin} 3 p$ is a key to the propagation of epigenetically silenced domains and is required for centromere function. Thus, it provides a platform to deliver multiple combinations modifications to the chromatin, using both sequence-specific and sequence-independent mechanisms (Silverstein \& Ekwall, 2005). In C. albicans Sin3p, a component of a specific histone deacetylase complex, is shown to bind to the EFG1 promoter. The $\sin 3$ mutants grew as budding pseudohypha and are unable to form true hypha, similar to strains constitutively expressing EFG1. It has been reported that the PKA signalling pathway, in addition to its importance in the initial steps of filament formation, is part of a feedback loop that controls EFG1 expression allowing continued hypha formation in inducing conditions. This auto-regulation of EFG1 expression is probably mediated through the Sin $3 p$-containing histone deacetylation complex (Tebarth et al., 2003). The $\operatorname{Sin} 3 p$ is optimized for multiple protein interactions with its four-paired amphipathic helices and a histone interaction domain (HID) (Fig. 1.14). 


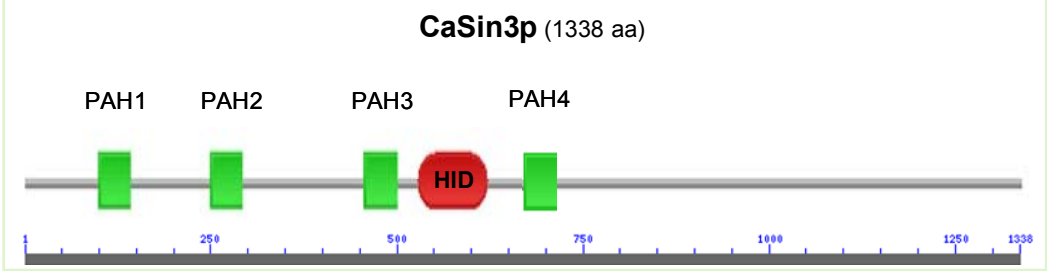

Figure1.14 Estructure of Sin3p in C. albicans contains four paired amphipathic helix (PAH) and HID domain.

The core of this complex, which is highly conserved from yeast to man, consists of eight components in mammals: SIN3, HDAC1, HDAC2, RbAp46, RbAp48, SAP30, SAP18 and SDS3. The yeast complexes contain only one HDAC each and seem to lack the SAP proteins. The resulting complexes provide specialized combinations of enzymatic specificities to the target chromatin. Sin3p has no intrinsic DNA-binding activity. Yet it must localize to a gene promoter if it is to exert any control over its transcriptional regulation. The targeting of the Sin3/HDAC complex is accomplished by flexibly interacting with DNA-binding proteins.

$\operatorname{Sin} 3 p$ is able to directly interact with transcription factors, although it sometimes requires an adaptor molecule. Co-repressors such as N-CoR and SMRT provide such a link between Sin3p and the nuclear hormone receptors. By regulating the interaction between $\sin 3 p$ and its targeting proteins, the cell is able to tightly regulate gene expression. It also clearly has a role in silencing at both centromeres and the rDNA, as well as DNA-methylation-linked silencing. Other transcription-independent roles for Sin $3 p$ include influencing retrotransposon transposition frequency, replication timing and DNA repair. By providing a flexible scaffold for various chromatin modifying enzymes and DNA-binding proteins, $\operatorname{Sin} 3$ is able to globally regulate gene expression and to help maintain overall genome stability.

\section{The cDNA microarray analysis of differential gene expression in C. albicans}

Microarray technology evolved from Southern blotting, where fragmented DNA is attached to a substrate and then probed with a known gene or fragment. The use of a collection of distinct DNAs in arrays for expression profiling was first described in 1987 , and the arrayed DNAs were used to identify genes whose expression is modulated by interferon (Kulesh et al., 1987). These early gene arrays were made by spotting cDNAs onto filter paper with a pin-spotting device. The use of miniaturized microarrays for gene expression profiling was first reported in 1995 (Schena et al., 1995), and a complete eukaryotic genome (S. cerevisiae) on a microarray were published in 1997 
(Lashkari et al., 1997). Genomic research tools such as microarrays have provied to be important resources to study the complex regulation of genes that respond to environmental perturbations (Shaw et al., 2007). It consists of an arrayed series of thousands of microscopic spots of DNA oliogonucleotides, called features, each containing picomoles of a specific DNA sequence. This can be a short section of a gene or other DNA element that are used as probes to hybridize a cDNA or cRNA sample (called target) under high-stringency conditions. Probe-target hybridization is usually detected and quantified by fluorescence-based detection of fluorophore-labeled targets to determine relative abundance of nucleic acid sequences in the target. The recently developed DNA microarray technology provides a powerful and efficient tool to rapidly compare the differential expression of a large number of genes. The near completion of sequencing the $C$. albicans genome has made it possible to employ genomic technologies, such as microarray analysis, to aid in identifying key genes involved in such clinical problems as the acquisition of high-level resistance to azole antifungal agents. In addition, DNA microarrays provide a snapshot of an organism's genome in action by revealing the relative transcript levels of thousands of genes at a time (Liu et al., 2005). Microarray analysis gives researchers the ability to identify genes involved in processes such as biofilm formation and acquisition of azole resistance and to use the data in a way that may lead to clinical approaches to inactivate these genes and improve patient outcomes. In human by cDNA microarray technology and methodology more focuses on their application in molecular classification of tumors, drug sensitivity and resistance studies and identification of biological markers of cancer. Differences in gene expression underlie many of the phenotypic variations in $C$. albicans, yet approaches to characterize such differences on a genome-wide scale are well developed by using cDNA microarray techniques. Phenotypic diversity can often be traced to the differential expression of specific regulatory genes (Carroll, 2000; Gompel et al., 2005). 


\begin{abstract}
Analysis/publication
DNA microarray based on arrayed-primer extension technique for identification of pathogenic fungi responsible for invasive and superficial mycoses (Campa et al., 2008)
\end{abstract}

Genome-wide expression profiling of the response to terbinafine in Candida albicans using a cDNA microarray analysis (Zeng et al., 2007)

cDNA microarray analysis of differential gene expression and regulation in clinically drug-resistant isolates of Candida albicans from bone marrow transplanted patients $(X u$ et al., 2006)

cDNA microarray analysis of differential gene expression in Candida albicans biofilm exposed to farnesol (Cao et al., 2005)

Transcriptional and physiological adaptation to defective protein-Omannosylation in Candida albicans (Cantero et al., 2007)

\section{Abstract of result}

The microarray was tested for its specificity with a panel of reference and blinded clinical isolates. The APEX technique was proven to be highly discriminative, leading to unequivocal identification of each species, including the highly related ones $C$. parapsilosis, C. orthopsilosis and C. metapsilosis. Because of the satisfactory basic performance traits obtained, such as reproducibility, specificity, and unambiguous interpretation of the results, this new system represents a reliable method of potential use in clinical laboratories for parallel one-shot detection and identification of the most common pathogenic fungi.

In this work A total of 222 genes were found to be responsive to terbinafine, including 121 up-regulated genes and 101 down-regulated genes. These included genes encoding membrane transport proteins belonging to the members of the ATPbinding cassette (ABC) or major facilitator superfamily (MFS; CDR1, AGP2, GAP6, PHO84, HOL3, FCY23, VCX1), genes involved in stress response and detoxification (CDR1, AGP2, HOL3), and gene involved in the ergosterol biosynthesis pathway (ERG12). The up-regulation of the gene encoding the multidrug resistance efflux pump CDR1 may contribute to the terbinafine resistance in Candida albicans.

In this work more than 198 differentially expressed genes were identified and they were confirmed and validated by RT-PCR independently. Not surprisingly, the resistant phenotype is associated with increased expression of CDR mRNA, as well as some common genes involved in drug resistance such as CalFU5, CaRTA2 and CalFD6. Meanwhile, some special functional groups of genes, including ATP binding cassette (ABC) transporter genes (IPF7530, CaYOR1 and CaPXA1), oxidative stress response genes (CaALD5, CaGRP1, CaSOD2 and IPF10565), copper transport and iron mobilization-related genes (CaCRD1/2, CaCTR1/2, CaCCC2 and CaFET3) were found to be differentially expressed in the resistant isolates. Furthermore, among these differentially expressed genes, some co-regulated with CaCDR1, CaCDR2 and CalFU5, such as CaPDR16 and CalFD6, have a DRE-like element and may interact with TAC1 in the promoter region. These findings may shed light on mechanisms of azole resistance in $C$. albicans and clinical antifungal therapy.

A total of 274 genes were identified as responsive, with 104 genes up-regulated and 170 genes down-regulated. Independent reverse transcription-PCR analysis was used to confirm the important changes detected by microarray analysis. In addition to hyphal formation-associated genes (e.g., TUP1, CRK1 and PDE2), a number of other genes with roles related to drug resistance (e.g., FCR1 and PDR16), cell wall maintenance (e.g., CHT2 and CHT3), and iron transport (e.g., FTR2) were responsive, as were several genes encoding heat shock proteins (e.g., HSP70, HSP90, HSP104, CaMSI3 and SSA2). Further study of these differentially regulated genes is warranted to evaluate how they may be involved in $C$. albicans biofilm formation. Consistent with the down-regulation of the cell surface hydrophobicityassociated gene (CSH1), the water-hydrocarbon two-phase assay showed a decrease in cell surface hydrophobicity in the farnesol-treated group compared to that in the control group. Our data provide new insight into the molecular mechanism of farnesol against $C$. albicans biofilm formation.

Comparisons of genome-wide transcript patterns of each pmt mutant revealed commonly down-regulated genes involved in glycolysis and glycerol production. Increased phosphorylation of the Cek1p- but not the Mkc1p-MAP kinase, as well as increased transcript levels for some stress-related genes was detected in the pmt 1 strain but not in the other pmt mutants. The transcriptomal pattern after short-term inhibition of Pmt1p activity confirmed stress responses, but did not indicate an alteration of glycolytic flow. Short- but not long-term adaptation to Pmt1p inhibition required signalling components Cek1p, Mkc1p, Efg1p and Tpk1p. Cna1p (calcineurin) but not its downstream effectors Crz1p and Crz2p was generally essential to allow growth during Pmt1p inhibition; accordingly, cyclosporin A strongly inhibited growth of the pmt1 mutant. The lack of Pmt isoforms influenced transcript levels for the remaining isoforms both positively and negatively, suggesting complex cross-regulation among PMT genes. These results confirm individual functions of Pmt isoforms but suggest a common biphasic adaptation response to Pmt deficiency. While known signalling pathways modulate adaptation for a short-term, long-term adaptation requires calcineurin, adjustments of remaining Pmt activities and of glycolytic flow. 



\section{Abstract}

We have studied the behavior of a $C$. albicans which encode two histone acetyltransferases. We have deleted the C. albicans HAT1 gene in a homozygous hat $2 \Delta$ mutant genetic background and generated a hat $1 \Delta$, hat $2 \Delta$ double mutant strain. The double mutant behaves as the hat $1 \Delta$ simple mutant, respect to growth rate, hyphal outgrowth, chlamydospore formation and sensitivity to Calcofluor white, SDS, Congo red, Amphotericin B, Fluconazole, Itraconazole and Caffeine. In a location analysis by GFP-fusion to Hat1p and Hat2p, we have found that Hat1p and Hat2p are mainly localized in the nucleus and that location of Hat $2 p$ is dependent on the Hat $1 p$.

We were also observed that the double mutant like hat1 $\Delta$ simple mutant showed attenuated virulence in a mice model. We have carried out the transcriptional profile of the hat $2 \Delta$ and hat $1 \Delta$, hat $2 \Delta$ during both yeast mode of growth and during the yeasthypha transition. We have also compared our results with those obtained with the $C$. albicans hat $1 \Delta$ simple mutant. All the three mutants show a different transcriptional profile supporting the idea that HAT1 and HAT2 control different set of genes and that both acetyltransferases interact to regulate the expression of another set of $C$. albicans genes.

\section{Introduction}

In the eukaryotic organisms, acetylation of histone is a reversible process that depends on two different sets of enzymatic activities, histone acetyltransferases (HAT) and histone deacetylases (HDAC). This post-translational modification is involved in processes such as activation or repression of gene transcription, nucleosome assembly during replication, DNA repair and recombination or cell cycle and growth control (Carrozza et al., 2003; Kurdistani \& Grunstein, 2003; Rosaleny et al., 2005). The yeast HAT1 was the first HAT gene described (Kleff et al., 1995) that specifically modifies Lys 12 of free histone $\mathrm{H} 4$ and has been implicated in the acetylation of cytoplasmic histone molecules required for post-replicative nucleosome assembly (Poveda \& Sendra, 2008; Rosaleny et al., 2005). In Saccharomyces cerevisiae, it has been demonstrated that HAT-B complex is mainly localized in the nucleus and that is composed of three proteins, Hat1p, Hat2p and Hif1p (Ai \& Parthun, 2004; Parthun, 2007; Poveda et al., 2004). Deletion of HAT1, in combination with specific histone H3 amino terminal tail mutations, results in a significant defect in telomeric silencing (Kelly 
et al., 2000b). Hat2p acts as a bridge between Hat1 and Hif1 proteins (Poveda et al., 2004) and is required for high affinity binding of Hat1p to histone H4 (Parthun et al. 1996). It seems to be essential for all the functions carried out by Hat1p, because deletion of HAT2 always produces similar defects to those of HAT1 (Kelly et al., 2000b; Parthun et al., 1996; Ruiz-Garcia et al., 1998). Deletion of HIF1 displays similar defects to those described in telomeric silencing (Ai \& Parthun, 2004; Poveda et al., 2004) and in DNA double-strand break repair to those described for the deletion of HAT1 Parthun, 2004). At present, in C. albicans no unique phenotype has been specifically and directly associated to a particular HAT-B subunit. We wanted to investigate whether deletion of HAT1 and HAT2 play any role in the yeast-hypha transition or yeast mode of growth and virulence. We have observed that hat1 $\Delta$ and hat2 $\Delta$ mutants display a different phenotype and transcriptional profile. The hat $1 \Delta$, hat $2 \Delta$ double mutant also display a different transcriptional profile of both simple mutants. All these results indicate that Hat $1 p$ and Hat2 $p$ have different functions and that very few overlaps exits between them.

\section{Experimental procedures}

\section{Strains, media and culture conditions}

The S. cerevisiae strains used were pJ69-4A and pJ69-4 $\alpha$, bearing the yeast two-hybrid system. The relevant genotypes of the strains used are listed in Table 2.1. For general purposes, yeasts were grown in 250-ml flasks containing $50 \mathrm{ml}$ yeast peptone dextrose (1\% Yeast extract, 2\% Bactopeptone, 1\% Glucose) or YNB $(0.67 \%$ Yeast Nitrogen Base without aminoacids (YNB w/o aa, Difco), 1\% Glucose) plus the required amino acids for plasmid maintenance. For the induction of hypha in C. albicans, $4 \%(\mathrm{v} / \mathrm{v})$ bovine calf serum (GIBCO) was added to the culture media. The Lee, Spider and SLAHD media were prepared as described (Buffo et al., 1984; Castilla et al., 1998; Lee et al., 1975a; Liu et al., 1994). Yeast growth temperature was $28^{\circ} \mathrm{C}$ for general purposes and $37^{\circ} \mathrm{C}$ for expression of the phenotype in the mutant strains or induction of the filamentous morphology. 
Table 2.1 Strains and plasmids used in this study

\begin{tabular}{|c|c|c|c|}
\hline & Relevant genotype or characteristics & $\begin{array}{c}\text { Parental } \\
\text { strain }\end{array}$ & Reference \\
\hline \multicolumn{4}{|l|}{ Strains } \\
\hline SC5314 & Clinical isolated & - & (Gillum, 1984) \\
\hline CAI4 & ura34::imm434/ura34::imm434 & CAF2-1 & (Fonzi and Irwin, 1993) \\
\hline CAMR & ura34::imm434/URA3 & CAI4 & (Rodrigues and Dominguez, 2005) \\
\hline $\mathrm{RDH} 4$ & $\begin{array}{l}\text { hat2 } 2 \text { ::hisG /hat2 } 2:: \text { hisG } \\
\text { ura34:: imm434/ura34:: imm434 }\end{array}$ & CAI4 & (Degano and Dominguez, 2005) \\
\hline RGL4 & $\begin{array}{l}\text { hat14::hisG /hat14::hisG } \\
\text { ura34:: imm434/ura34:: imm434 }\end{array}$ & CAI4 & (Degano and Dominguez, 2005) \\
\hline MAH1 & $\begin{array}{l}\text { hat1 } 1: \text { hisGURA3hisG/HAT1 } \\
\text { ura3 }:: \text { imm } 434 / \text { ura3 } \triangle:: \text { imm } 434 \\
\text { hat } 2 \Delta:: \text { hisG /hat } 2 \Delta:: \text { his }\end{array}$ & $\mathrm{RDH} 4$ & present work \\
\hline MAH2 & hat14::hisG/HAT1 & MAH1 & present work \\
\hline MAH3 & $\begin{array}{l}\text { hat1 } 1: \text { hisG/hat1 } 1: \text { hisGURA3hisG } \\
\text { ura3 }:: \text { imm } 434 / \text { ura3 } \triangle:: \text { imm } 434 \\
\text { hat2 }:: \text { hisG /hat } 2 \Delta:: \text { hisG }\end{array}$ & MAH2 & present work \\
\hline $\mathrm{MAH} 4$ & 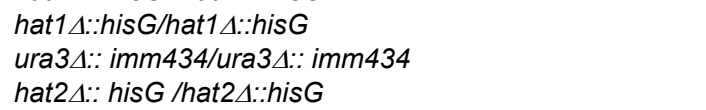 & MAH3 & present work \\
\hline MAHR1 & 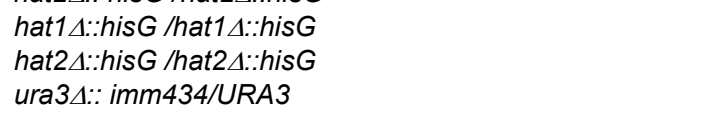 & MAH4 & present work \\
\hline RDHR9 & $\begin{array}{l}\text { hat2 } 2 \text { ::hisG /hat } 2 \Delta:: \text { hisG } \\
\text { ura3 }:: \text { imm434/URA3 }\end{array}$ & $\mathrm{RDH} 4$ & present work \\
\hline RGLR6 & $\begin{array}{l}\text { ura3A::imm434/URA3 } \\
\text { hat14::hisG /hat14::hisG }\end{array}$ & RGL4 & present work \\
\hline PJ69-4A & $\begin{array}{l}\text { MATa trp1-901ura3-52 his3-200 } \\
\text { leu2-3,112 gal4 } \text { gal80 } \text { GAL2-ADE2 } \\
\text { LYS2::GAL1-HIS·met2::GAL7-lacZ }\end{array}$ & & (James et al., 1996) \\
\hline \multicolumn{4}{|l|}{ Plasmids } \\
\hline pAG1 & $\begin{array}{l}\text { Plasmid containing the ampicillin resistance gene, the } \\
C \text {. albicans } A C T 1 \text { promotor fused to the GFP protein } \\
\text { (with Smal/BamHI places), the } L E U 2 \text { and URA3 genes } \\
\text { and the ARS2 sequence. }\end{array}$ & pIR4 & (Novo et al., 2004) \\
\hline pGEM-T & $\begin{array}{l}\text { Plasmid containing ampicillin resistance gene, T7 and } \\
\text { SP6 RNA Polymerase transcription initiation site, T7 } \\
\text { and SP6 RNA Polymerase promoter, multiple cloning } \\
\text { site, lacZ start codon, lac operon sequences, lac } \\
\text { operator, } \beta \text {-lactamase coding region, phage f1 region, } \\
\text { binding site of pUC/M13 Forward Sequencing Primer, } \\
\text { binding site of pUC/M13 Reverse Sequencing Primer. }\end{array}$ & & Promega (Robles, 1994) \\
\hline pMR1 & $\begin{array}{l}\text { Plasmid containing the reinsertion cassette of the } \\
\text { URA3 gene. }\end{array}$ & & (Rodriguez and Dominguez, 2005) \\
\hline pZRG1 & $\begin{array}{l}\text { Plasmid containing the HAT2 gene under control of the } \\
\text { ACT1 promotor fused in frame to the GFP protein (in } \\
\text { the Smal site). }\end{array}$ & & present work \\
\hline pAHR1 & $\begin{array}{l}\text { Plasmid containing the HAT1 gene under control of the } \\
\text { ACT1 promotor fused in frame to the GFP protein (in } \\
\text { the Smal site). }\end{array}$ & & present work \\
\hline pGBD-C & $\begin{array}{l}\text { Plasmid containing the } S \text {. cerevisiae ADH1 promotor, } \\
\text { GAL4 DNA-binding domain polypeptide, TRP1 coding } \\
\text { sequence, Ampicillin resistance gene. }\end{array}$ & & (James et al., 1996) \\
\hline pROS1 & $\begin{array}{l}\text { Plasmid containing the } S \text {. cerevisiae ADH1 promotor, } \\
\text { GAL4 DNA-binding domain polypeptide, TRP1 coding } \\
\text { sequence, Ampicillin resistance gene, HAT2 gene. }\end{array}$ & & present work \\
\hline pGAD-C & $\begin{array}{l}\text { Plasmid containing the promoter fragment carrying the } \\
\text { truncated } S \text {. cerevisiae } A D H 1 \text { promotor, GAL4 } \\
\text { activation domain polypeptide, } L E U 2 \text { coding sequence, } \\
\text { Ampicillin resistance gene. }\end{array}$ & & (James et al., 1996) \\
\hline pMAH1 & $\begin{array}{l}\text { Plasmid containing the promoter fragment carrying the } \\
\text { truncated } S \text {. cerevisiae } A D H 1 \text { promotor, GAL4 } \\
\text { activation domain polypeptide, } L E U 2 \text { coding sequence, } \\
\text { ampicillin resistance gene, HAT2 gene. }\end{array}$ & & present work \\
\hline pJBR3 & $\begin{array}{l}\text { Hat1 promoter and terminator flanked in URA3-marked } \\
\text { pSNC1 vector }\end{array}$ & & (Degano and Dominguez, 2000) \\
\hline
\end{tabular}


Plasmid pJBR3 was constructed in our laboratory (Degano, Ph.D thesis in process). Gene disruption was performed by the "Ura-blaster" protocol (Fonzi and Irwin, 1993). C. albicans CAI4 genomic DNA was used as template for PCR using the oligonucleotides (Table 2.2). A 5.1-kb Notl-Xhol fragment was isolated and used to transform strain RDH4 (hat2A). Correct integration of the cassette into the HAT1 locus of the URA3 ${ }^{-}$ transformants was verified by PCR and Southern blot analysis. Spontaneous URA3 derivatives of one of the heterozygous disruptants were selected on medium containing 5-fluoroorotic acid (Sigma). These clones were screened by PCR and Southern blot hybridization to identify those which had lost the URA3 gene via intrachromosomal recombination mediated by the hisG repeats. The procedure was then repeated to delete the remaining functional allele of HAT1.

\section{Southern hybridization}

Samples of genomic DNA $(10 \mu \mathrm{g})$ were digested with restriction enzymes and the resulting fragments were separated by electrophoresis in 1\% agarose gel in TAE buffer (2 M Tris-HCl/NaOAc, $50 \mathrm{mM}$ EDTA pH 8.0). The agarose gel was then submerged in $0.25 \mathrm{~N} \mathrm{HCl}$ for $15 \mathrm{~min}$ (twice), in $0.5 \mathrm{M} \mathrm{NaOH} / 1.5 \mathrm{M} \mathrm{NaCl}$ for $30 \mathrm{~min}$, and finally in 0.5 $\mathrm{M}$ Tris- $\mathrm{HCl}, \mathrm{pH} 7.5 / 1.5 \mathrm{M} \mathrm{NaCl}$ for a further $30 \mathrm{~min}$. The DNA was then transferred onto a Hybond- $\mathrm{N}^{\mathrm{TM}}$ membrane (Amersham Pharmacia Biotech) by capillarity, and the membrane was blocked by UV cross-linked 120 mJoules $\mathrm{cm}^{-2}$ for $40 \mathrm{~s}$ (Statagene UV crosslinker) to ensure DNA immobilization. Prehybridization was performed in $1 \%$ $\mathrm{NaCl}, 1 \%$ Dextran sodium sulphate salt (Amersham) and 1\% SDS with denatured salmon sperm DNA (Clontech) for $4 \mathrm{~h}$ at $65^{\circ} \mathrm{C}$. The blot was then hybridized with ${ }^{32} \mathrm{P}-$ labelled DNA probe $\left(\alpha^{32} \mathrm{P}\right.$, dCTP with specific activity $\left.>3000 \mathrm{Ci} / \mathrm{mmol}\right)$, with the same solution for at least $16 \mathrm{~h}$ at $65^{\circ} \mathrm{C}$. The membrane was then washed in SSC $2 \times$ (twice) for $5 \mathrm{~min}$ at room temperature and in SSC $1 \times, 0.1 \%$ SDS for 45 minutes at $65^{\circ} \mathrm{C}$. Detection of the hybridized probe was carried out according to the manufacturer's instructions for the ${ }^{32} \mathrm{P}$ labelling protocol and detected with a BAS-1500 Storage Phosphor Imageing System (Fujifilm).

\section{DNA manipulations}

Except where specified, standard procedures were used for DNA manipulation (Sambrook, 2001). C. albicans and S. cerevisiae transformations were carried out using the protoplast and lithium acetate methods respectively (Kohler et al., 1997; 
Rose, 1990). Genomic DNA from CAI4 C. albicans and mutant strains were obtained as described elsewhere (Sherman, 1986). The plasmid bearing GFP3 and optimized for the genetic code of $\boldsymbol{C}$. albicans has been described in (Barelle et al., 2004; Cormack et al., 1997) and plasmids bearing the cassette for GFP3 tagging directly to the chromosome have been described in (Gerami-Nejad et al., 2001).

\section{The yeast two-hybrid system}

The yeast two-hybrid system was carried out as have been described by James et al (James et al., 1996). To construct the plasmids for the two-hybrid system, the complete HAT1 and HAT2 genes were isolated from $C$. albicans genomic DNA using two primer sets: "CaHAT1F and CaHAT1R" and "CaHAT2F and CaHAT2R," respectively (Table 2.2). The corresponding fragment was digested and cloned into the pGAD-C and pGBD-C expression vector at the Smal/Sall site. Both plasmids were used to transform the S. cerevisiae pJ69-4A and pJ69-4 $\alpha$ strains. Transformants were selected on SD minus Leu and Trip media in the presence of plasmid. The transformants that display the expected phenotypes was conjugated. The resultant transformants contain pGAD$\mathrm{C}$ and pGBD-C was tested for GAL4 activity and survival on selective media: SD/-Leu/Trip/-His/-Ade.

Table 2.2 Oligonucleotides used in this study

\begin{tabular}{|c|c|c|c|}
\hline Primer & Sequence $5^{\prime}-3^{\prime}$ & Primer & Sequence $5^{\prime}-3^{\prime}$ \\
\hline $\begin{array}{l}\text { URA1 } \\
\text { URA2 } \\
\text { AMH1 } \\
\text { IP2 } \\
\text { IP3 } \\
\text { MAH38 } \\
\text { MAH39 } \\
\text { MAH40 } \\
\text { MAH41 } \\
\text { MAH32 } \\
\text { MAH33 } \\
\text { RD48 } \\
\text { RD49 }\end{array}$ & $\begin{array}{l}\text { GGATACTATCAAACAAGAGG } \\
\text { AATGCTGGTTGGAATGCTTA } \\
\text { CCGAACATCAACCAATCGT } \\
\text { TTACAATCAAAGGTGGTCC } \\
\text { GGTACAGTTGTTCCTCACA } \\
\text { GGTTGTTAATGCACCAAGAGA } \\
\text { CTCTTGTTCTCCAAACTGC } \\
\text { ACCAATCCTGAAGCAGCAC } \\
\text { CAAAGCTTGATAAGCAGTCTG } \\
\text { GGCAATCACATCAAGACT } \\
\text { TGTTCAACTTCAAGTCTC } \\
\text { GTATAGTACTGCAGTTGGCT } \\
\text { CCAACTCGAGCAACCCTC }\end{array}$ & $\begin{array}{l}\text { HAT2GFPR } \\
\text { HAT1GFPF } \\
\text { HAT1GFPR } \\
\text { CaHAT2F } \\
\text { CaHAT2R } \\
\text { HAT1GFPCO } \\
\text { CaHAT1F } \\
\text { CaHAT1R } \\
\text { HAT1 inner } \\
\text { HAT1 outer } \\
\text { HAT2 iner } \\
\text { HAT2 outer }\end{array}$ & $\begin{array}{l}\text { CCCGGGTGGTGGATCTTTCATTTTTGTATCCTC } \\
\text { CCCGGGATGTCATCCGCAAAAGAA } \\
\text { CCCGGGTGGTGGCACTTTTTGCTTTTGGAA } \\
\text { CCCGGGATGTTTTCGAGACCATTAGAG } \\
\text { GTCGACATCTTTCATTTTTGTATCCTC } \\
\text { CCCGGGTGGTGGAATGTTCAACTTCAAGTCT } \\
\text { CCCGGGATGTCATCCGCAAAAGAACAA } \\
\text { GTCGACTTACACTTTTTGCTTTTTGGA } \\
\text { TTCCTTTATTGAATCGACCCA } \\
\text { TCCAGGTATTTTATACCCATT } \\
\text { ACTTCCATTAGGTGAAACTTT } \\
\text { ATAACTACGAATAACACCATC }\end{array}$ \\
\hline
\end{tabular}

RLM-RACE

The C. albicans transcript was mapped using the FirstChoice RLM-RACE kit (Ambion). RNA was isolated from CAI4 exponential cultures by breaking cells in a microdismembrator (Braun, Melsungen), following by trizol extraction method as described in Galar Fungal standard operating procedures for RNA extraction. Total RNA was treated with Calf Intestinal alkaline Phosphatase (CIP) to remove the $5^{\prime}$-phosphate from non-full-length uncapped RNA. The 5' cap was removed from full-length mRNA 
by treatment with Tobacco Acid Pyrophosphatase (TAP), leaving a 5'-monophosphate to which a $5^{\prime}$ RACE adapter $(0.3 \mu \mathrm{g} / \mathrm{ml})$ oligonucleotide (5'GCUGAUGGCGAUGAAUGAACACUGCGUUUGCUGGCUUUGAUGAAA3') was ligated using T4 RNA ligase. Random-primed reverse transcription and nested PCR were used to amplify the $5^{\prime}$ transcript. The outer PCR reaction used the 5' RACE outer primers, whereas the inner PCR reaction used the 5' RACE inner primers (Table 2.2). The 5' RACE inner PCR products were cloned into pGEM-T (Promega) and sequenced.

\section{DNA sequencing and sequence analysis}

Both strands of the PCR product described above were sequenced using the DNA sequenciation service from University of Salamanca with an Applied Biosystems ABI PRISM 3100 Genetic Analyzer. For sequence analysis DNAstar program was used.

\section{$\mathrm{NaCl}$, Calcofluor white, Caffeine, SDS, Fluconazole, Itraconazole, Amphotericin B} and Hygromycin sensitivities

Methods for testing the $C$. albicans strains were similar for all the effectors. Cultures were grown in $50 \mathrm{ml}$ of YEPD medium until the exponential phase and diluted to an O.D $600_{\mathrm{nm}}$ of 0.4 . Five microliters of pure and $1 / 10$ serial dilutions of each cell culture were spotted onto YEPD plates containing $\mathrm{NaCl}(1 \mathrm{M})$, Calcofluor white $(1 \mathrm{mg} / \mathrm{ml})$, Caffeine $(15 \mathrm{mM})$, SDS $(0.05 \%)$, Fluconazole $(2 \mu \mathrm{g} / \mathrm{ml})$, Itranconazole $(4 \mu \mathrm{g} / \mathrm{ml})$, Amphptericin B $(100 \mu \mathrm{g} / \mathrm{ml})$, Hygromycin $(300 \mu \mathrm{g} / \mathrm{ml})$ and Congo red $(0.5 \mathrm{mg} / \mathrm{ml})$. Differences in growth were recorded after incubation of the plates at $28^{\circ} \mathrm{C}$ for 4 days.

\section{Zymolyase sensitivity phenotypic test}

Cultures of the CAI4, hat $1 \Delta$, hat $2 \Delta$ simples and double mutant, were grown in YEPD medium until the exponential phase. Cells were washed twice in water and resuspended in $10 \mathrm{mM}$ Tris- $\mathrm{HCl}\left(\mathrm{pH} \mathrm{7.5)}\right.$ ) and $0.3 \% \beta$-mercaptoethanol. $2 \times 10^{7}$ cells were resuspended in the same buffer containing Zymolyase 20T at a concentration of $0.01 \mathrm{mg} / \mathrm{ml}$. The optical density at $600_{\mathrm{nm}}$ was measured at the start of the incubation and every $20 \mathrm{~min}$ thereafter. The decreased in optical density reflected the portion of cells that have been lysed.

\section{Alcian blue binding assay}


The Alcian blue binding assay was carried out using the method of Herrero et al (Herrero et al., 2002). A series of solutions containing different amounts of Alcian blue was prepared in $0.02 \mathrm{~N} \mathrm{HCl}$ and the optical density at $600_{\mathrm{nm}}$ of each solution was determined. A standard curve was plotted of the O.D values versus amounts of Alcian blue. To quantify Alcian blue binding to the cell surface, $1000 \mu \mathrm{l}$ exponential-phase yeast cells (O.D $600_{\mathrm{nm}}, 2.5$ ) was centrifuged, and the cells were washed twice with $1 \mathrm{ml}$ of $0.02 \mathrm{~N} \mathrm{HCl}$ and resuspended in $1 \mathrm{ml} 0.02 \mathrm{~N} \mathrm{HCl}$ containing $100 \mu \mathrm{g}$ Alcian blue. The cell suspension was allowed to stand for $10 \mathrm{~min}$ at room temperature and then centrifuged for $3 \mathrm{~min}$ to pellet the cells. The O.D $600_{\mathrm{nm}}$ of the supernatant was measured. The amount of dye bound to the cells was calculated by subtracting the amount of dye in the supernatant from $100 \mu \mathrm{g}$.

\section{Mouse strain and infection model}

Pathogen-free 4-week-old CD1 mice were purchased from Animal Science Center of Salamanca University. For inoculation, fresh cultures of C. albicans were washed, resuspended in ice-cold PBS and counted with a hemocytometer. Cell concentration was adjusted with PBS to $5 \times 10^{8}$ yeast cells per $200 \mu$ l. Microscopic examination showed the cell suspension to be predominantly composed of single cells, with minimal clumping. Yeast suspensions were placed on ice until ready for injection. Immediately prior to injection, the yeast suspensions were agitated for 10 to 15 seconds and loaded into 1-ml syringes fitted with a $30 \mathrm{G}$ needle. Two hundred microliters of suspension was introduced into peritoneum, delivering a total of $5 \times 10^{8}$ yeast cells. Mice were returned to their cages and monitored for 30 days.

\section{Green fluorescent protein fusions to CaHAT1 and CaHAT2}

GFP3 was placed at the carboxyl terminus of CaHAT1 and CaHAT2. Two prolines were inserted between both proteins in order to preserve their correct folding. The pAG1 plasmid (a kind gift of Gonzalez-Novo) (Gonzalez-Novo et al., 2004) is based on the pIR4 plasmid, which carries the ACT1 promoter and a C. albicans ARS sequence. The CaHAT1 and CaHAT2 ORF were amplified by PCR and cloned in the PAG1 plasmid bearing the GFP and releasing the stop codon. These plasmids thus bore the CaHAT1-GFP and CaHAT2-GFP fusion under control of the ACT1 promoter. This construction was introduced into $C$. albicans CAl4 and hat $1 \Delta$ and hat $2 \Delta$ mutant strains.

\section{Microscopy}


The observation of GFP-fusion proteins was performed. Images were captured with a Leica DMRXA (Leica, Germany) fluorescence microscope equipped with a $63 \times$ objective and a Leica DFC 350 FX monochrome digital camera, using the Qfish 2.3 program. Images were processed with Adobe Photoshop.

The cell and colony morphology on liquid medium and plates were inspected microscopically using a Leica and Zeiss Stemi SV 6 stereoscopic microscope, respectively.

\section{RNA isolation, cDNA preparation and microarray hybridization}

C. albicans CAI4 (HAT1, HAT2), RDH4 (hat2 $\Delta$ ) and MAH4 (hat1 $\triangle$, hat2 $\Delta$ ) strains were grown in YNB and Lee cultures for yeast mode of growth and during the yeast-hypha transition, respectively. Cells were harvested at room temperature, resuspended in a small volume of the supernatant and immediately frozen by releasing small drops of cell samples into liquid nitrogen. Cells were then stored at $-80^{\circ} \mathrm{C}$ till RNA extraction. Total RNA was isolated by breaking cells in a microdismembrator (Braun, Melsungen, Germany), followed by trizol extraction method as described in Galar Fungal standard $\begin{array}{llll}\text { operating for } & \text { focedures extraction }\end{array}$ (http://www.pasteur.fr/recherche/unites/Galar_Fungail/), keeping the cells frozen in liquid nitrogen at all times. RNA was checked for integrity by electrophoresis and quantified by spectrophotometric (Hitachi, U-2001) analysis at $260_{n m}$. The C. albicans microarrays used in this study were manufactured by Eurogentec SA (Ivoz-Ramet, Belgium) in collaboration with the European Galar Fungail Consortium. Two independent sets of RNA from CAI4 (control) and mutant cells (biological replicates) were used in these studies to prepare two independent cDNA probe sets. Fifteen micrograms of total RNA sample were added to a mixture of $1 \mu \mathrm{l}$ of oligo (dT1218)(Sigma), $1 \mu \mathrm{l}$ primer mix (0.1 pmole/ $\mu \mathrm{l}+1 \mu \mathrm{g} / \mu \mathrm{l}$ AncT) (C. albicans-specific primer mix Plus) (Eurogentec); $3.6 \mathrm{mM}$ of each dATP, dGTP, dTTP; 1 mM dCTP (Sigma); 1 mM Cy3- or Cy5-dCTP (Amersham); and 0.1 M DTT (Invitrogen). The reaction mixture was denatured at $65^{\circ} \mathrm{C}$ for $5 \mathrm{~min}$, incubated at $42^{\circ} \mathrm{C}$ for $5 \mathrm{~min}$, after which $1 \mu \mathrm{l}$ of RNAsin (Promega, Madison, WI, USA) and $200 \mathrm{U}$ of Superscript II reverse transcriptase (Invitrogen) were added to the mixture. The reaction proceeded at $42^{\circ} \mathrm{C}$ for $1 \mathrm{~h}$ after which an additional $200 \mathrm{U}$ of Superscript II reverse transcriptase was added, and the reaction mixture was incubated at $42^{\circ} \mathrm{C}$ for an additional hour. To stop the reaction, EDTA ( $\mathrm{pH} \mathrm{8.0)} \mathrm{and} \mathrm{sodium} \mathrm{hydroxide} \mathrm{were} \mathrm{added} \mathrm{to} \mathrm{a} \mathrm{final} \mathrm{concentration} \mathrm{of} 5 \mathrm{mM}$ and $0.4 \mathrm{M}$, respectively, and the mixture was incubated at $65^{\circ} \mathrm{C}$ for $20 \mathrm{~min}$. Finally, acetic acid was added to achieve a final concentration of $0.4 \mathrm{M}$. The labelled cDNA probes 
were purified using Qia-Quick columns (Qiagen, Valencia, CA, and USA) following the manufacturer's instructions. The cDNA probes were then fluorescently labelled. One set of cDNA probes was labelled using Cy5 for those representing RNA from mutant's cells and Cy 3 for those representing RNA from control's cells. The second set was labelled using Cy3 for those representing RNA from mutant's cells and Cy5 for those representing RNA from control's cells. Five microlitres each of the Cy3- and Cy5labelled probes were mixed with $50 \mathrm{mg}$ of heat denatured salmon sperm DNA (Sigma, Aldrich), incubated at $95^{\circ} \mathrm{C}$ for 2 min and snap-cooled on ice. The mixture was added to $60 \mu \mathrm{l}$ of hybridization buffer (DIG easy hyb; Roche, Basel, Switzerland) and applied to the array slides under cover slips (Sigma). Hybridization was performed at $42^{\circ} \mathrm{C}$ overnight in a humidified chamber (Corning Life Sciences, Acton, MA, USA). After that microarrays were washed fallowing Eurogentec recommendations and dried by centrifugation at $1100 \mathrm{rpm}$ at room temperature. Slides were scanned using a GenePix 4000B scanner.

\section{Data analysis}

GenePix 4.0 software (Axon Instruments) was used for image analysis and data visualization. Preliminary normalization, logarithmic transformation and determination of correlation coefficients were performed with Excel 2003 (Microsoft). Data were loaded into GeneSpring version 5.0.3 (Silicon Genetics, CA, and USA). Signals were log-transformed and per chip normalized by an intensity-dependent method (Lowess) applied to the print-tip region. Significance analysis of the results was conducted using Student's $t$ test (Gene-Spring). Data with $\rho$ values of $\leq 0.02$ were considered to be significant. In the present study, only spots with a mean balanced differential expression ratio $\geq 1.5$ or $\leq 0.66(1 / 1.5)$ were considered to be differentially expressed for both spots representing a given cDNA on the array in two independent experiments.

\section{Results}

\section{Determination of transcription initiation site using RNA ligase-mediated Rapid Amplification of $5^{\prime}$ cDNA Ends (5' RLM-RACE) of HAT1 and HAT2 genes}

To identify transcription initiation sites, RLM-RACE was performed. The results are shown in Fig. 2.1. We have amplified one band of $\sim 450 \mathrm{bp}$ and $\sim 500$ bp for HAT1 and HAT2 respectively (Fig 2.1A) that was cloned in pGEM-T. After sequencing four different clones, three putative transcription start sites (two identical and two different) were identified for HAT1. These products may have originated from different 
transcription start sites. The three positions 21,24 and 48 bp can be taken as the transcription start site (Fig. 2.1B). In any case the HAT1 transcript is relatively short only a few bp upstream of the ATG codon. We must point out that very few data about the length of transcripts exist in C. albicans.

We have detected three putative transcription start sites for HAT2, all of them inside of coding sequence (ORF) that has been predicted by "in silico" analysis (CandidaDB) (Fig. 2.1C). Our result open the possibility that the real place of start of the CaHAT2 gene is the one marked in red in Fig. 2.1C and purification of the protein is necessary to assess this point.

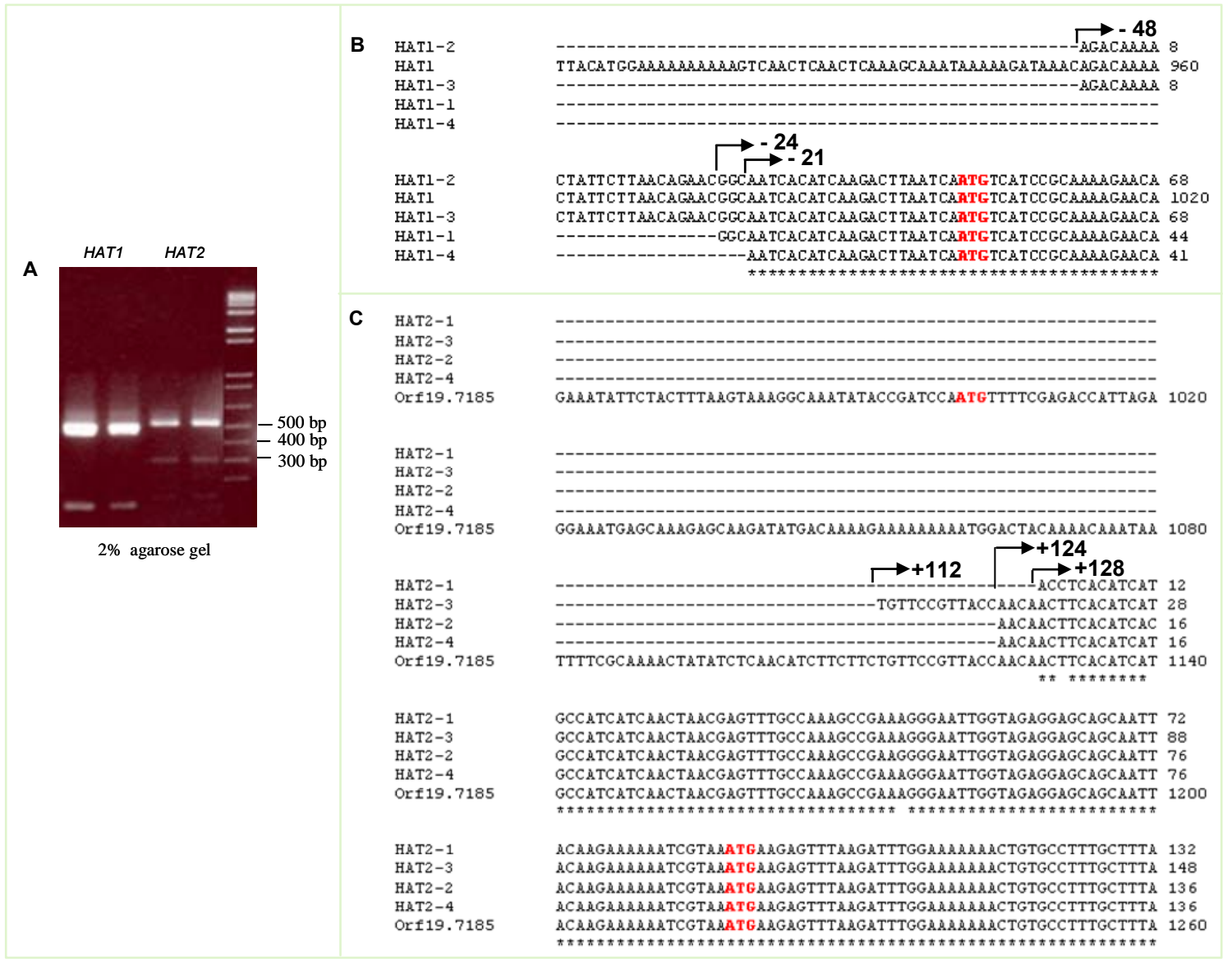

Figure 2.1 Mapping of HAT1 and HAT2 transcrirtion initiation sites by RLM-RACE. Predicted of the transcription start site of HAT1 and HAT2 genes 
C. dubliniensis

C. albicans

C. tropicales

P. stipitis

C. dubliniensis

C. albicans

C. tropicales

$P$. stipitis

C. dubliniensis

C. albicans

C. tropicales

P. stipitis

C. dubliniensis

C. albicans

C. tropicales

P. stipitis

c. dubliniensis

C. albicans

C. tropicales

P. stipitis

C. dubliniensis

C. albicans

C. tropicales

P. stipitis

C. dubliniensis

C. albicans

C. tropicales

P. stipitis

C. dubliniensis

C. albicans

c. tropicales

P. stipitis

C. dubliniensis

C. albicans

C. tropicales

P. stipitis

MPSTNEFAKAERE 14 MFSRPLEEMSKEQDMTKEKKMDYKTNNFRKTISQHLLSFRYQQLHIMPSSTNEFAKAERE 60 - . MTITQKDLAVAERE 14 *. : : : $* * \star * *$

LVEEQQLQEKIVNEEFKIWKKTVPLLYDFIHTFALDSPSLVFQWLPTTNVS - -QSDLELK 72 LVEEQQLQEKIVNEEFKIWKKTVPLLYDFIHTFALDNPSLVFQWLPTTSVS - -QSDLELK 118 LANEQQLQEKIVNEEFKIWKKTVPLLYDFVHTFALDSPSLVFQWLPDYNVS - - QSDLEVK 72 IVEEHQLKEKVVNEEFKIWKKTVPLLYDTIHTYVLDYPSLAIKWLPDYTYSDNKNSVNVK 74

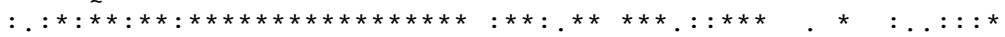

FLIGTNTINKADNYLKLASINLPSTLVG - - - - - ATESIPVPSDDID - TSNFKVITQWK 124 FLIGTNAINKSENYLKLTSISLPSTLVG - - - - - ATDSIPVPSDGID - TSNFKVVTQWK 170 FLIGTNTINKSENYLKLGSVTLPSTLVENS - - - TNPQGIPIPTEDLD - TSNFRIINQWK 127 FLIGTNTSHNSSNYLKLGSVNIPSTLAPDFSTVNPDVDSITVPSSVIEDTSDFRILSKWK 134

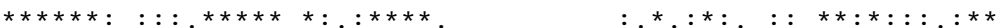

QSQEVNKLKVSPNGSLAVGFNADGVLRSYNLDNFDSVDYKYHKQGGIALDWVDNNGFLSG 184 QTQEINKLKVSPNGSLAVGFSADGVIRSYNLDNFDSVDYKYHKQGGIALDWVDNNGFLSG 230 QSCEINKLKVSSNGGLAVGFGADGIIRGFNLKNYDIVDYKYHKQEGSALNWINENSFISG 187 QTSEINKLDISPNGKKVLSFNSDGVVHSYDLENNDVIDYKYHKSEGYALTWFGNDSFISG 194

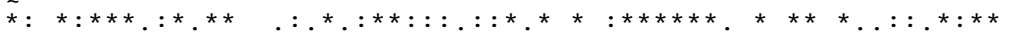

SNDSQIALWQVDKPSTPLQLFKGHHGAINDISY -VKEKHLFGSVSDDSTTQFHDSRVNSA 243 SNDAQIALWQVDKSSTPLQLFKGHHGAINDISS - IKEKHLFGSVSDDSTTQFHDTRVNAT 289 AKDSOIALWOVDKPSTPIOLFKGHRGAINDLSS - IKGKTLFGSVSDDSTTOFYDGRIGSI 246 SNDSQIALWSLDKPSTPIQLFKSHNGAVNDISYNPNFVSIFGSVSDDSSTQFHDSRASGD 254 $::^{*}:{ }^{* * * *} .:^{* *},{ }^{* * *}:{ }^{* * *} \cdot{ }^{*},{ }^{* *}:{ }^{* *}:{ }^{*} \quad: \quad:{ }^{* * * * * * *}:{ }^{* * *}:{ }^{*}{ }^{*} \ldots$

DINPVITVENSHIQKCIQFHPDIPTLYATGGKDNVVSLYDMRNYSTPFRKFYGHNDSVRQ 303 DINPVITVENSHIQNCIQFHPDIQTLYATGGKDNVVSLYDIRNYSTPFRKFYGHNDSVRQ 349 DANPVISVENNHIQNCIQFHPDIHTMYATAGKDNIVSLYDMRNYKTPFRKFYGHNDTIRQ 306 - - NPVIKQENQHIQMAISVHPEIETLYATGGKDNVVSLYDIRNYKIPLRKFFGHNDSVAG 312

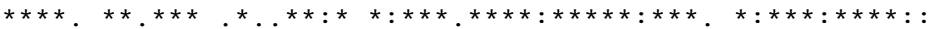

LQWDWNNPDILVSCGLDKRIIFWDLKNLDEDFTYPDAMSNGKDTNSKKKQAVKTDPCLKY 363 LQWDWNNPDILVSCGLDKRIIFWDLKNLDEDFTYPDATSNGKDTNSKRKQAVKTDPCLKY 409 LQWDSYNPNLLVSCGLDKRVLFWNLESLDEDYTYPDQTSNGKDSNSKKKQVNKVDPCLKY 366 IKWDVEDPRTLISWSLDKRIITWDLKDLEEEYAYPDGNEN - - - - SRRRAAVKIDPCLRF 367

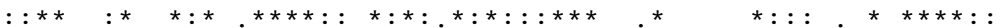

VHGGHTRRTNDFDIHPKIKNIFGSVGDDKLLEIWKPKTLPSDEPEQESADVQEDEEMKDA 423 VHGGHTRRTNDFDIHPKVKNIFGSVGDDKLLEIWKPKSLPGDEPEQESTEVQEDEEMKDA 469 IHGGHTNRINDFDIHTKVRNLFGSVGNDRLLEIWKPKTLPGDEPEEEEEEEVEEEALPEE 426 IHGGHTNRVNDFDVHPKIRSLYASVGDDNLLEVWK- - - - - - - - - - - - - - - 402

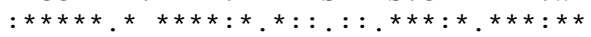

D - - AKGDKEGEEDSKMKD 439 E- - TKGVKDGEEDTKMKD 485 KKVSEETKESEQDVEMKD 444

Figure 2.2 Comparison of Candida albicans Hat2p protein with following species: C. dubliniensis, C. tropicales and $P$. stipitis

The comparison of the Hat2p protein length between C. albicans and other Candid spp. support our data. Fig. 2.2 shows the shorter length of the other Candida Hat2p proteins.

\section{Disruption of HAT1 in a hat2A mutant background strain}

Many yeast genes encode histone acetytransferase proteins in $C$. albicans but phenotypes of the putative mutants were unknown. We performed a phenotypic analysis of a yeast strain by deletion of HAT1 and HAT2 genes which encode members 
of the HAT families in C. albicans. We have deleted the ORF of the HAT1 gene by the “Ura-blaster" procedure (Fonzi \& Irwin, 1993) using protoplast method (Rose, 1990) in a hat $2 \Delta$ background strain.

Deletion was verified by using two pairs of primers for PCR analysis (data not shown) and by Southern blot analysis (Fig. 2.3B). The oligonucleotides used for this study are listed in Table 2.2. With the double mutant, we performed a whole set of experiments and below we describe the results obtained.

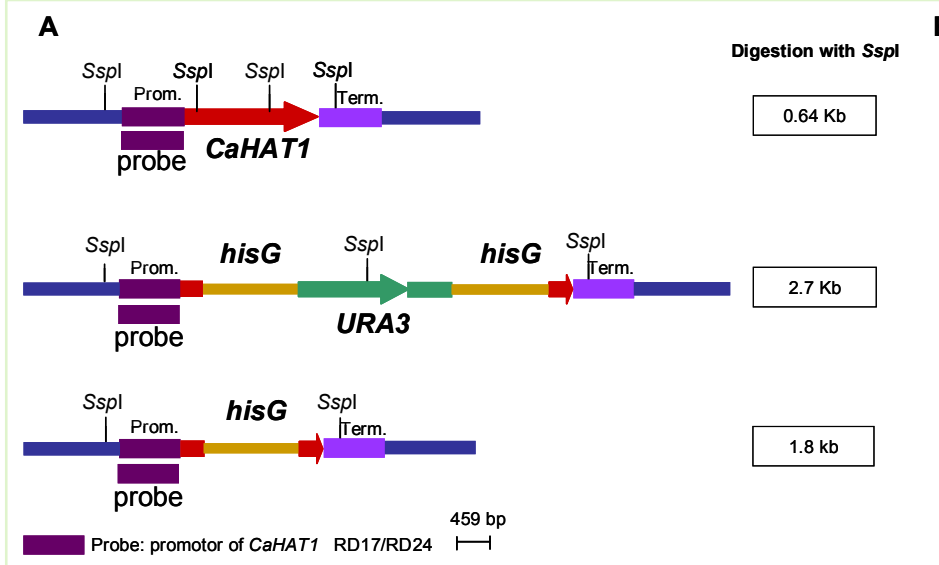

B

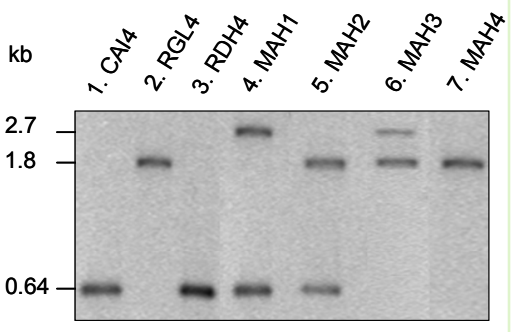

Figure 2.3 Deletion of CaHAT1 alleles. (A) Structure of different alleles. The wild-type HAT1 gene and the alleles disrupted by the hisG-URA3-hisG cassette or by hisG alone are shown. (B) Southern blot analysis of genomic DNA was performed with the following strains digested with Sspl.

Lane 1, CAI4 (HAT1/HAT1; HAT2/HAT2)

Lane 2, RGL4 (hat14::hisG/hat14::hisG; HAT2/HAT2)

Lane 3, RDH4 (HAT1/HAT1; hat24::hisG/hat24::hisG)

Lane 4, MAH1 (hat24::hisG/hat24::hisG; HAT2/hat24::hisGURA3hisG)

Lane 5, MAH2 (hat24::hisG/hat24::hisG; HAT1/hat14::hisG)

Lane 6, MAH3 (hat24::hisG/hat24::hisG; hat14::hisGURA3hisG/hat14::hisG)

Lane 7, MAH4 (hat24::hisG/hat24::hisG; hat14::hisG/hat14::hisG)

A 459 bp fragment of the CaHAT1 promoter was used as probe

The double mutant grew like hat $1 \Delta$ simple mutant, on solid and liquid media compared with the hat $2 \Delta$ and CAI4. To further analyze the growth rate of the double mutant, we performed a comparison of growth between all four strains: CAI4 (HAT1, HAT2) and RGL4 (hat1 $\Delta$ ), RDH4 (hat2 $\Delta$ ) and the MAH4 (hat1 $\Delta$, hat2 $\Delta$ ) double mutant.

The beginning of the exponential growth phase in the double mutant, as in the case of the hat1 $\Delta$ simple mutant, was delayed by up to $16 \mathrm{~h} \mathrm{(Fig.} \mathrm{2.4).} \mathrm{These} \mathrm{results} \mathrm{suggest}$ that HAT1 would be involved in vegetative growth in C. albicans. In our laboratory, we have been unable to detect any significant difference in growth rate, cell size and morphology between the hat $2 \Delta$ and CAl4 strains (Degano, Ph.D thesis in process). 


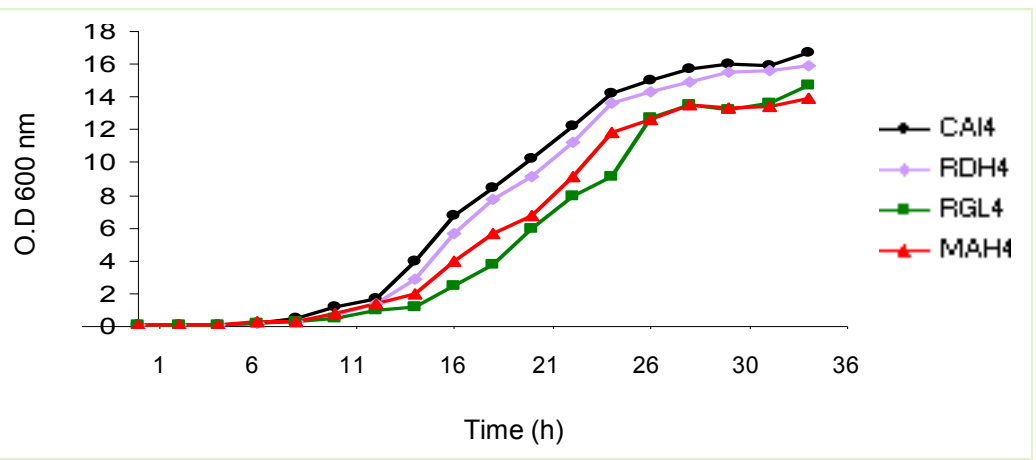

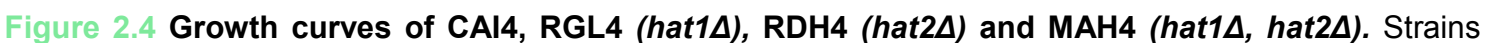
were pre-grown on rich medium containing $1 \%$ glucose and inoculated at an O.D at $600 \mathrm{~nm}$ of 0.1 YEPD. Growth was monitored over a period of $34 \mathrm{~h}$ by measuring the optical density of the cultures.

Morphologically, the double mutant, like the hat $1 \Delta$ simple mutant, is very different to the CAI4 strain as shown in Fig. 2.5. The culture of the hat $1 \Delta$, hat2 $\Delta$ double mutant in YEPD liquid medium was composed of a mixture of large cells and large chains of elongated, aberrantly shaped cells attached to one another suggesting that Hat1p activate or suppress transcription of genes that directly or indirectly control cell morphology.

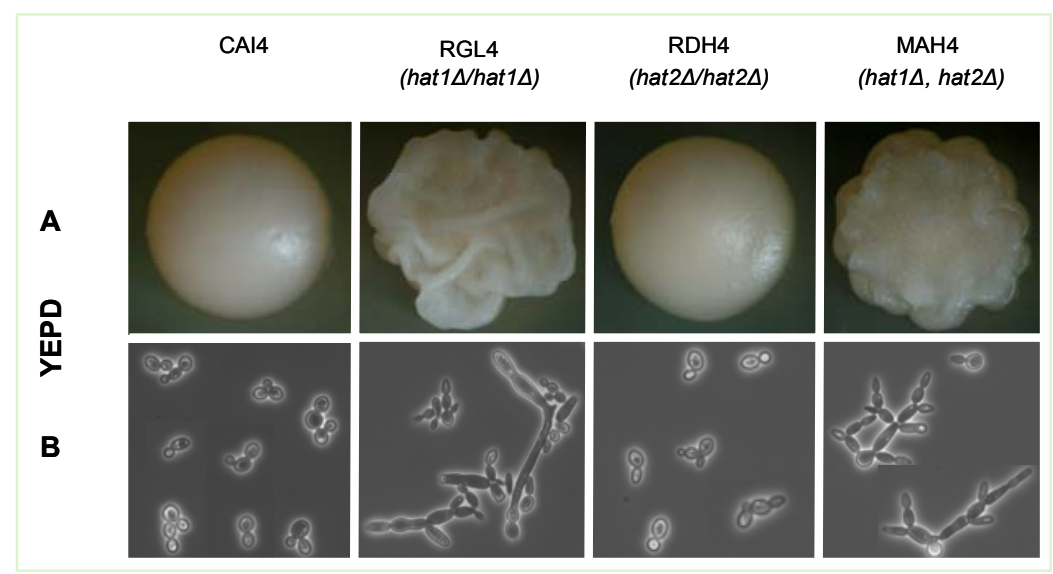

Figure 2.5 (A) Colony growth and (B) morphological characteristics of $C$. albicans homozygous strain CAI4, RGL4, RDH4 and MAH4 in YEPD media

\section{Behavior of the hat1 $\Delta$, hat2 $\Delta$ double mutant in solid media}

For the observation of the filamentous growth on solid media, cells of the simples and double mutant as well as the CAl4 strain were plated at the 50 ufc on plates. Solid media normally induces filamentous growth after 3 days. The double and the hat1 $\Delta$ simple mutants displayed hyperfilamentation, whereas hat $2 \Delta$ cells formed hypha like the wild-type parent under these conditions (Fig. 2.6). These results indicated that the alternative pathway was almost fully induced in a hat $1 \Delta$ simple and hat $1 \Delta$, hat $2 \Delta$ double mutant. A similar morphological phenotype has been reported in the efh1 $1 \Delta$, 
efg1s double mutant (Doedt et al., 2004). We also observed that, like in both simple mutants, the double mutant was also able to form hypha in Embedded medium (Fig. 2.6).

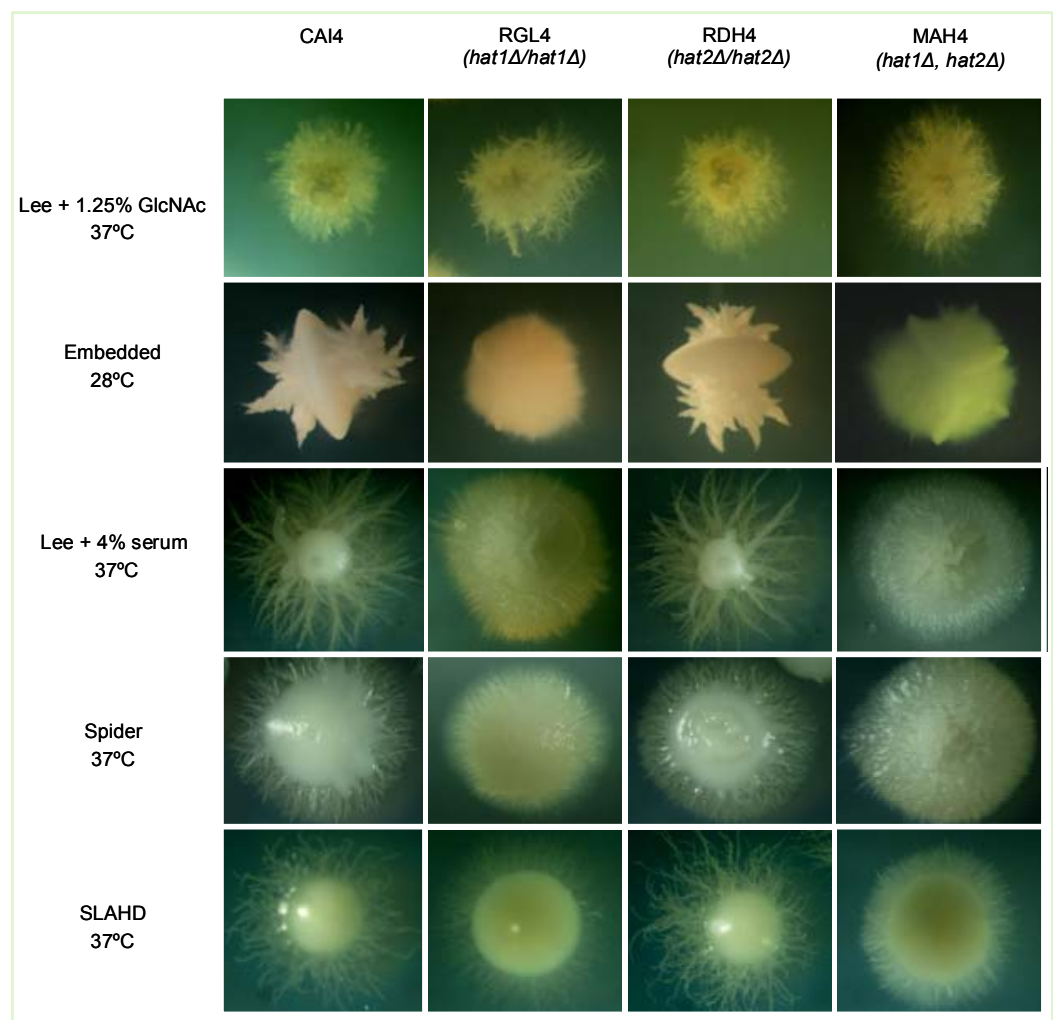

Figure 2.6 Growth of the CAI4, RGL4, RDH4 and MAH4 on solid media. All strains were grown on solid medium which induce hyphal development. The plates were incubated for 4 days at $37^{\circ} \mathrm{C}$.

\section{Behavior of the hat1 $\Delta$, hat2 $\Delta$ double mutant in liquid media}

The behavior of the double mutant strain was next investigated by assessing hyphal formation in Lee $\left(\mathrm{pH} 6.8,37^{\circ} \mathrm{C}\right.$ ) (data not shown) or in serum inducing media.

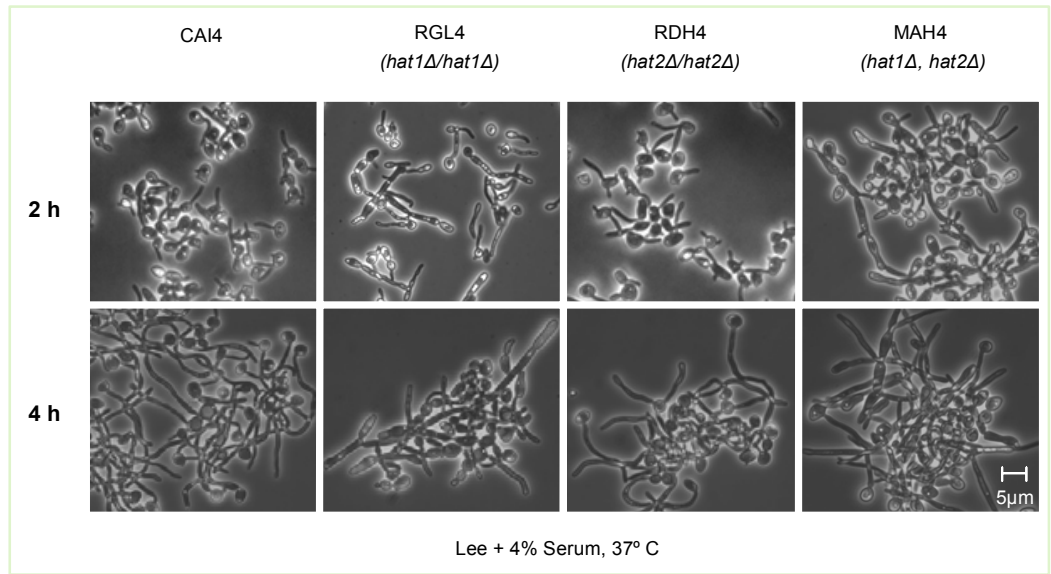

Figure 2.7 Germinative behavior of CAI4, RGL4, RDH4 and MAH4 strains during the yeast-hypha transition in Lee $+4 \%$ serum at $37^{\circ} \mathrm{C}$ 
Fig. 2.7 shows that whereas CAI4 and hat2 $\Delta$ mutant formed normal hypha, hat $1 \Delta$, hat $2 \Delta$ double mutant like hat $1 \Delta$ simple mutant have a block of hypha formation in both medium and an aberrant morphology with long, thick, stubby protrusions resembling pseudohypha were observed. A similar phenotype has been reported previously in our laboratory in the hat $1 \Delta$, hda1 $1 \Delta$ double mutant.

\section{Chlamydospore formation}

We try to determine whether the deletion of both genes might influence chlamydospore formation. We analyzed the growth of the double mutant in the presence of the cornmeal agar. The results are shown in Fig. 2.8. The hat $1 \Delta$, hat $2 \Delta$ double mutant like both simple mutants were able to formed chlamydospore. These results suggesting that deletion of the HAT1 and HAT2 genes did not lead to an apparent defect in chlamydospore formation. It has been reported that other histone acetyltransferase like GCN5 failed to form chlamydospore (Degano, Ph.D thesis in process). Collectively, our data indicate that a hat $1 \Delta$ and hat $1 \Delta$, hat $2 \Delta$ mutation does not influence the various morphogenetic processes that are strongly affected by a gcn5s mutation (morphogenesis and phenotypic switching).
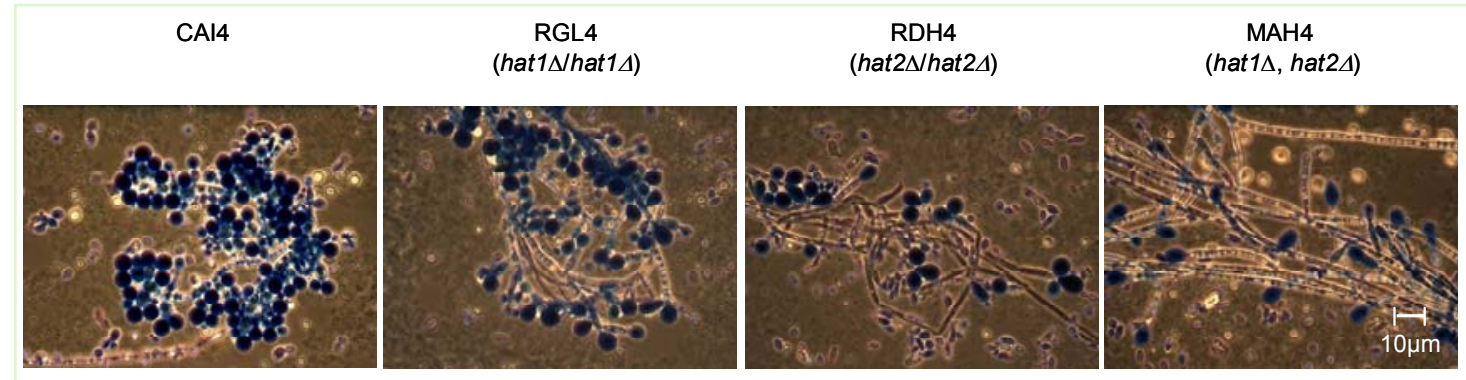

Figure 2.8 Hat $1 p$ and Hat2p are not required for chlamydospore formation. The strains were streaked out lightly on chlamydospore induction medium (cornmeal agar (Difco)-0.33\% Tween 80.

\section{Phenotype analysis of the hat1 $\Delta$, hat2 $\Delta$ double mutant}

To gain further characterize and better define of both simples and double mutant, we carried out further phenotypic tests including sensitivity to SDS, $\mathrm{NaCl}, \mathrm{CFW}$, Caffeine, Amphotericin B, Hygromycin, Fluconazole, Itraconazole and Congo red. To do so, the sensitivity of both simple and the hat $1 \Delta$, hat $2 \Delta$ double mutant was compared with that of the CAI4. We found that the hat $1 \Delta$ and double mutant were more sensitive than the hat2 $\Delta$ and the CAI4 strain when they were incubated in the presence of $1 \mathrm{M}$ of $\mathrm{NaCl}, 1$ $\mathrm{mg} / \mathrm{ml}$ of Calcofluor white (CFW), $0.5 \mathrm{mg} / \mathrm{ml}$ of Congo red and $15 \mathrm{mM}$ of Caffeine (Fig. 2.9). The sensitivity of a hat $1 \Delta$, hat $2 \Delta$ double mutant was similar to that of the single 
mutants and the CAI4 strain in the medium supplemented with SDS (0.05\%). In the present of Amphotericin B, Fluconazole and Itraconazole, the hat $1 \Delta$ and double mutant were showed a moderate sensitivity when compared to that of hat $2 \Delta$ and CAl4. These results suggest that Hat1p maybe control expression some of cell wall genes.

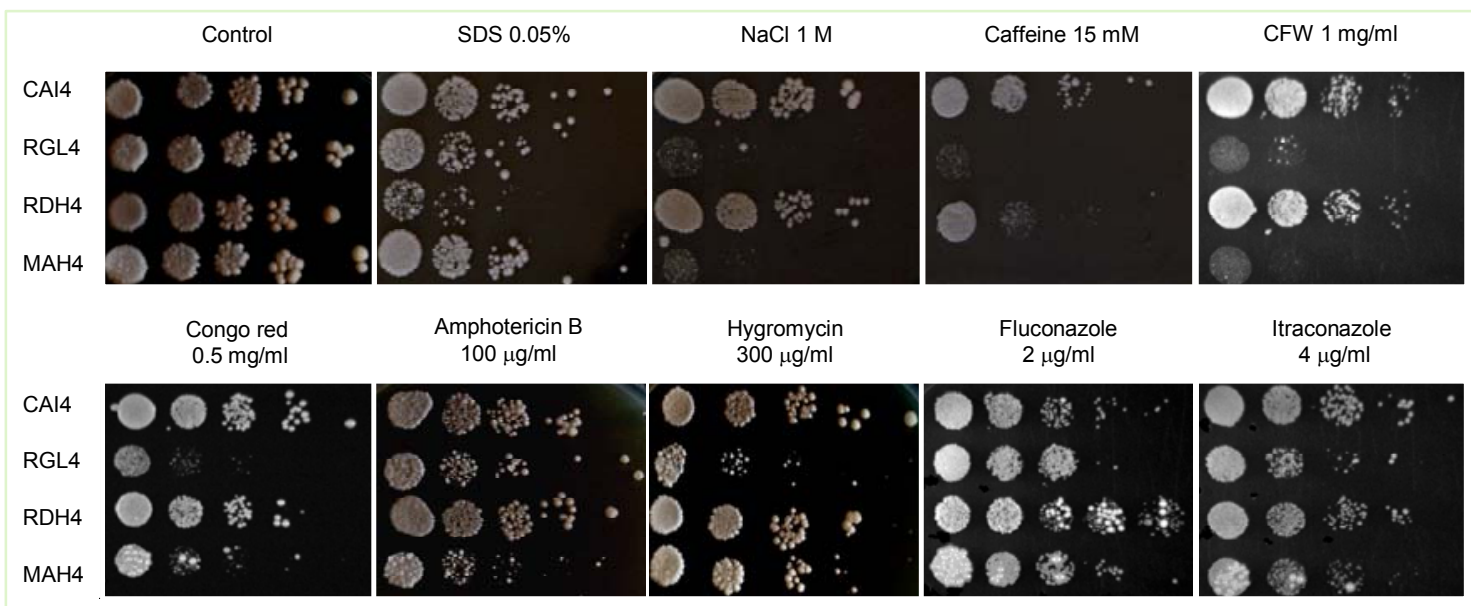

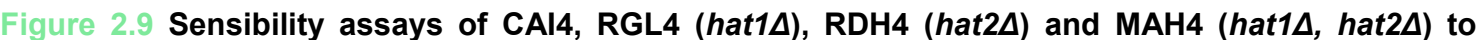
different agents. All strains were grown in liquid YEPD. The optical density at $600 \mathrm{~nm}$ of the cultures were adjusted to 0.4 with the same medium and $5 \mu \mathrm{l}$ aliquots from the cultures and from 10 -fold serial dilutions were spotted onto YEPD plates supplemented with different agents. Plates were incubated at $28^{\circ} \mathrm{C}$ for 4 days.

The sensitivity of yeast cells to Zymolyase has been used to detect changes in cell wall composition and arrangement (Ram et al., 1994). To address this issue, we monitored the response of all strains to the presence of the Zymolyase 20T for 70 minutes at $30^{\circ} \mathrm{C}$. We found that like the hat $1 \Delta$, the double mutant, are slightly more sensible to Zymolyase than the hat2 $\Delta$ and CAI4 strain (Fig. 2.10).

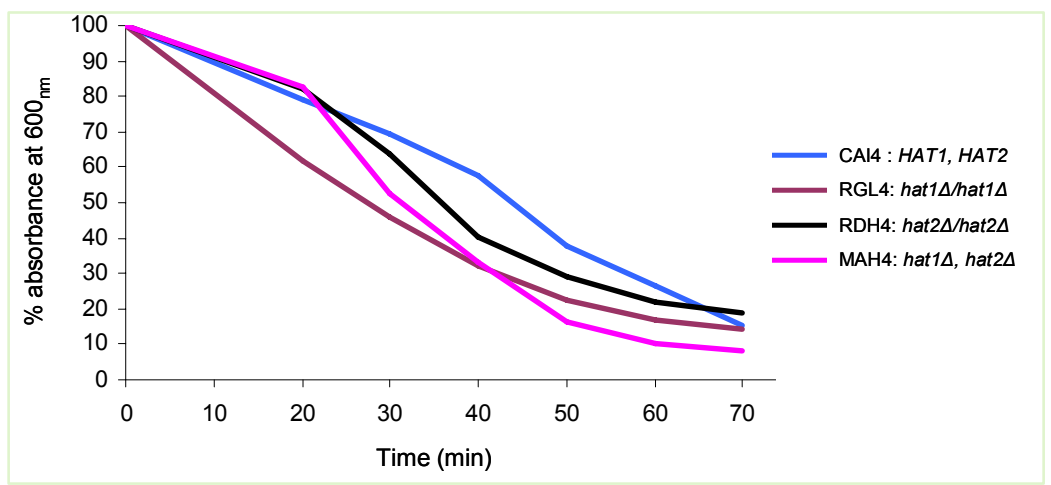

Figure 2.10 Resistance to a cell wall-degrading enzymatic complex of $\boldsymbol{C}$. albicans. All strains were grown until the exponential phase in YEPD, at $28^{\circ} \mathrm{C}$. Aliquots $\left(2 \times 10^{7}\right.$ cells $)$ were resuspended in $10 \mathrm{mM}$ Tris-Hcl PH 7.5 and treated with $10 \mu \mathrm{g}$ of Zymolyase per ml. The decrease in optical density (O.D) (percentage of resistant cells) is monitored as described in Methods. 


\section{Alcian blue binding assay}

Next we determined the level of Alcian blue binding to CAI4 and to the three mutant strains of $\boldsymbol{C}$. albicans, using the method previously described (Herrero et al., 2002). As shown in Fig. 2.11, the level of Alcian blue binding was reduced to $48.14 \%$ in the hat1 $\Delta$ mutant. In the hat $2 \Delta$ mutant a small increase was detected and the double mutant present a similar level of Alcian blue binding to the CAl4 strain suggesting a compensatory effect of the HAT2 deletion on the hat1 $\triangle$ mutation.

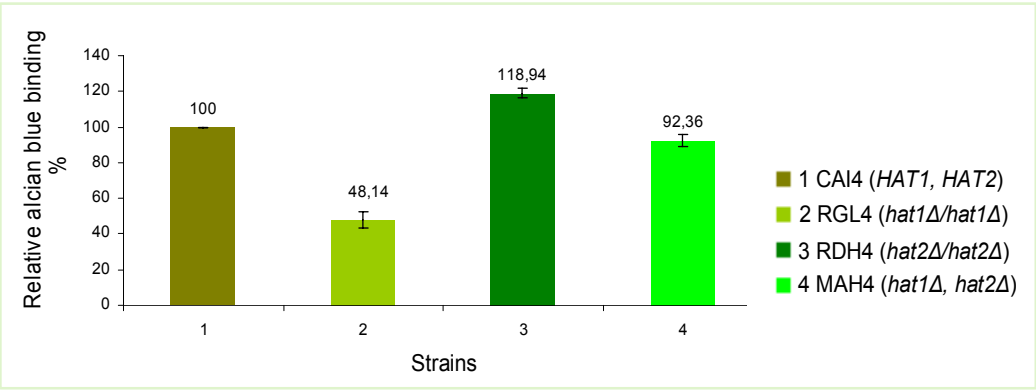

Figure 2.11 Comparison of Alcian blue binding of double mutant with parental and simple mutants. Relative dye binding of MAH4 was calculated as the percentage of dye bound compared with results for the parental strains (RGL4, RDH4 and CAI4). Results are average of three independent determinations; bars indicate standard deviations.

\section{Virulenece assay}

We decided to determine whether the absence of the Hat1p and Hat2p might lead to a defect in virulence in a mouse model. We tested the virulence of the $C$. albicans hat mutant strains in a mouse model infection because hyphal growth is hypothesized to be important for the pathogenicity of C. albicans. Isogenic URA3 strains were used because ura3 strains have reduced virulence (Cole et al., 1995; Leberer et al., 1996). All of the mice injected with the CAI4 and hat2 $\Delta$ mutant died by day 3 (Fig. 2.12).

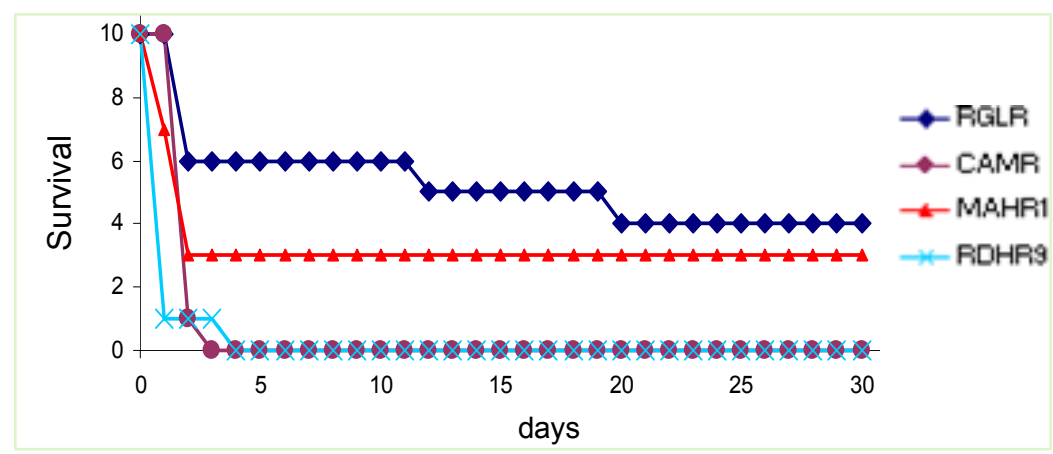

Figure 2.12 C. albicans double and hat $1 \Delta$ simple mutants are hypovirulent in a mouse model. Survival curves for mice. ( $\mathrm{n}=10$ for each $C$. albicans strain at each inoculation dose) infected with $5 \times 10^{8}$ cells of $C$.

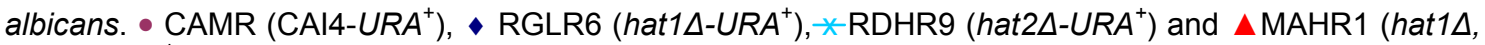
hat2 $\triangle-U R A^{+}$). 
In contrast, the hat $1 \Delta$ and hat $1 \Delta$, hat $2 \Delta$ homozygotes were less virulent; $40 \%$ and $30 \%$ of the mice were alive at the end of the experiment respectively. Similar to the results was reported for the gcn5 $\Delta$, hat1 $\Delta$ double mutant (Rashki, Ph.D thesis 2009).

\section{Cellular location of the Hat1p and Hat2p proteins}

In order to determine the cellular location of the Hat1p and Hat2p in the C. albicans, we constructed strains that carried Hat1-GFP and Hat2-GFP fusion proteins and observed it under the fluorescence microscope. We found that the two GFP fused proteins were mainly located in the nucleus (Fig. 2.13 and Fig. 2.14). A transformed with a construct of the GFP not in phase wild-type strain was used as a negative control for both proteins.

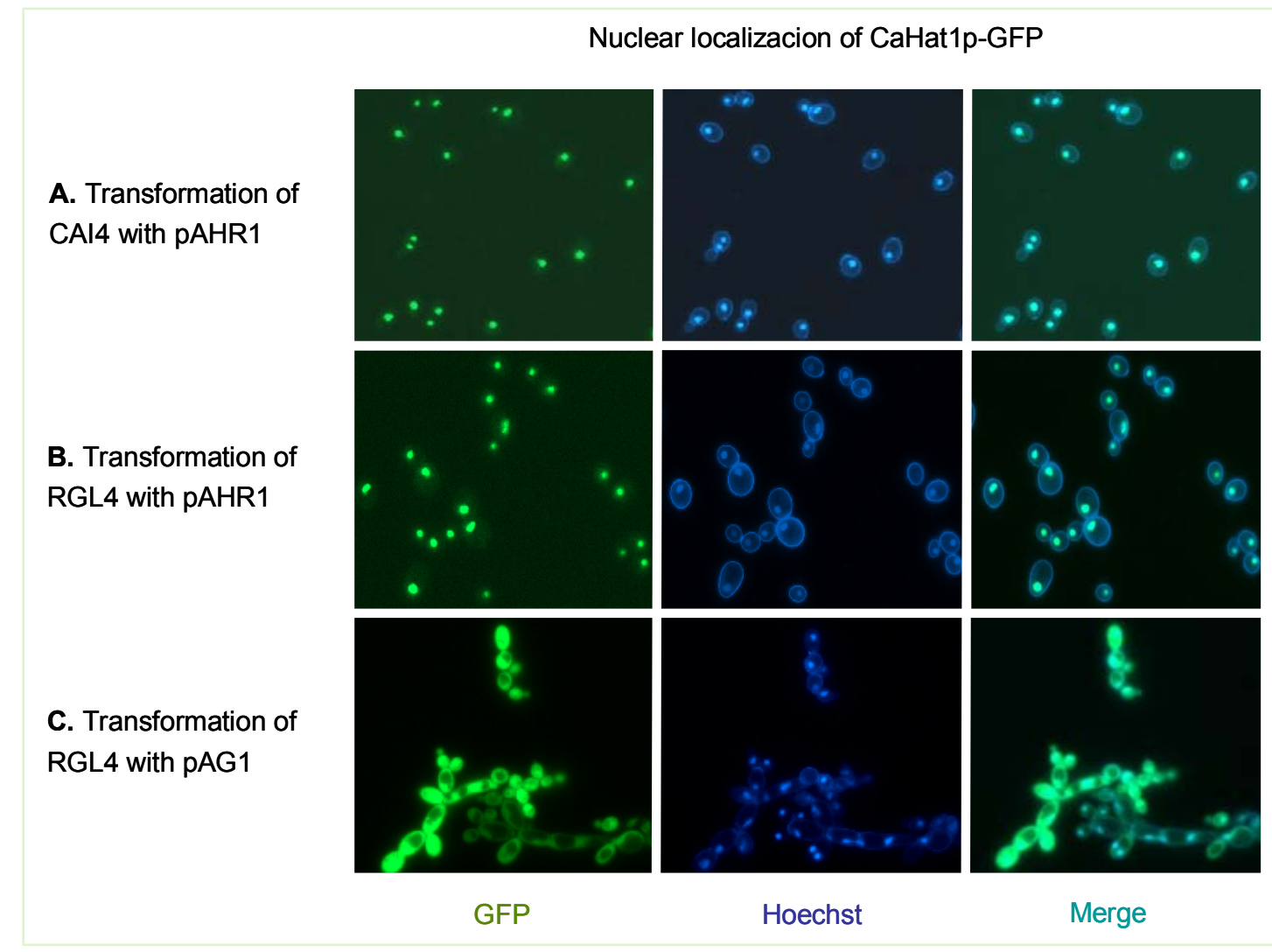

Figure 2.13 Subcellular localization of Hat1-GFP in wild-type and mutant strains. Localization of the fusion protein was visualized by fluorescence microscopy. Nuclei were visualized by Hoechst staining. 
Nuclear localizacion of CaHat2p- GFP

A. Transformation of CAI4 with pZRG1

B. Transformation of $\mathrm{RDH} 4$ with pZRG1

C. Transformation of $\mathrm{RDH} 4$ with $\mathrm{pAG} 1$
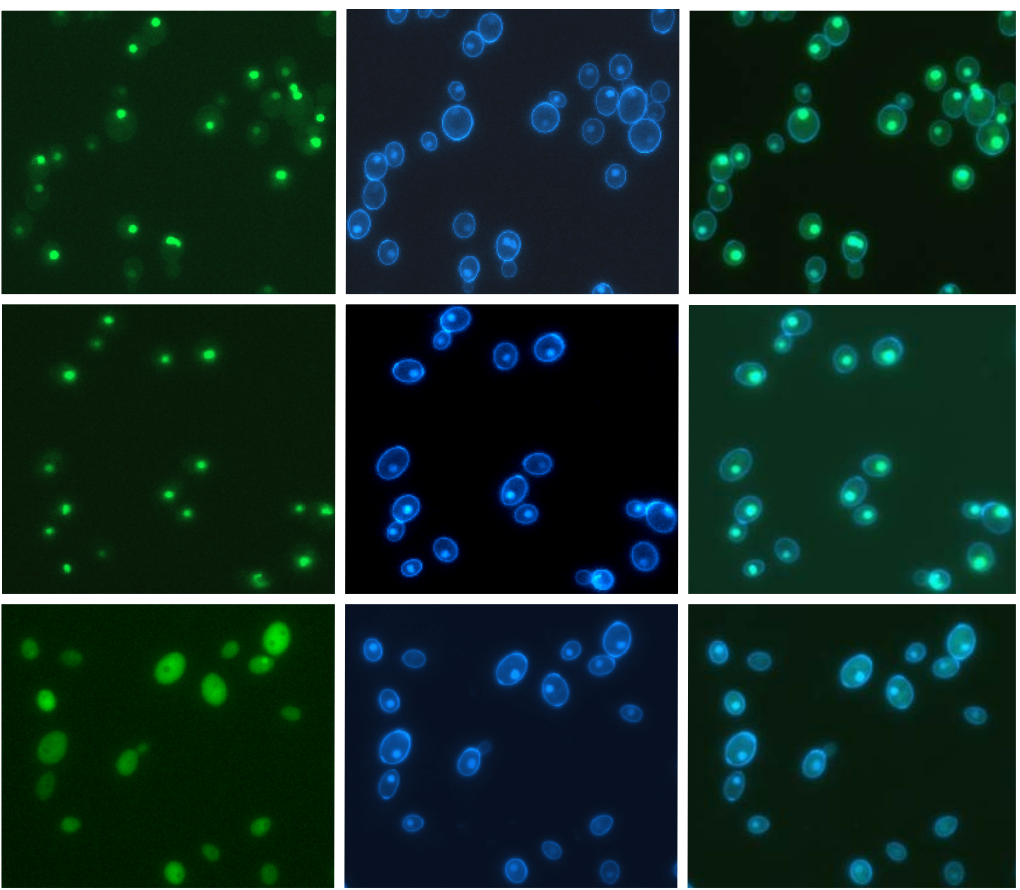

GFP

Hoechst

Merge

Figure 2.14 Subcellular localization of Hat2-GFP in wild-type and mutan strains. Localization of the fusion protein was visualized by fluorescence microscopy. Nuclei were visualized by Hoechst staining.

Next, we asked whether their nuclear residence is interdependent. To address this question, Hat1-GFP and Hat2-GFP were visualized in the Candida cells defective in any of the other companion protein. The nuclear residence of Hat1-GFP exhibited no difference between cells with a wild-type and hat $2 \Delta$ genetic background, suggesting that Hat2p is not necessary for localization of that Hat1p in the nucleus (Fig. 2.15A).

We also analyzed location of Hat $2 p$ in a hat $1 \Delta$ mutant. In this case, we transformed a plasmid that carrying GFP-fused Hat2p. As shown in Fig. 2.15B, we detected that the GFP-Hat2 $p$ signal localized in the cytoplasm suggesting that Hat $1 p$ is necessary for localization of the Hat2p in the nucleus.

All these results strongly suggested that Hat $1 p$ would interact directly o indirectly with the Hat2p in a histone acetyltransferase complex.

In order to see whether Hat1p was directly interacting with Hat2p, we performed the standard two-hybrid assays in $S$. cerevisiae. For this purpose, we tested fusions of Hat1p or Hat2p to the Gal4p DNA-binding (BD) or activating domains (AD). We found that Hat1p didn't interact directly with Hat2p (result not shown) suggesting that maybe other protein o proteins are involved in a bridge between Hat1p and Hat2p. 
A. Transformation of pAHR1 (GFP-Hat1) in the RDH4 (hat2L)
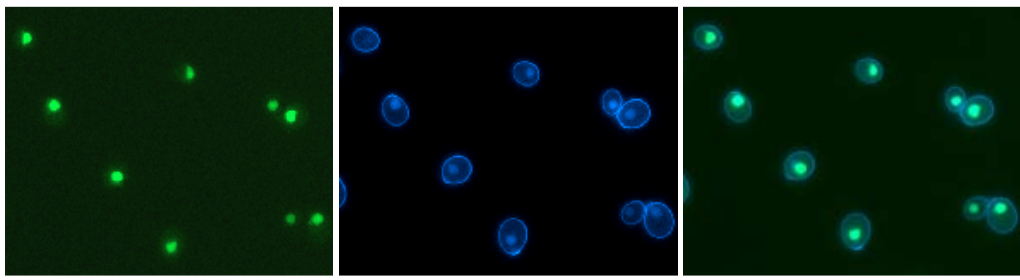

B. Transformation of pZRG1 (GFP-Hat2) in the RGL4 (hat1A)

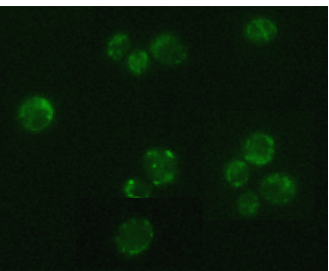

GFP

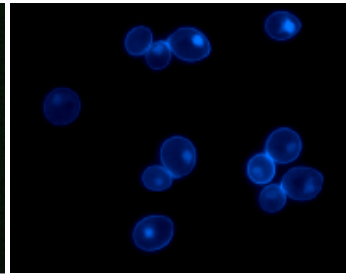

Hoechst

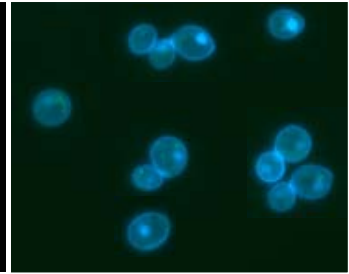

Merge

Figure 2.15 Subcellular localization of Hat1-GFP in the hat2 $\Delta(\mathrm{A})$ and Hat2-GFP in the hat1 $\Delta$ mutant $(\mathrm{B})$. Localization of the fusion protein was visualized by fluorescence microscopy. Nuclei were visualized by Hoechst staining.

\section{Transcriptional profile of the hat $2 \Delta$ mutant during the yeast mode of growth}

We have analysed the transcriptional profile of the hat $2 \Delta$ mutant while the transcription profile of the hat1 $1 \Delta$ mutant has been previously performed in our laboratory. The experiments were done in duplicate by using two different exponentially growing YNB culture for each mutant. In the hat $2 \Delta$ mutant, we detected an altered expression of 109 genes: 26 genes being up-regulated and 83 being down-regulated. It has been reported that 62 genes were affected in the hat1 $1 \Delta$ mutant ( 45 genes up- and 17 downregulated). Only two genes (HHF1 and STF2) were commonly up-regulated in both single mutants suggested that transcription profile of the hat $1 \Delta$ and hat $2 \Delta$ are different during the yeast mode of growth and thus demonstrating that both acetyltranferases regulate a different set of genes.

The major gene categories of HAT2-dependent genes down-regulated during the yeast mode of growth included cell wall proteins (5 genes), genes encode for elongation factor (6 genes), ribosomal proteins (10 genes), stress response (4 genes) and transcription factors (4 genes) (Table 2.3). Seven genes involved in respiration (ATP6, COX3A, COX3B, NAD2, NAD5, NAD6 and STF2), three genes encoding known histone assembly proteins and two genes encoding for ribosomal proteins were found to be up-regulated in the yeast mode of growth (Table 2.3). Eleven genes involved in glycolysis and TCA cycle (CDC19, PDB1, FBA1, PFK2, PYC2, ACO1, ACO2, CIT1, GDH3, MDH1-1 and MIS11) (McAlister \& Holland, 1985; Overkamp et al., 2002: 
Reifenberger et al., 1995; Sonneborn et al., 2000; Walsh et al., 1983) were found to be down-regulated in the hat2 $\triangle$ mutant. Expression of ACO1, ACO2, CIT1, MIS1 and PYC2 genes has been reported to be decreased in the hat1 $\triangle$ mutant suggesting that this group of genes were regulated separately by Hat1p and Hat2p. Six genes encoding for translation elongation factors (CAM1-1, CEF3, EFB1, EFT2, TEF1 and TIF1) (Lorenz et al., 2004; Maneu et al., 1996; Mirbod et al., 1996; Myers et al., 1992) also were down-regulated in the hat $2 \Delta$ mutant. Although their roles in the yeast mode of growth in $C$. albicans are unknown, it has been reported that transcription of translation-related genes are down-regulated upon phagocytosis (Liu et al., 2005; Lorenz et al., 2004).

Table 2.3 Differentially expressed genes in hat $2 \Delta$ mutant during the yeast mode of growth

\begin{tabular}{|c|c|c|c|}
\hline Gene name & ORF & Function & Fold regulation \\
\hline \multicolumn{4}{|c|}{ Up-regulated genes } \\
\hline ATP6 & - & Subunit6 of the F0 sector of mitochondrial F1F0 ATP & 1.77 \\
\hline COX3A & - & $\begin{array}{l}\text { synthesis } \\
\text { Subunit III of cytochrome c oxidase }\end{array}$ & 3.31 \\
\hline COX3B & - & Subunit III of cytochrome c oxidase & 2.49 \\
\hline NAD2 & - & Subunit 2 of NADH: ubiquinone oxidoreductase & 2.02 \\
\hline NAD5 & - & A multisubunit enzyme complex (complex I) & 1.64 \\
\hline NAD6 & - & Subunit 6 of NADH: ubiquinone oxidoreductase & 1.91 \\
\hline STF2 & orf19.2107.1 & Protein involved in ATP biosynthesis & 2.19 \\
\hline \multicolumn{4}{|c|}{ Histone assembly } \\
\hline HHF1 & orf19.1059 & Putative histone $\mathrm{H} 4$ & 2.04 \\
\hline HTA1 & orf19.6924 & Putative histone $\mathrm{H} 2 \mathrm{~A}$ & 1.75 \\
\hline NHP6A & orf19.4623.3 & Protein described as nonhistone chromatin component & 2.36 \\
\hline \multicolumn{4}{|c|}{ Ribosomal proteins } \\
\hline RPL26A & orf19.3690.2 & Ribosomal protein & 1.71 \\
\hline RPL40B & orf19.4684.2 & $\begin{array}{l}\text { Protein with similarity to the ribosomal protein of } S \text {. } \\
\text { cerevisiae Rpl } 40 \mathrm{Bp}\end{array}$ & 1.72 \\
\hline \multicolumn{4}{|c|}{$\begin{array}{l}\text { Down-regulated genes } \\
\text { Glycolisis }\end{array}$} \\
\hline CDC19 & orf19.3575 & Putative pyruvate kinase & 0.45 \\
\hline PDB1 & orf19.5294 & Similar to pyruvate dehydrogenase & 0.63 \\
\hline FBA1 & orf19.4618 & Putative fructose-bisphosphate aldolase & 0.63 \\
\hline PFK2 & orf19.6540 & Beta subunit of phosphofructokinase & 0.59 \\
\hline PYC2 & orf19.789 & Putative pyruvate carboxylase & 0.43 \\
\hline \multicolumn{4}{|l|}{ TCA cycle } \\
\hline $\mathrm{ACO} 1$ & orf19.6385 & Protein described as aconitase & 0.56 \\
\hline $\mathrm{ACO} 2$ & orf19.6632 & Protein described as aconitate hydratase 2 & 0.60 \\
\hline CIT1 & orf19.4393 & Protein described as citrate synthase & 0.60 \\
\hline GDH3 & orf19.4716 & Similar to NADp-glutamate dehydrogenase & 0.52 \\
\hline $\mathrm{MDH} 1-1$ & orf19.4602 & Predicted malate dehydrogenase & 0.51 \\
\hline MIS11 & orf19.2364 & Putative protein of glycine catabolism & 0.57 \\
\hline \multicolumn{4}{|c|}{ Ribosomal proteins } \\
\hline RPL8B & orf19.2311 & Predicted ribosomal protein & 0.55 \\
\hline RPL13 & orf19.2994 & Protein described as ribosomal subunit & 0.59 \\
\hline RPL16A & orf19.6085 & Similar to S. cerevisiae ribosomal protein Rpl16ap & 0.64 \\
\hline RPL81 & orf19.6002 & 60 s ribosomal protein $17 a . e . b$ & 0.52 \\
\hline RPPO & orf19.7015 & Putative ribosomal protein & 0.27 \\
\hline RPS4A & orf19.5341 & Predicted ribosomal protein & 0.51 \\
\hline RPS15 & orf19.5927 & Putative ribosomal protein & 0.42 \\
\hline RPS23A & orf19.13632 & Putative ribosomal protein & 0.63 \\
\hline RPS8A & orf19.6873 & Putative ribosomal protein & 0.34 \\
\hline YST1 & orf19.6975 & Ribosome-associated protein & 0.57 \\
\hline \multicolumn{4}{|c|}{ Elongation factor } \\
\hline CAM1-1 & orf19.2651 & Putative translation elongation factor & 0.53 \\
\hline CEF3 & orf19.11629 & Translation elongation factor 3 & 0.47 \\
\hline EFB1 & orf19.3838 & Translation elongation factor ef- 1 beta & 0.56 \\
\hline
\end{tabular}




\begin{tabular}{|c|c|c|c|}
\hline $\begin{array}{l}\text { EFT2 } \\
\text { TEF1 } \\
\text { TIF1 }\end{array}$ & $\begin{array}{l}\text { orf19.5788 } \\
\text { orf19.9009 } \\
\text { orf19.3324 }\end{array}$ & $\begin{array}{l}\text { Elongation factor } 2 \text { (eEF2) } \\
\text { Translation elongation factor 1-alpha } \\
\text { Similar to S. cerevisiae translation initiation factor }\end{array}$ & $\begin{array}{l}0.61 \\
0.55 \\
0.53\end{array}$ \\
\hline \multicolumn{4}{|c|}{ ( } \\
\hline PGA45 & orf19.2451 & Putative GPI-anchor & 0.62 \\
\hline PGA59 & orf19.2767 & Putative GPI-anchored protein of unknown function & 0.29 \\
\hline PGA62 & orf19.10281 & Putative GPI-anchored protein & 0.24 \\
\hline TOS1 & orf19.1690 & Similar to alpha agglutinin anchor subunit & 0.55 \\
\hline YWP1 & orf19.3618 & Putative GPI-anchor; cell wall and secreted & 0.17 \\
\hline \multicolumn{4}{|c|}{ Stress response } \\
\hline SOD1 & orf19.2770.1 & $\begin{array}{l}\text { Cytosolic copper- and zinc-containing superoxide } \\
\text { dismutase }\end{array}$ & 0.62 \\
\hline SOD2 & orf19.3340 & $\begin{array}{l}\text { Mitochondrial manganese-containing superoxide } \\
\text { dismutase }\end{array}$ & 0.57 \\
\hline SSB1 & orf19.6367 & Putative heat shock protein & 0.32 \\
\hline HSP90 & orf19.6515 & Chaperon of Hsp90p family & 0.57 \\
\hline \multicolumn{4}{|c|}{ Transcription factors } \\
\hline RGT1 & orf19.2747 & Transcriptional repressor & 0.56 \\
\hline SKO1 & orf19.1032 & Putative transcription factor & 0.61 \\
\hline EFG1 & orf19.8243 & Transcriptional repressor & 0.31 \\
\hline NRG1 & orf19.7150 & Transcriptional repressor & 0.59 \\
\hline
\end{tabular}

\section{Transcriptional profile of the hat $2 \Delta$ during the yeast-hypha transition}

Next, we focused on the identification of genes whose expression was altered in the hat $2 \Delta$ mutant strain during the yeast-hypha transition. We generated the transcriptional profile for the hat $2 \Delta$ mutant in Lee medium for 15,60 and 180 min at $37^{\circ} \mathrm{C}$ using the CA14 strain as reference. In a previous study in our laboratory, the transcriptional profile of hat1 $1 \Delta$ has been performed after growth in hypha-inducing condition at the same times. A total of 429 genes were found to change at the consensus level of $\geq 1.5$ fold, including 196 up- and 233 down-regulated genes. The distribution of genes altered at the three points is represented in the Venn diagram, and the clustering of genes regulated by deletion of HAT2 is represented in Fig. 2.16. We found that 2 genes (up-regulated) were common to the three times assayed, 54 genes at 15 and 60 min (35 genes up- and 19 down-) and 11 genes at 15 and 180 min ( 9 up- and 2 down-). 156 genes (85 up- and 71 down-), 167 genes (40 up- and 127down-) and 39 genes (25 up- and 14 down-) varied only at one time of induction (at 15, 60 and $180 \mathrm{~min}$ respectively). Only 2 genes $B U D 7$ encoding a protein with similarity to the $B C H 1$ gene of S. cerevisiae that has a role in cellular bud site selection (Braun et al., 2005) and MEF2 a mitochondrial elongation factor, similar to Mef2p of $S$. cerevisiae involved in translational elongation (Rasmussen, 1995) were up-regulated commonly during three times of incubation. 


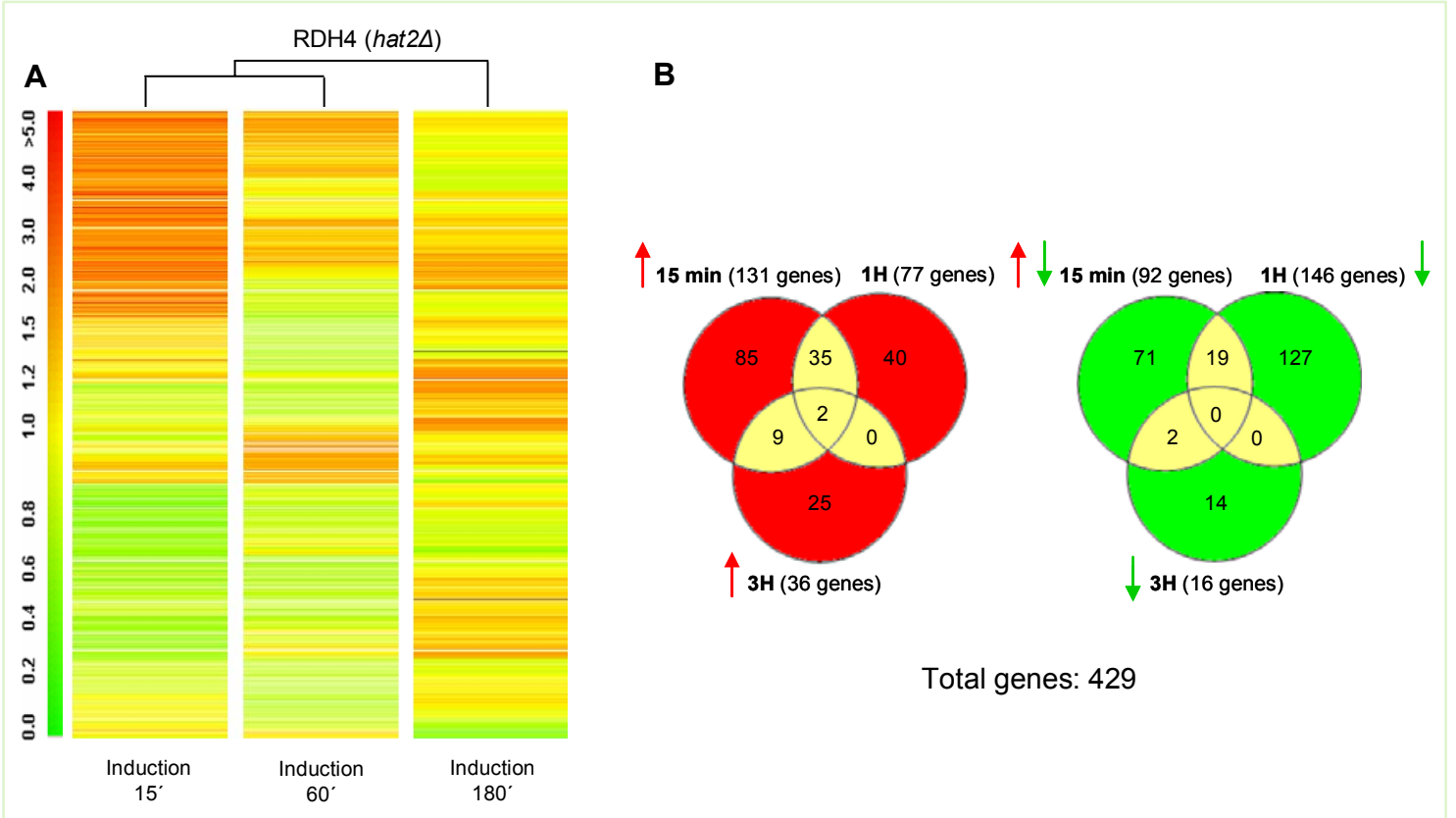

Figure 2.16 Transcript profiles of $C$. albicans hat2 $\Delta$ mutant during the yeast-hypha transition $(A)$. Venn diagrams of genes regulated by Hat2p in the RDH4 (B). The CAl4 strain was used as reference, which all were grown in identical conditions: Lee $/ 37^{\circ} \mathrm{C}$ (inducing).

\section{Time course of gene induction of the main significant categories}

The genes belonging to diverse functional categories whose expression level changed at least at one time of incubation were clustered together. These diverse functional categories include genes that are both up- and down-regulated, indicating a differential regulation of these functions by the hat $2 \Delta$ mutant. Many genes can not be readily categorized into functional groups and represent a wide range of cellular metabolic processes (Supplementary tables 2.3 and 2.4). The microarray results demonstrate that the lack of Hat2p affect a large number of cells functions and most likely serve to globally reprogram in $C$. albicans physiology to allow the hat $2 \Delta$ mutant to adapt to this environment.

\section{Expression of transcription factors}

Only one transcription factor was down-regulated at the 15 and 60 min of incubation; namely, ZPR1, which has been described as a putative zinc finger transcription factor with unspecified role in morphogenesis, regulated by Gcn4p and repressed in response to amino acid starvation (Rogers \& Barker, 2003; Yin et al., 2004). Six genes including IPF19804 (Karababa et al., 2004) (at 15 min), MRR1 (Morschhauser et al., 2007) (at 60 $\mathrm{min}$ ), three transcription factors member of a family of telomere-proximal genes CTA2, CTA24, CTA29 (Doedt et al., 2004; Kaiser et al., 1999; van het Hoog et al., 2007; Zakikhany et al., 2007), GAL4 (Martchenko et al., 2007) (at $180 \mathrm{~min}$ ) and HAC1 (Nobile 
\& Mitchell, 2005), TAF145 (Davis et al., 2002), SPT20 (Marchais et al., 2005), ZCF20 (Yan et al., 2008) (at 15 min), CAP1 (Alarco et al., 1997), CPH1 (Chen et al., 2002; Magee et al., 2002), CTA4 (Kaiser et al., 1999), CTA8 (Kaiser et al., 1999), MIG1 (Garcia-Sanchez et al., 2004), TBP1 (Leng et al., 1998), TUP1 (Murad et al., 2001a) (at $60 \mathrm{~min}$ ) were up- and down-regulated respectively.

Only HAC1 has been reported to be down-regulated in the hat1 $\triangle$ mutant at 15 min of incubation suggesting that the hat $2 \Delta$ mutant has a distinct transcriptome of hat1 $\Delta$ mutant implying that Hat2p has additional functions in the cell. Among the uncharacterized ORFs without significant homologies to $S$. cerevisiae, two ORF (CTA2 and CTA24) which were named TLO3 and TLO12 are putative members of a family of telomeric proximal genes In the Candida Data Base.

Cell wall genes: Our results indicate that the transcript levels of seven encoding for cell wall proteins including BMT5 (at $15 \mathrm{~min}$ ), IRS4, FGR41 (at $60 \mathrm{~min}$ ), HWP1, PGA54 (at $180 \mathrm{~min}$ ), BMT9, CHS2 and GSL1 (at 15 and $60 \mathrm{~min}$ ) and PGA45 (at 15 and 180 min) were up-regulated and expression of PHR2, TOS1 (at $15 \mathrm{~min}$ ), ALS4, ECM31, HYR3, PGA6, PGA63 (at $60 \mathrm{~min}$ ), HYR1 (at $180 \mathrm{~min}$ ) and GSC1 (at 15 and $60 \mathrm{~min}$ ) were down-regulated (Table 2.4).

Only HWP1, TOS1 (at $60 \mathrm{~min}$ ) and ALS4 (at $60 \mathrm{~min}$ ) have been reported to be downand up-regulated in the hat1 $\Delta$ mutant suggesting again that the hat $2 \Delta$ mutant has a distinct transcriptome of hat1 $\triangle$ mutant. Expression of HWP1, PGA54 (at 15 min), HYR1 (at 15 and $60 \mathrm{~min}$ ) and TOS1 (at $60 \mathrm{~min}$ ) were down-regulated in the hat1 $\triangle$, hat2 $\triangle$ double mutant. HWP1, PGA54, PHR1, TOS1, PGA6 and HYR1 have been found to be up-regulated in previous studies in our laboratory on transcriptional response of $C$. albicans to temperature (Rashki, Ph.D thesis 2009).

Table 2.4. Differentially expressed genes in hat $2 \Delta$ mutant at the three times of incubation (at 15,60 and $180 \mathrm{~min})$

\begin{tabular}{|c|c|c|c|c|c|}
\hline Gene name & ORF & Function & \multicolumn{3}{|c|}{ Fold regulation } \\
\hline \multicolumn{6}{|c|}{ Up-regulated genes } \\
\hline Glycolysis & & & $15 \mathrm{~min}$ & $60 \mathrm{~min}$ & $180 \mathrm{~min}$ \\
\hline $\mathrm{ADH} 1$ & orf19.3997 & Alcohol dehydrogenase & 5.91 & 1.37 & 0.96 \\
\hline ADH5 & orf19.2608 & Putative alcohol dehydrogenase & 1.76 & 0.79 & 0.72 \\
\hline ALD5 & orf19.13228 & An aldehyde dehydrogenase & 0.81 & 0.94 & 1.79 \\
\hline CDC19 & orf19.3575 & Putative pyruvate kinase & 3.66 & 0.81 & 1.93 \\
\hline ENO1 & orf19.395 & $\begin{array}{l}\text { Enolase (2-phospho-D-glycerate- } \\
\text { hydrolyase) }\end{array}$ & 4.77 & 0.81 & 1.26 \\
\hline FAB1 & orf19.9088 & $\begin{array}{l}\text { Phosphatidylinositol 3-phosphate 5- } \\
\text { kinase }\end{array}$ & 1.15 & 1.24 & 1.70 \\
\hline FBA1 & orf19.4618 & Putative fructose-bisphosphate aldolase & 2.97 & 0.64 & 1.01 \\
\hline GPH1 & orf19.7021 & Putative glycogen phosphorylase & 2.25 & 0.74 & 0.93 \\
\hline GPM1 & orf19.903 & Described as phosphoglycerate mutase & 2.54 & 0.55 & 1.08 \\
\hline GLG2 & orf19.7434 & Protein described as self-glucosylating & 1.75 & 1.28 & 1.42 \\
\hline HXK2 & orf19.8176 & Protein described as hexokinase II & 2.66 & 0.93 & 0.79 \\
\hline PCK1 & orf19.7514 & Phosphoenolpyruvate carboxykinase & 3.68 & 0.90 & 1.04 \\
\hline PDC11 & orf19.2877 & $\begin{array}{l}\text { Protein similar to pyruvate } \\
\text { decarboxylase }\end{array}$, & 2.62 & 0.92 & 1.19 \\
\hline
\end{tabular}




\begin{tabular}{|c|c|c|c|c|c|}
\hline PFK2 & orf19.6540 & Beta subunit of phosphofructokinase & 1.88 & 0.61 & 1.07 \\
\hline PGK1 & orf19.3651 & Phosphoglycerate kinase & 3.93 & 1.03 & 1.07 \\
\hline TPI1 & orf19.6745 & Putative ortholog of $S$. cerevisiae Tpi1p & 1.58 & 0.93 & 0.85 \\
\hline TKL4 & orf19.5112 & Putative transketolase & 1.08 & 0.39 & 1.78 \\
\hline \multicolumn{6}{|l|}{ Cell wall } \\
\hline BMT5 & orf19.1464 & Putative beta-mannosyltransferase & 3.30 & 1.48 & 1.15 \\
\hline ВMT9 & orf19.4673 & Putative beta-mannosyltransferase & 1.64 & 2.02 & 0.98 \\
\hline CHS2 & orf19.7298 & Chitin synthase & 1.99 & 2.55 & 0.84 \\
\hline GSL1 & orf19.2495 & Subunit of beta-1.3-glucan synthase & 1.77 & 2.58 & 1.08 \\
\hline IRS4 & orf19.6953 & Protein with roles in cell wall integrity & 1.14 & 1.67 & 0.99 \\
\hline FGR41 & orf19.4910 & Putative GPI-anchored protein & 1.35 & 2.18 & 1.20 \\
\hline HWP1 & orf19.1321 & Hyphal cell wall protein & 0.76 & 1.05 & 1.78 \\
\hline PGA45 & orf19.2451 & Cell wall protein; putative GPI-anchor & 2.08 & 1.01 & 2.24 \\
\hline PGA54 & orf19.2685 & Putative GPI-anchored protein & 0.59 & 0.61 & 1.89 \\
\hline \multicolumn{6}{|c|}{ Glucose transport } \\
\hline HGT1 & orf19.4527 & High-affinity glucose transporter & 1.81 & 0.54 & 1.69 \\
\hline HGT7 & orf19.2023 & Putative glucose transporter & 2.29 & 1.47 & 1.97 \\
\hline HGT8 & orf19.2021 & Putative glucose transporter & 2.14 & 1.33 & 1.44 \\
\hline HGT9 & orf19.644 & Putative glucose transporter & 1.43 & 2.75 & 0.98 \\
\hline SHA3 & orf19.3669 & $\begin{array}{l}\text { Protein similar to } \mathrm{S} \text {. cerevisiae Sha3p, } \\
\text { which is a serine/threonine kinase } \\
\text { involved in glucose transporter }\end{array}$ & 1.92 & 2.09 & 1.42 \\
\hline \multicolumn{6}{|c|}{ Transcription factors } \\
\hline CTA29 & orf19.7127.1 & $\begin{array}{l}\text { Transcription is upregulated in an RHE } \\
\text { mode }\end{array}$ & 1.29 & 1.38 & 1.98 \\
\hline IPF19804 & orf19.3835 & Putative DNA-binding transcription factor & 1.93 & 1.40 & 1.06 \\
\hline GAL4 & orf19.5338 & $\begin{array}{l}\text { Putative transcription factor with zinc } \\
\text { cluster DNA-binding motif }\end{array}$ & 1.14 & 0.90 & 2.48 \\
\hline MRR1 & orf19.7372 & Regulator of MDR1 transcription & 1.31 & 8.69 & 1.00 \\
\hline TYE7 & orf19.4941 & Putative bHLH transcription factor & 2.22 & 1.46 & 1.73 \\
\hline \multicolumn{6}{|c|}{ Telomere-proximal genes family } \\
\hline TLO3 (CTA2) & orf19.6112 & $\begin{array}{l}\text { Transcriptional activator family of } \\
\text { telomere-proximal genes }\end{array}$ & 1.17 & 1.18 & 1.72 \\
\hline TLO4 & orf19.7276.1 & $\begin{array}{l}\text { Member of a family of telomere-proximal } \\
\text { genes }\end{array}$ & 1.25 & 1.38 & 1.68 \\
\hline TLO12 (CTA24) & orf19.4054 & $\begin{array}{l}\text { Transcriptional activator family of } \\
\text { telomere-proximal genes }\end{array}$ & 1.11 & 0.85 & 1.75 \\
\hline \multicolumn{6}{|c|}{ Ribosomal protein } \\
\hline RPL4B & orf19.7217 & Putative ribosomal protein & 1.72 & 1.18 & 1.11 \\
\hline RPL5 & orf19.6541 & Predicted ribosomal protein & 1.75 & 1.44 & 1.26 \\
\hline RPL33 & orf19.6882.1 & Ribosomal protein L35a & 0.98 & 1.78 & 1.12 \\
\hline RPL42 & orf19.4909.1 & Ribosomal protein L36a & 1.77 & 1.20 & 1.07 \\
\hline RPS6A & orf19.4660 & Predicted ribosomal protein & 1.11 & 2.15 & 1.16 \\
\hline \multicolumn{6}{|c|}{$\begin{array}{l}\text { Down-regulated genes } \\
\text { Respiration }\end{array}$} \\
\hline $\mathrm{COB}$ & & Cytochrome b & 0.41 & 0.88 & 1.12 \\
\hline cox1 & & Subunit I of cytochrome c oxidase & 0.59 & 0.73 & 1.43 \\
\hline COX3A & & Subunit III of cytochrome c oxidase & 0.41 & 0.70 & 1.28 \\
\hline COX3B & & Subunit III of cytochrome c oxidase & 0.48 & 0.74 & 1.49 \\
\hline coX6 & orf19.873.1 & Flucytosine induced & 0.88 & 0.47 & 1.33 \\
\hline COX15 & orf19.3656 & $\begin{array}{l}\text { Transcription is regulated by Nrg1p and } \\
\text { Tup1p }\end{array}$ & 0.79 & 0.64 & 1.11 \\
\hline NAD2 & & $\begin{array}{l}\text { Subunit } 2 \text { of NADH: ubiquinone } \\
\text { oxidoreductase }\end{array}$ & 0.41 & 0.69 & 1.74 \\
\hline NAD6 & & $\begin{array}{l}\text { Subunit } 6 \text { of NADH: ubiquinone } \\
\text { oxidoreductase }\end{array}$ & 0.48 & 0.81 & 1.20 \\
\hline \multicolumn{6}{|l|}{ Cell wall } \\
\hline ALS4 & orf19.4556 & ALS family protein & 0.95 & 0.55 & 0.78 \\
\hline ECM31 & orf19.6057 & $\begin{array}{l}\text { Involved in cell wall biogenesis and } \\
\text { architecture }\end{array}$ & 0.93 & 0.52 & 0.74 \\
\hline GSC1 & orf19.2929 & Subunit of beta-1.3-glucan synthase & 0.50 & 0.32 & 0.97 \\
\hline HYR1 & orf19.12440 & Nonessential, GPI anchored & 1.06 & 0.71 & 0.51 \\
\hline HYR3 & orf19.575 & $\begin{array}{l}\text { Putative GPI-anchored protein of } \\
\text { unknown function }\end{array}$ & 0.94 & 0.48 & 1.02 \\
\hline PHR2 & orf19.3829 & Glycosidase of cell surface & 0.50 & 0.67 & 1.44 \\
\hline PGA6 & orf19.12229 & $\begin{array}{l}\text { Putative GPI-anchored cell-wall protein } \\
\text { of unknown function }\end{array}$ & 0.85 & 0.51 & 1.25 \\
\hline PGA63 & orf19.6217 & Putative protein of unknown function & 1.05 & 0.34 & No data \\
\hline TOS1 & orf19.1690 & $\begin{array}{l}\text { Similar to alpha agglutinin anchor } \\
\text { subunit }\end{array}$ & 0.59 & 0.81 & 1.24 \\
\hline
\end{tabular}




\begin{tabular}{|c|c|c|c|c|c|}
\hline \multicolumn{6}{|c|}{ Ribosomal protein } \\
\hline RPL11 & orf19.2232 & Predicted ribosomal protein & 0.49 & 1.01 & 0.86 \\
\hline RPL20B & orf19.4632 & Predicted ribosomal protein & 0.58 & 0.77 & 0.84 \\
\hline RPL8B & orf19.6002 & Predicted ribosomal protein & 0.51 & 0.84 & 1.00 \\
\hline RPS4A & orf19.5341 & Predicted ribosomal protein & 0.57 & 0.67 & 0.78 \\
\hline RPS15 & orf19.5927 & Putative ribosomal protein & 0.49 & 0.69 & 1.10 \\
\hline MRPL7 & orf19.2214 & Ribosomal protein of the large subunit & 0.96 & 0.47 & 1.11 \\
\hline MRPL19 & orf19.13611 & Protein described as a ribosomal protein & 1.00 & 0.47 & 0.98 \\
\hline MRPL38 & orf19.5684 & $\begin{array}{l}\text { Ribosomal protein of the large } \\
\text { subunit(L14) }\end{array}$ & 0.69 & 0.54 & 1.23 \\
\hline RPL10 & orf19.10452 & Putative ribosomal protein & 0.68 & 0.54 & 1.29 \\
\hline RPL14B & orf19.4931.1 & Ribosomal protein L14B & 0.89 & 0.39 & 0.98 \\
\hline RPL16A & orf19.6085 & $\begin{array}{l}\text { Protein similar to } S \text {. cerevisiae ribosomal } \\
\text { protein Rpl16Ap }\end{array}$ & 0.70 & 0.38 & 0.90 \\
\hline RPS16 & orf19.2994.1 & Ribosomal protein & 0.101 & 0.49 & 0.89 \\
\hline RPL82 & orf19.2311 & Predicted ribosomal protein & 0.46 & 0.51 & 0.97 \\
\hline \multicolumn{6}{|c|}{ Transcription factors } \\
\hline $\mathrm{CPH} 1$ & orf19.4433 & $\begin{array}{l}\text { Transcription factor required for mating } \\
\text { and hyphal growth }\end{array}$ & 0.71 & 0.40 & 1.26 \\
\hline CAP1 & orf19.9191 & Transcription factor & 0.85 & 0.47 & 1.15 \\
\hline CTA4 & orf19.7374 & Predicted transcription factor & 0.85 & 0.56 & 0.85 \\
\hline CTA8 & orf19.4775 & Protein that activates transcription & 0.81 & 0.60 & 1.19 \\
\hline HAC1 & orf19.2432 & Putative transcription factor & 0.56 & 0.88 & 1.26 \\
\hline MIG1 & orf19.4318 & Transcriptional repressor & 0.85 & 0.38 & 0.95 \\
\hline SPT20 & orf19.422 & $\begin{array}{l}\text { Protein described as a transcription } \\
\text { factor }\end{array}$ & 0.62 & 1.36 & 1.49 \\
\hline TAF145 & orf19.735 & $\begin{array}{l}\text { Putative DNA-binding transcription } \\
\text { factor; putative TFIID subunit; } \\
\text { flucytosine repressed }\end{array}$ & 0.65 & 0.89 & 1.33 \\
\hline TBP1 & orf19.1837 & Transcription initiation factor & 0.79 & 0.38 & 1.15 \\
\hline TUP1 & orf19.6109 & Transcriptional corepressor & 1.25 & 0.55 & 1.43 \\
\hline ZCF20 & orf19.11621 & $\begin{array}{l}\text { Predicted zinc-finger protein of unknown } \\
\text { function }\end{array}$ & 0.56 & 1.28 & 0.69 \\
\hline ZPR1 & orf19.3300 & Protein with putative zinc finger & 0.57 & 0.59 & 1.07 \\
\hline \multicolumn{6}{|c|}{ Ergoestrol biosynthesis } \\
\hline ERG4 & orf19.5379 & As similar to sterol C-24 reductase & 1.13 & 0.43 & 1.02 \\
\hline ERG7 & orf19.9143 & 2,3-epoxysqualene-lanosterol cyclise & 0.89 & 0.42 & 1.09 \\
\hline ERG9 & orf19.3616 & $\begin{array}{l}\text { Putative farnesyl-diphosphate farnesyl } \\
\text { transferase }\end{array}$ & 1.23 & 0.42 & 0.96 \\
\hline ERG26 & orf19.2909 & C-3 sterol dehydrogenase & 1.03 & 0.58 & 0.77 \\
\hline
\end{tabular}

Genes involved in protein synthesis and ribosomal proteins

A second important category includes up-regulation of $R P L 42, R P L 4 B, R P L 5$ (at 15 min), RPS6A, RPL33 (at $60 \mathrm{~min}$ ) and down-regulation of RPL11, RPL20B, RPL8B, RPS15, RPS4A (at $15 \mathrm{~min}$ ), MRPL7, MRPL19, MRPL38, RPL10, RPL14B, RPL16A, RPS16 (at $60 \mathrm{~min}$ ) and RPL82 (at 15 and $60 \mathrm{~min}$ ) in the hat2 $\triangle$ mutant. Two genes, RPL33 and RPS4A, that have been found to be up-regulated in previous studies on transcriptional response of C. albicans to temperature (Rashki, Ph.D thesis 2009), were up- and down-regulated in our experiment respectively. No genes of this category have been found to be up- and down-regulated in the hat1 $\Delta$ mutant.

\section{Central carbon metabolism}

The regulation of 17 genes (13 up-regulated after $15 \mathrm{~min}$ ) involved in the glycolytic pathway was detected. The expression all of them decreased with time, suggesting a 
variation in regulation of this group of genes in hat $2 \Delta$ mutant during the yeast-hypha transition (Table 2.4). Only expression three genes, ADH1, ENO1 and PDC11 have been found to be up-regulated in the hat1 $\Delta$ mutant after 15 min of incubation. The expression all of them have been reported to be down-regulated after $15 \mathrm{~min}$ and increased with time in the transcriptional response of $C$. albicans to temperature (Rashki, Ph.D thesis 2009) suggesting that HAT2 play a role in regulation of this set of genes.

Expression all of this group of genes were down-regulated in the hat $1 \Delta$, hat $2 \Delta$ double mutant and have been reported to be decreased in the gcn5 $\Delta$ and gcn5 $\Delta$, hda $1 \Delta$ and odc1 $1 \Delta$ mutant strains during the yeast-hypha transition (Rashki, Ph.D thesis 2009). These changes reflect differences in the modulation of metabolic genes during the hypha transition in different mutant strains. We have found up-regulation of five genes belonging to the glucose transporter HGT1, HGT7 (at 15 and $180 \mathrm{~min}$ ), HGT8 (at 15 min), HGT9 (at $60 \mathrm{~min}$ ) and SHA3 (at 15 and $60 \mathrm{~min}$ ) (Brown et al., 2006; Davis et al., 2002; Fan et al., 2002; Uhl et al., 2003; Varma et al., 2000) in the hat2 $\Delta$ mutant.

\section{Set of genes regulated only at one incubation time}

After 15 and 60 minutes of incubation at $37^{\circ} \mathrm{C}$, several sets of genes were either up- or down-regulated. The main groups of up-regulated genes at one time point included five genes contributing to stress response, HSP12 (at $15 \mathrm{~min}$ ), HSP70, HSP90, SSA2 and SSC1 (at $180 \mathrm{~min}$ ) (Supplemenary table 2.3). Among the up-regulated genes after 15 min of incubation were HAT1 (histone acetyltransferase), PLB3 (putative secreted phospholipase B), two genes encode for DRAP deaminase (RIB2 and RIB21), RIM21 (protein involved in the $\mathrm{pH}$ response pathway), SMF11 (manganese transporter) and two genes involved in ubiquitin (UB/4 and UBP1) (Supplemenary table 2.3).

The genes up-regulated at $60 \mathrm{~min}$ were $C D C 23$ (similar to anaphase-promoting complex component), CDC73 (protein similar to $S$. cerevisiae Cdc73p), HHT21 (putative histone H3), HOG1 (MAP kinase of osmotic and core stress response) (Supplemenary table 2.3). Among the down-regulated genes after $15 \mathrm{~min}$, we noted six genes corresponding to respiration (COB, COX1, COX $3 A, C O X 3 B, N A D 2$ and NAD6), two genes involved in RNA helicases (DBP2 and DBP9) and four genes involved in transport (DAL9, DUR32, TPO4 and FET34). Among the down-regulated genes after $60 \mathrm{~min}$, we noted four genes corresponding to ergosterol biosynthesis (ERG4, ERG7, ERG9 and ERG26), five genes involved in respiration (ABC1, COX15, COX6, MCI4 and MCR1) and five genes encoding for translation elongation factor (EFB1, EFT2, SUP35, TIF35 and TUF1) (Supplemenary table 2.4). 
A transcriptional profiling analysis was performed to further examine the roles of Hat $1 p$ and Hat2p proteins in $C$. albicans. The transcriptome of the hat1 $\Delta$, hat2 $\Delta$ double mutant was compared with the CAI4 strain and genes that displayed reproductive and statistically significant changes in expression were identified under both yeast growth conditions and during the yeast-hypha switch. A list of differentially expressed genes in the double mutant is provided as supplementary data (Supplementary tables 2.5-2.8). To explore the functions of the hat $1 \Delta$, hat $2 \Delta$ double mutant under conditions that promoted growth in the yeast form, we compared the transcriptomes of CAI4 and the double mutant strain in YNB medium at $28^{\circ} \mathrm{C}$. In the hat $1 \Delta$, hat $2 \Delta$ double mutant we detected an altered expression of 219 genes by $\geq 1.5$-fold: however in the transcriptome of cells growing in Lee medium at $37^{\circ} \mathrm{C}$ during the hypha transition, 793 genes modified their expression level, including 376 up-regulated genes and 417 down-regulated genes. Approximately $16 \%$ of the $C$. albicans genes showed significant changes in their expression levels $(\rho$-value $\leq 0.02)$, indicating a global transcriptional response.

\section{Differential gene expression in exponential growth phase}

During the yeast mode of growth more genes were up-regulated (160 genes) than down-regulated (59 genes). For up-regulated genes, the transcript ratios had values $\geq 1.5-2.0$ for 113 genes, $2-3$ for 37 genes (e. g. SMF11, PTR2) and $\geq 3$ for ten genes (e.g. IPF8762, IPF525, HSP12 and CCL1). For down-regulated genes, the transcript ratios had values between $0.33-0.66$ for 51 genes and $<0.33$ for eight genes.

Distinct groups of genes were either up- or down-regulated during the yeast mode of growth in the hat1 $\Delta$, hat2 $\Delta$ double mutant (Table 2.5).

Table 2.5 Differentially expressed genes in hat $1 \Delta$, hat $2 \Delta$ mutant during the yeast mode of growth

\begin{tabular}{|c|c|c|c|}
\hline Gene name & ORF & Function & Fold regulation \\
\hline \multicolumn{4}{|c|}{$\begin{array}{l}\text { Up-regulated genes } \\
\text { Cell wall }\end{array}$} \\
\hline BMT6 & orf19.5602 & Putative beta-mannosyltransferase & 1.61 \\
\hline CHS1 & orf19.4477 & Member of aldo-ketonreductase family & 2.22 \\
\hline CPS37 & orf19.2531 & Plasma membrane & 3.14 \\
\hline IFF11 & orf19.5399 & Secreted protein required for normal cell wall structure & 2.67 \\
\hline IPF885 & orf19.7214 & $\begin{array}{l}\text { Protein described as similar to glucan 1.3-beta- } \\
\text { glucosidase }\end{array}$ & 1.99 \\
\hline PGA10 & orf19.5674 & $\begin{array}{l}\text { Plasma membrane protein involved in heme-iron } \\
\text { utilization }\end{array}$ & 1.92 \\
\hline PGA14 & orf19.968 & Putative GPI-anchored protein of unknown function & 1.69 \\
\hline PIR1 & orf19.220 & Structural protein of cell wall & 1.57 \\
\hline RBT5 & orf19.5636 & GPI-anchored cell wall protein & 2.12 \\
\hline RHD3 & orf19.5305 & $\begin{array}{l}\text { Putative GPI-anchored protein that localizes to the cell } \\
\text { wall }\end{array}$ & 1.61 \\
\hline
\end{tabular}




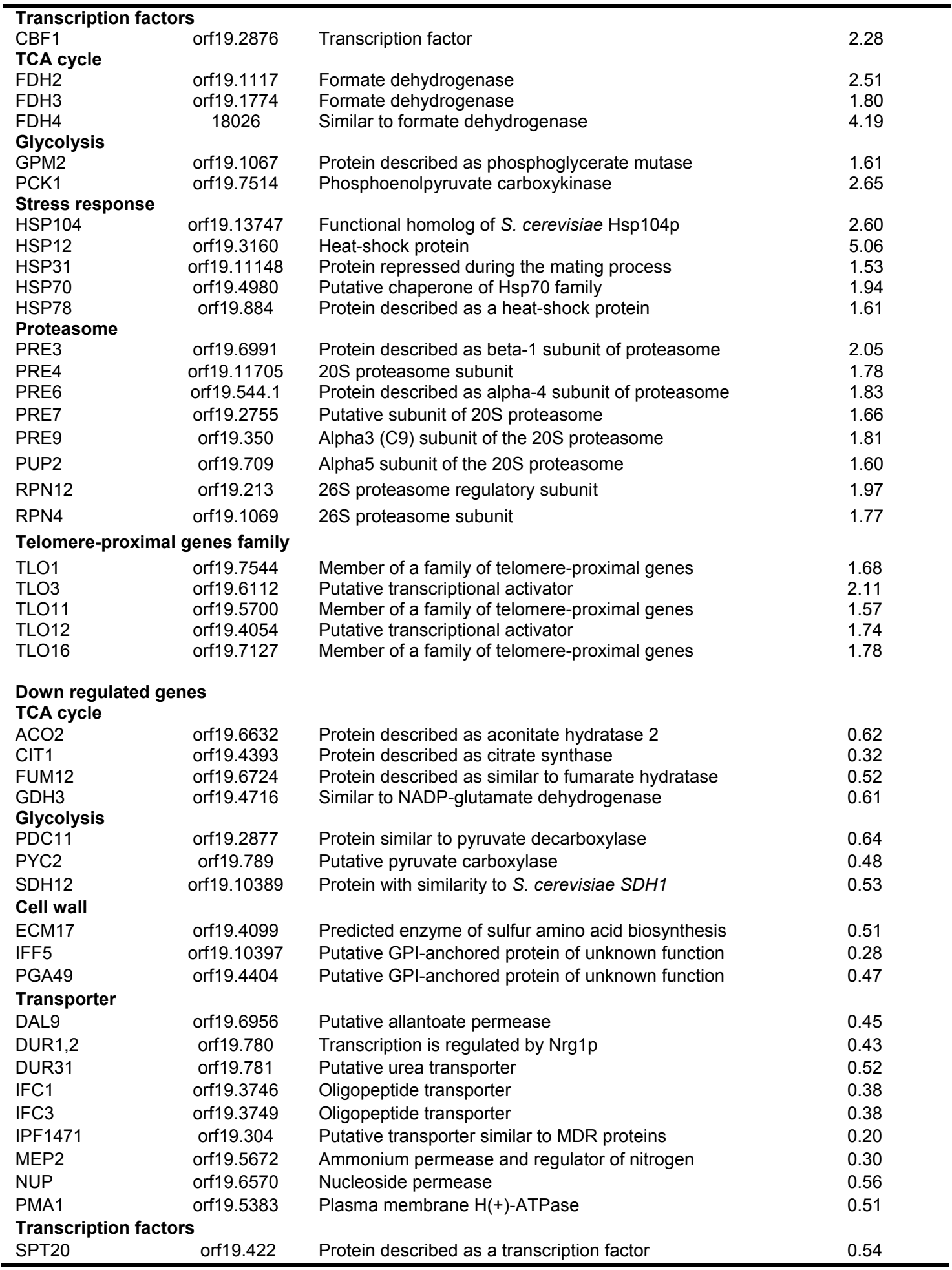

We have found up-regulated in the hat1 (TLO2, TLO3, TLO4, TLO9, TLO10, TLO11, TLO12 and TLO34) and in the hat1 $\triangle$, hat2 $\triangle$ mutants a family of genes involved in telomeric silencing although those genes have not been described in other organisms (TLO1, TLO3, TLO11, TLO12, TLO16) (Harcus et al., 2004; Maglott et al., 2007; van het Hoog et al., 2007). 
We proposed that in C. albicans the HAT1 gene plays a role in telomeric silencing. In Saccharomyces cerevisiae has been reported that deletion of the HAT1 and/or HAT2 gene doesn't changes in the level of telomeric silencing (Poveda et al., 2004). This result is a difference between the putative Hat-B complex of $C$. albicans and $S$. cerevisiae.

However, in the double mutant, we detected the up-regulation of ten genes encoding for cell wall proteins (IFF11) (Bates et al., 2007), BMT6, CHS1, CSP37, RBT5, RHD3, IPF885, PGA10, PGA14 and PIR1) and down-regulation of three cell wall genes (ECM17, IFF5 and PGA49), of five genes involved in stress response (HSP104, HSP12, HSP31, HSP70 and HSP78), of eight genes encoding for proteasome (PRE3, PRE4, PRE6, PRE7, PRE9, PUP2, RPN12 and RPN4).

Our results were in contrast which those obtained with the hat1 $\Delta$ simple mutant, in which genes encoding for proteasome and cell wall proteins and the majority of genes involved in the glycolytic pathway and sugar metabolism did not appear regulated during the yeast mode of growth. We can conclude that the transcriptional response in C. albicans hat $1 \Delta$, hat $2 \Delta$ double mutant differs significantly from those of hat $1 \Delta$ and hat $2 \Delta$ simple mutants.

\section{Transcriptional profile of the hat $1 \Delta$, hat $2 \Delta$ during the yeast-hypha transition}

Next, we focused on the identification of genes whose expression was altered in the double mutant strain during the yeast-hypha transition. We generated the transcriptional profiles for the hat $1 \Delta$, hat $2 \Delta$ mutant in Lee medium for 15,60 and 180 min at $37^{\circ} \mathrm{C}$ using the CAl4 strain as reference. A total of 793 genes were found to change at the consensus level of $\geq 1.5$ fold, including 376 up- and 417 down-regulated genes. The distribution of genes altered at the three points is represented in the Venn diagram, and the clustering of genes regulated by deletion of HAT1 and HAT2 is represented in Fig. 2.17 ( $A$ and $B$ ).

We found that 16 genes were common to the three times assayed ( 6 genes up- and 10 down-regulated), 193 genes at 15 and 60 min (62 up- and 131 down-) and 16 genes at 60 and 180 min (10 up- and 6 down-). 272 genes (189 up- and 83 down-), 265 genes (97 up- and 168 down-) and 29 genes (10 up- and 19 down-) varied only at one time of induction (at 15, 60 and 180 min respectively). However, only 2 genes were common between 15 and 180 min validating our experimental approach. Sixteen genes common at the three times are listed in Table 2.6. 


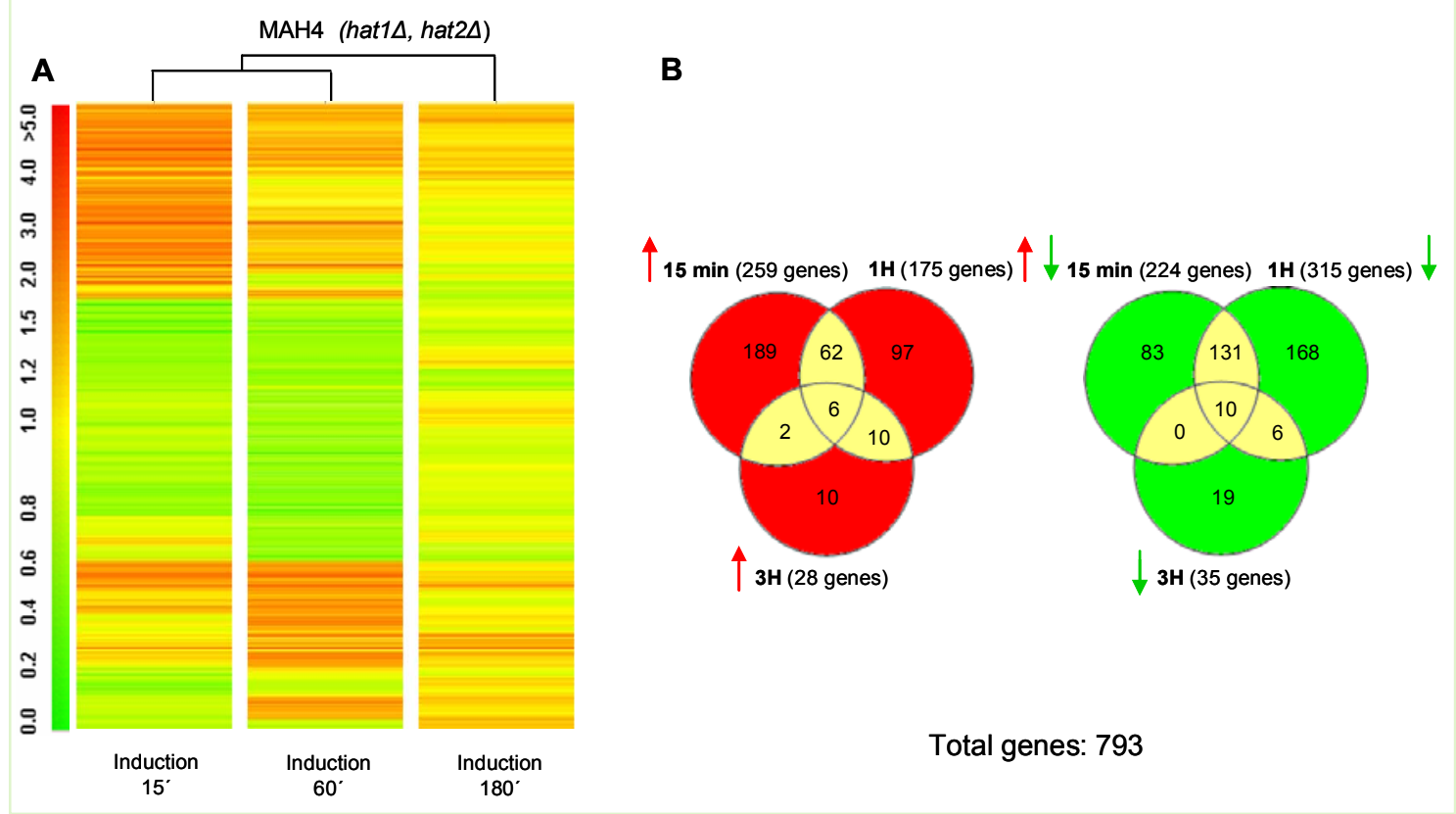

Figure 2.17 Transcript profiles of $C$. albicans hat1 $\Delta$, hat2 $\Delta$ double mutant during the yeast-hypha transition (A). Venn diagrams of genes regulated by Hat1p and Hat2p in the double mutant (B). The CAI4 strain was used as reference, which all were grown in identical conditions: Lee $/ 37^{\circ} \mathrm{C}$ (inducing).

Table 2.6 Common differentially expressed genes in hat $1 \Delta$, hat $2 \Delta$ mutant at the three times of incubation (at 15,60 and $180 \mathrm{~min}$ )

\begin{tabular}{|c|c|c|c|c|c|}
\hline $\begin{array}{l}\text { Gene } \\
\text { name }\end{array}$ & ORF & Description & $\begin{array}{c}\text { Fold } \\
\text { regulation } \\
15 \text { min }\end{array}$ & $\begin{array}{c}\text { Fold } \\
\text { regulation } \\
60 \mathrm{~min}\end{array}$ & $\begin{array}{c}\text { Fold } \\
\text { regulation } \\
180 \mathrm{~min}\end{array}$ \\
\hline \multicolumn{6}{|c|}{ Up-regulated genes } \\
\hline COX3A & & Subunit III of cytochrome c oxidase & 1.85 & 2.38 & 1.97 \\
\hline COX3B & & Subunit III of cytochrome c oxidase, & 1.63 & 1.88 & 1.78 \\
\hline CTR1 & orf19.3646 & Copper transporter & 2.01 & 3.86 & 2.09 \\
\hline HHT1 & orf19.6791 & Putative histone $\mathrm{H} 3$ & 2.37 & 2.21 & 2.36 \\
\hline RPL2 & orf19.2309.2 & Ribosomal protein L8 & 2.78 & 3.32 & 2.34 \\
\hline RPL27A & orf19.5225.2 & Ribosomal protein L27 & 1.76 & 1.79 & 1.68 \\
\hline \multicolumn{6}{|c|}{ Down-regulated genes } \\
\hline $\mathrm{ADH} 1$ & orf19.3997 & Alcohol dehydrogenase & 0.39 & 0.38 & 0.59 \\
\hline ATP14 & orf19.5491.1 & Macrophage/pseudohyphal-induced & 0.59 & 0.43 & 0.49 \\
\hline ATP4 & orf19.3579 & Macrophage/pseudohyphal-induced & 0.40 & 0.36 & 0.58 \\
\hline CDC19 & orf19.3575 & Putative pyruvate kinase & 0.20 & 0.34 & 0.49 \\
\hline ERG251 & orf19.12101 & $\begin{array}{l}\text { Ketoconazole-induced; amphotericin B, } \\
\text { caspofungin repressed }\end{array}$ & 0.44 & 0.36 & 0.51 \\
\hline FCY24 & orf19.7331 & Putative transporter & 0.49 & 0.39 & 0.57 \\
\hline PDC11 & orf19.2877 & Protein similar to pyruvate decarboxylase; & 0.29 & 0.37 & 0.63 \\
\hline PHM7 & orf19.2170 & Putative transporter & 0.60 & 0.41 & 0.64 \\
\hline YHB1 & orf19.3707 & Nitric oxide dioxygenase & 0.46 & 0.59 & 0.45 \\
\hline YTH1 & orf19.6881 & $\begin{array}{l}\text { Protein described as an mRNA cleavage } \\
\text { and polyadenylation }\end{array}$ & 0.26 & 0.29 & 0.42 \\
\hline
\end{tabular}


Six up-ORFs can be classified in respiration (i.e $C O X 3 A$ and $C O X 3 B$ ), one gene encodes for copper transporter (CTR1), two genes encode for ribosomal proteins (RPL2 and RPL27A) and one gene is involved in histone assembly (HHT1). The ten commonly down-regulated genes belong to the glycolytic pathway were $A D H 1, C D C 19$ and PDC11, two genes involved in ATP biosynthesis (ATP14 and ATP4), two genes encoding for putative transporter proteins (FCY24 and PHM7) and three genes with different function included ERG251 (putative C-4 methyl sterol oxidase) (Marichal et al., 1999), YHB1 (nitric oxide dioxygenase) and YTH1 (essential RNA-binding component of cleavage and polyadenylation factor) (Barabino et al., 2000).

\section{Common up- and down-regulated genes after two time points of induction}

The analysis of the microarray data of cells obtained during the hyphal transition showed a total of 193 genes to be differentially expressed commonly between 15 and 60 min of induction: 62 genes were up-regulated and 131 were down-regulated (Supplementary tables 2.7 and 2.8).

Among the up-regulated genes, we highlight the induction of genes encoding for rRNA processing protein (RPL11, RPL14B, RPL23A, RPS24, RRP1, RSA2, SIK1 and SNU23), genes encoding for translation initiation factors (SUI2, TEF1 and TIF11), genes encoding for stress-response proteins (HSP70 and HSP9O) and histone assembly (HHF1 and HHF22). Among the down-regulated genes, we observed genes encoding for cell wall proteins (ALS1, ALS3, ALS4, ECM21, FGR51, HYR1, IPF8796, PGA63, PMT1, PMT2, PMT4 and RBT1), glycolytic pathway (ADH2, PFK1, PFK2, GPM1, GSY1, PGK1 and TPS2), glucose transport (HGT6, HGT7 and HGT8), cell polarity and signal transduction (ARP3, ARP8, BEM1, BEM2, BUD2, CLC1, MYO2, RAS1, RGS2 and TPM2), secretory pathway (ERV46, SEC11, SEC16, SEC24, SEC61, SEC9) and transcription factors (ECM22, HAC1, SSN6, TEC1 and UME6). The down-regulation of genes involved in the secretory pathway and in o-glycosylation has been reported to be down-regulated in odc1 $\Delta$ mutant after 15 and 60 min of incubation (Rashki, Ph.D thesis 2009). Differentially expression of the PMT genes family were also reported in pmt mutant strains (Cantero et al., 2007).

Of the total up- and down-regulated genes between 60 and 180 min of incubation, we observed common differential expression of sixteen genes; ten were up-regulated and six genes were down-regulated.

Of the ten ORFs, we detected three genes involved in respiration (COB, NAD2 and $N A D 6$ ), two genes encoding for ferric reductases (FRE7 and FRE30), one gene encoding for a predicted GPI anchor (PGA10), one gene encoding for a predicted 
ribosomal protein (RPS4A), and two genes of unknown function. The down-regulated genes, were CRH11 (putative GPI-anchor), CYT1 (component of the mitochondrial respiratory chain), IPF11725 (described as a Gag-related protein), IPF3912 (similar to $S$. cerevisiae TOS8 a putative transcription factor found associated with chromatin), QCR8 (Subunit 8 of ubiquinol cytochrome-c reductase complex) and ZRT2 (a protein described as predicted zinc transporter).

\section{Sets of genes regulated only at one incubation time}

A total of 272 genes were expressed differentially at 15 min of hypha transition, among which 189 and 83 genes were up- and down-regulated respectively.

Expression of some genes include those encoding for rRNA processing protein (MPP10, MRP1, MRP7, MRPL16, MRPL17, MRPL31, MRPL36, RPF1, RPL10, RPL21A, RPL25, RPL37B, RPL39, RPL40B, RPL6, RPL9B, RPS1, RPS17B, RPS19A, RPS25B, RPS28B, RPS3, RPS7A), genes encoding for nucleolar proteins (MAK16, MAK21, MAK5, NOP1, NOP14, NOP15, NOP2, NOP4, NOP5, RRP15, RRP6, RRP8), genes involved in DNA-direcred RNA polymerase (RPA190, RPA43, RPA49, RPC10, $R P C 25, R P C 40)$, stress response (ASR1, ASR3, DDR48, HSP12, SOD5, SSA2), elongation factors (EFT2, SUI3, TFS1, TIF1, TIF3) and one transcription factor (ZPR1) (Rogers \& Barker, 2003) were up-regulated. The main categories of down-regulated genes were correspond to those encoding for cell wall proteins (ALS2, EAP1, ECE1, ECM17, ECM33, HWP1, MNT1, PGA54, PGA58, PGA62), secretory pathway (CHC1, ERV29, SEC23, SEC26), glycolytic pathway and glyoxylate cycle (ENO1, GLK1, ICL1, MIS11, MLS1, TDH3, TPI1) and transcription factors (GAL4, IPF29, SKO1) (Table 2.7).

Table 2.7 Differentially expressed genes in hat $1 \Delta$, hat $2 \Delta$ mutant during the yeast-hypha transition (at 15 , 60 and $180 \mathrm{~min}$ )

\begin{tabular}{|c|c|c|c|c|c|}
\hline Gene name & ORF & \multirow[t]{2}{*}{ Function } & \multicolumn{3}{|c|}{ Fold regulation } \\
\hline \multicolumn{5}{|c|}{ Up-regulated genes } & \\
\hline \multicolumn{3}{|c|}{ Amino acid biosynthesis } & $15 \mathrm{~min}$ & $60 \mathrm{~min}$ & $180 \mathrm{~min}$ \\
\hline ARG1 & orf19.7469 & Similar to argininosuccinate synthase & 1.37 & 1.89 & 0.81 \\
\hline ARG3 & orf19.5610 & Alkaline downregulated & 1.36 & 2.63 & 0.82 \\
\hline GAP4 & orf19.4456 & Putative amino acid permease & 1.21 & 1.72 & 1.21 \\
\hline MET14 & orf19.946 & Predicted role in sulfur metabolism & 1.14 & 1.53 & 1.43 \\
\hline MET3 & orf19.5025 & $\begin{array}{l}\text { Putative ATP sulfurlyase of sulfate } \\
\text { assimilation }\end{array}$ & 1.22 & 1.61 & 1.32 \\
\hline SAM2 & orf19.657 & S-adenosylmethionine synthetase & 1.04 & 1.98 & 1.15 \\
\hline \multicolumn{6}{|c|}{ Stress response } \\
\hline ASR1 & orf19.2344 & $\begin{array}{l}\text { Protein described as similar to heat shock } \\
\text { proteins }\end{array}$ & 1.72 & 1.15 & 1.07 \\
\hline ASR3 & orf19.8462 & $\begin{array}{l}\text { Gene regulated by cAMP and by osmotic } \\
\text { stress }\end{array}$ & 1.65 & 0.78 & 0.87 \\
\hline HSP60 & orf19.717 & $\begin{array}{l}\text { Protein described as mitochondrial heat } \\
\text { shock protein }\end{array}$ & 1.29 & 2.14 & 0.73 \\
\hline HSP70 & orf19.4980 & Putative chaperone of Hsp70 family & 2.63 & 2.53 & 1.04 \\
\hline HSP90 & orf19.6515 & Chaperone of Hsp90 family & 3.20 & 2.68 & 1.41 \\
\hline MSI3 & orf19.2435 & Protein of the HSP70 family & 2.54 & 2.01 & 1.01 \\
\hline
\end{tabular}




\begin{tabular}{|c|c|c|c|c|c|}
\hline SSA2 & orf19.1065 & Hsp70 family chaperone & 2.19 & 1.49 & 0.76 \\
\hline SSZ1 & orf19.3812 & Protein described as an HSP70 chaperone & 1.60 & 1.06 & 1.01 \\
\hline TSA1 & orf19.7417 & $\begin{array}{l}\text { Protein of TSA/alkyl hydroperoxide } \\
\text { peroxidase C }\end{array}$ & 1.80 & 1.32 & 0.91 \\
\hline \multicolumn{6}{|c|}{ Protein synthesis and ribosomal proteins } \\
\hline MPP10 & orf19.1915 & Downregulated in core stress response & 1.96 & 1.19 & 1.10 \\
\hline MRP1 & orf19.1661.1 & $\begin{array}{l}\text { Mitochondrial ribosomal protein of the small } \\
\text { subunit }\end{array}$ & 1.71 & 1.24 & 0.98 \\
\hline MRP7 & orf19.7203 & $\begin{array}{l}\text { Mitochondrial ribosomal protein YmL2 } \\
\text { precursor }\end{array}$ & 1.78 & 1.35 & 1.02 \\
\hline MRPL16 & orf19.9569 & Ribosomal protein & 1.56 & 1.44 & 1.08 \\
\hline MRPL17 & orf19.585 & Ribosomal protein of the large subunit & 1.74 & 1.14 & 0.91 \\
\hline MRPL31 & orf19.1485 & Mitochondrial ribosomal protein & 1.60 & 1.26 & 1.13 \\
\hline MRPL36 & orf19.3205 & Ribosomal protein YmL36 precursor & 1.89 & 1.48 & 0.84 \\
\hline NMD3 & orf19.706 & Downregulated in core stress response & 1.66 & 1.17 & 1.06 \\
\hline NMD5 & orf19.4188 & Involved in protein-nucleus import & 1.91 & 1.24 & 1.07 \\
\hline PWP1 & orf19.12110 & Downregulated in core stress response & 1.98 & 1.48 & 1.17 \\
\hline RPL10 & orf19.10452 & Ribosomal protein L10 & 1.83 & 1.49 & 1.09 \\
\hline RPL21A & orf19.840 & Putative ribosomal protein & 1.70 & 1.40 & 1.11 \\
\hline RPL25 & orf19.687.1 & Ribosomal protein L23a (by homology) & 1.99 & 1.25 & 1.23 \\
\hline RPL37B & orf19.667.1 & Ribosomal protein & 1.61 & 1.37 & 1.12 \\
\hline RPL39 & orf19.827.1 & Ribosomal protein L39 & 1.73 & 1.16 & 1.24 \\
\hline RPL40B & orf19.4684.2 & $\begin{array}{l}\text { Protein with similarity to the ribosomal } \\
\text { protein portion }\end{array}$ & 1.57 & 1.17 & 1.09 \\
\hline RPL6 & orf19.3003.1 & Protein similar to $S$. cerevisiae Rpl6p & 1.59 & 1.49 & 1.28 \\
\hline RPL9B & orf19.236 & Predicted ribosomal protein & 1.82 & 1.34 & 1.26 \\
\hline RPS1 & orf19.10520 & $\begin{array}{l}\text { Probable ortholog of } S \text {. cerevisiae ribosomal } \\
\text { protein Rp10 }\end{array}$ & 1.78 & 1.28 & 1.42 \\
\hline RPS17B & orf19.2329.1 & Putative ribosomal protein & 1.70 & 1.37 & 1.19 \\
\hline RPS19A & orf19.5996.1 & Putative ribosomal protein & 1.65 & 1.45 & 1.18 \\
\hline RPS25B & orf19.6663 & Predicted ribosomal protein & 1.57 & 1.35 & 1.18 \\
\hline RPS28B & orf19.7048.1 & Ribosomal protein S28B & 1.62 & 1.13 & 1.25 \\
\hline RPS3 & orf19.6312 & Putative ribosomal protein & 1.68 & 1.42 & 1.40 \\
\hline RPS7A & orf19.9267 & Predicted ribosomal protein & 1.71 & 1.49 & 1.26 \\
\hline RRS1 & orf19.6014 & $\begin{array}{l}\text { M utation confers hypersensitivity to } 5 \text { - } \\
\text { fluorocytosine }\end{array}$ & 2.43 & 1.34 & 1.14 \\
\hline SOF1 & orf19.5407 & Involved in $18 \mathrm{~S}$ pre-rRNA production & 1.61 & 1.08 & 1.03 \\
\hline PRP31 & orf19.1296 & Pre-mRNA splicing protein (by homology) & 0.84 & 1.70 & 0.83 \\
\hline RRP43 & orf19.6259 & rRNA processing protein (by homology) & 1.15 & 1.62 & 1.16 \\
\hline RPL20B & orf19.4632 & Predicted ribosomal protein & 1.24 & 1.22 & 1.74 \\
\hline RPL11 & orf19.2232 & Predicted ribosomal protein & 2.47 & 2.06 & 1.29 \\
\hline RPL14B & orf19.4931.1 & Ribosomal protein L14B (by homology) & 2.39 & 2.09 & 1.26 \\
\hline RPL23A & orf19.3504 & Putative ribosomal protein & 3.74 & 1.96 & 1.18 \\
\hline RPS24 & orf19.5466 & Predicted ribosomal protein & 2.14 & 1.80 & 1.08 \\
\hline RRP1 & orf19.6828 & Involved in processing rRNA precursor & 3.30 & 1.81 & 1.12 \\
\hline RSA2 & orf19.6355 & $\begin{array}{l}\text { Involved in ribosome biogenesis (by } \\
\text { homology) }\end{array}$ & 1.77 & 1.60 & 0.97 \\
\hline RPS4A & orf19.5341 & Predicted ribosomal protein & 1.43 & 1.78 & 1.73 \\
\hline RPL2 & orf19.2309.2 & Ribosomal protein L8 & 2.78 & 3.32 & 2.34 \\
\hline $\begin{array}{l}\text { RPL27A } \\
\text { Cell wall }\end{array}$ & orf19.5225.2 & Ribosomal protein L27 & 1.76 & 1.79 & 1.68 \\
\hline CSP37 & orf19.2531 & Plasma membrane, hyphal cell wall protein & 2.17 & 1.27 & 0.93 \\
\hline PGA14 & orf19.968 & $\begin{array}{l}\text { Putative GPI-anchored protein of unknown } \\
\text { function }\end{array}$ & 2.16 & 1.29 & 1.09 \\
\hline PGA52 & orf19.9467 & $\begin{array}{l}\text { Putative GPI-anchored protein of unknown } \\
\text { function }\end{array}$ & 1.74 & 1.05 & 1.06 \\
\hline XOG1 & orf19.2990 & Exo-1,3-beta-glucanase & 1.80 & 0.96 & 0.98 \\
\hline ALG8 & orf19.1659 & Putative glucosyltransferase & 0.86 & 1.67 & 1.14 \\
\hline CSE1 & orf19.1231 & Importin-beta-like protein & 1.26 & 1.55 & 0.83 \\
\hline IFF5 & orf19.10397 & $\begin{array}{l}\text { Putative GPI-anchored protein of unknown } \\
\text { function }\end{array}$ & 1.25 & 1.79 & 1.07 \\
\hline PGA26 & orf19.2475 & $\begin{array}{l}\text { Putative GPI-anchored protein of unknown } \\
\text { function }\end{array}$ & 0.84 & 2.58 & 1.01 \\
\hline PGA59 & orf19.2767 & $\begin{array}{l}\text { Putative GPI-anchored protein of unknown } \\
\text { function }\end{array}$ & 0.86 & 2.04 & 0.94 \\
\hline RBT5 & orf19.5636 & GPI-anchored cell wall protein & 1.26 & 1.45 & 2.16 \\
\hline YWP1 & orf19.3618 & Putative GPI-anchor; cell wall and secreted & 1.02 & 1.38 & 2.23 \\
\hline RBR1 & orf19.535 & $\begin{array}{l}\text { Glycosylphosphatidylinositol anchored cell } \\
\text { wall protein }\end{array}$ & 1.62 & 1.54 & 1.07 \\
\hline PGA10 & orf19.5674 & Predicted GPI anchor & 1.23 & 1.60 & 2.42 \\
\hline \multicolumn{6}{|c|}{ Nucleolar protein } \\
\hline MAK16 & orf19.5500 & $\begin{array}{l}\text { Decreased expression in response to } \\
\text { prostaglandins }\end{array}$ & 1.87 & 1.16 & 0.74 \\
\hline
\end{tabular}




\begin{tabular}{|c|c|c|c|c|c|}
\hline MAK21 & orf19.5912 & $\begin{array}{l}\text { Mutation confers hypersensitivity to } \\
\text { tubercidin }\end{array}$ & 1.71 & 1.45 & 0.97 \\
\hline NOP1 & orf19.3138 & Nucleolar protein; flucytosine induced & 2.32 & 1.13 & 1.11 \\
\hline NOP2 & orf19.501 & $\begin{array}{l}\text { Protein described as; hyphal-induced } \\
\text { expression }\end{array}$ & 2.03 & 1.48 & 1.02 \\
\hline NOP4 & orf19.5198 & Nucleolar protein & 2.27 & 1.36 & 1.27 \\
\hline NOP5 & orf19.1199 & $\begin{array}{l}\text { Protein similar to } S \text {. cerevisiae Nop5p } \\
\text { protein }\end{array}$ & 2.13 & 1.28 & 0.88 \\
\hline NOP14 & orf19.5959 & $\begin{array}{l}\text { Mutation confers resistance to } 5 \text { - } \\
\text { fluorocytosine }\end{array}$ & 1.78 & 1.20 & 0.99 \\
\hline NOP15 & orf19.7050 & A nucleolar ribosome biogenesis factor & 2.23 & 1.40 & 1.14 \\
\hline RRP15 & orf19.563 & $\begin{array}{l}\text { Decreased expression in response to } \\
\text { prostaglandins }\end{array}$ & 1.87 & 1.29 & 1.03 \\
\hline RRP6 & orf19.58 & $\begin{array}{l}\text { Mutation confers hypersensitivity to } 5 \text { - } \\
\text { fluorocytosine }\end{array}$ & 1.55 & 1.27 & 1.02 \\
\hline RRP8 & orf19.3630 & Induced upon biofilm formation & 2.10 & 1.44 & 1.30 \\
\hline \multicolumn{6}{|c|}{ DNA directed RNA polymerase } \\
\hline RPA190 & orf19.1839 & lucytosine induced & 2.05 & 1.16 & 1.02 \\
\hline RPA43 & orf19.2594 & DNA-directed RNA polymerase I & 1.83 & 1.44 & 1.07 \\
\hline RPA49 & orf19.9567 & DNA-directed RNA polymerase A & 2.36 & 1.46 & 1.09 \\
\hline RPC10 & orf19.7255 & Flucytosine induced & 2.05 & 1.46 & 0.91 \\
\hline RPC25 & orf19.443 & DNA-direcred RNA polymerase III & 1.78 & 1.34 & 1.06 \\
\hline RPC40 & orf19.3564 & RNA polymerase & 1.73 & 1.00 & 0.89 \\
\hline \multicolumn{6}{|c|}{ Elongation factors } \\
\hline CAM1 & orf19.7382 & $\begin{array}{l}\text { Putative translation elongation factor eEF1 } \\
\text { gamma }\end{array}$ & 1.32 & 1.62 & 1.22 \\
\hline EFT2 & orf19.5788 & Elongation Factor 2 (eEF2) & 1.70 & 1.37 & 1.35 \\
\hline SUI2 & orf19.6213 & Putative translation initiation factor & 1.90 & 1.60 & 1.33 \\
\hline SUI3 & orf19.7161 & Putative translation initiation factor & 1.64 & 1.45 & 1.29 \\
\hline TFS1 & orf19.1974 & $\begin{array}{l}\text { Transcription is regulated upon yeast- } \\
\text { hyphal switch }\end{array}$ & 1.69 & 1.10 & 1.33 \\
\hline TEF1 & orf19.9009 & Translation elongation factor 1 -alpha & 1.74 & 1.67 & 1.12 \\
\hline TIF11 & orf19.5351 & Predicted translation initiation factor elF1a & 2.15 & 1.81 & 1.44 \\
\hline TIF1 & orf19.3324 & $\begin{array}{l}\text { Protein similar to } S \text {. cerevisiae translation } \\
\text { initiation factor }\end{array}$ & 1.67 & 1.00 & 0.99 \\
\hline TIF3 & orf19.3423 & Putative translation initiation factor & 1.63 & 1.24 & 1.09 \\
\hline \multicolumn{6}{|c|}{ Feric reductase and Feroxidase } \\
\hline CFL1 & orf19.1263 & Protein similar to ferric reductase Fre $10 p$ & 1.28 & 2.01 & 1.24 \\
\hline CFL2 & orf19.1264 & $\begin{array}{l}\text { Iron utilization protein; similar to an } S \text {. } \\
\text { cerevisiae oxidoreductase }\end{array}$ & 1.11 & 1.57 & 1.19 \\
\hline FET34 & orf19.4215 & Protein similar to multicopper ferroxidase & 1.05 & 1.71 & 1.16 \\
\hline \multicolumn{6}{|c|}{ Transcription factors } \\
\hline IPF29 & orf19.5975 & $\begin{array}{l}\text { Putative transcription factor with zinc finger } \\
\text { DNA-binding motif }\end{array}$ & 0.59 & 1.98 & 1.23 \\
\hline NUT2 & orf19.5268 & $\begin{array}{l}\text { Negative transcription regulator from artifical } \\
\text { reporters }\end{array}$ & 0.81 & 1.69 & 1.19 \\
\hline ZCF20 & orf19.11621 & $\begin{array}{l}\text { Predicted zinc-finger protein of unknown } \\
\text { function }\end{array}$ & 1.28 & 1.84 & 0.72 \\
\hline ZCF4 & orf19.1227 & $\begin{array}{l}\text { Putative transcription factor with zinc cluster } \\
\text { DNA-binding motif }\end{array}$ & 1.32 & 1.80 & 1.07 \\
\hline \multicolumn{6}{|c|}{$\begin{array}{l}\text { Down-regulated genes } \\
\text { Cell wall }\end{array}$} \\
\hline ALS2 & orf19.2122 & $\begin{array}{l}\text { ALS family protein includes cell-surface } \\
\text { glycoproteins }\end{array}$ & 0.52 & 0.67 & 0.88 \\
\hline BMH1 & orf19.3014 & $14-3-3$ protein, role in hyphal growth & 0.64 & 0.89 & 1.07 \\
\hline EAP1 & orf19.1401 & $\begin{array}{l}\text { Cell wall adhesin required for cell-cell } \\
\text { adhesion and biofilm formation }\end{array}$ & 0.32 & 0.99 & 1.18 \\
\hline ECE1 & orf19.3374 & Hyphal-specific expression & 0.65 & 0.86 & 0.84 \\
\hline \multicolumn{6}{|l|}{ ECE17 } \\
\hline HWP1 & orf19.1321 & Hyphal cell wall protein & 0.38 & 0.90 & 0.76 \\
\hline MNT1 & orf19.1665 & Alpha-1,2-mannosyl transferase & 0.58 & 0.80 & 0.96 \\
\hline PGA54 & orf19.2685 & Putative GPI-anchored protein & 0.32 & 0.69 & 1.07 \\
\hline PGA58 & orf19.4334 & $\begin{array}{l}\text { Putative GPI-anchored protein of unknown } \\
\text { function }\end{array}$ & 0.46 & 0.90 & 1.02 \\
\hline PGA62 & orf19.10281 & Putative GPI-anchored protein & 0.56 & 1.00 & 0.93 \\
\hline ALG7 & orf19.2187 & $\begin{array}{l}\text { Protein involved in cell wall mannan } \\
\text { biosynthesis }\end{array}$ & 0.77 & 0.65 & 0.98 \\
\hline ALS5 & orf19.5736 & Adhesin; ALS family protein & 0.85 & 0.55 & 0.92 \\
\hline $\mathrm{CHS} 4$ & orf19.7349 & Activator of Chs3p chitin synthase & 0.89 & 0.54 & 1.12 \\
\hline FGR41 & orf19.4910 & Putative GPI-anchored protein & 0.87 & 0.31 & 0.91 \\
\hline GLC3 & orf19.13067 & Similar to 1,4 -glucan branching enzyme & 0.71 & 0.58 & 1.07 \\
\hline RBT4 & orf19.13583 & Similar to plant pathogenesis-related & 0.83 & 0.54 & 0.69 \\
\hline
\end{tabular}




\begin{tabular}{|c|c|c|c|c|c|}
\hline & & proteins & & & \\
\hline ALS1 & orf19.5741 & $\begin{array}{l}\text { Adhesin; ALS family of cell-surface } \\
\text { glycoproteins }\end{array}$ & 0.63 & 0.63 & 0.89 \\
\hline ALS3 & orf19.2355 & ALS family protein & 0.37 & 0.48 & 0.70 \\
\hline ALS4 & orf19.4556 & ALS family protein & 0.52 & 0.55 & 1.00 \\
\hline FGR51 & orf19.156 & Protein lacking an ortholog in S. cerevisiae & 0.55 & 0.62 & 1.02 \\
\hline HYR1 & orf19.12440 & Nonessential, GPI anchored & 0.31 & 0.37 & 0.71 \\
\hline PGA63 & orf19.6217 & Putative protein of unknown function & 0.48 & 0.44 & 1.03 \\
\hline PMT1 & orf19.5171 & Protein mannosyltransferase & 0.52 & 0.52 & 0.92 \\
\hline PMT2 & orf19.6812 & Essential protein mannosyltransferase & 0.57 & 0.56 & 1.03 \\
\hline PMT4 & orf19.4109 & Protein mannosyltransferase & 0.35 & 0.47 & 0.91 \\
\hline RBT1 & orf19.1327 & $\begin{array}{l}\text { Putative cell wall protein with similarity to } \\
\text { Hwp1p }\end{array}$ & 0.29 & 0.42 & 0.72 \\
\hline CRH11 & orf19.2706 & Probable membrane protein & 0.87 & 0.52 & 0.58 \\
\hline IPF3964 & orf19.675 & Similar to cell wall proteins & 0.91 & 0.64 & 0.84 \\
\hline RBT4 & orf19.13583 & $\begin{array}{l}\text { Similar to plant pathogenesis-related } \\
\text { proteins }\end{array}$ & 0.83 & 0.54 & 0.68 \\
\hline TOS1 & orf19.1690 & $\begin{array}{l}\text { Protein described as similar to alpha } \\
\text { agglutinin anchor subunit }\end{array}$ & 0.67 & 0.65 & 0.91 \\
\hline WAL1 & orf19.6598 & $\begin{array}{l}\text { Protein required for hyphal growth and for } \\
\text { wild-type cell morphology, polarized } \\
\text { budding }\end{array}$ & 0.73 & 0.47 & 1.09 \\
\hline \multicolumn{6}{|c|}{ Glycolysis } \\
\hline ENO1 & orf19.395 & $\begin{array}{l}\text { Enolase (2-phospho-D-glycerate- } \\
\text { hydrolyase) }\end{array}$ & 0.54 & 1.02 & 0.97 \\
\hline FBA1 & orf19.4618 & Putative fructose-bisphosphate aldolase & 0.32 & 0.69 & 1.31 \\
\hline GLK1 & orf19.1408 & $\begin{array}{l}\text { Aldohexose specific glucokinase (by } \\
\text { homology) }\end{array}$ & 0.57 & 0.77 & 1.14 \\
\hline PGI1 & orf19.3888 & $\begin{array}{l}\text { Protein described as glucose-6-phosphate } \\
\text { isomerise }\end{array}$ & 0.61 & 0.73 & 0.91 \\
\hline ICL1 & orf19.6844 & Isocitrate lyase & 0.51 & 1.18 & 1.08 \\
\hline MLS1 & orf19.4833 & $\begin{array}{l}\text { Malate synthase; enzyme of the glyoxylate } \\
\text { cycle }\end{array}$ & 0.59 & 1.05 & 1.19 \\
\hline TDH3 & orf19.6814 & $\begin{array}{l}\text { Glyceraldehyde-3-phosphate } \\
\text { dehydrogenase }\end{array}$ & 0.49 & 1.04 & 1.34 \\
\hline TPI1 & orf19.6745 & Putative ortholog of $S$. cerevisiae Tpi $1 p$ & 0.47 & 0.72 & 0.91 \\
\hline ADH5 & orf19.2608 & Putative alcohol dehydrogenase & 0.96 & 0.62 & 0.71 \\
\hline GPM2 & orf19.1067 & Described as phosphoglycerate mutase & 0.71 & 0.44 & 0.93 \\
\hline $\mathrm{ADH} 2$ & orf19.5113 & Putative alcohol dehydrogenase & 0.47 & 0.54 & 0.95 \\
\hline GSY1 & orf19.3278 & Protein described as glycogen synthase & 0.37 & 0.26 & 0.69 \\
\hline PFK1 & orf19.3967 & Alpha subunit of phosphofructokinase (PFK) & 0.35 & 0.32 & 0.98 \\
\hline PFK2 & orf19.6540 & Beta subunit of phosphofructokinase (PFK) & 0.19 & 0.31 & 0.75 \\
\hline PGK1 & orf19.3651 & Phosphoglycerate kinase & 0.36 & 0.65 & 0.83 \\
\hline TPS2 & orf19.3038 & $\begin{array}{l}\text { Trehalose-6-phosphate (Tre6P) } \\
\text { phosphatise }\end{array}$ & 0.49 & 0.49 & 0.98 \\
\hline $\mathrm{ADH} 1$ & orf19.3997 & Alcohol dehydrogenase & 0.39 & 0.38 & 0.59 \\
\hline CDC19 & orf19.3575 & Putative pyruvate kinase & 0.20 & 0.34 & 0.49 \\
\hline PDC11 & orf19.2877 & Protein similar to pyruvate decarboxylase & 0.29 & 0.37 & 0.63 \\
\hline \multicolumn{6}{|c|}{ Secretory pathway } \\
\hline $\mathrm{CHC} 1$ & orf19.3496 & Clathrin heavy chain & 0.47 & 0.90 & 1.20 \\
\hline ERV29 & orf19.4579 & $\begin{array}{l}\text { Similar to Saccharomyces cerevisiae } \\
\text { Erv29p ER-Golgi transport vesicle protein }\end{array}$ & 0.60 & 0.69 & 1.06 \\
\hline SEC26 & orf19.528 & $\begin{array}{l}\text { Beta chain of secretory vesicles coatomer } \\
\text { complex }\end{array}$ & 0.57 & 0.67 & 1.18 \\
\hline SEC9 & orf19.7764 & Transport protein & 0.54 & 0.59 & 1.06 \\
\hline SEC11 & orf19.3259 & Signal peptidase subunit & 0.56 & 0.52 & 0.94 \\
\hline SEC16 & orf19.11823 & Multidomain vesicle coat protein & 0.59 & 0.64 & 1.01 \\
\hline SEC23 & orf19.1254 & $\begin{array}{l}\text { Transcription is regulated upon yeast- } \\
\text { hyphal switch }\end{array}$ & 0.63 & 0.71 & 1.05 \\
\hline SEC24 & orf19.12194 & Possible role in ER to Golgi transport & 0.51 & 0.65 & 1.06 \\
\hline SEC61 & orf19.6176 & Essential protein & 0.51 & 0.46 & 0.93 \\
\hline \multicolumn{6}{|c|}{ Transport } \\
\hline CAN2 & orf19.111 & Putative amino acid permease & 0.55 & 0.67 & 0.84 \\
\hline CRP1 & orf19.4784 & $\begin{array}{l}\text { Copper transporter of the plasma } \\
\text { membrane }\end{array}$ & 1.21 & 0.61 & 0.77 \\
\hline GAP1 & orf19.4304 & General amino acid permease & 1.02 & 0.52 & 1.18 \\
\hline GAP6 & orf19.6659 & General amino acid permease & 1.11 & 0.65 & 0.91 \\
\hline HAK1 & orf19.6249 & Putative potassium transporter & 0.99 & 0.60 & 1.11 \\
\hline IFC1 & orf19.3746 & Oligopeptide transporter & 0.78 & 0.56 & 0.88 \\
\hline IFC4 & orf19.2292 & Oligopeptide transporter & 0.80 & 0.66 & 0.86 \\
\hline GNP1 & orf19.8784 & Asparagine and glutamine permease & 0.60 & 0.35 & 0.92 \\
\hline HGT6 & orf19.2020 & Putative glucose transporter & 0.56 & 0.59 & 0.70 \\
\hline HGT7 & orf19.2023 & Putative glucose transporter & 0.61 & 0.61 & 0.75 \\
\hline HGT8 & orf19.2021 & Putative glucose transporter & 0.56 & 0.55 & 0.69 \\
\hline
\end{tabular}




\begin{tabular}{|c|c|c|c|c|c|}
\hline \multicolumn{6}{|c|}{ Multi Drug and other transporter } \\
\hline CDR1 & orf19.6000 & $\begin{array}{l}\text { Multidrug transporter of ATP-binding } \\
\text { cassette }\end{array}$ & 1.24 & 0.52 & 1.02 \\
\hline CDR2 & orf19.5958 & Multidrug transporter, ATP-binding cassette & 1.14 & 0.51 & 1.08 \\
\hline CDR4 & orf19.5079 & Putative transporter of ATP-binding cassette & 0.81 & 0.66 & 0.89 \\
\hline QDR1 & orf19.8138 & Putative transporter of antibiotic resistance & 0.52 & 0.72 & 0.87 \\
\hline CRP1 & orf19.4784 & $\begin{array}{l}\text { Copper transporter of the plasma } \\
\text { membrane }\end{array}$ & 1.21 & 0.61 & 0.77 \\
\hline IFC1 & orf19.3746 & Oligopeptide transporter & 0.78 & 0.56 & 0.88 \\
\hline IFC4 & orf19.2292 & Oligopeptide transporter & 0.95 & 0.66 & 0.97 \\
\hline HAK1 & orf19.6249 & Putative potassium transporter & 0.73 & 0.60 & 0.86 \\
\hline \multicolumn{6}{|c|}{ Transcription factors } \\
\hline GAL4 & orf19.5338 & Putative transcription factor with zinc cluster & 0.52 & 0.77 & 1.10 \\
\hline IPF29 & orf19.5975 & Putative transcription factor with zinc finger & 0.44 & 1.59 & 1.23 \\
\hline SKO1 & orf19.1032 & Putative transcription factor & 0.61 & 0.77 & 1.08 \\
\hline $\mathrm{CPH} 2$ & orf19.1187 & Transcriptional activator of hyphal growth & 0.72 & 0.58 & 0.90 \\
\hline GAT2 & orf19.405 & Putative DNA-binding transcription factor & 1.19 & 0.44 & 0.96 \\
\hline IPF16124 & orf19.173 & Putative transcription factor with zinc finger & 0.73 & 0.63 & 0.87 \\
\hline MOT1 & orf19.4502 & Transcriptional accessory protein & 0.75 & 0.64 & 0.99 \\
\hline MSN4 & orf19.4752 & Putative zinc finger transcription factor & 0.68 & 0.63 & 0.92 \\
\hline ZCF1 & orf19.255 & $\begin{array}{l}\text { Predicted zinc-cluster protein of unknown } \\
\text { function }\end{array}$ & 0.82 & 0.53 & 1.10 \\
\hline ECM22 & orf19.2623 & $\begin{array}{l}\text { Putative protein involved in cell wall } \\
\text { biogenesis }\end{array}$ & 0.61 & 0.63 & 0.91 \\
\hline HAC1 & orf19.2432 & Putative transcription factor & 0.43 & 0.54 & 0.91 \\
\hline SSN6 & orf19.6798 & $\begin{array}{l}\text { Functional homolog of S. cerevisiae } \\
\text { Cyc8p/Ssn6p }\end{array}$ & 0.56 & 0.60 & 1.01 \\
\hline TEC1 & orf19.5908 & TEA/ATTS transcription factor & 0.64 & 0.44 & 1.01 \\
\hline UME6 & orf19.9381 & $\begin{array}{l}\text { Transcription factor; required for wild-type } \\
\text { hyphal extension }\end{array}$ & 0.36 & 0.36 & 0.74 \\
\hline \multicolumn{6}{|c|}{ Cell polarity and signal transduction } \\
\hline ARP3 & orf19.2289 & $\begin{array}{l}\text { Shows Myo5p-dependent localization to } \\
\text { cortical actin patches at hyphal tip }\end{array}$ & 0.28 & 0.52 & 1.01 \\
\hline ARP8 & orf19.10867 & $\begin{array}{l}\text { Mutation confers hypersensitivity to toxic } \\
\text { ergosterol analog, and to amphotericin B }\end{array}$ & 0.64 & 0.35 & 1.06 \\
\hline BEM1 & orf19.4645 & $\begin{array}{l}\text { Protein required for wild-type budding, } \\
\text { hyphal growth, and virulence in a mouse } \\
\text { systemic infection }\end{array}$ & 0.52 & 0.62 & 1.03 \\
\hline BEM2 & orf19.6573 & $\begin{array}{l}\text { Putative Rho1p GTPase activating protein } \\
\text { (GAP); serum-induced transcription }\end{array}$ & 0.36 & 0.36 & 0.86 \\
\hline BUD2 & orf19.940 & GTPase activating protein (GAP) for Rsr1p & 0.50 & 0.44 & 0.91 \\
\hline CLC1 & orf19.4594 & Clathrin light chain (by homology) & 0.63 & 0.58 & 0.88 \\
\hline MYO2 & orf19.5015 & Class V myosin & 0.44 & 0.58 & 1.13 \\
\hline RAS1 & orf19.1760 & RAS signal transduction GTPase & 0.57 & 0.59 & 0.89 \\
\hline RGS2 & orf19.695 & Protein of RGS superfamily & 0.41 & 0.53 & 1.03 \\
\hline TPM2 & orf19.6414.3 & Protein described as tropomyosin isoform 2 & 0.61 & 0.60 & 0.89 \\
\hline
\end{tabular}

The expression of 265 genes was affected by a factor of $\geq 1.5$ in the hat $1 \Delta$, hat $2 \Delta$ double mutant after $60 \mathrm{~min}$; among these, 97 and 168 genes were up- and downregulated respectively.

The main categories of up-regulated genes were genes belong to amino acid biosynthesis (ARG1, ARG3, GAP4, MET14, MET3, SAM2), ferric reductases and ferroxidases (CFL1, CFL2, FET34), cell wall ALG8, CSE1, IFF5, PGA26, PGA59) and transcription factors (IPF29, NUT2, ZCF20, ZCF4). Genes involved in multidrug and other transporter (CDR1, CDR2, CDR4, CRP1, QDR1, IFC1, IFC4, HAK1), genes encoding for cell wall proteins (ALG7, ALS5, CHS4, FGR41, GLC3, IPF3964, RBT4, TOS1, WAL1), transcription factors (CPH2, GAT2, IPF16124, MOT1, MSN4, ZCF1) were down-regulated after 60 min of incubation.

The expression of 29 genes was affected by a factor of $\geq 1.5$ after $180 \mathrm{~min}$, with 10 and 19 genes being up- and down-regulated respectively. Of the ten ORFs, two genes 
involved in cell wall, RBT5, YWP1, two genes encoding for putative acetyl-CoA synthetase $A C H 1, A C S 1$ and the rest were AOX2 (Alternative oxidase), FAS1 (beta subunit of fatty-acid synthase), IPF8464, RPL20B (predicted ribosomal protein), RPN12 (26S proteasome regulatory subunit) and TUB2 (Beta-tubulin). The downregulated genes were two genes encoding for cell wall and cell surface proteins (AAF1 and GSL1), three genes involved in transport (IPF15119, IPF9079 and SHA3) and the rest involved in other functions (Supplementary table 2.8).

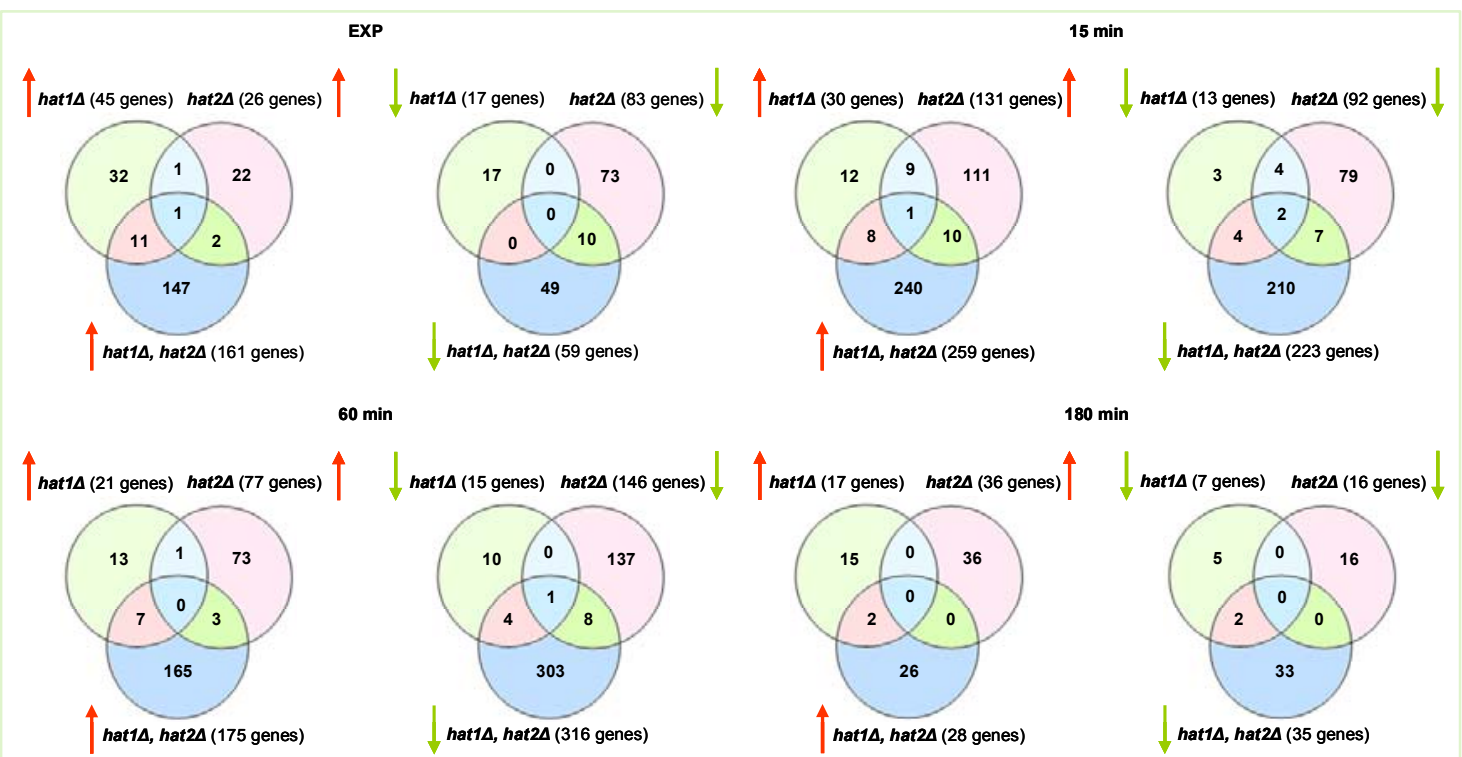

Figure 2.18 Venn diagrams of genes regulated by Hat $1 p$ and Hat2p in the simple and double mutants. The CAI4 strain was used as reference for the hat $2 \Delta$ simple and hat $1 \Delta$, hat2 $\Delta$ double mutant (MAH4), which were all grown in identical conditions: $\mathrm{YNB} / 28^{\circ} \mathrm{C}(\mathrm{EXP})$ and Lee $/ 37^{\circ} \mathrm{C}$ (Inducing).

\section{Discussion}

In S. cerevisiae HAT-B complex is composed of three proteins, Hat1, Hat2 and Hif1 ( \&. Parthun, 2004; Poveda et al., 2004). Hat2p acts as a bridge between Hat1 and Hif1 proteins (Poveda et al., 2004) and is required for high affinity binding of Hat1p to histone H4 (Parthun et al., 1996). It seems to be essential for all the functions carried out by Hat $1 p$ because deletion of HAT2 always produces similar defects to those of HAT1 (Kelly et al., 2000 b; Parthun et al., 1996; Ruiz-Garcia et al., 1998).

In our laboratory the HAT1 and HAT2 were deleted in a CAI4 background strain. Morphologically hat1 $\triangle$ mutant is very different to the CAl4 strain (Degano, Ph.D thesis in process) and the culture of the hat $1 \triangle$ mutant in YEPD liquid medium is composed of a mixture of large cells and large chains of elongated, aberrantly shaped cells attached to one another. In contrast, the hat $2 \Delta$ mutant doesn't show any significant difference in growth rate, cell size and morphology. 
The focus of our work understood the possible role of HAT1 and HAT2 on the expression of genes that are associated with morphogenesis in the $C$. albicans. If Hat2p has additional roles to those described for Hat1p, it seems likely that this fact would be reflected in the transcriptional profile. With the aim of give some light on this, we analysed the transcriptional profile of the hat $2 \Delta$ and hat $1 \Delta$, hat $2 \Delta$ mutants. The transcriptional profile of the hat1 $\Delta$ mutant during the yeast mode of growth and during the yeast-hypha transition has been carried out in our laboratory. We have deleted the HAT1 gene in a hat2 $\triangle$ mutant background strain. As a result, we were able to perform a comprehensive phenotypic and transcriptional analysis of hat $1 \Delta$ and hat $2 \Delta$ simples and double mutants, and in particular we were able to assess the relative contributions of both proteins to growth and morphogenesis in C. albicans. Recently, it has been reported that three tagged protein components of the HAT-B enzyme are mainly located in the nucleus (Poveda et al., 2004). However, in several species Hat1p has also been detected in the cytoplasm (Lusser et al., 1999; Ruiz-Garcia et al., 1998; Verreault et al., 1998). Our results are different to those described for S. cerevisiae. In this organism deletion of any of HAT1 or HAT2 gene produce a similar phenotype (Kelly et al., 2000b; Parthun et al., 1996; Ruiz-Garcia et al., 1998) while we obtained different phenotypes and transcriptomic profiles.

For obtaining the location of Hat1p and Hat2p in $C$. albicans, we carried out a fusion construct to the green fluorescent protein (GFP). This has allowed us to compare our data with the Hat-B proteins of $S$. cerevisiae, which represent the only known complete set of Hat proteins in a fungal species. Our results clearly show that, in C. albicans a dominant portion of the putative HAT-B complex is also located in the nucleus.

Next, to further characterize the sub-cellular localization of the two HAT-B components, we asked whether their nuclear residence is interdependent. To address this question and the sub-cellular localization of each fused component, Hat1-GFP and Hat2-GFP, were visualized in yeast cells defective in any of the other opposite proteins. The nuclear residence of Hat1-GFP exhibited no difference between cells with a wild-type. But the nuclear localization of the Hat2-GFP depends on HAT1. In the hat1 $\Delta$ strain, the Hat2-GFP signal was visualized throughout the cell. Therefore, Hat1p may participate in the nuclear localization of the catalytic subunit Hat2p. Opposite to our results, it has been reported that in $S$. cerevisiae the nuclear localization of the Hat $1 p$ depends on HAT2 and Hat2p participate in the nuclear localization of the catalytic subunit Hat1p (Poveda et al., 2004).

Our result describes a difference in the interdependency of Hat $1 p$ and Hat $2 p$ between S. cerevisiae and C. albicans. In the C. albicans like S. cerevisiae (Kleff et al., 1995; 
Parthun et al., 1996; Poveda et al., 2004) single hat2s mutant lack of obvious phenotype or growth defects. Contrary to $S$. cerevisiae, the $C$. albicans hat1 $1 \Delta$ mutant presents a phenotype with growth defects. Morphologically, the double mutant like the hat $1 \Delta$ simple mutant is very different to CAl4 as is shown in Fig. 2.5. The hat $1 \Delta$, hat2 $\Delta$ double mutant like hat1 $\Delta$ simple mutant failed to form true hypha in all the liquid media assayed (Fig. 2.7). We found that the hat1 $\Delta$ and double mutant were more sensitive than the CAl4 and hat $2 \Delta$ to $1 \mathrm{M}$ of $\mathrm{NaCl}, 1 \mathrm{mg} / \mathrm{ml}$ of CFW, $0.5 \mathrm{mg} / \mathrm{ml}$ of Congo red and $15 \mathrm{mM}$ of Caffeine (Fig. 2.9). These results suggest that Hat1p play an important role in C. albicans. We have identified by two-hybrid experiment that Hat1p and Hat2p doesn't interact directly. We haven't detected any homology of ScHIf1 in the C. albicans Genome Data Base or in any other organism. The results of sub-cellular location and of two-hybrid analysis suggest that there are other protein o proteins that mediate one interaction between Hat1p and Hat $2 p$ in $C$. albicans. The determination of the putative bridge protein is under investigation. We analysed the transcriptional profile of hat $2 \Delta$ and hat $1 \Delta$, hat $2 \Delta$ double mutant during the yeast mode of growth and the yeast-hypha transition at 15, 60 and $180 \mathrm{~min}$. We used CAI4 (Fonzi \& Irwin, 1993) as wild-type strain, because all of mutant strains were derived from this strain. Morphologically, deletion of HAT1 has been known to be exerting relatively strong phenotypic effects upon C. albicans (Degano, Ph.D thesis in process). Then, it is surprising that deletion of HAT1 led to few changes in the transcriptional profile relative to that of the hat $2 \Delta$ mutant. However, this was in contrast with the minimal phenotypic effects of the hat2 $\Delta$ deletion.

109 genes were found to be regulated in the hat $2 \Delta$ mutant during the yeast mode of growth. The major portion of genes up-regulated during the yeast mode of growth in the hat $2 \Delta$ mutant included genes involved in respiration and histone assembly. Seven genes involved in respiration were found to be up-regulated in the hat $2 \Delta$ mutant. We observed eleven down-regulated genes belonging to the glycolysis and TCA cycle functional category. It has been suggested that genes involved in central metabolism and energy generation are stimulated in cells with defects in the cell wall (Lagorce et al., 2003). In the hat $2 \Delta$ strain it is noteworthy that five genes encoding for cell wall proteins show a significant alteration in their expression level. Although the number of down-regulated genes is greater than the number of up-regulated ones, the upregulated genes show changes of $1.50-$ to 3.30 -fold. In the case of the down-regulated genes, only, RPS8A (Niewerth et al., 2003), RPPO (Lorenz et al., 2004), PGA62 (De Groot et al., 2003), IPF6342 (Copping et al., 2005) and PGA59 show a higher change than 3.70-fold. YWP1 (Sohn et al., 2003) being the most affected with a 5.88-fold 
transcriptional reduction, while the remaining genes only just surpass the threshold of a factor of $>1.5$. These groups of genes are not reported to be regulated in the hat1 $\Delta$ mutant. It is difficult to predict the overall effect of the deletion of hat $2 \Delta$ on the cell respiration and energy generation, but the $16 \%$ of the altered genes belong to this functional group suggesting quite a close relationship between the expression of HAT2 and some of the central metabolic pathways.

In the hat $1 \Delta$, hat $2 \Delta$ double mutant, ten of the genes encoding for cell wall proteins that show an altered expression level were up-regulated including four genes that encode putative GPI-anchored protein (PGA10, PGA14, RBT5, RHD3) (de Groot et al., 2004; Kadosh \& Johnson, 2001). Since only three genes (ECM17, IFF5, PGA49), of this functional group are down-regulated, the data suggest that deletion of the HAT1 and HAT2 genes regulate possible cell wall genes involved in the normal cell wall formation.

Also the fact that the TLO1, TLO3, TLO11, TLO12 and TLO16 genes, which are members of a family of telomere-proximal genes showed a significantly induced expression level, and that eight genes belonging to this family (TLO2, TLO3, TLO4, TLO9, TLO10, TLO11, TLO12 and TLO34) were up-regulated in hat1A mutant (Degano, Ph.D thesis in process), could be related to a putative role of HAT1 in telomeric silencing in C. albicans. In S. cerevisiae deletion of HAT1 alone doesn't directly affect on telomeric silencing but in combination with specific histone $\mathrm{H} 3$ amino terminal tail mutations, results in a significant defect in telomeric silencing (Kelly et a/., $2000 \mathrm{~b}$ ). This result suggested that the function of C. albicans Hat $1 \mathrm{p}$ to regulation of telomeric silencing is distinct of those reported for $S$. cerevisiae Hat-B complex (Poveda et al., 2004).

The transcriptional profile of the hat $2 \Delta$ mutant was very different from the hat $1 \Delta$ mutant during the yeast-hypha transition. Expression of 429 genes (196 up- and 233 downregulated) was affected after 15, 60 and 180 min of incubation. By contrast a lower number of genes (54 up- and 34 down-) have been reported to be regulated by the deletion of HAT1 during the same times of incubation and only eighteen genes were common. These results suggest that Hat $2 p$ has different roles to those of Hat $1 p$, and this fact is reflected in the transcriptional profile during the yeast-hypha transition present in this work. Deletion of HAT2 results in only two common genes up-regulated at the three times of incubation. This result indicates a strong sequential alteration of the transcriptional profile in this mutant, a fact that differs from the behavior of other mutants analyzed by our research group. We have detected alteration of nineteen genes encoding for transcription factors, seven up- and twelve down-regulated at any 
of the times assayed. Among the up-regulated genes three -CTA2, CTA24, CTA29 (Doedt et al., 2004; Kaiser et al., 1999; van het Hoog et al., 2007; Zakikhany et al., 2007 )- encoding for putative transcriptional activators were up-regulated after $180 \mathrm{~min}$ and have been reported to be repressed by Efg1p. One gene, IPF19804 (Karababa et al., 2004) is an ortholog of S. cerevisiae TFC3, a putative DNA-binding transcription factor; with a decreased transcription upon fluphenazine treatment (Nobile \& Mitchell, 2005) were up-regulated at $15 \mathrm{~min}$. The other two genes were MRR1, an ortholog of $S$. cerevisiae HAP1, which is a Zinc finger transcription factor (Morschhauser et al., 2007) (at 60 min), GAL4 (Keng, 1992) which is a putative transcription factor with zinc cluster DNA-binding motif; ortholog of the S. cerevisiae GAL4 gene (Martchenko et al., 2007) (at $180 \mathrm{~min}$ ). GAL4 appeared as up-regulated during the three times of incubation in the gcn5 $\Delta$, hda1 $\Delta$ double mutant (Rashki, Ph.D thesis 2009). Of the twelve downregulated transcription factors, only $H A C 1$ has been reported to be down-regulated in the hat1 $\Delta$ mutant at 15 min of incubation (Degano, Ph.D thesis in process). We have found this gene also down-regulated in the hat $1 \Delta$, hat $2 \Delta$ double mutant confirming both results. However the HAC1 gene was up-regulated after 15 and $60 \mathrm{~min}$ in the transcriptional response of CAl4 to temperature (Rashki, Ph.D thesis 2009). As those are opposite results, the HAC1 gene is a strong candidate to be used as master gene for analyzing the role of the acetylation process in the yeast-hypha transition.

We have found changes in the transcript levels of eighteen genes encoding for cell wall proteins including nine genes BMT5 (at $15 \mathrm{~min}$ ), IRS4, FGR41 (at $60 \mathrm{~min}$ ), HWP1, PGA54 (at $180 \mathrm{~min}$ ), BMT9, CHS2, GSL1 (at 15 and $60 \mathrm{~min}$ ), PGA45 (at 15 and 180 min) up-regulated; and nine genes PHR1, TOS1 (at $15 \mathrm{~min}$ ), ALS4, ECM31, HYR3, PGA6, PGA63 (at $60 \mathrm{~min}$ ), HYR1 (at $180 \mathrm{~min}$ ), GSC1 (at 15 and $60 \mathrm{~min}$ ) downregulated in the hat2 $\triangle$ mutant. However eight genes, ALS2, ALS4, FRE10 (at $60 \mathrm{~min}$ ), PIR1 (at $180 \mathrm{~min}$ ), PGA13 (at 15 and $60 \mathrm{~min}$ ), PGA62, YWP1 (at three times), SIM1 (at 60 and $180 \mathrm{~min}$ ), and seven genes, PMT1, SUN41, TOS1 (at $15 \mathrm{~min}$ ), ECM33, HWP1, $I D H 1$ (at $60 \mathrm{~min}$ ), ALS3 (at 15 and $60 \mathrm{~min}$ ) were found to be regulated in the same conditions in the hat1 $\Delta$ mutant. As we have not found coincident genes, our results prove again that the hat $2 \Delta$ mutant has a distinct transcriptome of the hat $1 \Delta$ mutant.

The regulation of seventeen genes (13 up-regulated $A D H 1, A D H 5, F B A 1, G P H 1$, GPM1, GLG1, HXK2, PCK1, PDC11, PFK2, PGK1, TPI1 and ENO1 after 15 min) involved in the glycolytic pathway was detected. Expression ten of them (ADH1, $A D H 5$, FBA1, GPH1, GPM1, PDC11, PFK2, PGK1, TPI1 and ENO1) have been downregulated after $15 \mathrm{~min}$ in $C$. albicans transcription response to temperature. The genes involved in glycolytic pathway (FBA1, GPM1, PFK2, PGI1, TPI1 and HXK2) have been 
reported to be down-regulated in the APSES mutants, gcn5 5 , hda $1 \Delta$ and odc $\Delta$ mutants during the hypha transition (Doedt et al., 2004; Rashki, Ph.D thesis 2009) suggesting a specific regulation that deserves further investigation.

Fifteen genes encode for ribosomal proteins were found to be regulated in the hat2 $\Delta$ mutant during the yeast-hypha transition. No genes of the ribosomal category have been found to be up- or down-regulated in the hat1 $\Delta$ mutant (Degano, Ph.D thesis in process).

The transcriptional profile of hat $1 \Delta$, hat $2 \Delta$ double mutant was very different from those found on both the hat $1 \Delta$ and hat $2 \Delta$ simple mutants during the yeast-hypha transition.

Expression of 793 genes was affected after 15, 60 and 180 min of incubation. Forty seven genes involved in ribosomal proteins: cytoplasmic (31 genes) and mitochondrial (7 genes) or putative translation initiation factor (9 genes) were found to be upregulated in the hat $1 \Delta$, hat $2 \Delta$ double mutant (31 at $15 \mathrm{~min}, 4$ at $60 \mathrm{~min}, 9$ at 15 and 60 $\min , 1$ at 60 and $180 \mathrm{~min}$ and 2 at three times of incubation) and the expression of almost all of them decreased at $180 \mathrm{~min}$.

None of those genes have been reported to be regulated in the hat $1 \Delta$ mutant, while we have found five genes (RPL11, RPL20B, RPS4A, RPL10 and RPL14B) were downregulated in the hat $2 \Delta$ mutant.

It is difficult to predict the overall effect of the deletion of both genes, HAT1 and HAT2, on the regulation of genes encoding for ribosomal proteins, but the fact that almost one third of the genes (47 out of 143) encoding for ribosomal proteins and that a 5.9\% (47 genes out of the total regulated genes, 793) suggests that Hat1p and Hat2p, jointly regulate expression this set of genes and this point also deserves further investigation.

Regarding the functions of other genes, we observed that expression of 131 common down-regulated genes after 15 and $60 \mathrm{~min}$ of incubation. Of those the main representative categories were genes encoding for cell wall proteins (12) -ALS1 (Hoyer et al., 1995), ALS3 (Hoyer et al., 1998a), ALS4 (De Groot et al., 2003), ECM21 (Karababa et al., 2004; Nobile et al., 2003), FGR51 (Uhl et al., 2003), HYR1 (Bailey et al., 1996), PGA4 (Eisenhaber et al., 2004), PGA63 (De Groot et al., 2003), PMT1, PMT2, PMT4 (Cantero et al., 2007; Timpel et al., 1998) and RBT1 (Sohn et al., 2003)- , seven genes encoding for enzymes glycolytic pathway (ADH2, PFK1, PFK2, GPM1, GSY1, PGK1and TPS2), three genes involved in glucose transport (HGT6, HGT7 and $H G T 8)$, ten genes involved in the cell polarity and signal transduction (ARP3, ARP8, BEM1, BEM2, BUD2, CLC1, MYO2, RAS1, RGS2 and TPM2), six genes involved in the secretory pathway (ERV46, SEC11, SEC16, SEC24, SEC61, SEC9) and five transcription factors including ECM22, HAC1, SSN6, TEC1 and UME6. 
Down-regulation of nine genes (ARP3, BUD2, CLC1, MYO2, RAS1, TPM2, ERV46, SEC16 and SEC24) involved in the cell polarity and signal transduction and secretory pathway have also been reported in the odc $\Delta$ mutant in C. albicans (Rashki, Ph.D thesis 2009). Those results suggest a coordinate regulation of this set of genes by acetyltranferases and polyamines.

Up-regulation the HAC1, TEC1 and UME6 genes have been reported for C. albicans as transcriptional response of to temperature (Rashki, Ph.D thesis 2009).

Regulated genes with known functions in the hat1 $\Delta$, hat $2 \Delta$ double mutant do not share significant common functional categories (see tables 2.4 and 2.7) with those of hat1 $\Delta$ and hat $2 \Delta$ simple mutants suggesting that Hat $1 p$ and Hat $2 p$ jointly regulates expression a large number of genes in $C$. albicans.

In summary, despite the fact that both are putative subunits of the HAT-B complex, hat $1 \Delta$ and hat $2 \Delta$ mutants displayed different transcriptional profile in the both the yeast mode of growth and during the yeast-hypha transition suggesting that Hat $1 p$ and Hat2 $p$ regulate separately different set of genes in $C$. albicans. The study of transcriptional profile of the hat $1 \Delta$, hat $2 \Delta$ double mutant revealed that a few genes were overlapped with both hat $1 \Delta$ ( 27 genes) and hat $2 \Delta$ ( 28 genes) simple mutants. Our results suggest that, in addition of regulation different set of genes, Hat1 $p$ and Hat2p function jointly in $C$. albicans regulating another specific set of genes. As in our case, in $S$. cerevisiae it has been reported that hat $1 \Delta$ and hat $2 \Delta$ mutants display different transcriptional profiles during exponential growth in a glucose-rich medium (Rosaleny et al., 2005) and the analysis of the double mutant showed as in our case a higher number of regulated genes than those of the single mutants. In $S$. cerevisiae, the study of the GO categories in the double mutant revealed that their transcriptome is more similar to that observed in the hat1 $\Delta$ mutant however the number of genes and the fold changes observed are higher in the double mutant (Rosaleny et al., 2005). In our case we can not support such a hypothesis because although regarding the phenotypic analysis the double mutant is more similar to the hat $1 \Delta$ mutant this is not the case for the transcriptional profile.

To summarize, in $S$. cerevisiae the proteins Hat $1 p$ and Hat $2 p$ undoubtedly participate in common processes, such as Lys12 acetylation of free $\mathrm{H} 4$ histone (Poveda et al., 2004; Rosaleny et al., 2005). The role of C. albicans Hat1p and Hat2p in acetylation of histones is under investigation. However, our data suggested that both proteins may also have functions regulating genes involves in cell physiology as could be deduced by the phenotypes. Up to now it is not clear what those roles could be. Nevertheless, this is not the first case that HAT complexes composed of subunits participate in 
different functions. In fact, this appeared to be a general feature of the HATs proteins forming HAT complexes like NuA3, INO80, etc. Our results for the $C$. albicans, HAT-B putative complex suggest, as have been described for other HATs complexes composed by subunits, that it is implicated in more than one function. 
Phenotypic, virulence and transcriptome analysis of the Candida albicans gcn $5 \Delta$, $\sin 3 \Delta$ double mutant

\section{Abstract}

C. albicans Gcn5p and $\operatorname{Sin} 3 p$ proteins regulate morphogenetic process, including filamentation and chlamidospore formation. The SIN3 gene of Candida albicans was disrupted in a gcn5 $\Delta$ background to investigate the role of a $C$. albicans double mutant $g c n 5 \Delta, \sin 3 \Delta$ in growth, morphogenesis and pathogenicity. The behavior of the double $g c n 5 \Delta, \sin 3 \Delta$ mutant resembled that of both simple mutants with respect to hyphal outgrowth and chlamydospore formation. For morphogenesis on solid media and sensitivity to Caffeine, Hygromycin, Amphotrecin B, Itraconazole and Fluconazole, the gcn5 5 phenotype appeared as the dominant phenotype in the double mutant. Wholegenome microarray analysis of the $g \operatorname{cn} 5 \Delta, \sin 3 \Delta$ double mutant revealed an altered expression of 558 and 1271 genes during the yeast mode of growth and during the yeast-hypha transition respectively. The main categories of up-regulated genes growing as yeast were oxidative stress response, DNA synthesis and replication, chitin anabolism. The main down-regulated genes correspond to those coding for cell wall proteins, electron transport, ribosomal proteins and transcriptional factors.

Forty-three genes were common to the three times of the yeast-hypha transition assayed (15, 60 and $180 \mathrm{~min}$ ); 156 genes were common between 15 and $60 \mathrm{~min} ; 47$ at 60 and $180 \mathrm{~min}$. Some of genes involved in cell wall (8 genes), fatty acid metabolism (5 genes), oxidase/peroxidase (4 genes) and transcription factors (2 genes) were downregulated at three times of incubation. The main common genes at 15 and $60 \mathrm{~min}$ encode for cell wall proteins (ALS4, GCS1, HYR1, MNT1, MNT2, PGA54, PMT1, PMT4, RBT4 and SUN41), transcriptional factors (ARG83 and ECM22) were downregulated and genes in the category of mitochondrial structure (AMI3, MCR1, NAD1, PST2, SOD2 and YOR100) and transcriptional factors (GCN4, PDC2 and RPN4) were up-regulated. Finally at 60 and $180 \mathrm{~min}$ of incubation, we have found down-regulation of genes involved in electron transport and respiration (ATP1, ATP14, COX5A, NDE1 and STF2), transporters (CDR1, IPF12884, MTR and ZRT2) and a transcription factor (TYE7). At one time of incubation, differentially expressed genes represented functions as diverse as mitochondrial, vitamin biosynthesis, ergoestrol biosynthesis, glucose transporters, histone, cell wall, cell polarity, signal transduction and ribosomal proteins. The results presented here include a global transcriptome analysis of the cellular response to the double deletion of the GCN5 and SIN3 genes and afford a basis for a 
better understanding of the putative interaction between histone acetyltransferases and co-repressors.

\section{Introduction}

In eukaryotes, the transition between alternative developmental pathways is mainly attributed to a switch in transcriptional programs. It has been postulated different modes of regulation at a transcriptional level mediated by processes of histone deacetylation and acetylation (Pnueli et al., 2004). There are numerous histone acetyltransferases (HATs) and histone deacetylases (HDACs), even in a simple organism such as yeast. At present, the functions of most of these enzymes and their putative role as transcriptional regulators are not clearly understood. Even less is known about their functions in processes other than transcription. The challenge now is to elucidate, in molecular detail the mechanisms by which and the extent to which, the different HATs and HDACs participate in all the biological processes. In the case of yeast, its small size and the availability of its genome sequence and cDNA microarray have facilitated the recent genome-wide approaches for the comprehensive determination of the functions of HATs and HDACs.

Eukaryotic GCN5 acetyltransferases influence diverse biological processes by acetylating histones and non-histone proteins and regulating chromatin and genespecific transcription as part of multi-protein complexes. In lower eukaryotes and invertebrates these complexes include the ADA complex that is still incompletely understood (Wang et al., 2008 ). In yeast, Gcn5p exists as part of complexes of two basic types: the small ADA and the larger SAGA (Spt-Ada-Gcn5 acetyltransferase) complexes (Lee \& Workman, 2007). While in the ADA complex data are scarce, it is known that the yeast SAGA complexes function mostly as coactivators that acetylate nucleosomal histones $\mathrm{H} 3$ and $\mathrm{H} 2 \mathrm{~B}$ and facilitate chromatin remodelling, transcription, nuclear export of mRNAs, and nucleotide excision repair (Baker \& Grant, 2007). C. albicans GCN5 encodes a protein with 449 amino acid and little is known about the regulation of its relative expression in biological process including its role in development. In our laboratory the GCN5 gene was deleted in a CAI4 background strain. Deletion of GCN5 appeared to block hyphal formation in a variety of standard inducing conditions, for example in the presence of serum or GlcNAc or during growth on Spider medium (Degano, Ph.D thesis in process). The function of GCN5 on the alternative pathway of filamentation in C. albicans is yet unknown.

SIN3 is a component of histone deacetylase complex known to be important for transcriptional repression and that is conserved from yeast to human (Silverstein \& 
Ekwall, 2005). While multiple isoforms of SIN3 have been reported, little is known about their relative expression. SIN3 is believed to serve as a scaffold protein for assembly of the complex and has been shown to be the major subunit that targets the complex to specific promoters (Silverstein \& Ekwall, 2005). Null mutation in C. albicans $\sin 3 \Delta$ results in a slight growth defect and also fails to undergo hypha formation (switching-defective) under all assayed conditions (Monterola, Ph.D thesis 2002).

In the present work we show that deletion of the GCN5 and SIN3 as double mutant like both simple mutants is associated with a difference between the growth rate of the double mutant and CAI4 in liquid medium at $28^{\circ} \mathrm{C}$. Morphologically, the double mutant, like both simple mutants, is very different to the CAI4 strain. The main phenotype of the double mutant is the inability to switch from the yeast to the filamentous form in liquid media containing serum or at $37^{\circ} \mathrm{C}$ and in all the solid media assayed. We will try to correlate the molecular basis of those phenotypes with its transcriptome.

\section{Materials and methods}

\section{Strains and growth conditions}

C. albicans strains, plasmids and bacterial strain used in this study are listed in Table 3.1. Strains were routinely cultured on YEPD or YNB medium at $28^{\circ} \mathrm{C}$. Yeast growth was obtained in YNB medium at $28^{\circ} \mathrm{C}$ supplemented with $0.2 \mathrm{mM}$ of uridine for growth of $U R A 3^{-}$strains. Solid medium was obtained by adding agar (2\%).

Solid media for inducing the yeast-hypha transition in C. albicans were Lee medium in which glucose was replaced by $\mathrm{N}$-acetylglucosamine $(1.25 \%)$, Spider medium (1\% nutrient broth, $1 \%$ mannitol, $0.2 \% \mathrm{~K}_{2} \mathrm{HPO}_{4}$ ), Lee medium plus $4 \%$ bovine calf serum (GIBCO), SLAHD and Embedded medium.

Cells were grown at $28^{\circ} \mathrm{C}$ in YEPD medium, and approximately 50 cells were spread on different agar medium plates. The dimorphic transition in liquid media was induced by growing cells in Lee at $28^{\circ} \mathrm{C}$ and changing them at a density of $10^{7}$ cells $/ \mathrm{ml}$ to $37^{\circ} \mathrm{C}$ in Lee plus $4 \%$ bovine calf serum or Lee medium with $1.25 \% \mathrm{~N}$-acetylglucosamine (GlcNAc)(Sigma-Aldrich) instead of glucose and incubated at $37^{\circ} \mathrm{C}$. Escherichia coli strain was grown at $37^{\circ} \mathrm{C}$ in Luria-Bertani medium (LB) supplemented with $100 \mu \mathrm{g}$ of ampicillin per $\mathrm{ml}$ for plasmid selection (Sambrook, 2001). 
Table 3.1 Candida albicans strains, plasmids and bacterial strain used in this study

\begin{tabular}{|c|c|c|c|}
\hline & Relevant genotype or characteristics & Parent & Reference \\
\hline \multicolumn{4}{|c|}{ C. albicans strains } \\
\hline SC5314 & Clinical isolated (Wild type) & - & (Gillum, 1984) \\
\hline CAl4 & ura3s::imm434/ura34::imm434 & CAF2-1 & (Fonzi and Irwin, 1993) \\
\hline CAMR & ura34::imm434/URA3 & CAI4 & (Rodrigues and Dominguez, 2005) \\
\hline RDG4 & $\begin{array}{l}\text { gen54::hisG /gcn54::hisG } \\
\text { ura34::imm434/ura34::imm434 }\end{array}$ & RDG3 & (Degano and Dominguez, 2005) \\
\hline SFC4 & $\begin{array}{l}\sin 34:: \text { his } G \text { /sin34::hisG } \\
\text { ura34::imm434/ura34::imm434 }\end{array}$ & SFC3 & (Monterola and Dominguez, 2002) \\
\hline ZAH1 & $\begin{array}{l}\text { sin34::hisGURA3hisG/SIN3 } \\
\text { ura34::imm434/ura34::imm434 } \\
\text { gcn54::hisG/gcn54::hisG }\end{array}$ & RDG4 & present work \\
\hline ZAH2 & $\begin{array}{l}\text { sin34:::hisG/SIN3 } \\
\text { ura34::imm434/ura34::imm434 } \\
\text { gcn54::hisG/gcn54::hisG }\end{array}$ & $\mathrm{ZAH} 1$ & present work \\
\hline ZAH3 & 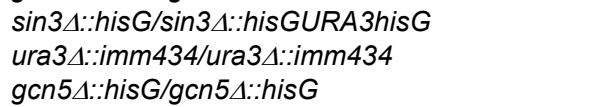 & $\mathrm{ZAH} 2$ & present work \\
\hline ZAH4 & 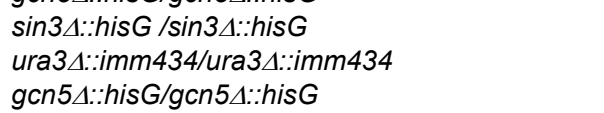 & ZAH3 & present work \\
\hline ZAHR4 & $\begin{array}{l}\sin 3 \Delta:: \text { his } / \sin 3 \Delta:: \text { his } \\
\text { ura34::imm434/URA3 } \\
\text { gcn54::hisG/gcn54::hisG }\end{array}$ & $\mathrm{ZAH} 4$ & present work \\
\hline EVA1 & $\begin{array}{l}\text { ura34::imm434/URA3 } \\
\sin 3 \Delta:: h i s G / \sin 3 \Delta:: \text { hisG }\end{array}$ & SFC4 & present Work \\
\hline \multicolumn{4}{|c|}{ Plasmids } \\
\hline pSNC3 & $\begin{array}{l}\text { Plasmid pBluescript } \mathrm{KS}^{+} \text {containing the } \\
\text { disruption cassette of the SIN } 3 \text { gene }\end{array}$ & pSNC1 & (Monterola, 2002) \\
\hline pGEM-T & $\begin{array}{l}\text { Plasmid containing ampicillin resistance gene, } \\
\text { T7 and SP6 RNA Polymerase transcription } \\
\text { initiation site, T7 and SP6 RNA Polymerase } \\
\text { promoter, multiple cloning site, lacZ start } \\
\text { codon, lac operon sequences, lac operator, } \beta- \\
\text { lactamase coding region, phage f1 region, } \\
\text { binding site of pUC/M13 Forward Sequencing } \\
\text { Primer, binding site of pUC/M13 Reverse } \\
\text { Sequencing Primer }\end{array}$ & & Promega (Robles, 1994) \\
\hline pMR1 & $\begin{array}{l}\text { Plasmid containing the reinsertion cassette of } \\
\text { the URA3 gene }\end{array}$ & & (Rodriguez and Dominguez) \\
\hline \multicolumn{4}{|c|}{ Bacterial strain } \\
\hline DH5a & $\begin{array}{l}F \Phi \text { 80lac Z } 4 M 15, \Delta \text { (lacZYA-argF), U169, } \\
\text { deoR, recA1, endA1, hsdR17, phoA, supE44, } \\
\left(r^{\prime}, k^{+}\right), \text {gyrA96, } \Delta^{-} \text {thi-1, gyrA96relA }\end{array}$ & & (Hanahan and Meselson, 1983) \\
\hline
\end{tabular}

\section{Reagents}

Agar, yeast extract, bactopeptone, yeast nitrogen base, cornmeal agar and glucose were purchased from Difco Laboratories (Detroit, MI. USA); DNA restriction and modification enzymes were purchased from Roche, Invitrogen; Fermentas and Promega, unless otherwise stated. 10x buffers for restriction enzymes were those supplied by manufacturers. For the double digestions, Carlos Buffer (CB 10x), DTT 10× (DTT $10 \mathrm{mM}$ ) and spermidin 10x were used. The usual chemicals were purchased from Sigma Chemical Co. (St. Louis, MO, USA) and Merck (Merck and Co. Inc). 


\section{Strain construction}

To investigate the function of GCN5 and SIN3 in C. albicans, the gene SIN3 was deleted in the gcn5 5 background strain (Degano, Ph.D thesis in process). The plasmid pSNC3 was constructed in our laboratory by ligating the PCR products generated from the up-stream (600 bp) and down-stream (588 bp) flanking regions of CaSIN3 to a hisG-URA3-hisG cassette in pSNC1. The pSNC3 was linearized by Sacl and Kpnl and from the resulting plasmid; a 5.1-kb Sacl-Kpnl fragment was isolated and used to transform strain RDG4 ( $\Delta g c n 5)$. The $C$. albicans strain, heterozygous for the first CaSIN3 disrupted allele, were further selected on the medium containing 5-fluoroorotic acid to get Ura- cells (Fonzi \& Irwin, 1993). The positive clone was utilized for the deletion of the second remaining wild-type allele. The verification of CaSIN3 deletion in the heterozygous and homozygous strains was monitored by PCR and Southern blotting using a Rediprime ${ }^{T m}$ ॥ Random Prime labelling system (AmershamBiosciences). The $588 \mathrm{bp}$ hybridization probe corresponding to down-stream flanking regions of CaSIN3 was used. Chromosomal DNA isolated from all strains was digested with Ndel for Southern blotting.

\section{DNA manipulation}

Restriction analysis, DNA cloning, agarose gel electrophoresis were performed using standard procedures (Sambrook, 2001). Polymerase chain reactions were carried out in an Eppendorf Master Cycler gradient and PERKIN ELMER Gene Amp PCR System 2400.

\section{Spot assay for analyzing sensitivity to different substances on plates}

Methods for testing the $C$. albicans strains were similar for all effectors. Cultures were grown in $50 \mathrm{ml}$ of YEPD medium until the exponential phase and then diluted to an optical density at $600_{\mathrm{nm}}$ of 0.4 . Five microliters of undiluted cell culture and $1 / 10$ serial dilutions of each cell culture were spotted onto YEPD plates containing the following: $\mathrm{NaCl}(0.5$ to $1 \mathrm{M})$, Calcofluor white $(0.1$ to $0.5 \mathrm{mg} / \mathrm{ml})$, Caffeine (8 to $15 \mathrm{mM})$, Sodium dodecyl sulfate (SDS; 0.005 to $0.01 \%$ ), Fluconazole (2 to $4 \mu \mathrm{g} / \mathrm{ml}$ ), Hygromycin (100 to $300 \mu \mathrm{g} / \mathrm{ml}$ ), Amphotericin B (50 to $100 \mu \mathrm{g} / \mathrm{ml}$ ), Itraconazole $(4 \mu \mathrm{g} / \mathrm{ml}), \mathrm{CaCl}_{2}(0.5$ to 1.5 $\mathrm{M}), \mathrm{KCl}(1.5 \mathrm{M})$, and Congo red $(0.1$ to $0.5 \mathrm{mg} / \mathrm{ml})$. Differences in growth were recorded after incubation of the plates at $28^{\circ} \mathrm{C}$ for $96 \mathrm{~h}$. Zymolyase, Alcian blue binding assay and RNA isolation were performed as described in Chapter II. 
cDNA preparation and microarray hybridization also were carried out as described in previous chapter.

\section{Virulence studies}

The mouse strain employed in the infection model was male CD1. Mice were infected through the intra-peritoneal mode with a reconstructed $U R A 3 \sin 3 \Delta$ and $\sin 3 \Delta, g \operatorname{cn} 5 \Delta$ double mutant. The $C$. albicans cells were injected with $10^{8}$ (data not shown) and $5 \times 10^{8}$ cells. The mortality rate was monitored for 30 days.

\section{Results}

\section{Construction of C. albicans gen $5 \Delta, \sin 3 \Delta$ double mutant by gene disruption}

The "Ura blaster" technique was used to sequentially disrupt both copies of the $C$. albicans SIN3 gene in the gcn5D mutant background strain. Chromosomal DNA isolated from wild-type and transformant strains were digested with $\mathrm{Ndel}$ and analyzed by Southern hybridization (Fig. 3.1B). A 588-bp Sall-KpnI DNA fragment derived from plasmid pSNC3-3' was used as a specific probe. The wild-type SIN3 allele showed a 3.6-kb band (Fig. 3.1B, Lane 1 and 3). A new band which represented the "Ura blaster" integrated into one SIN3 allele appeared in the transformants (Fig. 3.1B, Lane 4). After selection on 5-fluoroorotic acid-containing medium, the loss of the URA3 gene and one copy of the hisG element resulted in a 9.8-kb band (Fig. 3.1B, Lane 5). The remaining intact SIN3 allele was disrupted similarly, leading to homozygous sin3::hisG/Asin3::hisG-URA3-hisG strains (Fig. 3.1B, Lane 6) and corresponding Uraderivatives (Fig. 3.1B, Lane 7). Phenotypes reported in this chapter were observed in at least two independently isolated disrupted. Our ability to generate viable gcn $5 \Delta$, $\sin 3 \Delta$ double mutant indicates that both genes are not essential in $C$. albicans.

Table 3.2 Oligonucleotides used in this study

\begin{tabular}{|c|c|c|c|}
\hline Primer & Sequence $5^{\prime}-3^{\prime}$ & Primer & Sequence $5^{\prime}-3^{\prime}$ \\
\hline RD17 & GGTGACAAACTAGTTCAATGC & RD57 & ACTGAAGTAGACTTCCCACT \\
\hline RD18 & GCTTATCAAGCTTTGGAAG & URA1 & GGATACTATCAAACAAGAGG \\
\hline RD19 & CCACTCGAGACGTAATGG & URA2 & AATGCTGGTTGGAATGCTTA \\
\hline RD24 & GCGGCCGCCACAAGAAT & AMH1 & CCGAACATCAACCAATCGT \\
\hline RD32 & GGCAATCACATCAAGACT & IP2 & TTACAATCAAAGGTGGTCC \\
\hline RD33 & TGTTCAACTTCAAGTCTC & IP3 & GGTACAGTTGTTCCTCACA \\
\hline RD50 & GGTCAAGGTACCAACAGATT & SINCA10 & GGTTCAACATCCCATCGATCA \\
\hline RD51 & CATCGCTAGTAGTTCTGACT & SINCA15ter & CATGGCAATCTGTTTCATCAA \\
\hline RD54 & ATTGAAAGACTGCAGTTTTGT & SINCA15orf & AATGGAACACAGTTGCCG \\
\hline RD55 & CTTATTCTCGAGCAATGCTT & SINCA16 & TCTCTGTCATGTACTGAG \\
\hline RD56 & TATCCATTGTCCAGGTGGTT & & \\
\hline
\end{tabular}




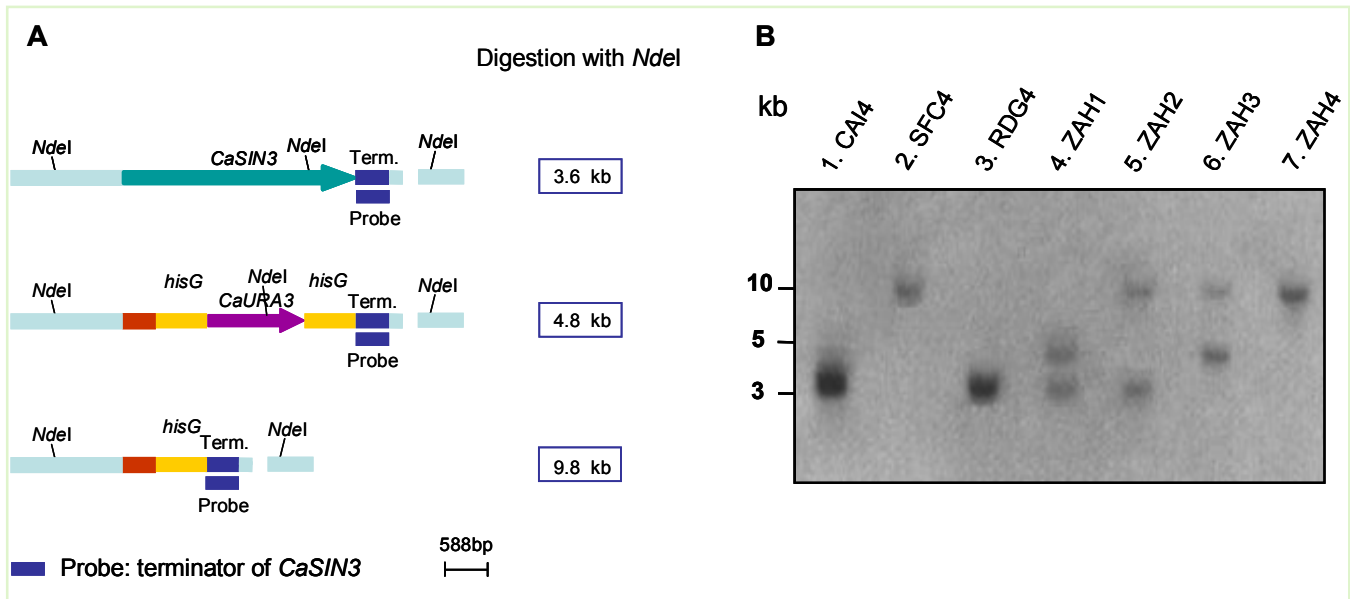

Figure 3.1 Deletion of CaSIN3 alleles. (A) Structure of different alleles. The wild-type SIN3 gene and the alleles disrupted by the hisG-URA3-hisG cassette or by hisG alone are shown. (B) Southern blot analysis of genomic DNA was performed with the following strains digested with Ndel.

Lane 1, CAI4 (GCN5/GCN5; SIN3/SIN3)

Lane 2, SFC4 (GCN5/GCN5; $\sin 34::$ hisG/sin34::hisG)

Lane 3, RDG4 (gcn54::hisG/gcn54::hisG; SIN3/SIN3)

Lane 4, ZAH1 (gcn54::hisG/gcn54::hisG; SIN3/sin34:hisGURA3hisG)

Lane 5, ZAH2 (gcn54::hisG/gcn54::hisG; SIN3/sin34::hisG)

Lane 6, ZAH3 (gcn54::hisG/gcn54::hisG; $\sin 34::$ hisGURA3hisG/sin34::hisG)

Lane 7, ZAH4 (gcn54::hisG/gcn54::hisG; $\sin 34:: h i s G / \sin 34:: h i s G)$

The $588 \mathrm{bp}$ fragment was used as a probe.

Growth curves of the CAI4, both single and double mutants showed difference in beginning of the exponential growth phase in either YEPD (Fig. 3.2) or YNB medium. The beginning of the exponential growth phase in the double mutant was delayed by up to $18 \mathrm{~h}$. The growth phenotype observed in the $C$. albicans gcn5 5 , $\sin 3 \Delta$ mutant is not as severe as the defect displayed by the gcn5 $\Delta$ mutant that grows at a rate about one-half that of the CAI4 (Degano, Ph.D thesis in process). Since the growth defect of gcn5 mutant was slightly suppressed by deletion of the SIN3 gene. Our results suggest that the deletion of SIN3 in a gcn5 $\Delta$ background mutant slightly compensate the growth defect of the gcn $5 \Delta$ mutant.

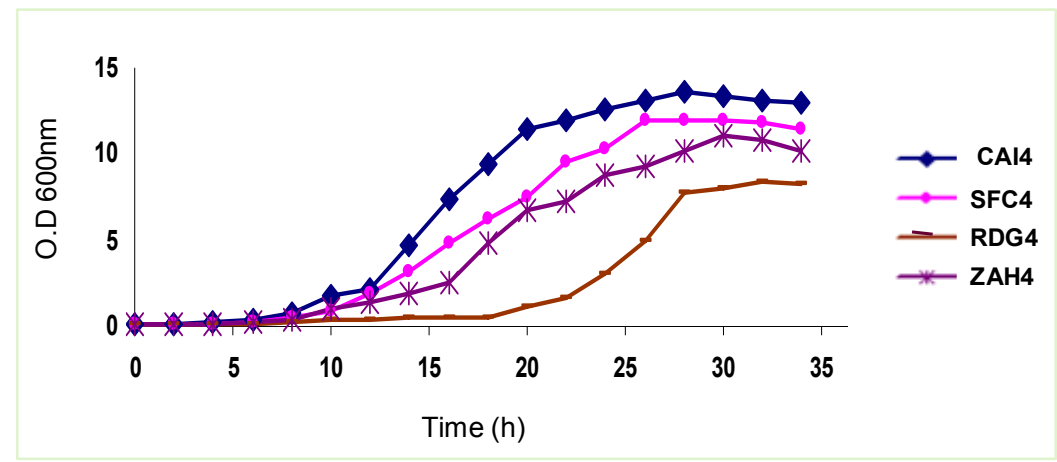

Figure 3.2 Growth curves of CAI4, RDG4 $(g c n 5 \Delta)$, SFC4 $(\sin 3 \Delta)$ and ZAH4 $(g c n 5 \Delta, \sin 3 \Delta)$. Strains were pre-grown on rich medium containing $1 \%$ glucose and inoculated at an O.D at $600 \mathrm{~nm}$ of 0.1 YEPD. Growth was monitored over a period of $34 \mathrm{~h}$ by measuring the optical density of the cultures. 
Morphologically, the double mutant likes both simple mutants are very different to CAI4 strain as show in Fig. 3.3.

The culture of the $g c n 5 \Delta, \sin 3 \Delta$ double mutant in YEPD liquid medium was composed of a mixture of rode or cigarette shape cells and short chains of elongated, aberrantly shaped cells attached to one another, suggesting an intermediate phenotype between the one displayed by the $g c n 5 \Delta$ and $\sin 3 \Delta$ mutants.

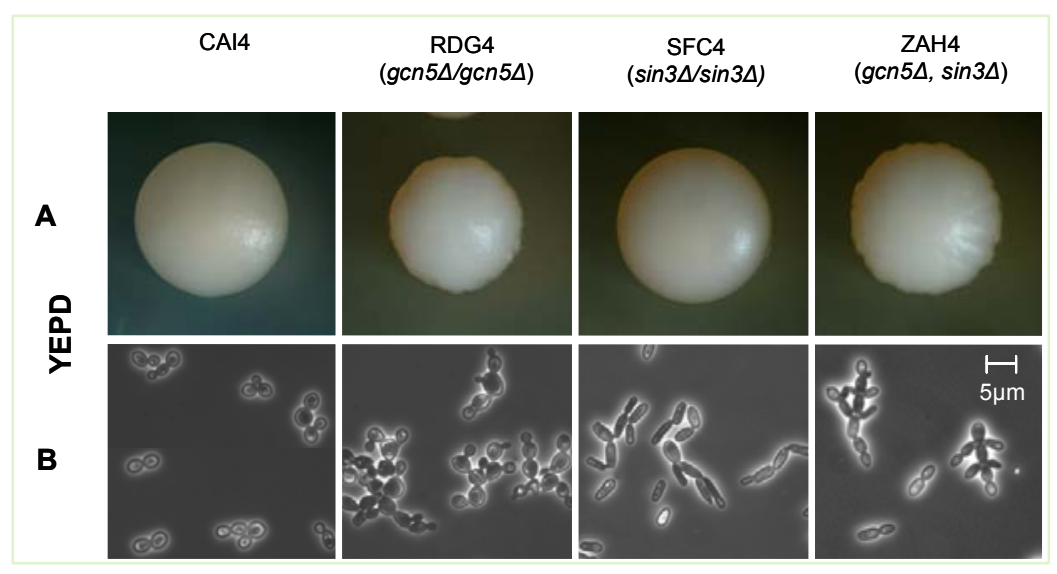

Figure 3.3 (A) Colony growth and (B) morphological characteristics of $C$. albicans homozygous strains CAI4, RDG4, SFC4 and ZAH4 in YEPD medium.

\section{Behavior of the $g c n 5 \Delta, \sin 3 \Delta$ double mutant in solid media}

In order to determine whether the absence of $C$. albicans $G c n 5 p$ and $\operatorname{Sin} 3 p$ is involved in hyphal morphogenesis, we examined the ability of CAI4, double and both single mutants to undergo hyphal transition under several conditions on solid media. Cells were grown at $28^{\circ} \mathrm{C}$ in YEPD medium and approximately 50 cells were spread on different agar media plates containing Spider, Lee plus GIcNAc, and Lee plus 4\% bovine calf serum (GIBCO), SLAHD and Embedded medium. We found that the homozygous mutant lacked the ability to form lateral hypha at $37^{\circ} \mathrm{C}$ on all solid media, even in the presence of serum, a strong inducer of the dimorphic transition. The appearance of colonies after 4 days of growth at $37^{\circ} \mathrm{C}$ in all solid media can be seen in 


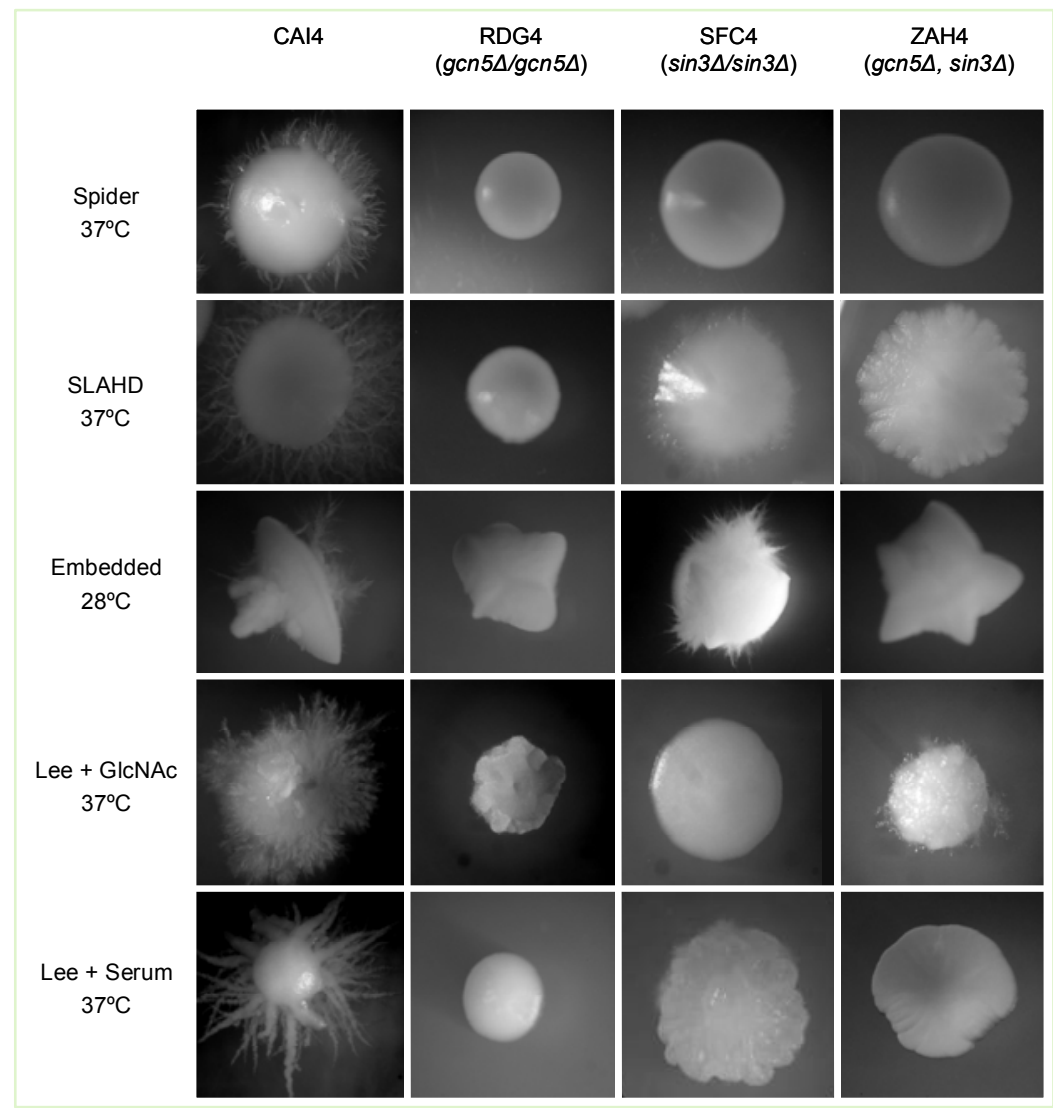

Figure 3.4 Colony growth of the $g c n 5 \Delta, \sin 3 \Delta$ double mutant in solid media. All mutants and CAI4 were grown on solid media which induce hyphal development. The plates were incubated for 4 days at $37^{\circ} \mathrm{C}$.

\section{Behavior of the $g c n 5 \Delta, \sin 3 \Delta$ double mutant in liquid media}

The behavior of the double mutant strain was next investigated by assessing hyphal formation in Lee $\left(\mathrm{pH} 6.8,37^{\circ} \mathrm{C}\right)$ (data not shown) or in serum inducing media.

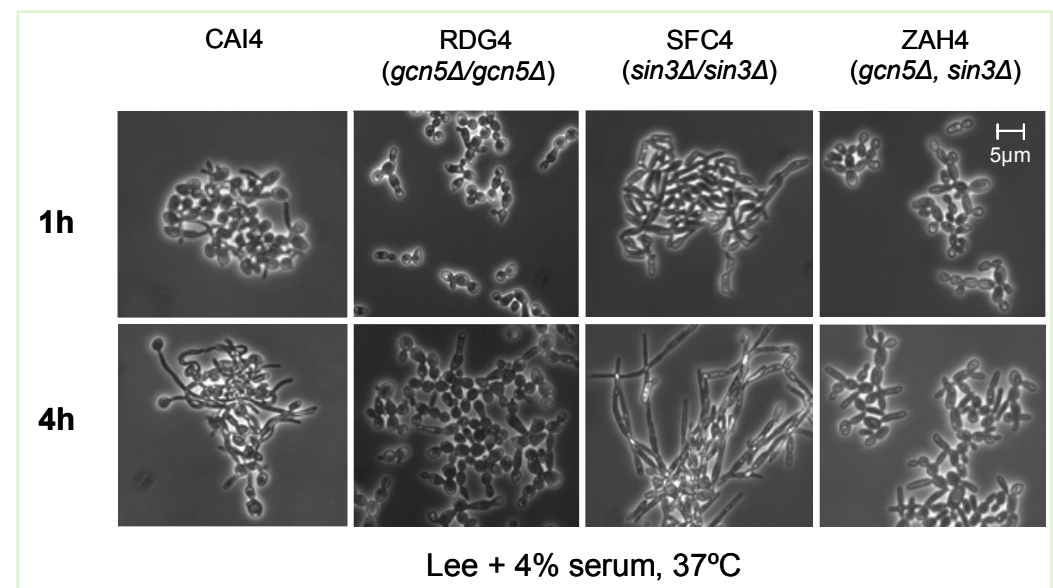

Figure 3.5 Cellular morphology of CAI4, RDG4, SFC4 and ZAH4 strains during the yeast-hypha transition in Lee medium supplemented with $4 \%$ serum at $37^{\circ} \mathrm{C}$ after 1 and 4 hours. 
shows that the double $g c n 5 \Delta, \sin 3 \Delta$ mutant like both simple mutants failed to form germ tubes in the liquid media assayed, indicating that one or more of the pathways involved in the yeast-to-hypha transition didn't function in the double and both single mutants.

\section{Chlamydospore formation}

We attempted to determine whether the deletion of both genes might be involved in the formation of chlamydospores. Therefore, a CAI4 strain, the double and both simple mutants was streaked out lightly on cornmeal agar (Difco)- $0.33 \%$ Tween 80 , covered by cover slips, and incubated at $25^{\circ} \mathrm{C}$ for 7 to 14 days.

The results are shown in Fig. 3.6. The CAI4 strain was able to form chlamydospores. Chlamydospores formed as terminal thick-walled cells on short side branches (suspensor cells) or as terminal cells on filaments. But the double mutant like both simple mutants was completely deficient in chlamydospore formation. These results demonstrate that both proteins are needed in chlamydospore formation.

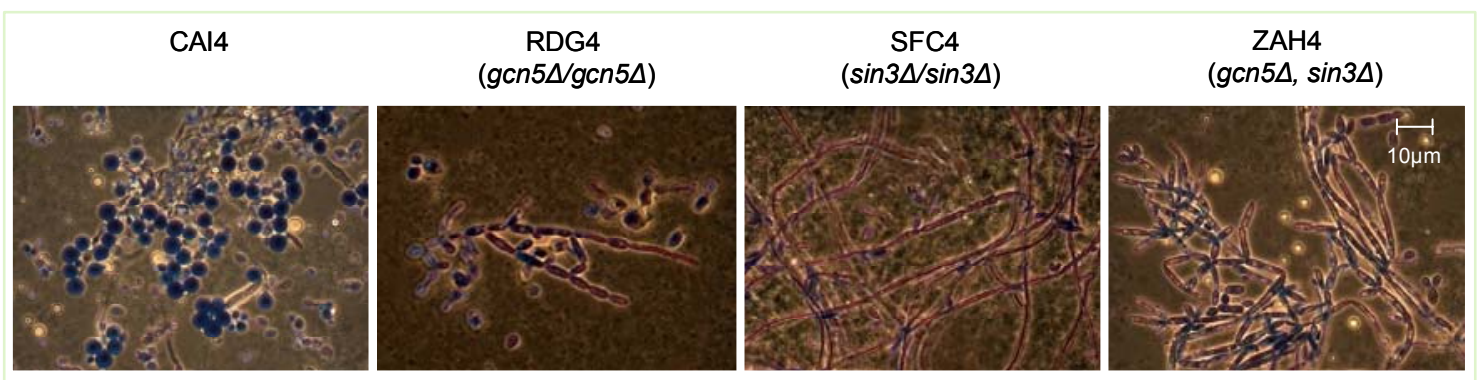

Figure 3.6 Chlamydospore formation of $C$. albicans in the $g c n 5 \Delta, \sin 3 \Delta$ double mutant

\section{Phenotype analysis of the gen $5 \Delta$, sin $3 \Delta$ double mutant}

Possible defects in the cell wall of double mutant were first investigated by experiments including sensitivity to different effectors, using a screening method designed to identify genes involved in cell surface assembly (Lussier, 1997).

We found that the double mutant showed sensitive to Caffeine, Congo red, $\mathrm{NaCl}, \mathrm{KCl}$, Fluconazole, Itraconazole and Hygromycin. No difference in sensitivity was found towards Calcoflour white and SDS when compared to CAI4 strain (Fig. 3.7). 

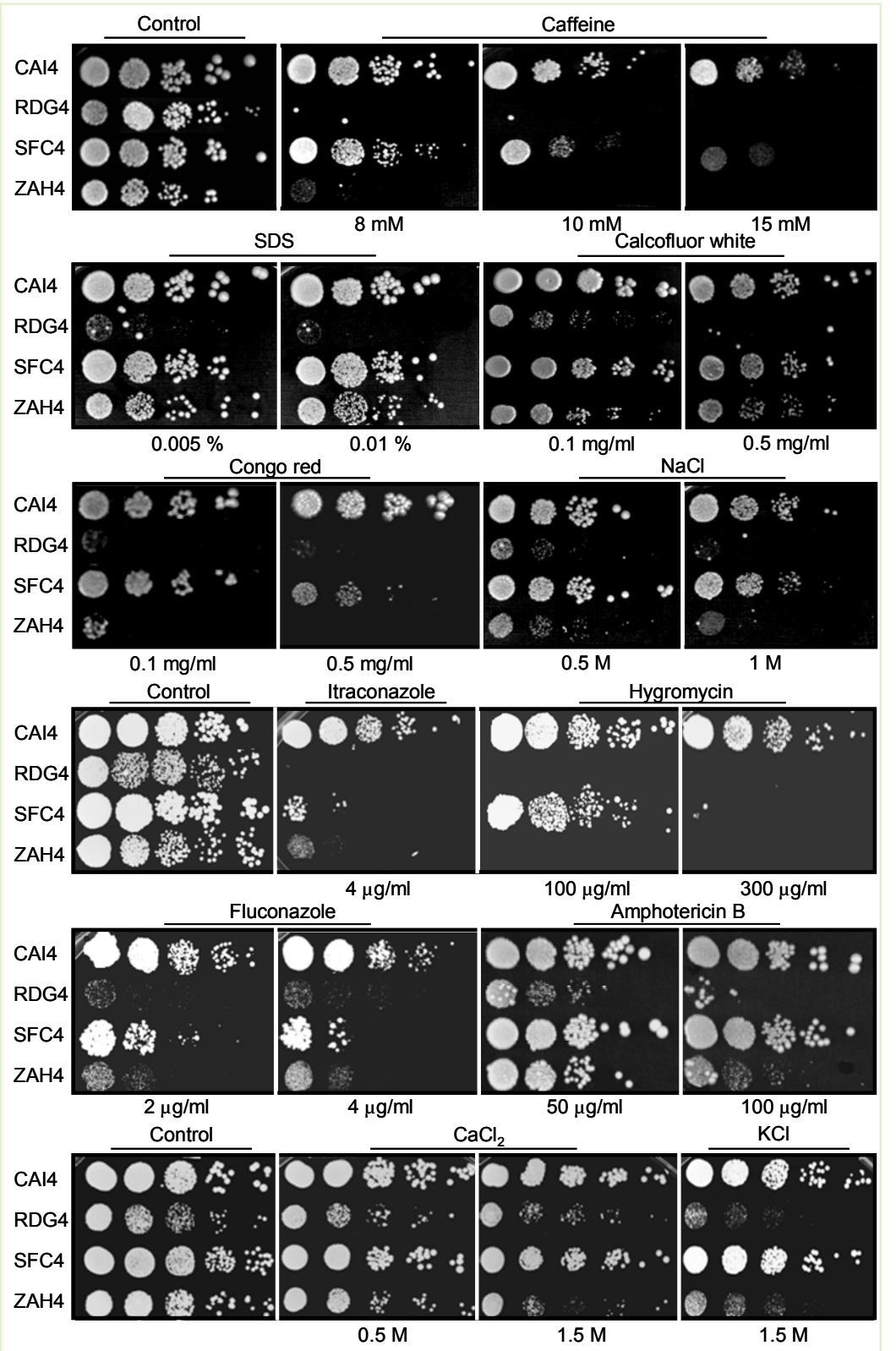

Figure 3.7 Sensibility assays of CAI4, RDG4 $(\Delta g c n 5)$, SFC4 $(\Delta \sin 3)$ and ZAH4 $(g c n 5 \Delta, \sin 3 \Delta)$ to different effectors. All strains were grown in liquid YEPD. The optical density at $600 \mathrm{~nm}$ of the cultures were adjusted to 0.4 with the same medium and $5 \mu$ l aliquots from the cultures and from 10 -fold serial dilutions were spotted onto YEPD plates supplemented with different agents. Plates were incubated at $28^{\circ} \mathrm{C}$ for 4 days.

The phenotype observed in the double and $\sin 3 \Delta$ single mutant is not as severe as the defect displayed by C. albicans gcn5 $\Delta$ mutant, suggesting that Gcn5p is required to maintain normal cell physiology and deletion of SIN3 compensate sensitivity of gcn5 mutant in Calcoflour white and SDS. The sensitivity of yeast cells to Zymolyase has been carried out to uncover changes in cell wall composition and arrangement (Ram et al., 1994). Our results indicated that there were no significant differences between the double, $\sin 3 \Delta$ simple mutants and the CAI4 strain as regards sensitivity to Zymolyase (Fig. 3.8). 


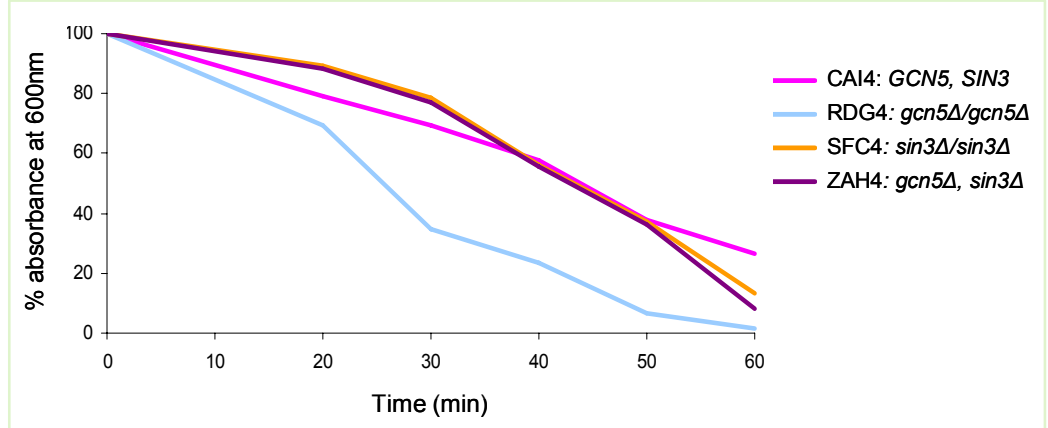

Figure 3.8 Resistance to a cell wall-degrading enzymatic complex of C. albicans. All strains were grown until the exponential phase in YEPD at $28^{\circ} \mathrm{C}$. Aliquots $\left(2 \times 10^{7}\right.$ cells $)$ were resuspended in $10 \mathrm{mM}$ Tris- $\mathrm{Hcl} \mathrm{pH} 7.5$ and treated with $10 \mu \mathrm{g}$ of Zymolyase per ml. The decrease in optical density (O.D) (percentage of resistant cells) is monitored for $60 \mathrm{~min}$.

\section{Alcian blue binding assay}

We then determined the level of Alcian blue binding assay of CAI4 and three mutant strains of $C$. albicans, using the method previously described by Herrero et al (Herrero et al., 2002). Both double and $\sin 3 \Delta$ simple mutants showed a significant decrease in Alcian blue binding suggesting a modification of the cell wall composition (Fig. 3.9).

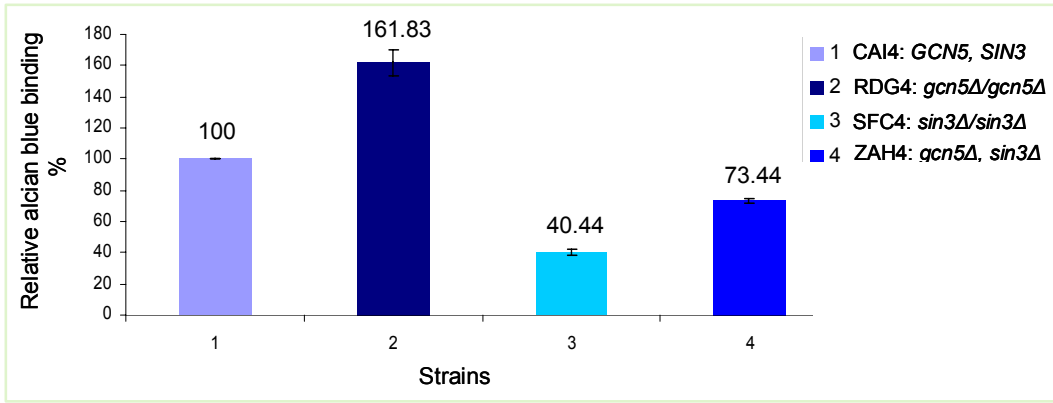

Figure 3.9 Cell surface charges in C. albicans strains. Alcian blue binding assays. Relative dye binding was calculated as the percentage of dye bound compared with results for the parental strains (CAI4, RDG4 and SFC4). Results are average of two independent determinations; bars indicate standard deviations.

\section{Virulence assay}

To test whether $C$. albicans $G c n 5 p$ and $\operatorname{Sin} 3 p$ influence virulence in systemic animal models, $10^{8}$ (Data not show) and $5 \times 10^{8}$ cells of a reconstructed URA3 in the $\sin 3 \triangle$ and $g c n 5 \Delta, \sin 3 \Delta$ double mutants and CAI4 strain were injected intra-peritoneally of CD1 mice and monitored mouse survival for a period of 30 days.

Two days after infection, all the mice injected with the CAMR strain, showed signs of systemic disease, including weight loss (Data not shown) and rapid mortality. In contrast, $80 \%$ of mice injected with the double, $\sin 3 \Delta$ and gcn5 $\Delta$ (Rashki, Ph.D thesis 2009) simple mutants survived for this observed period (Fig. 3.10). 


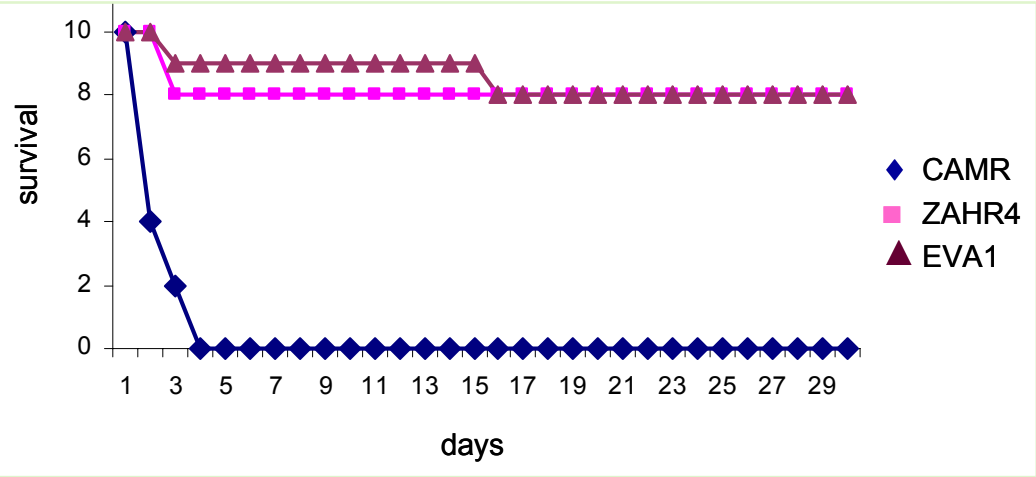

Figure 3.10 Virulence assays. Survival curves for mice $(n=10$ for each $C$. albicans strain at each inoculation dose) infected with $5 \times 10^{8}$ cells of $C$. albicans. CAMR (CAI4-URA $A^{+}$, = ZAHR4 $(g c n 5 \Delta, \sin 3 \Delta-$ $\left.U R A^{+}\right)$and $\triangle E V A 1\left(\sin 3 \Delta-U R A^{+}\right)$.

\section{Global transcriptional responses of the C. albicans gcn5 $\sin 3 \Delta$ double mutant}

A transcriptional profiling analysis was performed to further examine the roles of Gcn5p and $\operatorname{Sin} 3 p$ proteins in $C$. albicans. The transcriptome of the $g c n 5 \Delta, \sin 3 \Delta$ double mutant was compared with the CAI4 strain during the yeast mode of growth and during the yeast-hypha transition. Previously, in our laboratory we had performed experiments in which the transcriptomes of the gcn $5 \Delta$ and $\sin 3 \Delta$ simple mutants were analyzed. Genes that displayed reproductive and statistically significant changes in expression were identified under both yeast growth conditions and during the yeast-hypha switch. A list of differentially expressed genes in the double mutant is provided as supplementary data (Supplementary tables 3.1-3.4). To explore the functions of the $g c n 5 \Delta, \sin 3 \Delta$ double mutant under condition that promoted growth in the yeast form, we compared the transcriptomes of CAI4 and the double mutant strain in YNB medium at $28^{\circ} \mathrm{C}$. In the $g c n 5 \Delta, \sin 3 \Delta$ double mutant we detected an altered expression of 558 genes by $\geq 1.5$-fold: 294 genes being up-regulated and 264 down-regulated. When transcriptome of cells growing in Lee medium at $37^{\circ} \mathrm{C}$ during the hypha transition were compared, 1271 genes modified their expression level, including 690 up- and 581 down-regulated genes. Approximately $30 \%$ of the $C$. albicans genes showed significant changes in their expression levels $(\rho$-value $\leq 0.02)$, indicating a global transcriptional response.

\section{Differential gene expression in exponential growth phase}

The analysis of the microarray data of cells in the exponential growth phase showed 558 affected genes (Supplementary tables 3.1 and 3.2). Among the total number of altered genes, 131 (23.47\%) were genes with unknown functions. Amongst the rest of the genes, HSP12, encode a heat shock protein with homology to the $S$. cerevisiae 
HSP12, showed the highest fold change $(6.40$-fold), whereas the lowest expression level found in this experiment was observed for IPF191 (-5.88 -fold), which encodes a putative transporter. Due to the large number of genes found to be up- or downregulated, this analysis was focussed on a few of the representated functional categories.

\section{Genes involved in DNA synthesis and replication}

Our microarray analysis indicated that thirteen genes involved in DNA synthesis and replication e.g. CDC21, DPB2, HYS2, POL2, POL3, RAD6 and TOP1 were upregulated (Table 3.3). In addition, two genes -RFA1 and RFA2- that function as part of DNA replication factor also were up-regulated. It has been described that overexpression of RFA1 may functions in the cell cycle delay at G2/M phases by disrupting spindle attachment to chromosomes and activating the DNA damage checkpoint (Niu et al., 2008), which may be responsible for the elongated cell in the double mutant. Upregulation of RFA1 in the $\sin 3 \triangle$ simple mutant has been reported (Martin, Ph.D thesis 2006 ) suggesting that $\operatorname{Sin} 3 p$ may play a role in the regulation of cell cycle progression. In agreement with our data, expression of genes playing a role in the G2 cell cycle progression in SIN3-deficient cells has also been reported in Drosophila (Pile et al., 2003). Twelve genes encoding for oxidative stress including AHP2 (Fernandez-Arenas et al., 2007), GAD1 (Bensen et al., 2004), GLO1 (Wang et al., 2006), GLR1 (Alarco \& Raymond, 1999; Fradin et al., 2005; Wang et al., 2006), GRE3 (Ramsdale et al., 2008), GRP2 (Enjalbert et al., 2006), GRP6, GRX3 (Yin et al., 2004), MCR1 (Bensen et al., 2004) SOD2, TRX1 and TSA1 (Seneviratne et al., 2008) were found to be up-regulated and three of them, AHP2, MCR 1 and TSA1 overlapped with the $\sin 3 \triangle$ simple mutant.

Table 3.3 Differentially expressed genes in $g c n 5 \Delta, \sin 3 \Delta$ mutant during the yeast growth phase

\begin{tabular}{lclc}
\hline Gene name & ORF & Description & $\begin{array}{c}\text { Fold } \\
\text { regulation }\end{array}$ \\
\hline DNA synthesis and replication & & & \\
CDC21 & orf19.3549 & DNA polyamerase III & 1.59 \\
DBP3 & orf19.12334 & DNA -directed DNA polymerase epsilon subunit B & 1.76 \\
HYS2 & orf19.3960 & DNA-directed DNA polymerase delta & 2.01 \\
POL2 & orf19.2365 & DNA-directed DNA polymerase epsilon, & 1.74 \\
POL3 & orf6.3494 & DNA polymerase III & 1.88 \\
RAD51 & orf19.3752 & DNA repair protein & 2.16 \\
RAD6 & orf19.7195 & Ubiquitin protein ligase & 1.71 \\
RFA1 & orf19.2093 & DNA replication factor & 1.62 \\
RFA2 & orf19.2267 & DNA replication factor A & 2.36 \\
RNR1 & orf19.5779 & Ribonucleoside-diphosphate reductase & 2.94 \\
RNR21 & orf19.5801 & Ribonucleoside-diphosphate reductase & 1.77 \\
RNR22 & orf19.1868 & Ribonucleoside-diphosphate reductase & 3.03 \\
TOP1 & orf19.7742 & DNA topoisomerase I & 2.58 \\
Oxidative stress & response & & \\
AHP2 & orf19.6470 & Macrophage-downregulated gene & \\
GAD1 & orf19.1153 & Glutamate decarboxylase & 2.37 \\
GLO1 & orf19.6058 & Oxidative stress-induced via Cap1p & 1.02 \\
\hline
\end{tabular}




\begin{tabular}{|c|c|c|c|}
\hline GLR1 & orf19.11623 & Glutathione reductase; oxidative stress-induced via Cap1p & 1.73 \\
\hline GRE3 & orf19.4317 & D-xylose reductases & 1.78 \\
\hline GRP2 & orf19.4309 & $\begin{array}{l}\text { Similar to } S \text {. cerevisiae Gre } 2 p \text { (methylglyoxal reductase); } \\
\text { induced in core stress response or by oxidative stress }\end{array}$ & 5.54 \\
\hline GRP6 & orf19.3151 & Putative reductase & 1.84 \\
\hline GRX3 & orf19.2727 & Glutaredoxin-like protein & 2.25 \\
\hline MCR1 & orf19.3507 & Protein described as NADH-cytochrome-b5 reductase & 2.33 \\
\hline SOD2 & orf19.3340 & Mitochondrial manganese-containing superoxide dismutase & 1.81 \\
\hline TRX1 & orf19.7611 & Thioredoxin & 1.79 \\
\hline TSA1 & orf19.7417 & $\begin{array}{l}\text { Protein of TSA/alkyl hydroperoxide peroxidase C (AhPC) } \\
\text { family }\end{array}$ & 1.92 \\
\hline \multicolumn{4}{|c|}{ 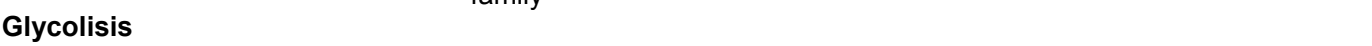 } \\
\hline ALD5 & orf19.13228 & Protein described as an aldehyde dehydrogenase & 3.10 \\
\hline FBA1 & orf19.4618 & $\begin{array}{l}\text { Putative fructose-bisphosphate aldolase; enzyme of } \\
\text { glycolysis }\end{array}$ & 2.02 \\
\hline FBP1 & orf19.6178 & Fructose-1,6-bisphosphatase & 1.72 \\
\hline PCK1 & orf19.7514 & Phosphoenolpyruvate carboxykinase & 4.15 \\
\hline \multicolumn{4}{|c|}{ Oxidation of fatty acids } \\
\hline $\mathrm{ECl} 1$ & orf19.6445 & $\begin{array}{l}\text { Protein similar to } S \text {. cerevisiae Eci } 1 p \text {, which is involved in } \\
\text { fatty acid oxidation }\end{array}$ & 1.99 \\
\hline POT1 & orf19.7520 & Putative peroxisomal 3-oxoacyl CoA thiolase & 1.94 \\
\hline POX1-3 & orf19.9221 & Predicted acyl-CoA oxidase; farnesol regulated & 1.83 \\
\hline SPS20 & orf19.4157 & Peroxisomal 2,4-dienoyl-CoA reductase & 1.96 \\
\hline \multicolumn{4}{|c|}{ Siderophore-iron transport } \\
\hline FET33 & orf19.943 & Putative multicopper ferro- $\mathrm{O}_{2}$-oxidoreductase & 1.70 \\
\hline FRE10 & orf19.1415 & Major cell-surface ferric reductase under low-iron conditions & 1.97 \\
\hline FTR1 & orf19.7219 & High-affinity iron permease & 3.56 \\
\hline FTR2 & orf19.7231 & High-affinity iron permease; & 2.22 \\
\hline \multicolumn{4}{|c|}{ Pentose-phosphate pathway } \\
\hline PRS1 & orf19.969 & Ribose-phosphate pyrophosphokinase & 2.24 \\
\hline TKL1 & orf19.5112 & Putative transketolase & 1.87 \\
\hline ZWF1 & orf19.12218 & Putative glucose-6-phosphate dehydrogenase & 1.79 \\
\hline \multicolumn{4}{|c|}{ Chitin anabolism } \\
\hline CHS3 & orf19.4937 & Major chitin synthase of yeast-form and hyphal cells & 1.91 \\
\hline CHT2 & orf19.3895 & Chitinase; putative N-terminal catalytic domain & 2.17 \\
\hline CHT3 & orf19.7586 & Chitinase & 2.34 \\
\hline \multicolumn{4}{|c|}{ Nuclear migration } \\
\hline NUM11 & orf19.4715 & Nuclear migration protein & 1.92 \\
\hline NUM12 & orf19.2924 & Nuclear migration protein & 2.21 \\
\hline TUB1 & orf19.7308 & Alpha-tubulin & 1.73 \\
\hline TUB2 & orf19.6034 & Beta-tubulin & 1.58 \\
\hline \multicolumn{4}{|c|}{ Tricarboxylic-acid pathway } \\
\hline FUM12 & orf19.6725 & $\begin{array}{l}\text { Protein described as similar to fumarate hydratase, enzyme } \\
\text { of citric acid cycle }\end{array}$ & 1.76 \\
\hline IDP2 & orf19.3733 & Putative isocitrate dehydrogenase & 2.19 \\
\hline LSC2 & orf19.710 & Protein described as beta subunit of succinate-CoA ligase & 2.03 \\
\hline MDH1 & orf19.7481 & Protein described as malate dehydrogenase & 1.77 \\
\hline \multicolumn{4}{|c|}{ Glucose transporter } \\
\hline HGT6 & orf19.2020 & $\begin{array}{l}\text { Putative glucose transporter of the major facilitator } \\
\text { superfamily }\end{array}$ & 2.43 \\
\hline HGT8 & orf19.2021 & $\begin{array}{l}\text { Putative glucose transporter of the major facilitator } \\
\text { superfamily }\end{array}$ & 2.11 \\
\hline SHA3 & orf19.3669 & $\begin{array}{l}\text { Protein similar to } S \text {. cerevisiae Sha3p, which is a } \\
\text { serine/threonine kinase involved in glucose transport }\end{array}$ & 1.69 \\
\hline \multicolumn{4}{|c|}{ Cell wall } \\
\hline PGA14 & orf19.968 & Putative GPI-anchored protein of unknown function & 2.69 \\
\hline PGA23 & orf19.11225 & Putative GPI-anchored protein of unknown function & 1.76 \\
\hline PGA4 & orf19.4035 & $\begin{array}{l}\text { Cell-surface protein; predicted glycosylphosphatidylinositol } \\
\text { (GPI) lipid anchor }\end{array}$ & 2.75 \\
\hline PGA54 & orf19.2685 & Putative GPI-anchored protein; hyphal induced & 2.10 \\
\hline PGA56 & orf19.1105.2 & putative GPI-anchor & \\
\hline PMI1 & orf19.8968 & Phosphomannose isomerase; cell wall biosynthesis enzyme & 1.81 \\
\hline PMT2 & orf19.6812 & $\begin{array}{l}\text { Essential protein mannosyltransferase with roles in hyphal } \\
\text { growth }\end{array}$ & 1.77 \\
\hline \multicolumn{4}{|c|}{ Transcription factor } \\
\hline BDF1 & orf19.978 & Putative transcription factor & 2.04 \\
\hline TYE7 & orf19.4941 & $\begin{array}{l}\text { Putative bHLH (basic region, helix-loop-helix) transcription } \\
\text { factor }\end{array}$ & 1.84 \\
\hline ZCF1 & orf19.255 & Predicted zinc-cluster protein of unknown function & 1.95 \\
\hline ZPR1 & orf19.3300 & Protein with putative zinc finger; regulated by Gcn4p & 1.58 \\
\hline
\end{tabular}




\begin{tabular}{|c|c|c|c|}
\hline \multicolumn{4}{|c|}{$\begin{array}{l}\text { Down-regulated genes } \\
\text { Cell wall }\end{array}$} \\
\hline ALS1 & orf19.5741 & Adhesion; ALS family of cell-surface glycoproteins & 0.54 \\
\hline ALS2 & orf19.2122 & ALS family protein which includes cell-surface glycoproteins & 0.61 \\
\hline ALS3 & orf19.2355 & Agglutinin like protein & 0.42 \\
\hline ALS4 & orf19.4556 & $\begin{array}{l}\text { ALS family protein; role in adhesion and wild-type germ tube } \\
\text { induction }\end{array}$ & 0.19 \\
\hline ALS9 & orf19.5742 & $\begin{array}{l}\text { ALS family protein; expressed during infection of human } \\
\text { epithelial cells }\end{array}$ & 0.47 \\
\hline BGL22 & orf19.7339 & Putative glucanase; induced during cell wall regeneration & 0.54 \\
\hline CHS7 & orf19.2444 & Protein required for wild-type chitin synthase III activity & 0.51 \\
\hline CWH41 & orf19.4421 & Processing alpha glucosidase I & 0.42 \\
\hline EAP1 & orf19.1401 & $\begin{array}{l}\text { Cell wall adhesin required for cell-cell adhesion and biofilm } \\
\text { formation }\end{array}$ & 0.29 \\
\hline ECE1 & orf19.3374 & $\begin{array}{l}\text { hyphal-specific expression increases with extent of } \\
\text { elongation of the cell }\end{array}$ & 0.24 \\
\hline PGA16 & orf19.8468 & Putative GPI-anchored protein of unknown function & 0.36 \\
\hline SCW1 & orf19.9345 & Cell surface manno-protein & 0.60 \\
\hline \multicolumn{4}{|c|}{ Electron transport } \\
\hline ATP1 & orf19.6854 & Protein similar to alpha subunit of ATP synthase & 0.43 \\
\hline ATP2 & orf19.5653 & $\begin{array}{l}\text { Protein described as a similar to F1 beta subunit of F1F0 } \\
\text { ATPase complex }\end{array}$ & 0.54 \\
\hline ATP6 & & $\begin{array}{l}\text { Subunit } 6 \text { of the F0 sector of mitochondrial F1F0 ATP } \\
\text { synthase }\end{array}$ & 0.21 \\
\hline $\mathrm{COB}$ & & Cytochrome b & 0.29 \\
\hline COX3A & & Subunit III of cytochrome c oxidase & 0.37 \\
\hline COX3B & & Subunit III of cytochrome c oxidase & 0.46 \\
\hline CYC1 & orf19.1770 & Cytochrome c & 0.49 \\
\hline PET117 & orf19.6225.1 & Cytochrome c oxidase assembly factor & 0.60 \\
\hline PMA1 & orf19.5383 & Plasma membrane $\mathrm{H}(+)$-ATPase & 0.35 \\
\hline \multicolumn{4}{|l|}{ Transport } \\
\hline BET5 & orf19.302 & Targeting and fusion of ER to Golgi transport vesicles & 0.56 \\
\hline FCY24 & orf19.7331 & Putative transporter; more similar to $S$. cerevisiae $T p n 1 p$ & 0.54 \\
\hline HAK1 & orf19.6249 & Putative potassium transporter & 0.31 \\
\hline HOL2 & orf19.4889 & Predicted membrane transporter & 0.22 \\
\hline HOL4 & orf19.12021 & Protein described as an ion transporter & 0.37 \\
\hline IFC1 & orf19.11233 & Oligopeptide transporter & 0.41 \\
\hline IFC3 & orf19.3749 & Oligopeptide transporter & 0.48 \\
\hline IPF1471 & orf19.304 & Putative transporter similar to MDR proteins & 0.43 \\
\hline IPF1524 & orf19.341 & Putative spermidine export pump & 0.62 \\
\hline IPF191 & orf19.3232 & Putative transporter & 0.17 \\
\hline IPF9079 & orf19.4550 & Predicted membrane transporter & 0.59 \\
\hline SSU1 & orf19.7313 & Protein similar to S. cerevisiae Ssu1p sulfite transport & 0.56 \\
\hline \multicolumn{4}{|c|}{ Ribosomal proteins } \\
\hline MRPL27 & orf19.3064 & Putative ribosomal protein & 0.50 \\
\hline MRPL6 & orf19.7486 & Putative mitochondrial ribosomal protein & 0.56 \\
\hline MRS4 & orf19.2178 & $\begin{array}{l}\text { RNA splicing protein and member of the mitochondrial } \\
\text { carrier family (MCF) }\end{array}$ & 0.52 \\
\hline PRP18 & orf19.2112 & U5 snRNA-associated protein & 0.20 \\
\hline PRP31 & orf19.1296 & Pre-mRNA splicing protein & 0.37 \\
\hline $\begin{array}{l}\text { PRP5 } \\
\text { RPL14B }\end{array}$ & $\begin{array}{l}\text { orf19.6831 } \\
\text { orf19.4931.1 }\end{array}$ & $\begin{array}{l}\text { Protein described as a pre-mRNA processing RNA-helicase } \\
\text { Ribosomal protein L14B (by homology) }\end{array}$ & $\begin{array}{l}0.60 \\
0.52\end{array}$ \\
\hline RPL18 & orf19.5982 & Predicted ribosomal protein; & 0.56 \\
\hline RPS620A & orf19.6300 & $\begin{array}{l}\text { Downregulation correlates with clinical development of } \\
\text { fluconazole resistance }\end{array}$ & 0.52 \\
\hline RPS620B & orf19.6301 & Clade-associated gene expression & 0.54 \\
\hline \multicolumn{4}{|c|}{ Transcription factors } \\
\hline GLN3 & orf19.11393 & $\begin{array}{l}\text { GATA transcription factor involved in regulation of } \\
\text { filamentous growth }\end{array}$ & 0.51 \\
\hline HAC1 & orf19.2432 & $\begin{array}{l}\text { Putative transcription factor involved in unfolded protein } \\
\text { response }\end{array}$ & 0.64 \\
\hline HAP2 & orf19.8814 & $\begin{array}{l}\text { CCAAT-binding factor regulates low-iron (chelation) } \\
\text { induction of FRP1 }\end{array}$ & 0.61 \\
\hline IPF1674 & orf19.6559 & Putative transcription initiation factor & 0.54 \\
\hline IPF1731 & orf19.3088 & Putative transcription factor with bZIP DNA-binding motif & 0.49 \\
\hline IPF29 & orf19.5975 & $\begin{array}{l}\text { Putative transcription factor with zinc finger DNA-binding } \\
\text { motif }\end{array}$ & 0.60 \\
\hline LEU3 & orf19.11700 & Predicted zinc-finger protein & 0.38 \\
\hline PPR1 & orf19.3986 & $\begin{array}{l}\text { Putative transcription factor with zinc cluster DNA-binding } \\
\text { motif }\end{array}$ & 0.43 \\
\hline RIM101 & orf19.7247 & Transcription factor involved in alkaline $\mathrm{pH}$ response & 0.36 \\
\hline
\end{tabular}




\begin{tabular}{lcll}
\hline ZCF2 & orf19.431 & Putative transcription factor with zinc cluster DNA-binding & 0.55 \\
ZCF20 & orf19.11621 & Predif & \\
ZCF32 & orf19.5940 & Predicted zinc-finger protein of unknown function & 0.40 \\
\hline
\end{tabular}

We determined whether up-regulation of these genes may affect the growth of double and $\sin 3 \Delta$ simple mutants on hydrogen peroxide. In the exponential phase of growth, $10^{7} \mathrm{cells} / \mathrm{ml}$ of each strain were spotted on YEPD medium supplemented with different concentration of $\mathrm{H}_{2} \mathrm{O}_{2}$. We observed that the double mutant like $\sin 3 \Delta \operatorname{simple}$ mutant showed more resistance to hydrogen peroxide $\left(\mathrm{H}_{2} \mathrm{O}_{2}\right)$ (Fig. 3.11) suggesting that SIN3 plays a role in regulation this set of genes.

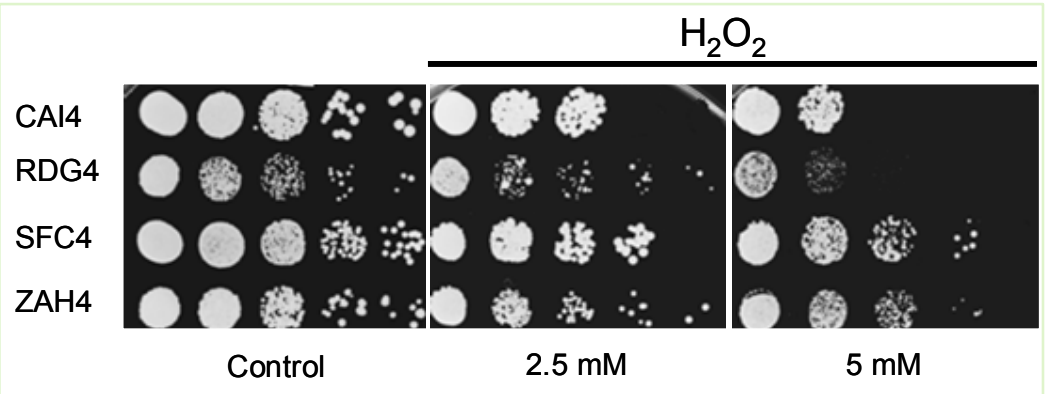

Figure 3.11 Sensitivity of the ZAH4 $(g c n 5 \Delta, \sin 3 \Delta)$ to the hydrogen peroxide $\left(\mathrm{H}_{2} \mathrm{O}_{2}\right)$

The transcription level four genes involved in the oxidation of fatty acids and peroxisomal activity including ECI1 (Lan et al., 2002; Uhl et al., 2003), POT1 (Enjalbert \& Whiteway, 2005), POX1-3 and SPS20 were increased. It has been observed that the $\beta$-oxidation of fatty acids is associated with the activity of peroxisomes (e.g. with the onset of the response to oxidative stress) (Koerkamp et al., 2002; Lan et al., 2002) and up-regulation of numerous genes involved in fatty acid oxidation (Pile et al., 2003) has been reported in Drosophila in agreement with our results.

\section{Genes coding for transcription factors}

Sixteen genes encode transcriptional regulators were found to be regulated by double mutant (Table 3.3). Four of them were up-regulated and twelve were down-regulated. Two of the four up-regulated, ZCF1 (Park et al., 2003) and ZPR1 (Rogers \& Barker, 2003), and six of twelve down-regulated, IPF1731, IPF29, PPR1, ZCF2, ZCF20, ZCF32 transcriptional regulators are encoded by genes that previously characterized as transcription factors with a zinc cluster DNA-binding motif. This family of transcriptional regulators may be a major target for both proteins in C. albicans and their role deserves further analysis. Other transcriptional factors (Table 3.3) belong to disperse families of regulators that respond to different factors and regulate a variety of 
genes with different functions. None of them are regulated in the gcn5 $\Delta$ and $\sin 3 \Delta$ simple mutants except of RIM101, PPR1 and LEU3 which have been reported to be up-regulated in the gcn5 $\Delta$ simple mutant.

\section{Other transcripts}

We have detected 4 genes, including NUM11, NUM12, TUB1 and TUB2 up-regulated in the double mutant, two of them -NUM12 and TUB1- have been reported to be upregulated in the $\sin 3 \Delta$ simple mutant. $S$. cerevisiae num1 and tub1 mutants showed a defect in correct nuclear migration (Heil-Chapdelaine et al., 2000; Schatz et al., 1988). Over-expression of alpha- and beta-tubulin genes in $S$. cerevisiae, separately or together, leads to accumulation of large excesses of each of the polypeptides, arrest cell division and forms abnormal structures. Excess beta-tubulin also might prevent microtubule elongation and over-expression of ScTUB1 temporally blocked normal cell cycle during the logarithmic growth phase (Weinstein \& Solomon, 1990). This result also could be explaining large cell morphology of the double and $\sin 3 \Delta$ simple mutant. Transcript levels of seven genes coding for cell wall proteins including PGA4, PGA14, PGA23, PGA54, PGA56 (Castillo et al., 2008), PMI1 (Yin et al., 2004), PMT2 (Prill et al., 2005) and three genes involved in the chitin anabolism: CHS3 (Mio et al., 1996), CHT2 (McCreath et al., 1995) and CHT3 (Dunkler et al., 2005; McCreath et al., 1995; McCreath et al., 1996) were up-regulated. None of them has been reported to be regulated in the both simple mutants, suggested that both genes regulate in a join way, an important set of cell wall genes.

Four of the seven genes coding for regulatory enzymes of the citric acid cycle, including succinate-CoA ligase, LSC2, (Kadosh \& Johnson, 2005; Lan et al., 2004; Murad et al., 2001a), malate dehydrogenase, MDH1, (Maglott et al., 2007), isocitrate dehydrogenase, IDP2, (Lan et al., 2002) and fumarate hydratase, FUM12 and four genes involved in glycolysis including ALD5 (Brand et al., 2004), FBA1 (Doedt et al., 2004; Garcia-Sanchez et al., 2004; Nantel et al., 2002), FBP1 (Doedt et al., 2004) and PCK1 (Leuker et al., 1997) were up-regulated in the double mutant. Some of genes involved in glycolysis pathway including ALD5, GPH5, GLK4, PDC11 and PYC2 has been reported to be up-regulated in the gcn5 $\Delta$ simple mutant (Degano, Ph.D thesis in process). None of them except IPD2 has been regulated in the $\sin 3 \Delta$ mutant under yeast mode of growth. Our results remarkably are in agreement with those reported in the Drosophila sin3 $\Delta$ mutant (Pile et al., 2003). 
Next, we focused on the identification of genes whose expression was altered in the double mutant strain during the yeast-hypha transition. We generated the transcriptional profiles for the gcn5 $\sin 3 \Delta$ double mutant in Lee medium for 15,60 and $180 \mathrm{~min}$ at $37^{\circ} \mathrm{C}$ using the CAl4 strain as reference. A total of 1271 genes were found to change at the consensus level of $\geq 1.5$ fold, including 690 up- and 581 downregulated genes. The distribution of genes altered at the three points is represented in the Venn diagram, and the clustering of genes regulated by deletion of GCN5 and SIN3 is represented in Fig. 3.12 ( $A$ and $B$ ). We found that 43 genes were common to the three times assayed (10 and 33 up- and down- respectively), 156 genes at 15 and 60 min (102 up- and 54 down-) and 47 genes at 60 and 180 min (9 up- and 38 down-). 324 genes (227 up- and 97 down-), 645 genes (315 up- and 330 down-) and 51 genes (24 up- and 27 down-) varied only at one time of induction (at 15, 60 and $180 \mathrm{~min}$ respectively). However, only 5 genes were common between 15 and 180 min validating our experimental approach. The elevated number of regulated genes detected only at one time suggests that transitory changes occur sequentially in response to both mutations during the morphogenetic switch (Fig. 3.12).

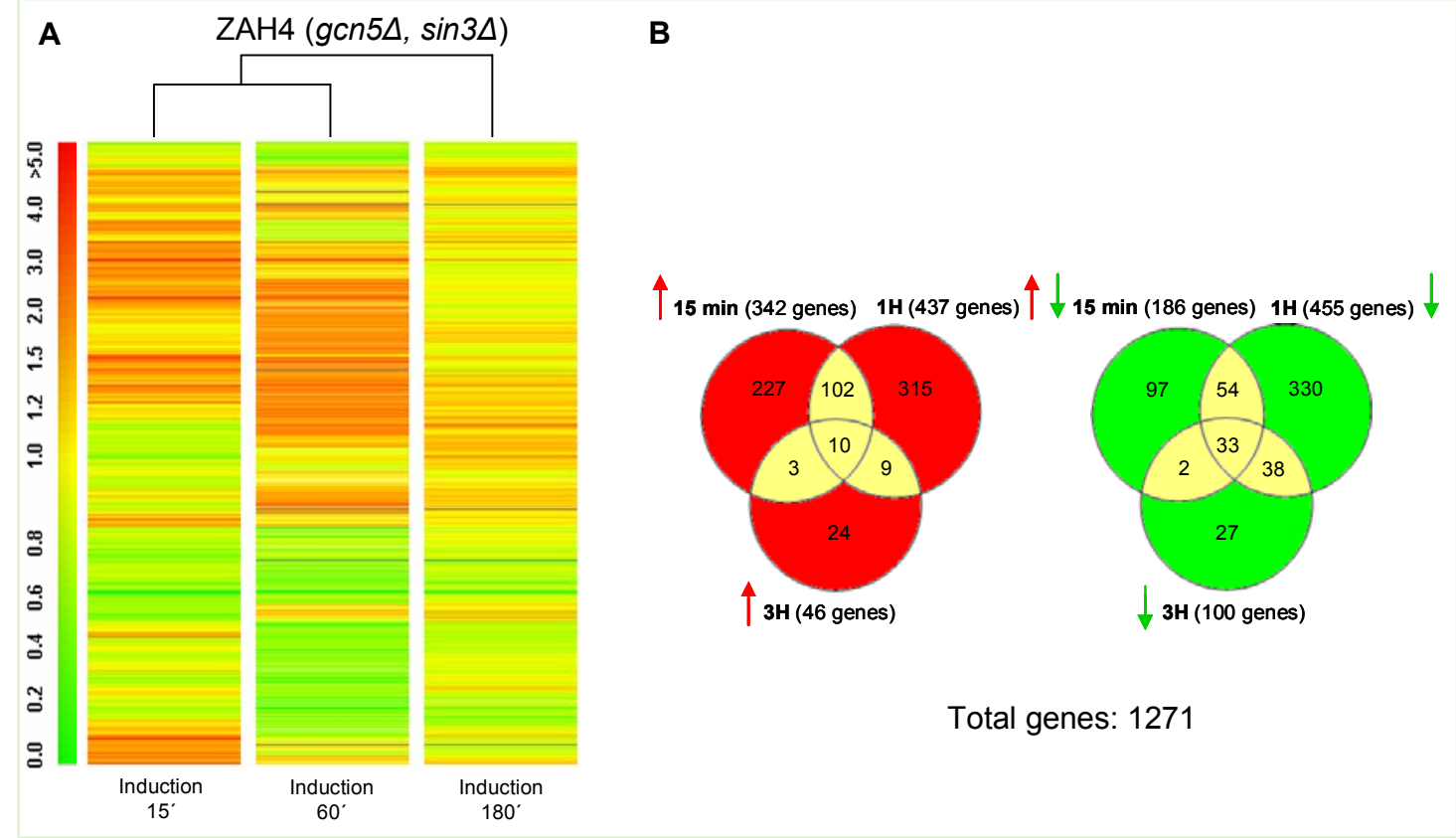

Figure 3.12 Transcript profiles of $C$. albicans $g c n 5 \Delta, \sin 3 \Delta$ double mutant during the yeast-hypha transition (A). Venn diagrams of genes regulated by Gcn5p and $\operatorname{Sin} 3 p$ in the double mutant (B). The CAI4 strain was used as reference, which all were grown in identical conditions: Lee $/ 37^{\circ} \mathrm{C}$ (inducing). 
Forty three genes common at the three times are listed in Table 3.4. Only 2 of the common genes correspond to genes of unknown function. Of the rest of the genes, 16 belong to disperse functional categories i.e., HSP12 heat-shock protein, ACC1 protein with similarity to acetyl-coenzyme-A carboxylases, etc., and 25 genes can be grouped in seven functional categories. The main category corresponds to cell wall proteins and includes the down-regulation of HWP1 a well known cell wall protein (Staab et al., 1999), ECE1 a protein involved in cell elongation (Bennett et al., 2003; Birse et al., 1993) and RBT1 (Bennett et al., 2003; Sohn et al., 2003) a putative cell wall protein with similarity to Hwp1p, two genes belonging to the ALS family, including ALS1 and ALS3. All these down-regulated genes have been described previously as being upregulated during the hypha transition (Braun et al., 2005; Nantel et al., 2002) and some are regulated by Efg1p and Tup1p (Doedt et al., 2004; Kadosh \& Johnson, 2005; Sohn et al., 2003). Another class of well-known genes regulated at three times consists of genes belonging to the following categories: fatty acid metabolism ( 5 genes), electron transporters (3 genes), oxidases/peroxidses (4 genes), vitamin biosynthesis ( 2 genes) and carbohydrate metabolism (3 genes).

Table 3.4 Commom differentially expressed genes in $g c n 5 \Delta, \sin 3 \Delta$ mutant at the three times of incubation (at 15,60 and $180 \mathrm{~min}$ )

\begin{tabular}{|c|c|c|c|c|c|}
\hline $\begin{array}{l}\text { Gene } \\
\text { name }\end{array}$ & ORF & Description & $15 \mathrm{~min}$ & $60 \mathrm{~min}$ & $180 \mathrm{~min}$ \\
\hline \multicolumn{6}{|c|}{$\begin{array}{l}\text { Down-regulated genes } \\
\text { Cell wall }\end{array}$} \\
\hline ALS1 & orf19.5741 & Adhesin; ALS family of cell-surface glycoproteins & 0.10 & 0.11 & 0.20 \\
\hline ALS3 & orf19.2355 & Agglutinin like protein & 0.21 & 0.33 & 0.09 \\
\hline ECE1 & orf19.3374 & Protein comprising eight 34-residue repeats & 0.03 & 0.06 & 0.05 \\
\hline HWP1 & orf19.1321 & Hyphal cell wall protein & 0.08 & 0.23 & 0.29 \\
\hline MP65 & orf19.9345 & Cell surface mannoprotein & 0.33 & 0.29 & 0.50 \\
\hline PGA58 & orf19.4334 & $\begin{array}{l}\text { Putative GPI-anchored protein of unknown } \\
\text { function }\end{array}$ & 0.59 & 0.50 & 0.53 \\
\hline RBT1 & orf19.1327 & Putative cell wall protein with similarity to Hwp1p & 0.40 & 0.29 & 0.52 \\
\hline RHR2 & orf19.5437 & Putative glycerol 3-phosphatase & 0.25 & 0.24 & 0.48 \\
\hline \multicolumn{6}{|c|}{ Fatty acid metabolism } \\
\hline ERG251 & orf19.12101 & Ketoconazole-induced & 0.44 & 0.11 & 0.48 \\
\hline FAD2 & orf19.7765 & Delta-12 fatty acid desaturase & 0.53 & 0.46 & 0.60 \\
\hline FAS2 & orf19.5948.1 & $\begin{array}{l}\text { Fatty-acyl-CoA synthase, alpha chain, internal } \\
\text { fragment }\end{array}$ & 0.22 & 0.43 & 0.51 \\
\hline HET3 & orf19.6327 & Putative sphingomyelin transfer protein & 0.41 & 0.39 & 0.57 \\
\hline OLE1 & orf19.5117 & Fatty acid desaturase (stearoyl-CoA desaturase) & 0.31 & 0.39 & 0.57 \\
\hline \multicolumn{6}{|c|}{ Oxidase/peroxidase } \\
\hline FET3 & orf19.11687 & Multicopper oxidase & 0.62 & 0.24 & 0.52 \\
\hline FET34 & orf19.4215 & Protein similar to multicopper ferroxidase & 0.48 & 0.49 & 0.52 \\
\hline GPX2 & orf19.85 & Similar to glutathione peroxidase & 0.15 & 0.25 & 0.51 \\
\hline SOD5 & orf19.2060 & $\begin{array}{l}\text { Copper- and zinc-containing superoxide } \\
\text { dismutase }\end{array}$ & 0.52 & 0.14 & 0.32 \\
\hline \multicolumn{6}{|c|}{ Transcription factors } \\
\hline GAT2 & orf19.4056 & Putative DNA-binding transcription factor & 0.54 & 0.37 & 0.46 \\
\hline UME6 & orf19.9381 & $\begin{array}{l}\text { Transcription factor; required for wild-type hyphal } \\
\text { extension }\end{array}$ & 0.37 & 0.25 & 0.62 \\
\hline \multicolumn{6}{|c|}{ Charbohydrate metabolism } \\
\hline $\mathrm{ADH} 1$ & orf19.3997 & $\begin{array}{l}\text { Alcohol dehydrogenase; at surface of yeast-form } \\
\text { cells but not hyphae }\end{array}$ & 0.32 & 0.08 & 0.24 \\
\hline HGT7 & orf19.2023 & $\begin{array}{l}\text { Putative glucose transporter, major facilitator } \\
\text { superfamily }\end{array}$ & 0.40 & 0.07 & 0.23 \\
\hline
\end{tabular}




\begin{tabular}{|c|c|c|c|c|c|}
\hline PFK2 & orf19.6540 & Beta subunit of phosphofructokinase (PFK) & 0.35 & 0.12 & 0.49 \\
\hline \multicolumn{6}{|c|}{ Vitamin biosynthesis } \\
\hline FCY24 & orf19.7331 & Putative transporter & 0.45 & 0.28 & 0.54 \\
\hline THI13 & orf19.7324 & $\begin{array}{l}\text { Protein not essential for viability; similar to } S \text {. } \\
\text { cerevisiae Thi } 13 p\end{array}$ & 0.50 & 0.16 & 0.50 \\
\hline \multicolumn{6}{|c|}{ Other function } \\
\hline ACC1 & orf19.7466 & $\begin{array}{l}\text { Protein with similarity to acetyl-coenzyme-A } \\
\text { carboxylases }\end{array}$ & 0.30 & 0.40 & 0.47 \\
\hline ARP3 & orf19.2289 & $\begin{array}{l}\text { Shows Myo5p-dependent localization to cortical } \\
\text { actin patches at hyphal tip }\end{array}$ & 0.18 & 0.16 & 0.41 \\
\hline CAN5 & orf19.3641 & $\begin{array}{l}\text { Expression is regulated upon white-opaque } \\
\text { switching }\end{array}$ & 0.57 & 0.26 & 0.50 \\
\hline DEF1 & orf19.7561 & $\begin{array}{l}\text { Protein required for filamentous growth and for } \\
\text { escape from epithelial cells }\end{array}$ & 0.38 & 0.24 & 0.48 \\
\hline DUR3 & orf19.6656 & Putative protein of unknown function & 0.38 & 0.27 & 0.54 \\
\hline IPF1548 & orf19.951 & $\begin{array}{l}\text { Transcription downregulated upon yeast-hyphal } \\
\text { switch }\end{array}$ & 0.44 & 0.43 & 0.49 \\
\hline IPF8762 & orf19.822 & Protein detected in some, not all, biofilm extracts & 0.49 & 0.16 & 0.44 \\
\hline SAM4 & orf19.386 & Alkaline upregulated & 0.54 & 0.39 & 0.43 \\
\hline YHB1 & orf19.3707 & Nitric oxide dioxygenase & 0.40 & 0.27 & 0.26 \\
\hline \multicolumn{6}{|c|}{$\begin{array}{l}\text { Up-regulated genes } \\
\text { Electron transport }\end{array}$} \\
\hline ATP6 & & $\begin{array}{l}\text { Subunit } 6 \text { of the F0 sector of mitochondrial F1F0 } \\
\text { ATP synthase }\end{array}$ & 3.73 & 2.53 & 1.76 \\
\hline coX1 & & Subunit I of cytochrome c oxidase & 2.93 & 1.78 & 1.86 \\
\hline COX3A & & Subunit III of cytochrome c oxidase & 2.51 & 2.07 & 1.87 \\
\hline \multicolumn{6}{|c|}{ Other function } \\
\hline ALD5 & orf19.13228 & $\begin{array}{l}\text { Protein described as an aldehyde } \\
\text { dehydrogenase }\end{array}$ & 3.12 & 1.77 & 2.12 \\
\hline HSP12 & orf19.3160 & $\begin{array}{l}\text { Heat-shock protein; induced } \\
\text { osmotic/oxidative/cadmium stress }\end{array}$ & 24.53 & 7.23 & 2.23 \\
\hline IPF15217 & orf19.3779 & Unknown function & 2.94 & 7.83 & 1.85 \\
\hline IPF4065 & orf19.1862 & Possible stress protein & 10.87 & 4.16 & 2.46 \\
\hline IPF4510 & orf19.6999 & Unknown function & 1.53 & 2.30 & 1.83 \\
\hline IPF6881 & orf19.4246 & Putative phosphatidyl synthase & 7.32 & 3.01 & 2.14 \\
\hline PUT2 & orf19.3974 & Alkaline upregulated & 4.47 & 4.32 & 2.24 \\
\hline
\end{tabular}

\section{Expression of transcription factors}

Only two transcription factor was down-regulated at the three times of incubation; namely, GAT2, which has been described as a putative DNA-binding transcription factor with unspecified role in morphogenesis, that await further characterization and UME6 is a transcription factor; required for wild-type hyphal extension (Banerjee et al., 2008). Deletion of UME6 gene produces clear defects in hyphal formation and extension, and hence our results are in agreement with phenotype of double mutant during the hypha transition. Two transcriptional factors -ARG83 and ECM22- were down-regulated at two times: 15 and 60 min. Six genes including BDF1 (Bensen et al., 2002; Marchais et al., 2005), HIR2 (DeSilva et al., 1998; Spector et al., 1997), IPF1731 a putative transcription factor transcriptionally regulated upon hyphal formation (Murillo et al., 2005; Nobile \& Mitchell, 2005; Park et al., 2003), NRG1 (Murad et al., 2001b), NUT2 (Gustafsson et al., 1998) and SWI4 (Castillo et al., 2006) were up- and two genes including LEU3 (Bensen et al., 2004; Maicas et al., 2005; Ramsdale et al., 2008), ZCF32 (Maicas et al., 2005; Znaidi et al., 2007) and ZFU2 a putative transcription factor with zinc finger DNA-binding motif (Mitrovich et al., 2007; Nobile \& 
Mitchell, 2005) were down-regulated at $15 \mathrm{~min}$. Some of transcription factors are regulated in the yeast-hypha transition, as described from previous studies (Banerjee et al., 2008; Murad et al., 2001 a; Murillo et al., 2005; Nobile \& Mitchell, 2005; Park et al., 2003; Rashki, 2009) and differentially expression of these genes in the both simple mutants and CAI4 strain are shown in Fig 3.13. Together these results, which fit nicely with previous data regarding hypha-transition, strongly support the validity of our microarray analysis.

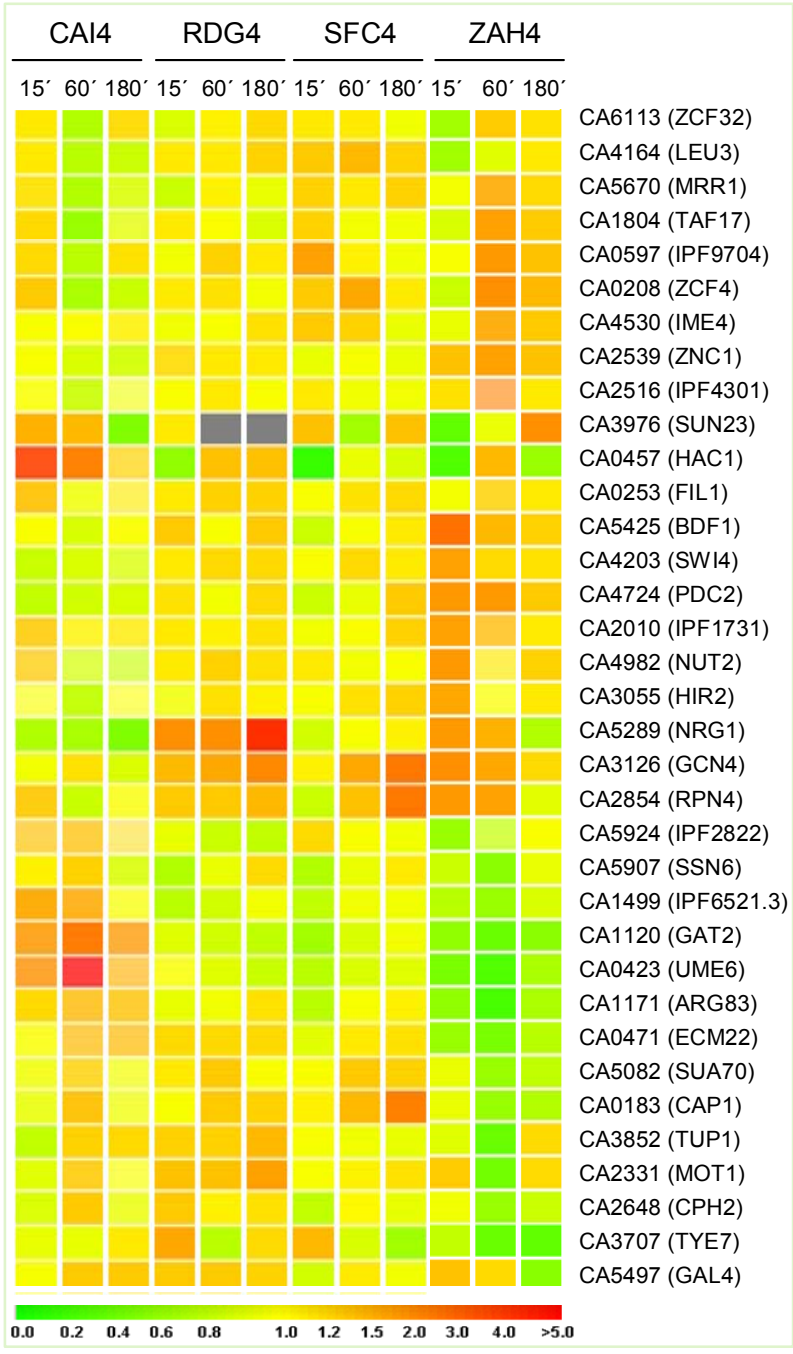

Figure 3.13 Cluster analysis of genes encoding for transcriptional factors in the CAI4, RDG4 (gcn5 $\Delta$ ), SFC4 $(\sin 3 \Delta)$ and ZAH4 $(g c n 5 \Delta, \sin 3 \Delta)$ strains during the yeast-hypha transition.

\section{Time course of gene induction of the main significant categories}

The genes belonging to diverse functional categories whose expression level changed at least at one time of incubation were clustered together. These diverse functional categories include genes that are both up- and down-regulated, indicating a differential regulation of these functions by the double mutant. Many genes were could not be 
readily categorized into functional groups and represent a wide range of cellular metabolic processes (Supplementary tables 3.3 and 3.4). The microarray results demonstrate that the lack of Gcn5p and $\operatorname{Sin} 3 p$ affect a large number of cell functions and most likely serve to globally reprogram of $C$. albicans physiology that allow the double mutant to adapt to this environment.

Cell wall genes: Genes encode cell wall proteins were also altered by loss of Gcn5p and $\operatorname{Sin} 3 p$ during hypha transition. For example, the ALS family includes 8 genes that five of them were down-regulated included $A L S 1$ and $A L S 3$ at the tree times, $A L S 2$ (Hoyer et al., 1998b; Zhao et al., 2005b), ALS9 (Zhao et al., 2003) at 15 min and ALS4 (De Groot et al., 2003) at 15 and $60 \mathrm{~min}$ (Fig. 3.14A). ALS1 and ALS3 has been reported to be up-regulated during the hypha transition in the CAI4 strain at $28-37^{\circ} \mathrm{C}$ temperature shifts in the Lee medium (Rashki, Ph.D thesis 2009).

HYR1 a predicted cell wall protein which described as glutathione peroxidase and regulated by Rfg1p, Efg1p, Nrg1p, Tup1p, Cyr1p (Fernandez-Arenas et al., 2007; Kadosh \& Johnson, 2001; Murad et al., 2001b) is down-regulated at 15 and $60 \mathrm{~min}$. Two genes, CHS3 (Mio et al., 1996; Munro et al., 1998), at $60 \mathrm{~min}$, and YWP1, at 180 min, were up-regulated. Down-regulation of YWP1 has been reported in the CAI4 strain during hypha transition (Rashki, Ph.D thesis 2009). This finding is consistent with the phenotypic results, which indicated that the double mutant failed to form true hypha in either liquid or solid media.

GPI anchored proteins: Our results indicate that the transcript levels of seventeen genes belonging to a large class of functionally diverse proteins (GPI-anchored proteins) were regulated in the double mutant at least at one time during the hypha transition. Of these, PGA1 (Castillo et al., 2006; De Groot et al., 2003), PGA49 (De Groot et al., 2003), CWH41 (Mora-Montes et al., 2007), ECM1 (Harcus et al., 2004), FGR12 (Uhl et al., 2003), FGR41 (De Groot et al., 2003), IFF5 (De Groot et al., 2003) were up-regulated and KRE1 (found highly regulated during $C$. albicans protoplast regeneration) (Castillo et al., 2006), PGA6, PGA45, PGA53, PGA62 and PGA63, ECM21, ECM33, FGR6, PHR1 were down-regulated at $60 \mathrm{~min}$ (Fig. 3.14B). The transcript levels of PGA37 (De Groot et al., 2003) at 15 min and PGA54 a putative GPIanchored protein (De Groot et al., 2003), RBT4 which required for virulence in mouse systemic (Garcia-Sanchez et al., 2005; Sohn et al., 2003) at 15 and 60 min were downregulated (Fig. 3.14B).

Regulation a large number of genes coding for putative GPI-anchored proteins in the C. albicans CAl4 (22 genes), gcn5 $\Delta$ (10 genes), hda1 $\Delta$ (5 genes) and gcn5 $\Delta$, hda1 $\Delta$ 
double mutant (16 genes) has been reported in our laboratory. From our results and those described by other, it may be concluded that the morphogenetic switch involves a strong remodelling of the yeast cell wall and membrane.

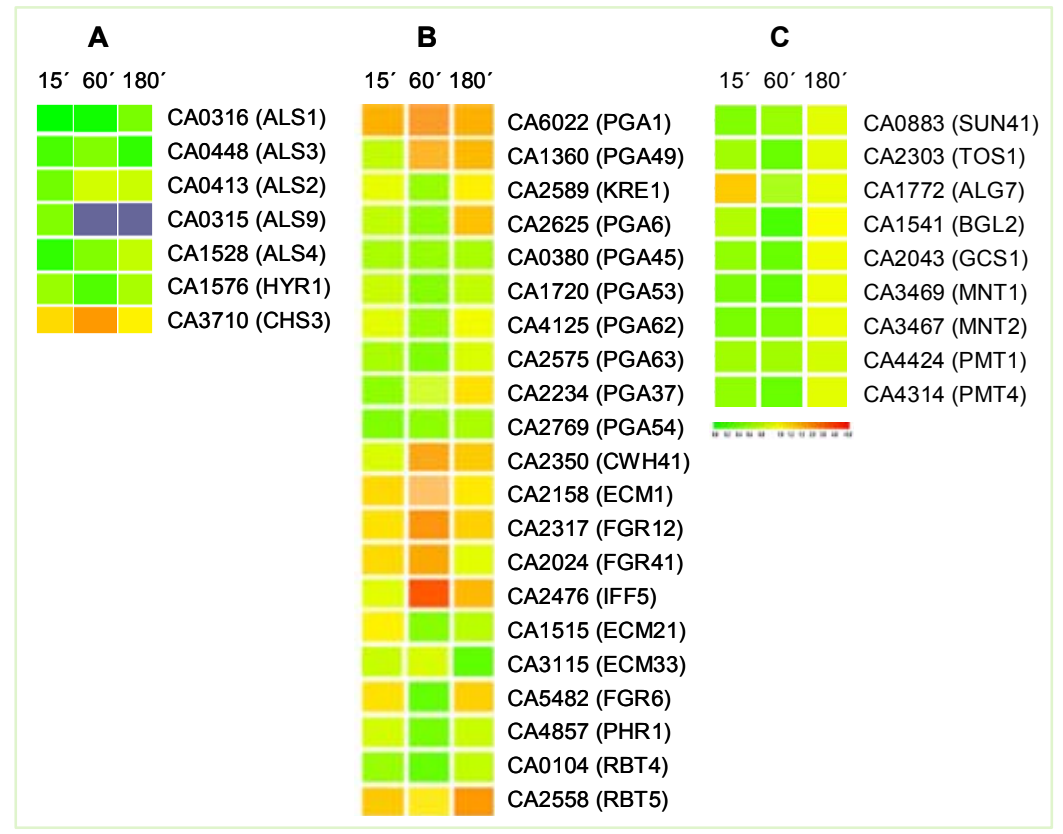

Figure 3.14 Transcriptional response of genes involved in the cell wall in the $\Delta g c n 5, \Delta \sin 3$ double mutant. Representative genes responsible for specific cellular functions are grouped and their regulation during 15,60 and 180 min of yeast-hypha transition in the $\Delta g c n 5, \Delta \sin 3$ double mutant is shown in the color bar diagrams.

Glucanases, glucosidases and mannosyltransferases: Our microarray analysis indicated that seven genes encoding glucanases, glucosidases and mannosyltransferases including SUN41 a putative cell wall glycosidase which is involved in biofilm formation and required for cell separation (Firon et al., 2007) and TOS1 a protein described as similar to alpha agglutinin anchor subunit (Bader et al., 2008), GCS1 subunit of beta-1,3-glucan synthase (Braun et al., 2005; Mio et al., 1997), MNT1 a Alpha-1,2-mannosyl transferase (Braun et al., 2005; Buurman et al., 1998), MNT2 a Alpha-1,2-mannosyl transferase (Braun et al., 2005; Munro et al., 2005), PMT1 a mannosyltransferase (Prill et al., 2005; Timpel et al., 1998) and PMT4 a mannosyltransferase required for normal cell wall composition and full virulence in mouse systemic infection (Prill et al., 2005) were down-regulated at 15 and $60 \mathrm{~min}$. Deletion of both alleles of the SUN41 resulted in defects of hyphal formation (Firon et al., 2007) that is in agreement with phenotype of double mutant during the hypha transition (Fig. 3.14C).

Genes involved in protein synthesis and ribosomal proteins: A second important category includes up-regulation of NOC4, NOP2, NOP10, RPB8, RPC10, RPC31, 
RPC82, RPL25, RPL26A, RPL27A, RPL35, RRP1, RRP3, RRP9, RRS1, RSA2, SIK1 and down-regulation of RPL2, RPL7A, RPL13, RPL14, RPL15A, RPL18, RPL30, RPN2, RPP2A, RPP2B, RPS4A, RPS16A, RPS17B, RPS20 genes at 60 min of hypha transition. No genes of this category were found up- and down-regulated at other time point. Differentially expressions of some of them are reported in the gcn5 $\Delta$ and $\sin 3 \Delta$ simple mutants during hypha transition. Almost no data about the behavior of this group of genes under standard conditions of the yeast-hypha transition (serum or temperature) have been reported. Three of them (RPL27A, RPS4A and RPS20) have been described as up-regulated under standard conditions of the yeast-hypha transition (temperature) in CAl4 strain (Rashki, Ph.D thesis 2009). Five of them (RPL13, RPL25, RPL26A, RPL30 and RPS4A) have been reported up-regulated by comparison of Cacwt1 mutant with a wild type strain (Reuss \& Morschhauser, 2006) and finally ten of them were down-regulated in the $C$. albicans gcn5 $\Delta$ mutant during yeast form growth (Degano, Ph.D thesis in process). Differencially expression of this set of genes in the CAI4 and both simple mutants is shown in Fig. 3.15.

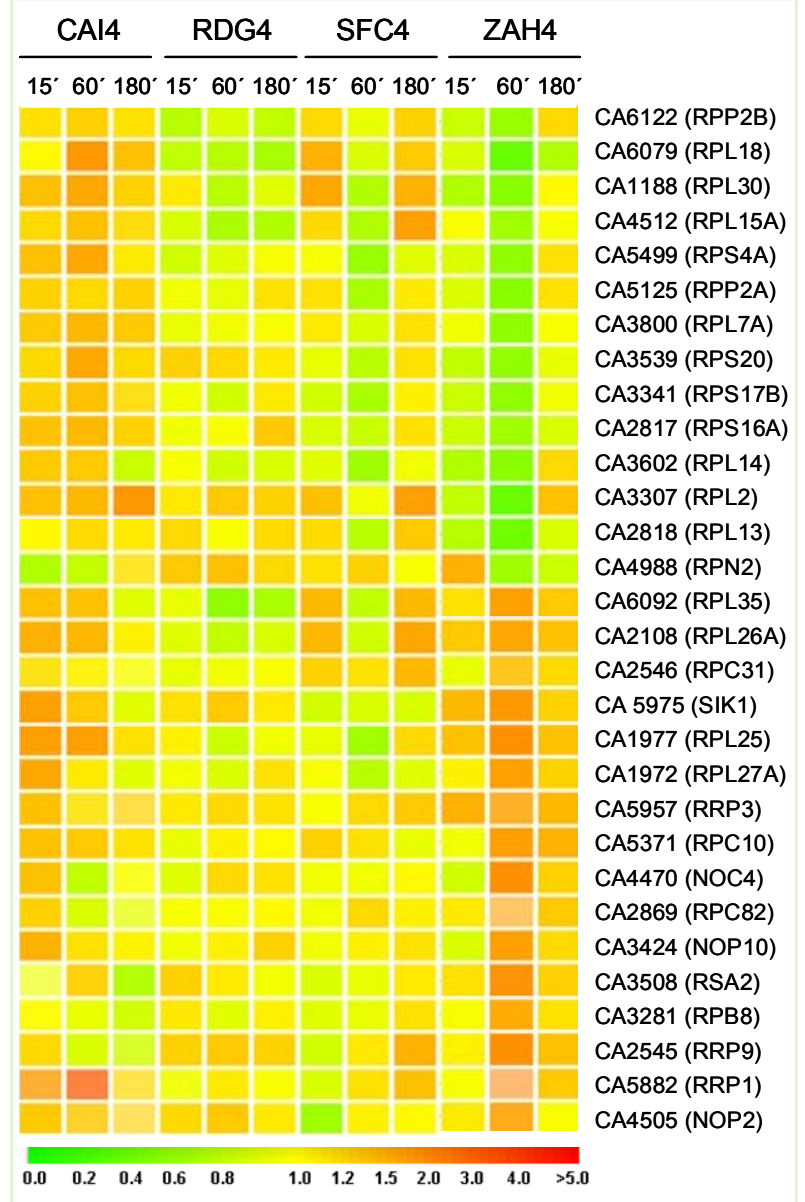

Figure 3.15 Cluster analysis of genes encoding for ribosomal proteins in the CAI4, RDG4 (gcn5 $\Delta$ ), SFC4 $(\sin 3 \Delta), \mathrm{ZAH} 4(g \operatorname{cn} 5 \Delta, \sin 3 \Delta)$ strains during the yeast-hypha transition. 
Central carbon metabolism: The regulation of 34 genes (27 down-regulated after 60 min of incubation) involved in the glycolytic pathway, the TCA and glyoxylate cycles and gluconeogenesis (see Fig. 3.16A and B; Fig. 3.17) were detected. Expression some of them has been reported to be decreased in the $g c n 5 \Delta(180 \mathrm{~min})$ and $\sin 3 \Delta$ (60 and $180 \mathrm{~min}$ ) (Fig. 3.17). In general our results are in agreement with those described in the $g c n 5 \Delta$, hda1 $\Delta$ double mutant during the yeast-hypha transition (Rashki, Ph.D thesis 2009). These changes reflect differences in the modulation of metabolic genes during the hypha transition.

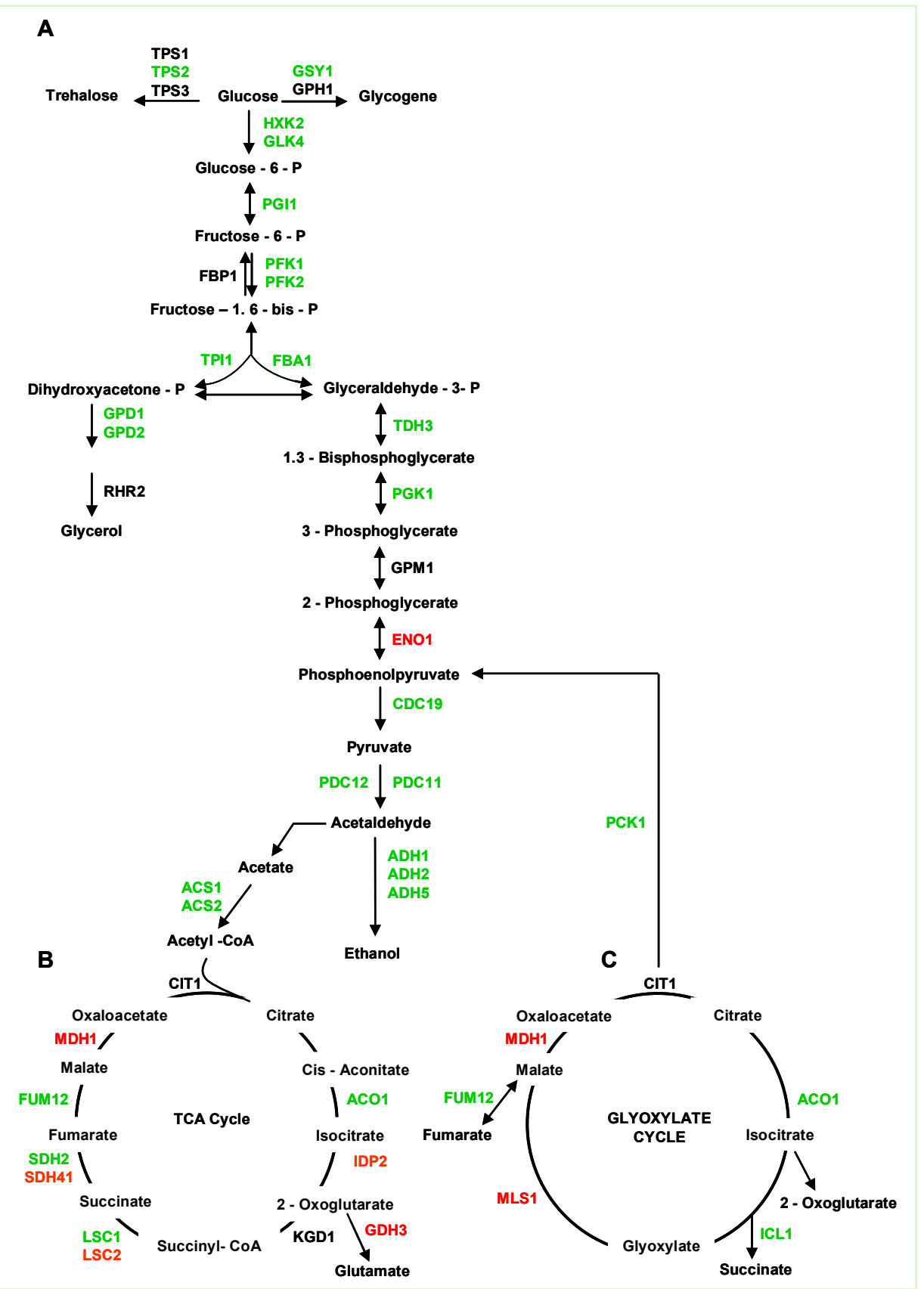

Figure 3.16 Transcriptional regulation of genes involved in the glycolitic pathway (A), TCA cycle (B) and glyoxylate cycle $(\mathrm{C})$ in the $g \operatorname{cn} 5 \Delta, \sin 3 \Delta$ double mutant. 


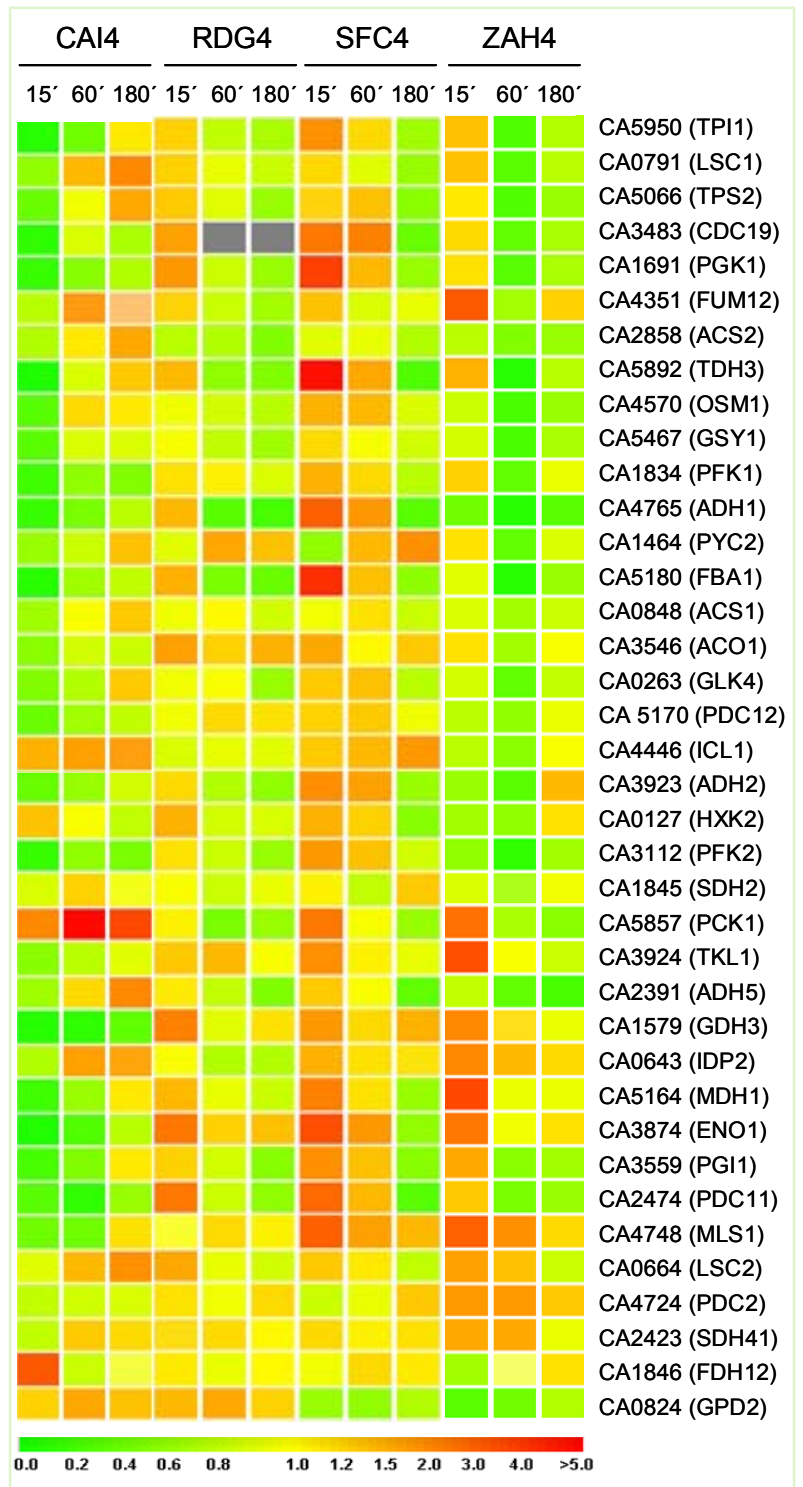

Figure 3.17 Cluster analysis of genes involved in carbohydrate metsabolism in the CAI4, RDG4 $(g c n 5 \Delta)$, SFC4 $(\sin 3 \Delta)$ and ZAH4 $(g \operatorname{con} 5 \Delta, \sin 3 \Delta)$ mutants during the yeast-hypha transition.

However, their relationship to morphogenesis has not been clarified; even it has been suggested to be due to physiological changes underlying the shift in growth conditions and not to the morphogenetic process itself (Swoboda et al., 1994). We have found down-regulation of five genes belonging to the glucose transporter HGT1 (Walker et al., 2009), HGT2 (Fan et al., 2002), HGT6 (Fan et al., 2002), HGT7 and HGT8 (Fan et al., 2002) at $60 \mathrm{~min}$ (Fig. 3.18). 


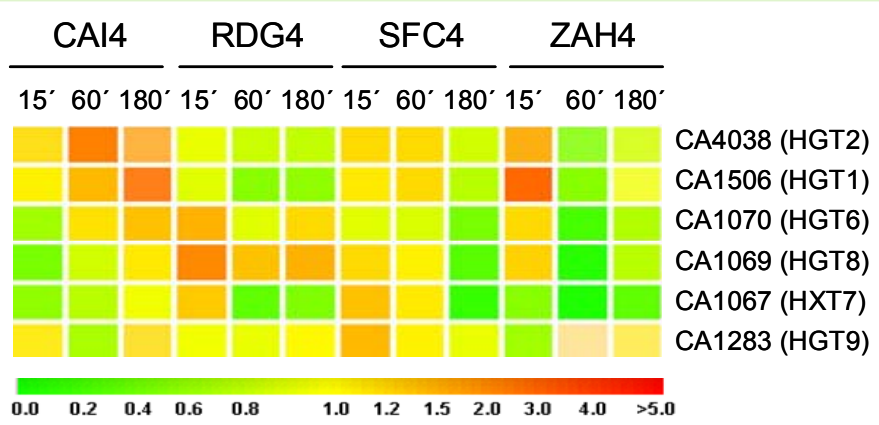

Figure 3.18 Cluster analysis of genes inolved in glucose transporter in the CAI4, RDG4 (gcn5 $\Delta$ ), SFC4 $(\sin 3 \Delta), \mathrm{ZAH} 4(g c n 5 \Delta, \sin 3 \Delta)$ strains during the yeast-hypha transition.

Cell polarity and signal transduction: A number of genes involved in cell polarity and signal transduction e.g. AIP2, RAC1, RAS1, RGS2, RHO3 and RSR1 were downregulated at $60 \mathrm{~min}$ (Supplementary table 3.4). Among these, CDC42 (Bassilana et al., 2005; Mirbod et al., 1997; Ushinsky et al., 2002), GPR1 (Maidan et al., 2005; Miwa et al., 2004; Prigneau et al., 2003), RAC1 (Bassilana \& Arkowitz, 2006) and RSR1 (Bassilana et al., 2003; Yaar et al., 1997) has been described to be required for wildtype hyphal growth.

The down-regulation of three components of the secratory pathway IPF1022, SEC24 possible role in ER to Golgi transport and induced upon yeast-hyphal switch (Nantel et al., 2002) and SEC61, similar to S. cerevisiae Sec61p act in protein translocation from the endoplasmic reticulum (de la Rosa et al., 2004; Ruiz et al., 2001) were detected at 15 and 60 min of incubation. Down-regulation this set of genes has been reported in the $g c n 5 \Delta$, hda1 $\Delta$ double mutant during one time of hypha transition. None of them were regulated in the $\sin 3 \Delta$ and gcn5 $\Delta$ simple mutants. Transcription level genes including GPA2, GPR1, CA2348, RAC1, RGS2, AIP2, RHO3, RAS1 and RSR1 have been reported that increased in the CAI4 strain and decrease in the gcn5 $\Delta$ and $\sin 3 \Delta$ simple mutants during hypha transition (Fig. 3.19). This result suggesting that Gcn5p and $\operatorname{Sin} 3 p$ have a role in the regulation of these genes. It appears that the physiological reprogramming by loss of $\mathrm{Gcn} 5 \mathrm{p}$ and $\operatorname{Sin} 3 p$ during hypha transition involves in pathways that decreases transcription of cell polarity and signal transduction and secretory pathway genes, although we do not presently know the functional reasons for this phenomenon. 


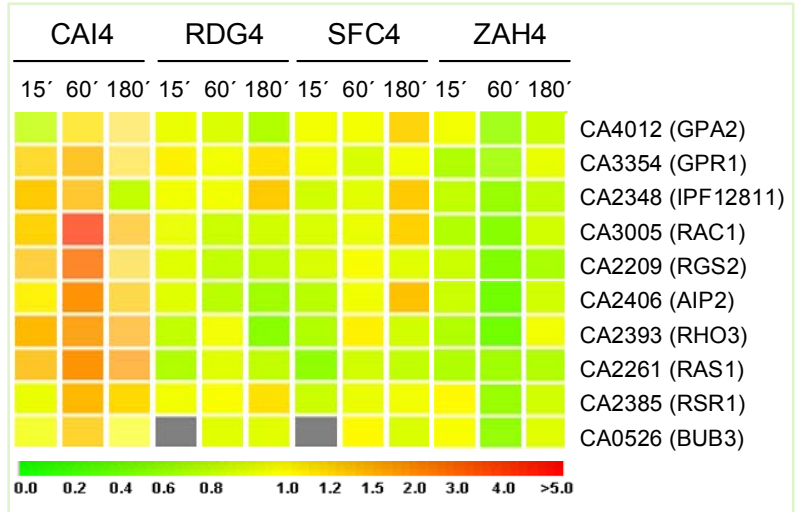

Figure 3.19 Cluster analysis of genes involved in signal transduction in the CAI4, RDG4 (gcn5 $\Delta$ ), SFC4 $(\sin 3 \Delta)$ and $\mathrm{ZAH} 4(g \operatorname{cn} 5 \Delta, \sin 3 \Delta)$ strains during the yeast-hypha transition

Vitamin biosynthesis: We have detected the down-regulation five genes involved in vitamin biosynthesis -BIO2 (Bruno \& Mitchell, 2005), BIO3 (Maglott et al., 2007), BIO4 (Maglott et al., 2007), THI4 (Braun et al., 2005; Zhao et al., 2005a) and THI20 (Braun et al., 2005)- at $60 \mathrm{~min}$, one of them that is fungal specific, THI4, identified in $S$. cerevisiae as the gene involved in the synthesis of HET-P, is a single gene but its transcript is one of the most abundant under thiamine-depleted conditions (Muller et al., 1999). The down-regulation of BIO2 at $180 \mathrm{~min}$ and two genes involved in thiamine biosynthesis (THI4 and THI13) at one and two times of incubation in the gcn5 $\Delta$ and $\sin 3 \Delta$ simple mutants has been described suggested role of Gcn5p and Sin $3 p$ in regulation of genes involved in vitamin biosynthesis. Expression of three genes involved in thiamine biosynthesis -THI4, THI6, and THI13- has been reported to be upregulated in the CAl4 hyphal growth (Rashki, Ph.D thesis 2009).

Set of genes regulated only at one incubation time: After 15 and 60 minutes of incubation at $37^{\circ} \mathrm{C}$, several sets of genes were either up- or down-regulated. The main groups of up-regulated genes (at $15 \mathrm{~min}$ ) included four genes contributing to oxidative stress response (GAD1, GRX3, SOD1 and TSA1) and CTA1 (Lan et al., 2004; Niewerth et al., 2003), MCR1 (Bensen et al., 2004), NAD1 (Karababa et al., 2004; Wang et al., 2006), SOD2 (Bensen et al., 2004; Enjalbert et al., 2006) and TRX1 at 15 and $60 \mathrm{~min}$. Other up-regulated genes at $15 \mathrm{~min}$ were four genes involved in biosynthesis of glutamate, aminoadipic acid pathway (LYS2, LYS9, LYS21 and LYS22) (Fig. 3.20), four genes of the $\mathrm{F}_{1} \mathrm{~F}_{0}$ ATPase complex (ATP1, ATP5, ENA2 and VMA2) and five genes of proteasome subunits (PR26, PRE1, PRE4, PRE5 and PRE6). The genes up-regulated at $60 \mathrm{~min}$ were five genes involved in peroxisomal assembly (PER3, PEX3, PEX10, PEX17 and SPS20), eight genes of mitochondrial (ALD4, ISA2, MDM12, MTF1, NAM2, NUC1, TIM9 and TIM10) (Fig. 3.20). 


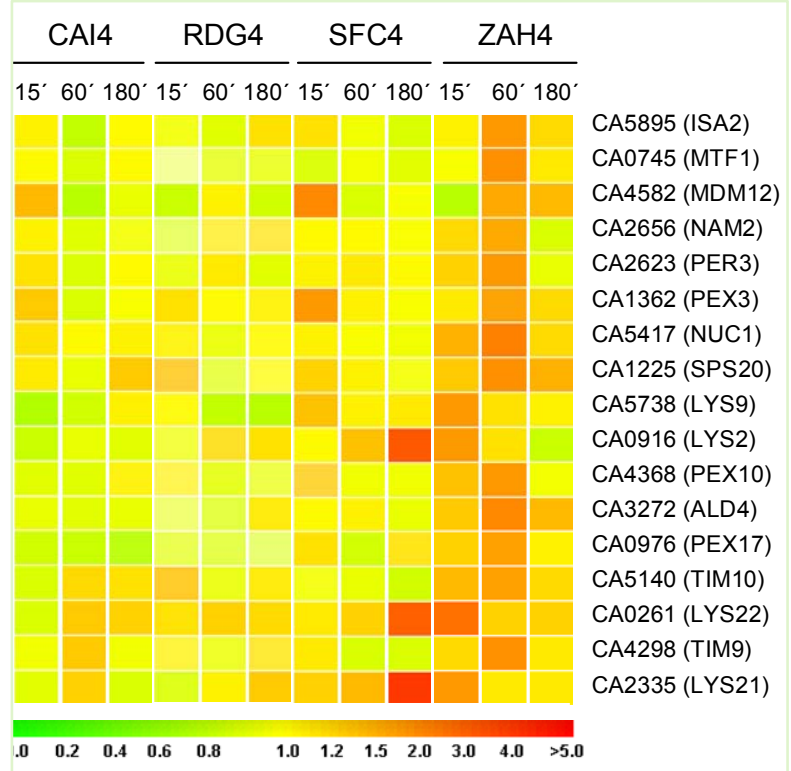

Figure 3.20 Cluster analysis of gene expression in the CAl4, RDG4 (gcn5 $\Delta$ ), SFC4 (sin $3 \Delta$ ) and ZAH4 $(g c n 5 \Delta, \sin 3 \Delta)$ strains during the yeast-hypha transition.

Among the down-regulated genes after $60 \mathrm{~min}$, we noted six genes corresponding to ergosterol biosynthesis (ERG1, ERG3, ERG4, ERG10, ERG11 and ERG13) (Fig. 3.21). The down-regulation most of them have been reported in the gcn5 $\Delta$, hda1 $\Delta$ double mutant and CAl4 strain during the yeast-hypha transition (Rashki, Ph.D thesis 2009) together with this result suggested a change in membrane composition during hypha formation.

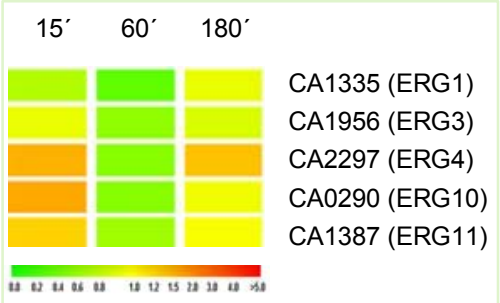

Figure 3.21 Differential expression of the genes involved in ergosterol biosynthesis

Clustering analysis these sets of genes in the double, both simple mutants and CAI4 strain during hypha transition (Fig. 3.22) was showed that transcription level genes including SOD1, SOD2, TRX1, PRE4, GAD1, CTA1 and MCR1 are decreased in the CAI4 (at 15 and $60 \mathrm{~min}$ ) and increased in both simple and double mutants indicate role of GCN5 and SIN3 in regulation genes involved in oxidative stress. As sowed in Fig.

transcription two genes involved in sodium transport, ENA21 and ENA22 are increased in the gcn5 simple, gcn5 $\Delta$, sin3 $\Delta$ double mutant and CAI4 strain and decreased in the $\sin 3 \Delta$ mutant during the hypha transition suggested role of $\operatorname{Sin} 3 p$ in the regulation of sodium transporter. 


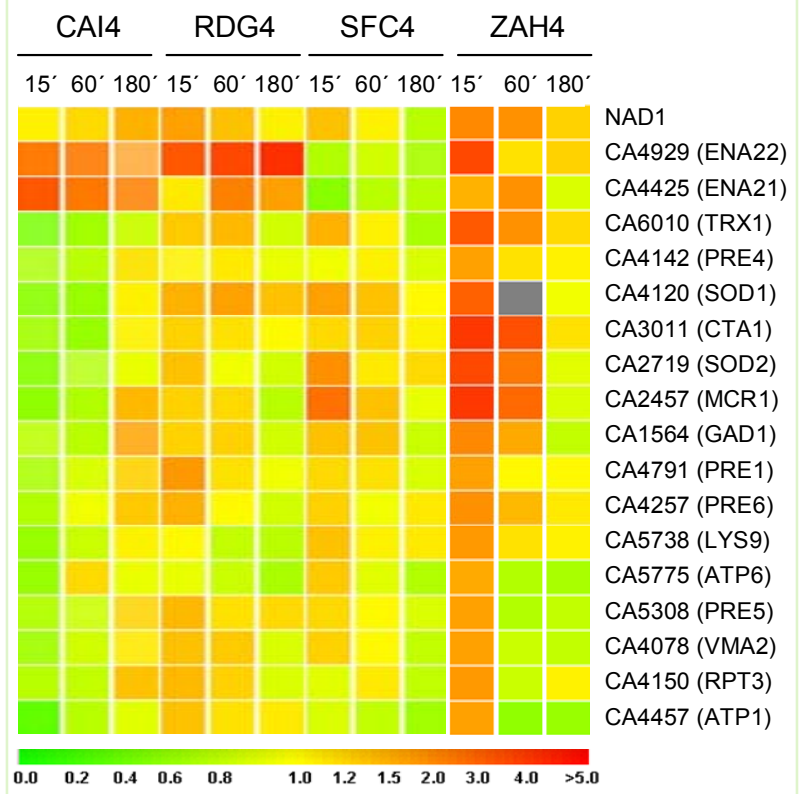

Figure 3.22 Cluster analysis of genes involved in oxidative stress and intracelular transporter in the CAI4, RDG4 $(g c n 5 \Delta)$, SFC4 $(\sin 3 \Delta)$ and ZAH4 $(g c n 5 \Delta, \sin 3 \Delta)$ strains during the yeast-hypha transition.

\section{Discussion}

Candida albicans is able to establish mucosal and invasive disease by means of different virulence factors of unknown regulation. The focus of our work was on understanding the possible compensatory effects of deletion in the same strain of a histone acetyltransferase (GCN5) and a component of histone deacetylase (SIN3) by analyzing the expression of genes associated with virulence and pathogenicity in $C$. albicans, such as those involved in the yeast-hypha transition. In our laboratory the GCN5 and SIN3, were deleted in a CAI4 background strain. In this work, we deleted the SIN3 gene in a gcn5 4 mutant background strain. Deletions of GCN5 and SIN3 appeared to block hyphal formation under a variety of standard inducing conditions, for example in the presence of serum or GlcNAc or during growth on Spider medium (Degano, Ph.D thesis in process; Monterola, Ph.D thesis 2002). During the yeasthypha transition, both proteins seem to suppress transcription of genes that directly or indirectly control hypha switch, which seems to be operative in both liquid and solid media assayed. On the other hand Gcn5p and $\operatorname{Sin} 3 p$ are positively required for maintenance of the yeast growth form. In the absence of Gcn5p, cells change to a pseudohypha form (Degano, Ph.D thesis in process) and loss of Sin3p caused cells change to a cigarette-like form (Monterola, Ph.D thesis 2002). Furthermore, Gcn5p and $\operatorname{Sin} 3 p$ are required for the competence of yeast cells to undergo hyphal morphogenesis in standard (aerobic) conditions. The functions of GCN5 and SIN3 on the known filamentation pathways in $C$. albicans are as yet unknown. In present work, we show 
that deletion of $C$. albicans SIN3 in a gcn5 $\Delta$ background strain compensate growth defect of the gcn5 during exponential growth in rich liquid medium at $28^{\circ} \mathrm{C}$. Morphologically, like both simple mutants, double mutant is very different to CAI4 strain and was showed an intermediate phenotype of the $g c n 5 \Delta$ and $\sin 3 \Delta$. This result suggested that in the standard condition, both proteins control independently expression of different set of genes involved in morphogenesis. The main phenotype of the double mutant is the inability to switch from the yeast to the filamentous form in liquid medium containing serum or at $37^{\circ} \mathrm{C}$ and in all the solid media assayed. The results also demonstrating that the $g c n 5 \Delta, \sin 3 \Delta$ double mutant as well as both simple mutants completely abolished chlamydospore formation. Recent studies have been shown that in C. albicans, genes like CYP56, ISW2, MDS3, RIM101, RIM13, SCH9 and SUV3 are required for efficient chlamydospore formation. Our results indicate that either deletion any of both genes mimic the effect described previously (Melo et al., 2008; Nobile et al., 2003). In the double mutant a total of 558 genes, out of 6039 present in the microarray, changed in a minimal medium (YNB) at $28^{\circ} \mathrm{C}$ during the yeast mode of growth. During the yeast growth form a number of genes involved in DNA synthesis and replication was up-regulated. One of them -RFA1- overlapped between the double and $\sin 3 \Delta$ simple mutant and recently has been implicated in cell cycle progression (Niu et al., 2008). It has been described that over-expression of RFA1 delayed the cell cycle at G2/M phases by disrupting spindle attachment to chromosomes and activating the DNA damage checkpoint (Niu et al., 2008). Morphologically the double and $\sin 3 \Delta$ simple mutants were showed a cigarette shape phenotype suggested $\operatorname{Sin} 3 p$ control cell shape through regulation of RFA1 in $C$. albicans. None of genes involved in the DNA synthesis and replication category (except of RFA1 in $\sin 3 \triangle$ ) appeared regulated in any gcn $5 \Delta$ or $\sin 3 \Delta$ simple mutants. This result suggested that this group of genes is regulated jointly by Gcn5p and $\operatorname{Sin} 3 p$ under standard condition. Upon comparing our results concerning oxidative stress genes with those described in the $\sin 3 \Delta$ simple mutant during the yeast growth form, we observed up-regulation genes involved in the oxidative stress response (Table 3.3) and some of them have been regulated in the $\sin 3 \Delta$ simple mutant consistent with the phenotype of both strains ( $\sin 3 \Delta$ and double mutant) in a medium containing hydrogen peroxide $\left(\mathrm{H}_{2} \mathrm{O}_{2}\right)$. Furthermore, the up-regulation of genes involved in DNA polymerase and DNA repair including CDC21 (Liu et al., 2005), DPB2 (Backen et al., 2000), RAD51 (Liu et al., 2005) and RAD6 (Leng et al., 2000) maybe play a role in DNA repair, by bypassing DNA damage caused by hydroxyl radicals $(\mathrm{OH})$, are also in agreement with resistance phenotype of the double and $\sin 3 \Delta$ simple mutants to hydrogen peroxide 
$\left(\mathrm{H}_{2} \mathrm{O}_{2}\right)$. Loss of GCN5 and SIN3 results in the up-regulation of numerous genes involved in glycolysis and TCA cycle. The metabolism of glucose to pyruvate during glycolysis produces energy in the form of ATP (Pile et al., 2002). Of the ten enzymes in the glycolytic pathway, four are transcriptionally up-regulated in the double mutant. In addition, the expression of genes involved in tricarboxylic-acid cycle also was upregulated (Table 3.3) indicate a metabolic change in the double mutant. Several authors have been described the up-regulation genes involved in citric acid and glyoxylate cycles under many different conditions, for example in the APSES mutants and Drosophila sin3 $\Delta$ mutant cells (Doedt et al., 2004; Pile et al., 2003). Deletion of GCN5 and SIN3 also results in the up-regulation a number gene involved in fatty acid oxidation (ECI1 and POX1-3). Thus, under standard conditions GCN5 and SIN3 jointly could act as transcriptional regulators of genes playing roles in glycolysis, fatty acid oxidation and the citric acid cycle. We found at least, that nineteen genes corresponding to well known cell wall proteins were regulated during the yeast mode of growth in the double mutant (Table 3.3) suggesting a cell wall remodelling. Phenotype analysis of the double mutant in medium containing Caffeine, Congo red, $\mathrm{NaCl}$, SDS and Zymolyase confirms that the double mutant cells undergo a change in cell wall composition (Fig. 3.7 and 3.8). Another group of regulated genes included sixteen genes coding for transcription factors (Table 3.3). None of them are regulated in the gcn5 $\triangle$ and $\sin 3 \Delta$ simple mutants except RIM101, PPR1 and LEU3 that have been reported to be up-regulated in the gcn5 $\Delta$ simple mutant (Degano, Ph.D thesis in process). Six of them have been characterized as transcription factors with a zinc cluster DNA-binding motif (Park et al., 2003) and the rest belong to disperse family of regulators that respond to different factors and regulate a variety of genes with different functions. Our results suggest that under standard condition Gcn $5 p$ and $\operatorname{Sin} 3 p$ jointly regulate expression a number of transcriptional regulators. During the hypha transition, a total of 1271 genes, out of 6039 present in the microarray, changed due to deletion GCN5 and SIN3, in a minimal medium (Lee) at one of the three incubation times assayed $(15,60$ and $180 \mathrm{~min})$. These represent a percentage of $21 \%$ of $C$. albicans genome indicating an important role for both genes. 35 genes encoding for transcription factors were regulated during at least one time of the yeast-hypha transition. Of those two genes including GAT2 and UME6 were down-regulated at the three times (Table 3.4) and others were regulated at one or two times of incubation (Supplementary table 3.4). GAT2 similar to S. cerevisiae Gat2p, transposon mutation affects filamentous growth (Uhl et al., 2003) and UME6 is a transcription factor; required for wild-type hyphal extension (Banerjee et al., 2008; Uhl et al., 2003). 
Deletion of two genes produces clear defects in hyphal formation and extension. Regulation of GAT2 and UME6 has been reported to be decreased in the gcn $5 \Delta, \sin 3 \Delta$ simple and in the $g c n 5 \Delta$, hda $1 \Delta$ double mutant and increased in the CAl4 strain during the hypha transition. Thus, SIN3 and GCN5 regulate specifically, in up to now an unknown way, both transcription factors. We have found regulated at least 37 genes corresponding to well characterize cell wall proteins and many genes coding for glucanases, glucosidases, mannosyltransferases and GPI anchored proteins. Some of those genes overlap with both $g c n 5 \Delta$ and $\sin 3 \Delta$ simple mutants at different time points. Different regulation of cell wall genes (e.g. ALS1, ALS3, ECM33 and SUN41) have been described by our group during induction of the morphogenetic switch by temperature in CAI4 strain and some of them were also observed in other double mutant (e.g. gcn5 ${ }^{2}$, $d a 1 \Delta$ ) suggested that the physiological reprogramming by loss of Gcn5p and Sin3p involves in remodelling of cell wall composition. We found PHR1 and PGA62 genes to be down-regulated in the gcn5 simple and double mutants at the three times of induction, while an up-regulation was described in the $\sin 3 \Delta$ and CAl4 strain in the same conditions. We have detected up- and down-regulation of genes related to the translation apparatus at $60 \mathrm{~min}$. Regulation of this group of genes have not been reported in previous works describing the yeast-hypha transition (Kadosh \& Johnson, 2005; Nantel et al., 2002; Singh et al., 2005) although it have been found in both simple mutants. We have detected a decrease in the transcript of genes involved in ergoestreol biosynthesis at $60 \mathrm{~min}$. Ergosterol genes were mainly up-regulated after exposition to antifungal agents (Liu et al., 2005; Moreno et al., 2007) and some of them has been reported to be down-regulated at $15 \mathrm{~min}$ of incubation in the gcn $5 \Delta$, hda $1 \Delta$ double mutant and CAI4 strain suggesting that both mutations do not affect directly the expression of those genes. We have detected down-regulation genes involved in cell polarity (e.g. CDC42, GPA2 and GPR1) and signal transduction at $60 \mathrm{~min}$ of incubation. Several authors have been described changes of genes involved in cell polarity and signal transduction under hypha development and described that expression some of genes like CDC42 is required for budding and maintenance of hyphal growth (Bassilana et al., 2005; Mirbod et al., 1997; Ushinsky et al., 2002), GPA2 that regulates filamentous growth (Miwa et al., 2004; Sanchez-Martinez \& PerezMartin, 2002), GPR1 required for wild-type hyphal growth (Maidan et al., 2005; Miwa et al., 2004; Prigneau et al., 2003). It has been reported that expression most of them to be increased in CAI4 strain and decreased in both simple mutants. Thus our result is in agreement with phenotype of double mutant under the morphogenetic switch. No previous data have been reported for the other sets of genes described in this Chapter; 
namely, those coding for vitamin biosynthesis, genes of the proteasome subunits, genes involved in peroxisomal and for the $F_{1} F_{0}$ ATPase complex. 
Los hongos son organismos ubicuos encontrándose especies fúngicas en casi todos los hábitats. Muchas de estas especies son capaces de adaptarse a diferentes ambientes, desarrollando en un corto periodo de tiempo una respuesta transcripcional y fisiológica específica ante un cambio de las condiciones del medio (Berman, 2004). El hecho de que los hongos sean organismos eucariotas con un desarrollo rápido y que resulten más fáciles de manipular que los organismos pluricelulares, ha estimulado la utilización de algunas especies de levaduras como organismos modelo en estudios genéticos y de biología molecular. Especialmente este es el caso de Saccharomyces cerevisiae, sin embargo, esta especie no es patógena, por lo que no resulta útil a la hora de analizar mecanismos de virulencia, ni presenta la plasticidad morfológica característica de otras especies fúngicas. Un hongo que resulta de gran utilidad como modelo para analizar ambos tipos de procesos es Candida albicans.

\section{Candida albicans}

Se trata de un patógeno fúngico oportunista de alta incidencia en humanos, sobre el que es necesario realizar nuevos trabajos de investigación, con el fin de desarrollar tratamientos terapéuticos eficaces. Estos conocimientos, además pueden ayudar a comprender la biología molecular de otros patógenos fúngicos más virulentos cuyo manejo en laboratorio resulta complicado. C. albicans es capaz de crecer adoptando diferentes morfologías, según las condiciones del medio, por lo que resulta útil como modelo en estudios de diferenciación celular. Tradicionalmente se ha clasificado a $C$. albicans como un hongo dimórfico por su capacidad de intercambiar la morfología de hifa y la de levadura. Al igual que otras especies englobadas dentro de este grupo es capaz de adoptar más de dos morfologías, por lo que debería considerarse en realidad como un hongo polimórfico. En España los datos obtenidos entre 1990 y 1999 indican que los agentes fúngicos provocan entre el $4.5 \%$ y el $7 \%$ de las infecciones totales, siendo $C$. albicans el principal patógeno fúngico. Esta especie se encuentra entre los 10 microorganismos más frecuentes del total de aislamientos tanto de infecciones comunitarias como nosocomiales (estudio EPINE, proyecto sobre la "Evolución de la Prevalecía de las infecciones nosocomiales en los hospitales españoles", 1990-1999). Según un estudio publicado recientemente, la tasa de mortalidad asociada a candidemias sistémicas en España se encuentra en $40.6 \%$, valor similar al estimado en otros países (Peman et al., 2005; Peman, 2005). En todos los estudios realizados se ha puesto de manifiesto que aunque la mayor parte de las candidiasis se deben a $C$. 
albicans, en los últimos años se ha detectado un aumento en la incidencia de otras especies del género, especialmente C. parasiliensis y C. krusei (Ruiz, 2004). Estos datos son importantes dado que la especie que se ha asociado con mayor mortalidad y que presenta mayor resistencia a los antifúngicos de elección, los azoles, es C. krusei (60\% en seguimientos realizados en España) (Peman et al., 2005).

\section{Características generales y posición taxonómica}

El género Candida engloba unas doscientas especies que comparten características como reproducción asexual por gemación y ausencia de pigmentos carotenoides. Las especies del género Candida se incluyeron inicialmente dentro de la clase Deuteromycetes, grupo que incluye los comúnmente llamados "hongos imperfectos" y que se corresponden con especies no relacionadas cuya principal característica es la ausencia de ciclo sexual conocido. Actualmente el género Candida se ha incluido dentro de los ascomicetos. Siguiendo recientes clasificaciones (Calderone, 2002): el género Candida se encuadra dentro del Reino Fungi, Phylum Ascomycota, Clase Ascomycetes, Orden Saccharomycetales, Familia Candidaceae. La comparación de secuencias de diferentes genes indican que filogenéticamente el grupo más próximo evolutivamente es el de los ascomicetos. Respecto a $C$. albicans, en los últimos años se han publicado diferentes trabajos sobre la posible existencia de un ciclo sexual en esta levadura (Hull \& Johnson, 1999; Hull et al., 2000; Magee \& Magee, 2000; Magee \& Magee, 2004; Soll et al., 2003). Las características más destacadas de C. albicans son: su capacidad de formar tubos germinativos y clamidosporas, seguida de su patrón de asimilación de azúcares. Se han establecido varios biotipos dentro de la especie basándose en similitudes fenotípicas y de ADN (Odds, 1988).

\section{Características de las diferentes morfologías}

C. albicans es un organismo polimórfico, ya que es capaz de crecer en forma de hifa, levadura, pseudohifa o dar lugar a la formación de clamidosporas (Fig. 1.2). Las levaduras o blastosporas tienen forma elipsoidal con un diámetro aproximado de $5 \mu \mathrm{m}$, y se dividen por gemación. Las pseudohifas consisten en cadenas de células alargadas que se originan mediante un proceso de gemación en el que no se llegan a separar la célula madre y la célula hija. A diferencia de las hifas, las paredes laterales de las pseudohifas no se mantienen paralelas, por lo que muestran valores de diámetro variable: son más gruesas (el diámetro mínimo se encuentra alrededor de $2.8 \mu \mathrm{m}$ ) y presentan constricciones en los septos (Odds, 1988; Sudbery et al., 2004). Las clamidosporas son esporas asexuales con una pared gruesa que se forman mediante 
el recubrimiento de las células preexistentes, tienen forma redondeada con un tamaño aproximado de 8 a $12 \mu \mathrm{m}$ de diámetro y aparecen en posiciones laterales o terminales de hifas y pseudohifas, sobre células suspensoras alargadas (Fig. 1.2). In vivo rara vez se detectan estas estructuras (Cole et al., 1991). En el laboratorio su formación se induce en medios sólidos pobres en nutrientes, suplementados con detergentes, en condiciones microaerófilas y a temperaturas entre $20^{\circ} \mathrm{C}$ y $30^{\circ} \mathrm{C}$, mientras que su formación se reprime en presencia de glucosa (Calderone, 2002; Nobile et al., 2003).

\section{El genoma de Candida albicans}

C. albicans es un organismo diploide. Desde el año 2000 se dispone de la secuencia completa de su genoma. La secuenciación fue llevada a cabo por el Stanford Genome Center partiendo de ADN de la cepa SC5314. Dicha estirpe fue elegida por ser un aislado clínico usado habitualmente en análisis moleculares y ensayos de virulencia y poseer un cariotipo estándar. Se ha estimado que el genoma haploide de $C$. albicans tiene una longitud de aproximadamente $16 \mathrm{Mb}$, siendo por tanto un $33 \%$ mayor que el de S. cerevisiae. Se ha publicado el ensamblaje 20 , en el que se han podido anillar casi en su totalidad las secuencias de los supercontigs del ensamblaje 19 en los 8 cromosomas de $C$. albicans. El ADN mitocondrial de esta especie ha sido también secuenciado, estimándose un tamaño total de 40420 pares de bases (Número de accesión GeneBank: AF285261). A partir del ensamblaje 19 varias organizaciones llevaron a cabo una anotación de las posibles fases de lectura abierta (ORFs) presentes en su genoma. Se identificaron 6244 ORFs. Se ha propuesto la función de algunos de esos genes basándose en un elevado porcentaje de identidad con genes previamente caracterizados de $S$. cerevisiae, que corresponderían a sus homólogos funcionales.

\section{Relación entre morfología y ciclo celular}

El estudio detallado de las características de las diferentes morfologías de C. albicans ha permitido determinar que la organización del ciclo celular de las hifas difiere sustancialmente del de pseudohifas y levaduras. Tanto en levaduras como en pseudohifas, antes de que aparezca la yema se forma un anillo de septinas en el sitio de gemación, marcando la posición del cuello entre la célula madre y la célula hija. Durante la mitosis, la división nuclear ocurre en el plano delimitado por este anillo, y una vez completada el anillo se divide en dos, formándose entre ambos el septo primario. En ambas morfologías la división celular supone la aparición y constricción de 
un anillo de actomiosina. El ciclo celular de pseudohifas difiere del de levaduras en la mayor duración de la fase G2, lo que permite una mayor elongación de la yema, y en el mantenimiento de la unión celular tras la formación del septo (Sudbery, 2001). En el desarrollo inicial de las hifas se forma una banda basal de septinas que se dispone de forma longitudinal al tubo germinativo (Sudbery et al., 2004; Sudbery, 2001). A diferencia de los anillos de septinas de los septos, la banda basal no contiene la septina CaCdc3p y en su formación no interviene CaGin4p (Wightman et al., 2004). El núcleo de la célula madre migra hacia el interior del tubo y es allí donde se divide (Gow et al., 1986; Warenda \& Konopka, 2002). A lo largo de la hifa aparecen sucesivos anillos de septinas, organizando los septos de separación celular. A diferencia de lo que ocurre en levaduras, en las hifas los anillos de septinas no se desensamblan en cada ciclo (Sudbery et al., 2004)(Fig. 1.3). Se ha observado que las levaduras de $\boldsymbol{C}$. albicans pueden seguir un patrón de gemación bipolar, con la yema surgiendo en el polo opuesto a la anterior célula hija, o axial, con la yema formándose junto a la cicatriz anterior. Estos patrones están influenciados por la temperatura, por debajo de $25^{\circ} \mathrm{C}$ hay mayor proporción de células con patrón axial y a altas temperaturas hay mayor proporción de células con gemación bipolar (Berman \& Gow, 2004; Chaffin, 1984; Gale et al., 2001; Herrero et al., 1999a). Durante el crecimiento como pseudohifas, cada célula del filamento continúa su ciclo y desarrolla un patrón de gemación unipolar/axial. La célula hija gema en el polo opuesto al de su punto de nacimiento por lo que se forma una estructura ramificada. Durante los primeros ciclos se mantiene la división sincrónica entre las células del filamento (Gimeno et al., 1992; Sudbery et al., 2004; Yokoyama \& Takeo, 1983). El crecimiento en forma de hifa tiende a ser por tanto más linear y los patrones de ramificación tienden a ser menos regulares que los de pseudohifas (Barelle et al., 2003; Gow, 1997; Sudbery et al., 2004; Whiteway \& Oberholzer, 2004).

\section{Tipos de patologías causadas por C. albicans}

C. albicans se encuentra como comensal inocuo en la cavidad oral y los tractos digestivo y genital de humanos y otros mamíferos. Habitualmente se encuentra en equilibrio con la microbiota normal y con el sistema inmune del hospedador. Aunque altamente adaptada como organismo comensal, esta especie presenta un gran número de elementos virulentos que ante determinados estímulos le permiten colonizar y producir daños directos en el hospedador (Hube \& Naglik, 2001). Las patologías que C. albicans puede causar se engloban dentro de tres tipos: cutáneas, mucosas y sistémicas. La candidiasis cutánea se desarrolla generalmente a partir de una 
alteración de la barrera de la piel. Existen varios tipos, el intertrigo, la onicomicosis (infecciones de las uñas y el tejido circundante) y el granuloma candidiásico (Martinez Roig, 2003). Las candidiasis mucosas pueden afectar a la mucosa oral (muguetis bucal), a la esofágica (esofagitis candidiásica), a la intestinal o a la genital. Las infecciones sistémicas son las más peligrosas, se producen por invasión de uno o varios órganos internos, afectando fundamentalmente a individuos inmunocomprometidos.

\section{Factores de virulencia de Candida allbicans}

El espectro de afecciones causadas por $C$. albicans es más amplio que el de la mayoría de patógenos oportunistas. Esto es atribuible a la enorme plasticidad de su fenotipo que le permite sobrevivir en diferentes tejidos del hospedador (Calderone \& Fonzi, 2001). Nuestros conocimientos sobre la biología de $C$. albicans como comensal son muy reducidos, tan sólo se ha descrito que pequeñas variaciones en el sistema inmune del hospedador son capaces de alterar la forma de crecimiento, por lo que la línea que separa la forma comensal de la patógena parece ser realmente delgada. El método en el que se han basado la mayor parte de los investigadores para considerar una determinada propiedad como factor de virulencia consiste en demostrar que las cepas carentes de dicha actividad no son capaces de establecer infecciones, pero si son capaces de vivir como comensales. Las etapas sucesivas del proceso de infección y los diferentes tipos de patologías causados por $C$. albicans están condicionadas por diferentes combinaciones de estos factores de virulencia (Naglik et al., 2003).

\section{Rutas de señalización celular}

La supervivencia de $C$. albicans en el cuerpo humano, se comporte como comensal o como patógeno, depende no sólo de su capacidad de respuesta, sino también de su capacidad para detectar y transmitir los estímulos del ambiente exterior. Las rutas de señalización celular son uno de los mecanismos celulares más conservados en todos los organismos (Lengeler K.B., 2000; Lengeler et al., 2000). Están formadas por una sucesión de proteínas que se van activando unas a otras en cascada. Una característica importante es la regulación transversal entre ellas para asegurar que no son activadas por la señal errónea, ya que algunas de las proteínas. El cambio entre la forma de crecimiento de levadura y la de hifa puede considerarse una respuesta adaptativa por la que determinados hongos ajuntan su morfología a una nueva situación ambiental. Se desarrolla por la sucesión de una serie de procesos 
encadenados: percepción de un condicionante externo, traducción de la señal en un mensaje bioquímico, expresión génica diferencial y desarrollo de un patrón de crecimiento polarizado (Chio, 2004 )(Fig. 1.5).

\section{Rutas de transducción de señales de la transición dimórfica}

Existen múltiples rutas de señalización en $C$. albicans que regulan la transición levadura-hifa. Entre las que destacan 4 vías que inducen la formación de hifas: la ruta de proteínas quinasas activada por mitógeno vía CaCph1p (ruta MAPK), la ruta de la proteína quinasa A dependiente de cAMP mediada por CaEfg1p (ruta PKA-CAMP), la ruta de respuesta a $\mathrm{pH}$ a través de CaRim101p y la ruta de respuesta a una matriz extracelular mediada por CaCzf1p. Existen además varias vías represoras de la filamentación que actúan a través de CaTup1p o bien a través de CaRbf1p (Fig. 1.6).

\section{- Ruta de proteínas quinasas activada por mitógeno (ruta MAPK)}

En S. cerevisiae la ruta MAPK ( respuesta a feromonas, esta implicada en el proceso de conjugación, en el desarrollo de pseudohifas y en el crecimiento invasivo. En $C$. albicans se han identificado proteínas homólogas a las que conforman esta ruta en $S$. cerevisiae y se ha comprobado que están relacionadas con filamentación bajo condiciones de limitación de nutrientes (Ernst, 2000b).

\section{- Ruta de la proteína quinasa A dependiente de cAMP (ruta PKA-cAMP)}

La ruta PKA-cAMP juega un papel crucial en el control de la filamentación en $C$. albicans, S. cerevisiae y otros hongos. En C. albicans la actividad de esta ruta es necesaria para la filamentación bajo múltiples estímulos, como la carencia de nutrientes o la presencia de inductores químicos como la NAcGlc o el suero (Chio, 2004 ; Ernst, 2000b).

\section{- Ruta de inducción por pH}

En C. albicans el cambio de $\mathrm{pH}$ ácido a neutro induce la formación de hifas a $37^{\circ} \mathrm{C}$. Este organismo posee una ruta de respuesta a $\mathrm{pH}$ conservada con respecto a otros hongos. Al igual que en S. cerevisiae y Aspergillus nidulans, el factor de transcripción CaRim101p (CaPrr2p) regula la expresión de genes de respuesta a $\mathrm{pH}$, al ser activado por proteolisis dependiente de la proteínas CaRim8p (CaPrr1p) y CaRim20p (CaEnx3p) 
La capacidad de C. albicans de filamentar cuando crece dentro de una matriz sólida se considera una respuesta que facilita la penetración del hongo en los tejidos del hospedador. Se ha identificado una ruta que induce la formación de hifas en estas condiciones, en la que participa la proteína CaCzf1p. Esta proteína contiene un dominio "Zinc finger", generalmente implicado en la interacción con secuencias de ADN. El gen CaCZF1 no es necesario para la respuesta a suero, pH, NAcGlc, prolina o medio Spider pero si es necesario para la correcta filamentación en medio embedded, por lo que se ha relacionado con la respuesta al estrés mecánico provocado por el crecimiento dentro del agar (Brown et al., 1999) y con la respuesta a una baja concentración de oxígeno que viene provocada por esta forma de crecimiento (Ernst, 2000a).

\section{- Regulación negativa de la filamentación}

CaTUP1 codifica un factor transcripcional determinante en el mantenimiento del crecimiento en forma de levadura. La deleción de CaTUP1 provoca crecimiento en forma de pseudohifas en todos los medios ensayados, tanto en condiciones inductoras como no inductoras de la miceliación (Braun \& Johnson, 1997). En S. cerevisiae, la proteína ScTup1p interacciona de forma indirecta con los promotores de determinados genes, a través de su unión a diferentes proteínas de unión a ADN (Smith \& Johnson, 2000). Una de las proteínas de S. cerevisiae capaces de interaccionar con ScTup1p es ScNrg1p, que es un factor transcripcional con un dominio de "Zinc finger" de unión a $A D N$, que dirige el complejo ScSsn6p-ScTup1p a determinados genes diana. En $C$. albicans se ha demostrado que la transcripción de CaNRG1 es menor durante la formación de las hifas en suero a $37^{\circ} \mathrm{C}$ (Braun et al., 2001; Liu, 2001) y que la inactivación de esta proteína causa crecimiento filamentoso e invasivo en condiciones no inductoras. A diferencia del mutante Catup1, el mutante Canrg1 es capaz de formar hifas auténticas en condiciones de inducción (Murad et al., 2001b). La asociación con CaTup1p parece clara, ya que se ha demostrado que CaNrg1p reprime la expresión de genes relacionados con virulencia y morfogénesis que son también reprimidos por CaTup1p y que la mayoría de ellos contienen en su promotor un elemento NRE (Nrg1 response element) (Murad et al., 2001b). 
Las cuatro histonas $(\mathrm{H} 2 \mathrm{~A}, \mathrm{H} 2 \mathrm{~B}, \mathrm{H} 3$ y $\mathrm{H} 4)$ que constituyen los nucleosomas poseen un dominio globular y uno o dos dominios amino-terminales flexibles, que junto con la región carboxilo terminal de la histona $\mathrm{H} 2 \mathrm{~A}$, median las interacciones internucleosomales, favoreciendo la compactación del ADN (Wolffe \& Guschin, 2000). En los dominios terminales de las histonas se encuentran residuos de lisinas susceptibles de ser acetilados de forma reversible. Estos aminoácidos se encuentran en posiciones muy conservadas en todos los organismos eucariotas, especialmente en las histonas $\mathrm{H} 3$ y H4. El estado de acetilación es regulado por la acción opuesta de dos tipos de enzimas, las histonas acetiltransferasas (HATs) y las histonas desacetilasas (HDACs). Actualmente se sabe que algunas de estas enzimas son capaces de actuar sobre otras proteínas diferentes de las histonas (Yang \& Gregoire, 2005). Se ha sugerido que los grupos acetilo (cargados negativamente) contrarrestan la carga positiva de los residuos de lisina y por tanto disminuyen la interacción de las histonas con el ADN (cargado negativamente) del propio nucleosoma y de nucleosomas próximos. De esta forma se facilita el acceso de la maquinaria de transcripción general y de proteínas reguladoras. Siguiendo este modelo, la desacetilación tendría el efecto contrario y ya que al aumentar el grado de empaquetamiento se dificultaría la transcripción (Fig. 1.8) (Kurdistani \& Grunstein, 2003). De forma alternativa se ha sugerido que el efecto de la acetilación sobre la transcripción podría deberse, no a modificaciones de la estructura de la cromatina, sino a que los residuos acetilados proporcionan superficies de anclaje apropiadas para determinados componentes de la maquinaria de transcripción u otros reguladores de la expresión génica (Kurdistani \& Grunstein, 2003). Las histonas acetiltransferasas (HATs) y las histonas desacetilasas (HDACs) actúan junto a otras proteínas formando complejos multiproteicos. Se han detectado interacciones de algunos de estos complejos con proteínas de unión a ADN que dirigen las HDACs y las HATs a determinadas secuencias de los promotores, actuando sobre los nucleosomas adyacentes al sitio de unión. Este modo de acción se ha denominado acetilación-desacetilación específica (Kurdistani \& Grunstein, 2003; Vogelauer et al., 2000). Los enzimas histonas acetiltransferasas y también las histonas desacetilasas, in vivo forman parte de complejos multiproteicos de elevada masa molecular. Los complejos HAT se han clasificado en dos tipos A y B en función de su localización subcelular y de su habilidad para modificar o no las histonas. Las HATs tipo A son enzimas nucleares capaces de acetilar histonas ensambladas en cromatina. 
Los complejos HAT tipo B se han descrito como complejos de localización preferente en el citoplasma que únicamente acetilan histonas libres, probablemente para su posterior deposición en cromatina.

\section{Características del gen GCN5}

El co-activador transcripcional GCN5 (general control nonrepressed) se ha descrito como HAT de tipo $B$, una histona acetiltransferasa por su similitud con la proteína p55, un activador HAT nuclear aislado de Tetrahymena (Georgakopoulos, 1992). Estudios genéticos sobre GCN5 habían puesto de manifiesto su función en la regulación transcripcional de algunos genes, por tanto, el descubrimiento de la capacidad de acetilar histonas de esta proteína supuso un nexo importante entre la acetilación de histonas y la regulación transcripcional. La lisina 14 de H3 es una de las principales dianas de acetilación de Gcn5p (Kuo et al., 1996; Kuo \& S. Y. y Allis, 1996), aunque su especificidad varía dependiendo de las proteínas que le acompañen. Hasta el momento se ha identificado que Gcn5p es la subunidad catalítica de al menos tres complejos tipo A diferentes de levadura: ADA (0.8 MDa), SAGA (1.8 MDa) y HAT-A2 (180 kDa) (Carrozza, 2003). Gcn5 forma la subunidad catalitica junto a Ada2 y Ada3. Gcn5 libre es capaz de acetilar histonas libres preferemente en la lisina 14 de $\mathrm{H} 3$, mientras que en el complejo Ada, acetila las lisinas 9, 14 y 18 de esa misma histona (Grant et al., 1997).

\section{Características de los genes HAT1 y HAT2}

La proteína Hat1p de levadura fue la primera proteína de tipo HAT identificada y fue descrita como HAT de tipo B. Las proteínas HATs de tipo B usan histonas recién sintetizadas como sustratos; esas histonas son acetiladas antes de ser transportadas al núcleo. Hat1p se purifica por tanto en las fracciones citoplasmáticas junto con Hat2p, un homólogo de RbAp48 (una proteína asociada $\mathrm{a} \mathrm{Rb}$ ) que actúa como una posible chaperona de la histona $\mathrm{H} 4$ y es también una subunidad del factor 1 de ensamblaje de la cromatina (CAF1), lo que refuerza la idea de la relación entre la actividad acetiltransferasa de Hat1p y el ensamblaje de la cromatina. RbAp48 también interactúa con subunidades catalíticas de enzimas desacetilasas. Esto sugiere que RbAp48 dirige el tetrámero acetilado de histonas $\mathrm{H} 3 / \mathrm{H} 4$ hacia las horquillas de replicación del ADN durante el proceso de replicación. Además, los complejos de desacetilación también son dirigidos, probablemente vía interacción con homólogos de RbAp48, hacia los nucleosomas recién ensamblados eliminando grupos acetato (Kuo \& Allis, 1998). Así, Hat1p y Hat2p forman un complejo de acetiltransferasas de histonas de tipo $B$, donde Hat1p es la subunidad catalítica de la enzima que acetila específicamente los residuos 
de lisina de las posiciones 5 y 12 de las histona $\mathrm{H} 4$ libre y Hat2p es la subunidad regulatoria que aumenta la actividad de Hat1p promoviendo la interacción entre Hat1p y el extremo amino terminal de la histona H4 (Qin \& Parthun, 2002). Se ha demostrado que la acetiltransferasa Hat1p participa en el silenciamiento de los telómeros. El silenciamiento de los telómeros implica una represión transcripcional de los genes próximos a estas regiones (los genes subtelomérico) y está mediado por la formación de heterocromatina. Cuando se delecionan los genes HAT1 o HAT2 no se observa ningún cambio en el nivel de silenciamiento de los genes subtelomérico pero sí se observa un defecto significativo en el silenciamiento cuando las mutaciones en los genes HAT1 y HAT2 se combinan con mutaciones en el extremo amino terminal de la histona $\mathrm{H} 3$. En la histona $\mathrm{H} 4$, el papel de Hat1p en el silenciamiento subtelomérico está mediado exclusivamente por la lisina 12. Además, en contraste con otras acetiltransferasas la actividad Hat1p es necesaria para la represión transcripcional más que para la activación génica (Kelly et al., 2000a). El papel de la proteína Hat1p se ha relacionado con la reparación del ADN debido principalmente a dos observaciones: en primer lugar, algunos mecanismos de reparación de daño en el ADN requieren la síntesis de nuevo ADN, que necesita ser ensamblado en la cromatina y, en segundo lugar, se ha descubierto que algunos factores reguladores de levaduras afectan tanto al silenciamiento de los telómeros como a la reparación del daño en el ADN. Así, se ha demostrado que la proteína Hat1p y residuos específicos de lisina en el extremo amino terminal de la histona H3 están implicados en la reparación del daño en el ADN. Concretamente, se ha visto que la histona H3 y la proteína Hat1p contribuyen a la reparación por recombinación de las roturas en el ADN de doble cadena (Qin \& Parthun, 2002).

\section{Características del gen SIN3}

La proteína $\operatorname{Sin} 3 p$ de diferentes especies constituye la plataforma sobre la cual se ensambla un complejo con actividad histona desacetilasa denominado complejo SIN3/HDAC. En mamíferos, el núcleo del complejo está formado por 8 proteínas mSin3, RbAp46, RbAp48, Sap30, Sap18, Sds3 y las histona desacetilasas HDAC1 y HDAC2. En S. cerevisiae, hasta el momento, sólo se ha detectado interacción de ScSin3p con una histona desacetilasa, ScRpd3p, por lo que el complejo se conoce como Sin3/Rpd3. Sin embargo, no se descarta que ScSin3p pueda interaccionar con otras histona desacetilasas (Kadosh \& Struhl, 1997; Rundlett et al., 1998; Silverstein \& Ekwall, 2005). Dentro del complejo de S. cerevisiae se aislaron otras proteínas como ScSap30p, ScSds3p, ScPho23p y ScUme6p (Kurdistani \& Grunstein, 2003) (Fig. 4.1). 


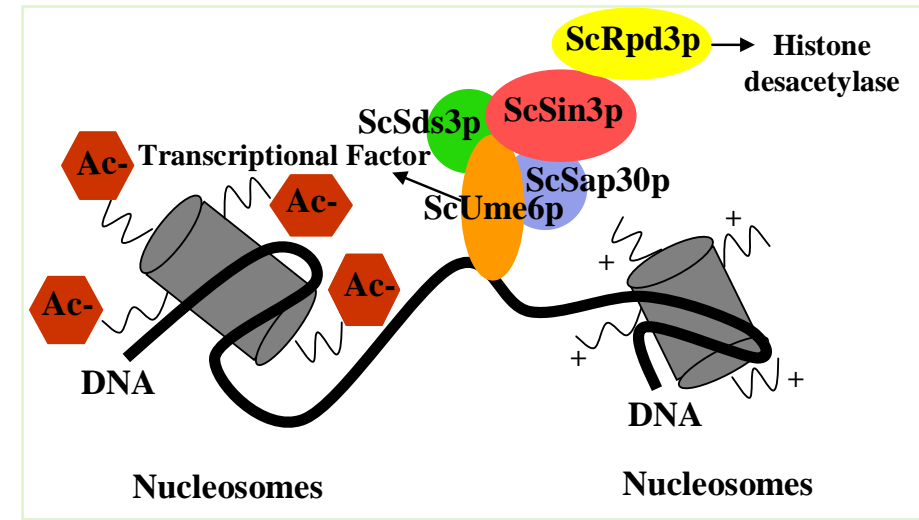

Figura 4.1 Esquema del complejo de Sin3/Rpd3 en S. cerevisiae

Se ha observado que la proteína $\operatorname{ScSin} 3 p$ no es necesaria para la actividad histona desacetilasa in vitro pero si in vivo, probablemente debido al papel de ScSin3p en dirigir a ScRpd3p a determinados promotores (Wu et al., 2001). En nuestro laboratorio por la Dra. Monterola se ha caracterizado el gen de C. albicans homólogo al gen SIN3 de $S$. cerevisiae y se ha analizado su papel en morfogénesis. A $28^{\circ} \mathrm{C}$, las células carentes de $\sin 3 \Delta$ son más alargadas que las de la cepa CAl4 y en todos los medios de inducción de la filamentación ensayados, la cepa delecionada no es capaz de formar auténticas hifas, sólo pseudohifas. En el mismo trabajo fue aislado el gen SIN3 de otra levadura dimórfica, Yarrowia lipolytica, observándose que, al igual que en C. albicans, la cepa delecionada no era capaz de formar hifas (Monterola, 2002; Tebarth et al., 2003). La ausencia del gen SIN3 de C. albicans altera la expresión de un factor clave en las rutas de señalización de la transición morfológica, CaEfg1p. En las cepas silvestres de $C$. albicans, el nivel de expresión de EFG1 disminuye rápidamente en los primeros minutos tras la inducción de la filamentación y a continuación va aumentando progresivamente. En el mutante nulo $\sin 3 \Delta$ la expresión se mantiene constante, de forma que durante las dos primeras horas de la transición dimórfica el nivel de expresión de EFG1 es mayor en la cepa delecionada que en la cepa silvestre. Se ha demostrado que CaSin $3 p$ es capaz de interaccionar con el promotor del gen EFG1 y que en la represión de EFG1 interviene la propia proteína CaEfg1p. Considerando estos datos, junto con la observación de que el fenotipo mostrado por la deleción de SIN3 es similar al de la sobreexpresión de EFG1, se ha propuesto que CaSin3p interviene en la autorregulación negativa de EFG1 (Monterola, 2002; Tebarth et al., 2003). 


\section{Objetivos}

La capacidad de $C$. albicans de llevar a cabo la transición levadura-hifa es una de sus características más estudiadas, ya que se ha visto que las hifas están implicadas en procesos de virulencia y que cepas incapaces de llevar a cabo la formación de hifas son avirulentas (Lo et al., 1997; Calderone, 2002). Nos planteamos los siguientes objetivos:

1. Determinar, bajo diferentes condiciones de inducción, si las histonas acetiltransferasas CaHat1p y CaHat2p, en el doble mutante de $C$. albicans, son necesarias para el desarrollo de procesos morfogenéticos, tales como la formación de hifas, pseudohifas y clamidosporas.

2. Determinar el sitio de comienzo del transcrito en los genes HAT1 y HAT2.

3. Determinar la localización subcelular de las proteínas Hat1p y Hat2p.

4. Comprobar si la deleción de los genes hat1 $\Delta$ y hat2 $\Delta$ en los mutantes simples y en un doble mutante afectan a la virulencia de $C$. albicans en un modelo de infección sistémica de ratón.

5. Analizar el transcriptoma de los mutantes hat $2 \Delta$ y del doble mutante hat $1 \Delta$, hat $2 \Delta$ durante el crecimiento levaduriforme y durante en el proceso de transición levadura-hifa.

6. Determinar la participación de CaGcn5p y CaSin3p en el doble mutante ZAH4, en la regulación de la transición levadura-hifa en diferentes condiciones de inducción, así como en la formación de clamidosporas.

7. Analizar el transcriptoma del doble mutante $g c n 5 \Delta, \sin 3 \Delta$ durante el crecimiento en fase exponencial a $28^{\circ} \mathrm{C}$ y durante la transición levadura-hifa a $37^{\circ} \mathrm{C}$.

8. Comprobar si la deleción de los genes $\sin 3 \Delta$ como mutante simple y gcn $5 \Delta$, $\sin 3 \Delta$ doble mutante afectan a la virulencia de $C$. albicans en un modelo de infección sistém 


\section{Medios de cultivo y condiciones de crecimiento}

\section{Medios de cultivo para C. allbicans}

En la Tablas 2.1 y 3.1 se muestran los genotipos más relevantes de las cepas de $C$. albicans utilizadas y obtenidas en el presente trabajo. Los medios de cultivo empleados para el crecimiento de levaduras fueron: medio YEPD y YNB. Para los medios de cultivo sólidos añadiendo agar al $2 \%$. Los cultivos en medio líquido de $C$. albicans se realizaron en matraces Erlenmeyer. El volumen del medio fue siempre inferior a un tercio del volumen del matraz. Las incubaciones se llevaron a cabo en agitadores orbitales a $200 \mathrm{rpm}$ y a $28^{\circ} \mathrm{C}$. El crecimiento se determinó midiendo la absorbancia en un espectrofotómetro (Hitachi modelo U-2001) a una longitud de onda de $600_{\mathrm{nm}} \mathrm{O}$ mediante el recuento de células en una cámara Thoma. De forma rutinaria, para los inóculos se estimó la cantidad de células considerando la correspondencia entre un valor de D.O de 0.4 con una concentración de $10^{7}$ células $/ \mathrm{ml}$.

En todos los casos las células en los medios sólidos utilizados para la inducción de crecimiento filamentoso se sembraron a razón de 50 ufc por placa y se incubaron a $37^{\circ} \mathrm{C}$, excepto en el caso del medio Embedded en el que la suspensión de células se mezcló con este medio previamente mantenido a $50^{\circ} \mathrm{C}$. A continuación se sirvió en placas, se dejaron solidificar y se incubaron a $28^{\circ} \mathrm{C}$.

El medio empleado para la inducción de clamidosporas fue cornmeal agar al $1.7 \%$ (Difco). Después de disolver y autoclavar el medio se añadió Tween 80 (Sigma) hasta una concentración final del $0.33 \%$. Las células de las diferentes cepas de C. albicans se sembraron en estrías y sobre ellas se dispusieron cubreobjetos con el fin de crear condiciones de crecimiento microaerófilas. Las placas se incubaron en oscuridad a $25^{\circ} \mathrm{C}$, durante 7-14 días y se tomaron muestras de cada una de ellas. Para mejorar la visualización de las clamidosporas al microscopio óptico, las muestras se sometieron a una tinción con lactofenol-azul algodón.

Las cepas obtenidas y empleadas en el presente trabajo se conservaron mediante resiembras periódicas en placas de medio sólido. Las placas inoculadas se incubaron a $28^{\circ} \mathrm{C}$ durante 48 horas en el caso de C. albicans y a $37^{\circ} \mathrm{C}$ durante 12 horas en el caso de E. coli. Posteriormente, se conservaron a $4^{\circ} \mathrm{C}$. Con la finalidad de mantener las células durante largos periodos del tiempo, los microorganismos se almacenaron en viales con glicerol al $30 \%$ en ultra congeladores a $-80^{\circ} \mathrm{C}$.

Dado que en todos nuestros experimentos de transformación se utilizó el gen CaURA3 como marcador de selección, las cepas a transformar fueron siempre Ura (CAl4 y derivadas). 


\section{Medio de cultivo para E. coli}

Las bacterias se crecieron en medio LB (1\% $(\mathrm{p} / \mathrm{v})$ bactotriptona, $0.5 \%(\mathrm{p} / \mathrm{v})$ extracto de levadura, $1 \%(\mathrm{p} / \mathrm{v}) \mathrm{NaCl})$.

\section{Detección de secuencias de ADN específicas (Southern-blot)}

Una vez digerido el ADN genómico de C. albicans $(10 \mu \mathrm{g})$ con las enzimas de restricción adecuadas, los fragmentos de $A D N$ se separaron, junto a un marcador de peso molecular, mediante electroforesis en geles de agarosa al 1\%. EI ADN separado se visualizó por la incorporación en el gel de bromuro de etidio y se fotografió junto a una regla con el fin de tener una referencia que permitió, posteriormente, determinar el tamaño de las bandas hibridadas. La hibridación se realizó en una solución de igual composición a la de prehibridación y a la que se añadió la sonda radiactiva previamente desnaturalizada. La membrana (Hybond- $\mathrm{N}^{\mathrm{TM}}$ de Amersham Pharmacia Biotech) se dejó hibridando durante aproximadamente 16 horas a $65^{\circ} \mathrm{C}$. A continuación, se realizaron varios lavados de tiempo variable con $100 \mathrm{ml}$ de las soluciones de los lavados. Los filtros se analizaron con el sistema de detección de radiación, captura y análisis de imágenes BAS-1500 Storage Phosphor Imaging System (Fujifilm). Para ello cada membrana se expuso en oscuridad a una pantalla del sistema o IP (Image Plate) que esta recubierta por una capa de fósforo fotoestimulable, durante un período de tiempo que osciló entre 1 y 5 horas. Posteriormente la superficie de la pantalla fue escaneada y se obtuvo una imagen que reveló la impresión causada por la radiación, permitiendo estimar el tamaño de los fragmentos de ADN que habían hibridado específicamente con la sonda.

\section{RLM-RACE (RNA Ligase Mediated Rapad Amplification of cADN Ends)}

El ARN total de C. albicans fue mapeado usando el kit "FirstChoice RLM-RACE" de Ambion. EI ARN se aisló de la cepa CAl4, creciendo en fase exponencial rompiendo las células en un "micro-dismembrator" (Braun, Melsungen). Después empleamos el método de extracción con trizol descrito en "Galar Fungal standared operating procedures". El proceso consiste en tratar la muestra de ARN total con un fosfato alcalino (CIP) que elimina los fosfatos $5^{\prime}$ libres de ARN degradado o ADN contaminante. A continuación la estructura cap del ARN de longitud completa es eliminada con la enzima pirofosfatasa ácido del Tabaco (TAP), quedando un grupo monofosfato libre en el extremo $5^{\prime}$. En este momento se liga a este grupo un adaptador (5'GCUGAUGGCGAUGAAUGAACACUGCGUUUGCUGGCUUUGAUGAAA 3') 
utilizando la ligasa de ARN T4. Seguidamente se procede a una trascripción reversa y el cADN obtenido es amplificado mediante PCR anidada. El producto de la segunda PCR puede ser clonado en pGEM-T y secuenciado.

\section{Ensayo de sensibilidad}

Las células se recogieron en fase exponencial, se prepararon suspensiones a una D.O $600_{\mathrm{nm}}$ de 0.4 y a partir de ellas se realizaron diluciones seriadas de 1/10. Se tomaron 5 $\mu$ l de cada una de las diluciones y se sembraron en placas de medio YEPD conteniendo diferentes concentraciones de los compuestos ensayadas Anfotericina $B$, Higromicina; Itraconazol, Flocunazol, $\mathrm{NaCl}$, Calcofluor white, Rojo congo, Cafeína, SDS, $\mathrm{KCl}, \mathrm{CaCl}_{2}$ y Zimoliasa.

\section{Ensayo de afinidad a Alcian blue}

El Alcian blue es un colorante catiónico no fluorescente que se une específicamente a las cadenas de fosfomanosas presentes en la superficie celular de las levaduras (Herrero et al., 2002). Este ensayo permite detectar defectos en la síntesis y secreción de las manoproteínas que forman parte de la pared celular. Las células recogidas en fase exponencial se lavaron 2 veces con $\mathrm{HCl} 0.02 \mathrm{~N}$ y a continuación se prepararon 1 $\mathrm{ml}$ a una D.O de 2.5 por duplicado de cada una de las cepas. Las muestras se centrifugaron resuspendiendo las células en $1.5 \mathrm{ml}$ de una solución de Alcian blue $0.005 \%$. Tras 10 minutos de incubación a temperatura ambiente, las muestras se centrifugaron 3 minutos a la máxima velocidad y seguidamente se midió la absorbancia a $600_{\mathrm{nm}}$ de los sobrenadantes. La concentración de Alcian blue de cada sobrenadante se determinó por comparación con una recta patrón que permitió relacionar la concentración de colorante con la absorbancia y cuyo coeficiente de correlación fue 1.00. A partir de esta concentración se estimó el porcentaje fijado por cada cepa considerando como $100 \%$ la concentración de Alcian blue del sobrenadante de una muestra tratada de la misma forma pero en la que no se incluyeron células.

\section{Ensayo de virulencia}

Una vez obtenidas las cepas, se prepararon cultivos de cada una de las cepas de $C$. albicans a ensayar en $100 \mathrm{ml}$ de YEPD a una D.O inicial de 0.03. Se incubaron hasta fase exponencial de crecimiento a $28^{\circ} \mathrm{C}$ con el fin de obtener células en forma de levadura. Las células se recogieron, se lavaron dos veces con PBS $1 \times$ y se resuspendieron a una concentración de $10^{8}$ y $5 \times 10^{8}$ células $/ \mathrm{ml}$. La concentración celular de la suspensión final se verificó en una cámara Thoma y por absorbancia a 
$600_{\mathrm{nm}}$. A partir de dicha suspensión se inocularon por vía intraperitoneal en 10 ratones CD1 con $10^{8}$ y $5 \times 10^{8}$ células de $C$. albicans cada uno. A partir del día de infección se realizó un seguimiento diario de la supervivencia de los ratones infectados que se prolongó hasta el día 30.

\section{Microscopía}

La observación de los microorganismos se realizó con un microscopio DMRXA (Leica) y un microscopio Leica DFC 350 FX acoplados a una cámara digital. La morfología de las colonias de C. albicans se examinó en un estereomicroscopio ZEISS Stemi SV-6.

\section{Aislamiento de ARN, síntesis de cADN e hibridación}

Las células se recogieron por centrifugación a 3000 rpm durante 5 minutos a temperatura ambiente. Los sobrenadantes se eliminaron mientras que el precipitado de células se resuspendió en el volumen residual de medio que quedó en el tubo de centrifugación. Seguidamente, las células se congelaron dejando caer gotas de aproximadamente $20 \mu \mathrm{l}$ en eppendorfs con nitrógeno líquido. Los tubos se mantuvieron en un recipiente con nitrógeno líquido para evitar la descongelación de las muestras, o bien se guardaron a $-80^{\circ} \mathrm{C}$ hasta su utilización. La lisis celular se llevó a cabo de forma mecánica dentro de una cámara de teflón en la que se introdujo, junto a la muestra, una esfera de tungsteno ( $7 \mathrm{~mm}$ de diámetro) que se sometió a agitación en un Micro-Dismembrator (Braun, Melsungen). Para el marcado con Cianina 3-dCTP o Cianina 5-dCTP (fluorocromos que absorben y emiten fluorescencia a diferentes longitudes de onda) se siguió el método de marcaje directo según el protocolo proporcionado por la casa comercial Eurogentec y en el que se introdujeron pequeñas modificaciones. De forma general puede decirse que se trata de una reacción de retro transcripción en la cual se incorporan los nucleótidos marcados durante la síntesis de las moléculas de cADN. En cada reacción se utilizaron $15 \mu \mathrm{g}$ de ARN total a los que se añadió $1 \mu \mathrm{l}$ del inhibidor de ribonucleasas ARNsin (Promega) y agua destilada tratada con DEPC hasta completar un volumen de $21 \mu$ l. Las soluciones de ARN se dejaron en un baño a $42^{\circ} \mathrm{C}$ mientras se mezclaron el resto de componentes de la reacción 5x First-strand buffer (Invitrogen) $(8 \mu \mathrm{l}), \quad$ C. albicans specific primer mix (Eurogentec) $(0.1 \mathrm{pmoles} / \mu \mathrm{l})(1 \mu \mathrm{l})$, Oligo dT (12-18) (Sigma) $(0.5 \mu \mathrm{g} / \mu \mathrm{l})(1 \mu \mathrm{l})$, Mezcla de dNTPs excepto dCTP (Sigma) (10 mM) $(3 \mu \mathrm{l})$, dCTP (Sigma) $(1 \mathrm{mM})(1 \mu \mathrm{l})$, Cianina 3-dCTP o Cianina 5-dCTP (1 mM).(CyDye TM Fluorescent nucleotides Cy3 o Cy5, Amersham) (1.5 $\mu \mathrm{l})$, DTT $(0.1 \mathrm{M})$ (Invitrogen) $(4 \mu \mathrm{l})$. La mezcla se realizó en 
eppendorfs cubiertos con papel de aluminio para evitar la exposición a la luz de los fluorocromos. La solución de ARN se añadió a la mezcla de reactivos y seguidamente se incubó 5 minutos a $65^{\circ} \mathrm{C}$ y 5 minutos a $42^{\circ} \mathrm{C}$. Se añadió $1 \mu \mathrm{l}$ de transcriptasa reversa (Invitrogen) y se incubó otra hora a $42^{\circ} \mathrm{C}$, añadiendo $1 \mu$ adicional de enzima transcurrido la mitad del tiempo de incubación. La reacción se detuvo añadiendo $5 \mu$ l de EDTA pH 8.0 y $2 \mu \mathrm{l}$ de $\mathrm{NaOH} 10 \mathrm{~N}$ e incubando la muestra 20 minutos a $65^{\circ} \mathrm{C}$. La purificación de las moléculas de cADN sintetizadas se llevó a cabo utilizando el kit de purificación de productos de PCR QIA-kit PCR purification kit (Quiagen) siguiendo el protocolo descrito por la casa comercial. Después de dos pasos de elución con $50 \mu \mathrm{l}$ de agua destilada cada uno, se cuantificó el cADN sintetizado, la cantidad de fluorocromo incorporado y la frecuencia de incorporación del mismo según las fórmulas que se indican a continuación. Dada la baja concentración de cADN que se obtiene, la absorbancia de la muestras se midió directamente, sin diluciones, utilizando ultramicrocubetas (Hellma). El volumen de muestra correspondiente a 65 pmoles de fluorocromo se concentró utilizando filtros microcon-30 (Amicon) hasta conseguir un volumen final de 5 a $10 \mu \mathrm{l}$. Para cada hibridación se mezclaron las muestras de cADN procedentes de cada una de las dos cepas a comparar (marcada una con Cy3 y otra con Cy5) y $5 \mu \mathrm{l}$ de ADN de esperma de salmón (Sigma-Aldrich) previamente desnaturalizado por calor. El cADN se desnaturalizó manteniendo la muestra 2 minutos a $95^{\circ} \mathrm{C}$ y enfriándola inmediatamente en hielo, seguidamente se añadió tampón de hibridación (Dig Easy Hybridization buffer, Roche) hasta un volumen final de $60 \mu \mathrm{l}$. La mezcla se depositó entonces sobre un cubreobjetos de plástico $(60 \times 24$ $\mathrm{mm}$, Sigma-Aldrich), el microarray se puso en contacto con la muestra por la cara que contenía el ADN y una vez que la muestra se extendió por aproximadamente la mitad del cubreobjetos se dió la vuelta al microarray y se colocó en una cámara de hibridación (Corning), que se sumergió en un baño a $42^{\circ} \mathrm{C}$. Después de 12 horas de hibridación, se retiró el cubreobjetos introduciendo el microarray en una cubeta con la primera solución de lavado (SDS $1 \%+$ SSC $2 \times$ ), se transfirió a un tubo falcon con la misma solución donde se mantuvo 5 minutos a temperatura ambiente con agitación ocasional y protegido de la luz. Para eliminar los restos de SDS, el microarray se lavó dos veces con $50 \mathrm{ml} \mathrm{SSC} 2 \times$, en el último de ellos se mantuvo 5 minutos con agitación ocasional. Por último, el microarray se secó mediante centrifugación a 1100 rpm durante 5 minutos a temperatura ambiente.

Se ha llevado a cabo la comparación de los perfiles de transcripción de dos mutantes de $C$. albicans con el de la cepa parental, CAI4, en cuatro condiciones de crecimiento: 
fase exponencial como levaduras, y a tres tiempos $(15,60$ y 180 minutos) de la inducción de la formación de hifas, en medio Lee a $37^{\circ} \mathrm{C}$. Para cada comparación mutante vs. CAl4 se han realizado cuatro hibridaciones correspondientes a dos réplicas biológicas, en los que el $A R N$ de la cepa mutante procede de cultivos independientes y dos hibridaciones para cada uno de ellos en los que el cADN se marcó de forma inversa (dye swap). El cADN de la cepa parental, CAI4, se sintetizó en cada condición a partir de una mezcla de ARN obtenido de diferentes cultivos de esta cepa provenientes de tres colonias. Considerando que para cada comparación se hibridaron 4 microarrays y que cada gen se encuentra depositado por duplicado en cada microarray, para la mayoría de los genes se analizaron 8 datos para cada condición (Fig. 4.2).

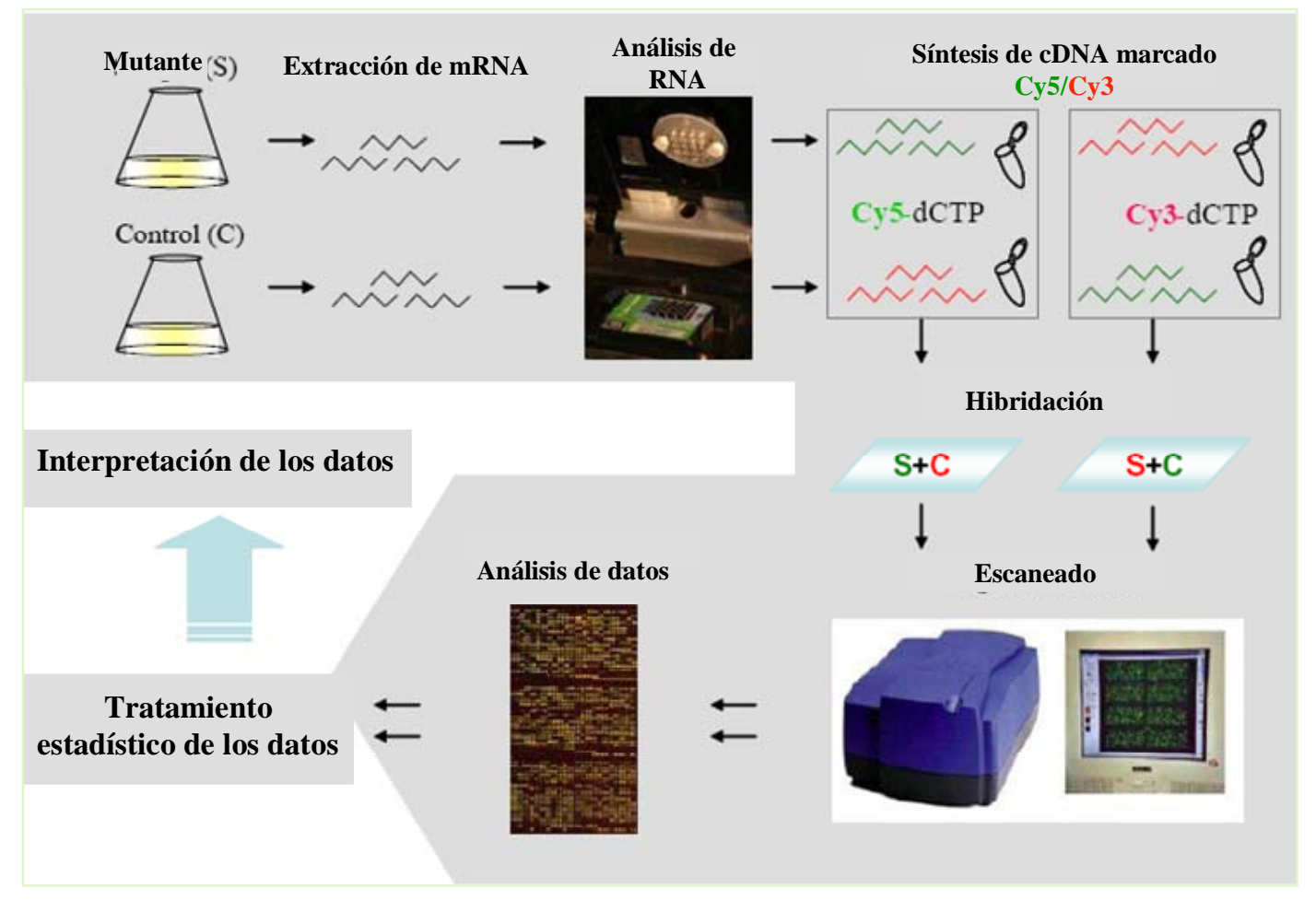

Figura 4.2 Esquema del protocolo seguido para el análisis comparativo del transcriptoma de dos cepas de C. albicans, mediante microarray de cADN de vidrio

\section{Análisis de los datos obtenidos}

La adquisición de las imágenes correspondientes a cada microarray se realizó en el Servicio de Genómica del Centro de Investigación del Cáncer (USAL-CSIC) con el escáner GenePix4000B y el programa asociado al mismo GenePix Pro 4.0 (Axon Instruments Inc.). Se llevaron a cabo varios escaneados, modificando los valores de fotomultiplicación (PMTs) asociados a cada fluorocromo, hasta obtener una imagen en la que las intensidades medias de ambos canales estuvieran equilibradas (ratio medio 
Cy5/Cy3 próximo a 1). El programa GeneSpring 5.0.3 (Agilent technologies, CA) fue utilizado a continuación para normalizar, integrar, realizar análisis de significación estadística y comparar los datos de diferentes microarrays. Los datos se sometieron a una normalización per spot y per chip dependiente de intensidad (Lowess) aplicada a cada uno de los bloques del microarray. Esta normalización permite comparar los datos de los diferentes genes, tanto si proceden del mismo como de diferentes microarrays, y al ser dependiente de intensidad, corrige las variaciones no significativas que dependen del nivel de expresión de cada gen. La detección de genes que se expresan de forma diferencial en el mutante en cada una de las condiciones analizadas se llevo a cabo según el test estadístico de comparación de grupos "Wilcoxon-Mann-Whitney" y la corrección para test múltiples "Benjamini and Hochberg FDR" con un valor de $\rho \leq 0.02$. De esta forma la probabilidad de error es de un $2 \%$ del total de los genes que pasan el test. El análisis de significación se realizó sólo sobre los genes que presentaban un factor de variación mínimo de 1.5 en al menos una hibridación, este punto de corte se introdujo para controlar la astringencia de la corrección "Benjamini and Hochberg FDR", que está influenciada directamente por el número de genes (Garcia-Sanchez et al., 2004). 


\section{Resultados}

\section{Determinación del sitio de inicio de la transcripción de los genes HTAT1 y HAT2} por método de RLM-RACE

Para identificar los sitios de inicio de la transcripción del gen HAT1 realizamos una RLM-RACE. El resultado se muestra en la Fig. 2.1A una banda de $\sim 450 \mathrm{pb}$, tres sitios putativos de inicio de la transcripción fueron identificadospara. Estos productos pudieron originarse a partir de tres sitios distintos de inicio de la transcripción o quizás las bandas más pequeñas pueden representar la terminación prematura de la reacción de transcripción reversa. Las tres posiciones de 21, 24 y 48 fueron consideradas como el sitio de inicio de la transcripción del gen HAT1 (Fig. 2.1B). El sitio de inicio de la transcripción del gen HAT2 aparentemente se encuentra en el interior de la fase codificante descrito por la aproximación "in silico". Experimentos de secuenciacion de la proteina purificada o análisis de la ORF en otras cepas de Candida son necesario para elucidar este punto (Fig. 2.1C).

\section{Interrupción del gen HAT1 en la cepa RDH4 (hat2A)}

Nuestro grupo, dentro del proyecto de investigación en el que se incluye el presente trabajo, está llevando a cabo el análisis sistemático de la implicación de diferentes histonas acetiltransferasas e histonas desacetilasas en la regulación de la transición levadura-hifa y de otros procesos morfogenéticos. El primer objetivo ha sido la interrupción de los dos alelos del gen HAT1 en la cepa RDH4 (con el gen HAT2 delecionada que había sido obtenida previamente en nuestro laboratorio). Ello se llevó a cabo siguiendo la estrategia del "Ura-blaster" (Fonzi \& Irwin, 1993). En la Fig. 2.3B se muestra la comprobación del genotipo de las cepas generadas mediante Southern. El ADN genómico fue digerido con Ssp I y como sonda se utilizó la secuencia de 459 pb correspondiente a la región $3^{\prime}$ no codificante (promotor) del gen HAT1. Tal y como puede apreciarse en la Fig. 2.3B, las cepas CAI4, RDH4 y heterocigotas para la deleción del gen presentan la banda de $0.64 \mathrm{~kb}$ correspondiente al alelo silvestre de HAT1. Esta banda no aparece en las cepas homocigotas para la deleción de HAT1 (MAH3 y MAH4) y en su lugar se detectan otras bandas cuyo tamaño se ajusta a la secuencia del cassette de deleción y de las secuencias flanqueantes.

\section{Efecto de la doble deleción hat1 $\Delta$, hda2 $\Delta$ en el crecimiento exponencial}

Mediante el seguimiento de la D.O alcanzada a lo largo del tiempo por cultivos de las diferentes cepas, se detectó un retraso en el crecimiento del doble mutante hat $1 \Delta$, 
hat2 $\Delta$ en medio YEPD respecto a la cepa parental CAI4. Se observó que en fase exponencial el tiempo de generación de las cepas MAH4 era mayor al de la cepa parental CAI4. Estimamos que en este medio la cepa MAH4 iniciaba la fase exponencial tras aproximadamente 16 horas de crecimiento (Fig. 2.4).

\section{Efecto de la doble deleción hat1 $\Delta$, hat2 $\Delta$ en la filamentación en medios sólidos}

Hemos analizado el análisis del fenotipo del doble mutante hat1 $\Delta$, hat2 $\Delta$ (cepa MAH4), de los dos mutantes simples y de la cepa CAI4 durante crecimiento de forma levadura e hifa. En la Fig. 2.6 se puede apreciar claramente que el doble mutante presenta una morfología hiperfilamentosa como el mutante simple hat1 $\Delta$ en los medios inductores de filamentación. El aspecto de las colonias en el medio sólido YEPD se muestra en la Fig. 2.5A y presenta mayor rugosidad.

\section{Efecto de la doble deleción hat1 $\Delta$, hat2 $\Delta$ en la transición levadura-hifa en medio líquilo}

Después de inducir la transición dimórfica en medio líquido Lee con suero al $4 \%$ a $37^{\circ} \mathrm{C}$ se observó que el mutante doble hat $1 \Delta$, hat $2 \Delta$ es incapaz de formar hifas, igual el mutante simple hat $1 \Delta$. Ambas cepas forman en lugar de hifas, pseudohifas. El doble mutante se comporta así, de diferente forma que el mutante simple hat $2 \Delta$, ya que en medios líquidos si es incapaz de formar hifas (Fig. 2.7). En la inducción en medio Lee a $37^{\circ} \mathrm{C}$ observamos el mismo efecto (datos no mostrados).

\section{Efecto de las deleción de los genes HAT1 y HAT2 en la formación de clamidosporas}

A continuación se analizó el efecto de la deleción de los genes HAT1 y HAT2 en la formación de clamidosporas. Las cepas se incubaron en medio cornmeal agar bajo condiciones microaerófilas y a temperatura $25^{\circ} \mathrm{C}$, hemos observado que la cepa doble mutante hat $1 \Delta$, hat $2 \Delta$ es capaz de formar clamidosporas al igual que ambos mutantes simples (Fig. 2.8).

\section{Ensayo de sensibilidad}

Hemos analizado la sensibilidad de estas cepas a los siguientes compuestos: Cafeína, Calcofluor white, $\mathrm{NaCl}$, SDS, Rojo congo, Fluoconazol, Itraconazol, Higromicina y Anfotericina B. Como se puede observar en la Fig. 2.9 se encontraron diferencias de sensibilidad a las concentraciones señaladas de Calcofluor white, $\mathrm{NaCl}$, Cafeína y Rojo congo entre el doble mutante y el mutante hat $1 \Delta$ con respecto a la cepa CAI4. En 
S. cerevisiae la hipersensibilidad a Cafeína se ha relacionado con defectos en los mecanismos reguladores de la formación de la pared celular. Todos estos procesos parecen estar alterados en la cepa doble delecionada.

Para el ensayo de sensibilidad a Zimoliasa, tomamos como referencia estudios previos realizados en nuestro laboratorio (Herrero et al., 1999b). El resultado obtenido se muestra en la Fig. 2.10. Como puede observarse, la caída de la densidad óptica a largo del tiempo es más rápido en el doble mutante y mutante sencillo hat1 $\Delta$. Este resultado demuestra que el doble mutante hat $1 \Delta$, hat $2 \Delta$ y el mutante hat $1 \Delta$ son más sensibles a la Zimoliasa. La mayor sensibilidad del mutante hat1 $\Delta$ y hat $1 \Delta$, hat2 $\Delta$, que la cepa CAl4, frente a la $\beta-1,3$ glucanasa (Zimoliasa) sugiere una alteración de la pared celular de estos mutantes.

\section{Ensayo de afinidad a Alcian blue}

Uno de los ensayos utilizados para determinar la existencia de posibles alteraciones en pared celular, es el ensayo de "Alcian blue". En la Fig. 2.11 se muestran los valores del colorante fijado por la cepa CAI4 y los mutantes de C. albicans. Si tomamos como $100 \%$ la cantidad de colorante fijado por las células de la cepa CAl4, las células del doble mutante fijan un $92.36 \%$. El porcentaje de colorante fijado por las cepas RDH4 y RGL4 es de un $118,94 \%$ y $48,14 \%$ respectivamente. En C. albicans, la capacidad de adhesión depende de su pared celular (Sundstrom, 1999). Estudios han revelado que las manoproteínas una vez glicosiladas interaccionan directamente con los células del hospedador es decir, el proceso de glicosilación es un factor importante para la virulencia (Timpel et al., 2000). Durante el proceso de transición levadura-hifa existe un rápida remodelación y expansión de la pared celular; por esta razón, determinadas mutaciones en los genes que intervienen en la formación de la pared celular dan lugar a cepas con defectos en la filamentación (Herrero et al., 2002; Timpel et al., 2000). Además, los genes específicos para la formación hifas en C. albicans codifican proteínas de secreción de la pared celular. Así que, es de esperar que defectos en cualquiera de las rutas de señalización que regulan el proceso de transición levadurahifa, se vean reflejados en la alteración de algunos componentes de la pared celular.

\section{Virulencia}

El ensayo de virulencia se llevó a cabo con las cepas: RGLR6 obtenida tras la reconstrucción de uno de los locus URA3 de la cepa RGL4 (hat1A), RDHR9 obtenida de la cepa RDH4 (hat2 $\Delta$ ) y MAHR1 obtenida de la cepa MAH4 (hat1 $\Delta$, hat2 $\Delta$ ). Se inyectó por vía intra-peritoneal a cada uno de los ratones una suspensión de $5 \times 10^{8}$ 
células en $200 \mu \mathrm{l}$ de PBS 1×. A partir del día de infección se realizó un seguimiento diario de la supervivencia de los ratones infectados que se prolongó hasta el día 30. La curva de supervivencia se muestra que efectivamente no hay diferencias significativas entre los resultados obtenidos para el mutante simple hat $2 \Delta$ y la cepa CAl4. La deleción del gen HAT2 no parece causar, por tanto, alteraciones en la virulencia de $C$. albicans, al menos bajo las condiciónes ensayadas.

Tal y como se muestra en la grafica (Fig. 2.12), el resultados obtenidos con las cepas RGLR6 y MAHR1 no es similar al obtenido con la cepa control CAMR. En los mutantes simple hat $1 \Delta$ y doble hat $1 \Delta$, hat $2 \Delta$ se muestró una reduccion $(40 \%)$ y $(30 \%)$ en la virulencia respectivamente.

\section{Localización subcelular de la proteína CaHat1p y CaHat1p}

Para la fusión de ambas proteínas, la ORF de HAT1 y HAT2 se amplificaron desde el codón de inicio hasta el codón previo al de terminación mediante PCR usando como molde ADN de la cepa CAI4 y como oligonucleótidos HAT1-GFPF y HAT1-GFPR (la ORF del gen HAT1) y HAT2-GFPU y HAT2-GFPR (la ORF del gen HAT2). El fragmento de 1260 pb (gen HAT1) y 1473 pb (gen HAT2) se clonó en el plásmido pAG1 (Gonzalez-Novo et al., 2004). Entre los transformantes obtenidos se seleccionó uno de aquellos en los que el gen HAT1 y HAT1 quedó insertado en el mismo sentido que el gen GFP3. Se obtuvo así los plásmidos pAHR1 y pZRG1 en el que las secuencias HAT1-GFP3 y HAT2-GFP3 constituyen una única fase de lectura abierta. El vector pAHR1 y pZRG1 se utilizó para transformar células de las cepas CAI4, RGL4 (hat1 $\Delta$ ), RDH4 (hat2 $\Delta$ ) y MAH4 (hat1 $\Delta$, hat2 $\Delta$ ). Se seleccionaron clones de cada una de las transformaciones y se dejaron crecer en YNB a $28^{\circ} \mathrm{C}$. Los cultivos se refrescaron mediante una dilución $1 / 5$ del cultivo en medio YNB y se incubaron durante 5 horas a $28^{\circ} \mathrm{C}$. Se tomó una muestra de cada uno de los transformantes y se observó en un microscopio óptico DRMX (Leica) con un fuente de UV Leica ebq 100, utilizando los filtros adecuados para la visualización de la fluorescencia de la GFP y del colorante Hoechst. Se observó que las proteínas Hat1p y Hat2p se localizaban en un punto discreto dentro de cada célula. La tinción con Hoechst, colorante fluorescente que se une al ADN, permitió determinar que se trataba del núcleo (Fig. 2.13 y 2.14). En la segunda parte de este trabajo, hemos transformado el vector pAHR1 para transformar células de la cepa RDH4 (hat2 $\Delta$ ). En los clones analizados se observó que la proteína Hat1p se localizaba en el núcleo (Fig. 2.15A). Por ultimo, hemos transformado con el vector pZRG1 la cepa RGL4 (hat1 $\Delta$ ). En los clones analizados se observó la proteína Hat2p, no se localizaba en el núcleo (Fig. 2.15B). Ello indica que la localización nuclear de Hat2p depende de Hat1p. 
Hemos realizado un estudio por el sistema de doble híbrido para identificar sí proteína Hat1p tiene interacción directa con Hat2p. Para este rastreo se construyó un plásmido (Tabla 2.1) que contenía la secuencia que codifica Hat1p y Hat2p. Se confirmó que la interacción entre las proteínas Hat1 y Hat2 no existe en el ensayo de doble híbrido. Lo que sugiere que debe existir una proteina que haga de puente similar a la Hif1 de $S$. cerevisiae aun que esto no le ha detectado mediante analisis blott en ninguna otra levadura.

\section{Análisis del transcriptoma de mutante hat $2 \Delta$ durante crecimiento en forma de} levadura

A partir de células recogidas en fase de crecimiento exponencial como levaduras, se detectaron 109 genes cuya expresión se alteraba significativamente en el mutante hat2 $\Delta$. Se detectaron dos genes comunes (HHF1 y STF2) entre hat1 $\Delta$ y hat2 $\Delta$. Dentro del grupo de genes sobreexpresados se detectaron genes implicando en las siguientes categorías: respiración (ATP6, COX3A, COX3B, NAD2, NAD5, NAD6 y STF2) y ensamblaje de histonas (HHF1, HTA1 y NHP6A). No hemos detectado genes comunes relacionados con las mismas funciones en los mutantes hat1 $\Delta$ y doble hat $1 \Delta$, hat2 $\Delta$ durante el crecimiento en forma de levadura. Además se detectaron genes subexpresados relacionados con glicólisis (CDC19, FBA1, PDB1, PFK2 y PYC2), ciclo TCA (ACO1, ACO2, CIT1, GDH3, MDH1-1 y MIS11), genes que codifican para proteínas ribosomales (RPL8B, RPL13, RPL16A, RPL81, RPP0, RPS4A, RPS8A, RPS15, RPS23A y YST1), genes que codifican proteínas de pared celular (PGA45, PGA59, PGA62, TOS1 y YWP1), genes de respuesta a estrés (SOD1, SOD2, SSB1 y HSP90) y factores de transcripción (EFG1, NRG1, RGT1 y SKO1). CaSKO1 codifica un factor transcripcional cuyo homólogo en $S$. cerevisiae tiene un efecto represor de la actividad de la ruta PKA-cAMP y que es capaz de unirse al promotor de ScSOK2 (homólogo de CaEFG1) (Nehlin et al., 1992; Proft et al., 2005). En conjunto estos datos demuestran que el perfil transcripcional del mutante hat $2 \Delta$ es diferente del de los mutantes hat $1 \Delta \mathrm{y}$ doble hat $1 \Delta$, hat $2 \Delta$ lo que sugiere que Hat2p tiene funciones celulares especificas.

\section{Análisis del perfil de la transcripción de hat2 $\Delta$ durante la transición levadura-hifa}

El análisis del perfil transcripcional de la cepa hat2 $\Delta$ en comparación con el de la cepa parental indicó que durante la transición levadura-hifa, la deleción del HAT2 altera la 
expresión de al menos 429 genes, con un factor de variación mutante vs cepa control $\geq 1.5$. De éstos, 196 genes son sobreexprsados y 233 genes subexpresados. 2 genes son comunes a los tres tiempos (sobreexpresados), 54 genes entre los 15 y 60 min (35 sobre- 19 sub-expresados) y 11 genes entre los 15 y 180 min (9 sobre- y 2 subexpresados). 156 genes (85 sobre- y 71 sub-expresados), 167 genes (40 genes sobrey 127 sub-expresados) y 39 genes ( 25 sobre- y 14 sub-expresados) solo varian en un tiempo de inducción (15, 60 y $180 \mathrm{~min}$ respectivamenete). Considerando datos publicados por diversos autores sobre la modulacion de la expresión genica durante la transición levadura-hifa, se trató de determinar que genes implicados en diversos categoria se encontraban alterados en el mutante hat $2 \Delta$ durante la transición levadurahifa. Se ha detectado la sobreexpresion de 17 genes relacionados con metabolismo de carbohidratos (principalmente genes relacionados con la ruta de glicolisis) (e.g $A D H 1$, $A D H 5, E N O 1, F A B 1$ y PCK1) durante 15 min, 9 genes de pared celular (e.g BMT5, BMT9, CHS2, GSL1, HWP1, PGA45 y PGA54), 5 genes de transporte de azúcares (HGT1, HGT7, HGT8, HGT9 y SHA3), 3 genes de la familia de la telomerasa (TLO3, TLO4 y TLO12) y 5 genes de proteínas ribosimales (RPL4B, RPL5, RPL33, RPL42 y RPS6A) (Tabla 2.4). La regulación de la expresión de genes de glicolisis se ha asociado en diferentes trabajos a la transición dimórfica, observándose que en el inicio de la transición levadura-hifa la expresión de genes que codifican enzimas de la ruta de glicolisis se reduce drásticamente y a continuación aumenta progresivamente, llegando a ser en algunos genes mayor en hifas que en levaduras. Sin embargo, su relación con morfogénesis no ha sido esclarecida, incluso se ha sugerido que se debe a las variaciones fisiológicas subyacentes al cambio en las condiciones de crecimiento y no al proceso morfogenético en sí (Rashki, Tesis doctoral 2009; Swoboda et al., 1994). Ademas, observándose que en el inicio de la transición levadura-hifa (15 min) la expresión de un número de los genes que codifican enzimas de la ruta de glicolisis se aumente en el mutante gcn5 $\Delta$ (Degano, Tesis doctoral en proceso). Dentro del grupo de los genes subexpresados, se ha observado 7 genes relacionados con respiración (COX1, COX3A, COX3B, COX6, COX15, NAD2 y NAD6), 9 genes de pared celular (ALS4, ECM31, GCS1, HYR1, HYR3, PHR2, PGA6, PGA63 y TOS1), 12 genes relacionados con proteínas ribosomales (MRPL19, MRPL38, RPL8B, RPL10, RPL11, RPL14B, RPL16A, RPL20B, RPL82, RPS4A, RPS15 y RPS16), 8 genes que codifican para factores de transcripción (CTA4, CTA8, CPH1, HAC1, SPT20, TBP1, ZCF20 y ZPR1) y por último 4 genes relacionados con biosíntesis de ergosterol (ERG4, ERG7, ERG9 y ERG26) (Tabla 2.4). HAC1 codifica el homólogo en C. albicans del activador transcripcional ScHac1p de S. cerevisiae. ScHac1p es capaz de interaccionar con el 
motivo UPRE, asociado a proteínas de respuesta a choque térmico implicadas en el plegamiento de proteínas, y con el motivo de respuesta a cAMP (secuencia CRE) (Mori et al., 1996; Nojima et al., 1994). El cAMP actúa como segundo mensajero en una de las principales rutas de transducción de señales que controlan la filamentación en C. albicans. La expresión de CaHAC1 se induce durante la miceliación a $37^{\circ} \mathrm{C}$ en la cepa silvestre de forma coordinada con otros reguladores morfogenéticos, por lo que la activación de este gen en $C$. albicans podría constituir una respuesta al aumento de la temperatura de incubación. Hasta el momento no se ha analizado su posible asociación con la regulación de la transición dimórfica. El estudio de un mutante nulo Cahac1 podría resultar interesante ya que permitiría determinar si la regulación dependiente de Hac1p juega un papel relevante en la transición levadura-hifa inducida por temperatura.

\section{Análisis del transcriptoma del doble mutante hat1 $\Delta$, hat2 $\Delta$ durante la fase de} levadura

Los perfiles de transcripción se utilizaron para examinar más a fondo el papel de ambas acetiltransferasas en $C$. albicans. El transcriptoma del mutante doble hat1A, hat $2 \Delta$ se compararó con la cepa CAI4 durante la crecimiento levadura y durante la transición levadura-hifa. Anteriormente en nuestro laboratorio se han llevado a cabo experimentos, en los que se ha analizado los transcriptomas del mutante simple hat1 $\Delta$. Tal y como se ha señalado anteriormente, en las 4 condiciones examinadas, crecimiento exponencial como levaduras (en YNB a $28^{\circ} \mathrm{C}$ ) y a tres tiempos $(15,60$ y $180 \mathrm{~min}$ ) durante la transición dimórfica en medio líquido Lee a $37^{\circ} \mathrm{C}$, el mutante doble hat $1 \Delta$, hat $2 \Delta$ muestra diferencias morfológicas respecto a la cepa parental. A partir de células recogidas en fase exponencial de crecimiento como levaduras, se detectó que la expresión de 219 genes cuya expresión se encontraba alterada por la deleción de HAT1 y HAT2 en el crecimiento en forma de levadura. El número de genes que presentan expresión diferencial en el mutante doble hat1 $\Delta$, hat2 $\Delta$ es mayor que el detectado en los mutantes simples hat $1 \Delta$ y hat $2 \Delta$. De los genes expresados en el doble mutante con respecto al silvestre durante crecimiento de forma levadura, 37 son de función desconocida; el resto tienen una función conocida o deducida por homología a partir de la secuencia correspondiente de Saccharomyces cerevisiae. El gen HSP12 presenta un incremento significativo en la expresión en el doble mutante hat1 $\Delta$, hat2 $\Delta$ con respecto al silvestre (con una relación mutante/silvestre de 5,06 ) dicho gen se encuentra reprimido en condiciones de inducción de filamentación (Lane et al., 2001; Nantel et al., 2002). HSP12 es una proteína de choque térmico implicada 
en la organización de la pared celular cuya transcripción se induce en una gran variedad de condiciones de estrés que incluyen: estrés osmótico, estrés oxidativo, estrés térmico, presencia de etanol, limitación de nutrientes por crecimiento en fuentes de carbono no fermentables así como la presencia agentes que afectan a la integridad de la pared celular (Karreman \& Lindsey, 2005). Además se detectó la presencia de los genes HSP31, HSP70, HSP78, HSP104 que codifican para proteínas de choque térmico. Otros genes sobreexpresados en la cepa hat $1 \Delta$, hat $2 \Delta$ durante el crecimiento en forma de levadura es el gen que codifica la histona H4, HHF22, y dos genes que codifican para la histona H3, HHT1 y HHT21. Ello parece indicar la existencia de mecanismos de activación de genes por represión de los genes H4 y H3. Cabe destacar que dentro de la subcategoría de regulación positiva de la transcripción aparecen sobreexpresadas 5 genes (TLO1, TLO3, TLO11, TLO12 y TLO16) pertenecientes a una misma familia de genes telomericos. Se trata del grupo de probables activadores transcripcionales de C. albicans denominado CTA2. Las características de esta familia de genes apenas se han estudiado hasta el momento. Además se encuentra el grupo de subunidades de proteosoma (PRE3, PRE4, PRE6, PRE7, PRE9, PUP2, RPN4 y RPN12).

\section{Análisis del transcriptoma del doble mutante hat $1 \Delta$, hat $2 \Delta$ durante la transición} levadura-hifa

Hemos analizado el perfil transcripcional de la cepa MAH4 para profundizar en el estudio de la regulación de la expresión en esta cepa, prestando especial interés a los genes implicados en la formación de hifas. Para ello, se realizaron comparaciones entre los niveles de expresión genética de la transición levadura-hifa a los 15, 60 y 180 minutos. El resultado que obtuvimos fue que 787 genes alteraban significativamente su expresión, en al menos uno de los tres tiempos. De estos genes, 417 están subexpresados y 370 están sobreexpresados respecto a la cepa CAl4. 16 genes son comunes a los tres tiempos (6 genes sobr- y 10 genes subexpresados), 193 genes entre los 15 y 60 min (62 sobre- 131 sub-expresados) y16 genes entre los 60 y 180 min (10 sobre- y 6 sub-expresados) (Tabla 2.6). 272 genes (189 sobre- y 83 subexpresados), 265 genes (97 genes sobre- y 168 sub-expresados) y 29 genes (10 sobre- y 19 sub-expresados) solo varian en un tiempo de inducción (15, 60 y 180 min respectivamenete). Considerando datos publicados por diversos autores sobre la modulacion de la expresión genica durante la transición levadura-hifa, se trató de determinar que genes implicados en diversos categoria se encontraban alterados en el mutante doble hat $1 \Delta$, hat2 $\Delta$ durante la transición levadura-hifa. 
Se detecta un número elevado tanto de genes sobreexpresados como subexpresados. Se encuentran genes cuya expresión diferencial se manifiesta principalmente a la $1 \mathrm{~h}$, cuando las hifas/pseudohifas están formados. De acuerdo a las diferencias morfológicas entre ambas cepas, en este grupo se detecta la subexpresión de genes que codifican proteínas principalmente asociadas a la pared celular [CaAls1p, CaAls $3 p$, CaAls $4 p$ (15 y $60 \mathrm{~min}$ ), CaAls $2 p$ (15 min) y CaAls5p (60 min)] y genes implicados en transporte de azúcares HGT6, HGT7 y HGT8 (Fan et al., 2002), genes implicados en la secreción y procesamiento de proteínas SEC23 y SEC26 (15 min), SEC9, SEC11, SEC16, SEC24 y SEC61 (60 min), PMT1, PMT2 y PMT4 (15 y 60 min) (Maglott et al., 2007; Prill et al., 2005; Timpel et al., 1998) durante 15 y 60 min de transition levadura hifa. Probablemente este efecto se debe al mayor requerimiento de transporte de material hacia el ápice de las hifas, lugar donde se concentra la síntesis de pared celular durante el crecimiento polarizado (Mondesert et al., 1997; Nantel et al., 2002; Whiteway \& Oberholzer, 2004). También se han detectado posibles genes implicados en la secreción y procesamiento de proteínas (SEC16, SEC23, SEC26, PMT1, PMT2 y PMT4) sobreexpresados en el mutante odc1 durante 15 y 60 min de la transition levadura hifa (Rashki, Tesis doctoral 2009). La regulación de la expresión de genes de procesamiento de proteínas se ha asociado en diferentes trabajos (Cantero et al., 2007). Entre los genes sobreexpresados, se observó 37 genes (15 min), 3 genes (60 min), 9 genes (15 y $60 \mathrm{~min}), 1$ gene (60 y $180 \mathrm{~min}$ ) y 2 genes $(15,60$ y $180 \mathrm{~min}$ ) relacionados con proteínas ribosomales. Se ha reportado que 63 genes son subexpresados durante el crecimiento en forma de levadura en el mutante simple gcn5 $\Delta$ (Degano, Tesis doctoral en proceso). Además hemos observado que 12 genes que codifican para la misma categoría son subexpresados y 5 genes sobreexpresados en hat $2 \Delta$ durante transición levadura-hifa, indicando la diferencia entre mutante doble y simple hat $2 \Delta$. Se ha demostrado que la deletion de los genes, GCN5 y HDA1, conlleva la subexpresión un numero de genes que codifican proteínas ribosomales (Rashki, Tesis doctoral 2009). Hemos encontrado que 4 genes relacionados con transporte de drogas (CDR1, CDR2, CDR4 y QDR1) y 14 genes implicados en factores de transcripción están subexpresados en el mutante doble. Otros grupos de genes subexpresados son: genes relacionados con pared celular y glicólisis. Además, se detectó genes sobreexpresados en categorías como respuesta a estrés y pared celular. 
La interrupción de los dos alelos del gen SIN3 en la cepa RDG4 (con el gen GCN5 delecionado que había sido previamente construida en nuestro laboratorio) se llevó a cabo siguiendo la estrategia del "Ura-blaster". En la Fig. $3.1 \mathrm{~B}$ se muestra la comprobación del genotipo de las cepas generadas mediante Southern. EI ADN genómico fue digerido con $\mathrm{Ndel}$ y como sonda se utilizó la secuencia de 588 pb correspondiente a la región $3^{\prime}$ no codificante del gen SIN3. Como puede apreciarse en la Fig. 3.1, las cepas CAI4, RDG4 y los heterocigotos para la deleción del gen presentan la banda de $3.6 \mathrm{~kb}$ correspondiente al alelo silvestre de SIN3. Esta banda no aparece en las cepas homocigotas para la deleción de SIN3 (ZAH4) y en su lugar se detectan otras bandas cuyo tamaño se ajusta a la secuencia del cassette de deleción y de las secuencias flanqueantes.

\section{Efecto de la doble deleción gcn5 $\sin 3 \Delta$ en el crecimiento exponencial}

Mediante el seguimiento de la D.O alcanzada a lo largo del tiempo por los cultivos de las diferentes cepas, se detectó un retraso en el crecimiento del doble mutante gcn5 $\Delta$, $\sin 3 \Delta$ en comparación con la cepa CAI4 en medio YEPD. Las curvas de crecimiento obtenidas para los mutantes ZAH4 $(g c n 5 \Delta, \sin 3 \Delta)$ y SFC4 $(\sin 3 \Delta)$ fueron muy similares entre sí y, a su vez, diferentes a las cepas CAI4 y RDG4 (gcn5 $\Delta$ ). Estimamos que en este medio las cepas ZAH4 iniciaban la fase exponencial tras aproximadamente 18 horas de crecimiento (Fig. 3.2). Las células de la cepa doble delecionada son alargadas, lo mismo que hemos visto en las células de la cepa delecionada para el gen SIN3, y también gromada, lo que ha visto en las células de la cepa delecionada para el gen GCN5. La morfológia presenta defectos que son muy claros en ausencia de ambos genes (Fig. 3.3B).

\section{Efecto de la doble deleción gcn5 $\Delta, \sin 3 \Delta$ en la fillamentación en medios sólidos}

El aspecto de las colonias de las cepas CAI4, RDG4, SFC4 y ZAH4 en los medios sólidos estudiados se muestra en la Fig. 3.4. El medio Spider es un inductor de miceliación en C. albicans (Liu et al., 1994). En este medio las colonias de la cepa control (CAI4) presentan filamentos que emergen alrededor de las mismas. Los mutantes simples y el doble mutante presentan defectos de filamentación. También se ha analizado el comportamiento de las células de diferentes cepas creciendo embebidas dentro de una matriz de agar y en estas condiciones la cepa CAI4 tiene lugar una rápida producción de hifas (Brown et al., 1999), lo que podría jugar un papel 
importante durante la infección del tejido del hospedador (Giusani et al., 2002). Los resultados demuestran que el doble mutante $g \operatorname{cn} 5 \Delta, \sin 3 \Delta$ al igual que mutante simple gcn5 $\Delta$ es incapaz de producir hifas en esta condición de inducción. Siguiendo con la dinámica del estudio de la morfología de las colonias del doble mutante $g c n 5 \Delta, \sin 3 \Delta$ de C. albicans, se utilizó el medio SLADH. Este medio tiene bajo contenido en fuente de nitrógeno e induce crecimiento invasivo y pseudofilamentoso en $S$. cerevisiae y crecimiento en forma de hifas verdaderas en C. albicans (Csank et al., 1998; Ernst, 2000). Las colonias del doble mutante forman colonias de aspecto rugoso y son incapaces de filamentar. En el medio Lee con NAcGlc, las colonias del doble mutante forman filamentos que penetran el agar. En la Fig. 3.4 se observa como los filamentos de la colonia del doble mutante están por debajo del agar y los de la cepa CAI4 están en la superficie. Por ultimo, en el medio Lee con suero al $4 \%$, las colonias del doble mutante son incapaces de formar filamentos como ambos mutantes simples.

\section{Efecto de la doble deleción gcn5 $\sin 3 \Delta$ en la transición dimórfica en medio} líquido

La inducción de micelio se llevó a cabo en las cepas CAl4 y en el doble mutante gcn $5 \Delta$, sin3 $\Delta$ de $C$. albicans. Para ello, después de un reposo metabólico a $4^{\circ} \mathrm{C}$, las células se crecieron en medio Lee suplementado con uridina y suero al $4 \%$ a $37^{\circ} \mathrm{C}$. La cepa silvestre forma hifas verdaderas a partir de un tubo germinativo que ya puede apreciarse claramente a los $60 \mathrm{~min}$ de inducción. En la cepa doble mutante gcn5 $\Delta$, $\sin 3 \Delta$, por el contrario, pueden visualizar se pseudohifas y agregados celulares (Fig 3.5) al igual que en los mutantes simples.

\section{Efecto de la deleción de los genes GCN5 y SIN3 en la formación de} clamidosporas

La inducción de la formación de clamidosporas se llevó a cabo de forma análoga a la

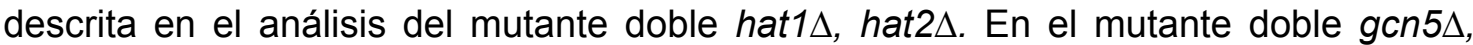
$\sin 3 \Delta$, así como en los mutantes simples no se detectaron clamidosporas, ni siquiera después de un periodo de incubación prolongado (14 días) (Fig. 3.6). Sonneborn et al (1999a) describieron para el mutante efg1 un fenotipo muy similar bajo las mismas condiciones de inducción de la formación de clamidosporas. Estas observaciones apoyan la hipótesis de la existencia de interacción entre Efg1p y $\operatorname{Sin} 3 p$ o Gcn5p expuesta en trabajos previos de nuestro grupo de investigación (Monterola, 2002; Tebarth et al., 2003). Como se ha indicado anteriormente, el requerimiento de los procesos de remodelación de la cromatina sobre la formación de clamidosporas se ha 
hecho evidente por la observación de que una de las proteínas implicada en su desarrollo es Calsw2p (Nobile et al., 2003), cuyo homólogo de S. cerevisiae forma parte de un complejo remodelador de la cromatina, que actúa en paralelo con el complejo Rpd3p-Sin3p de S. cerevisiae (Fazzio et al., 2001). Otro gen cuya alteración causa defectos en la diferenciación de clamidosporas es $\mathrm{CaSCH}$, que codifica una MAP quinasa que interviene en la regulación de diferentes procesos, entre los que se encuentra el almacenamiento de glucógeno, lo que está en concordancia con la función de las clamidosporas como estructuras de reserva (Nobile et al., 2003). El mutante $\sin 3 \Delta$ en $S$. cerevisiae presenta menor acumulación de glucógeno que la cepa silvestre (Wilson et al., 2002), sería interesante por tanto determinar si la función de CaSin $3 p$ en el metabolismo de este polisacárido se encuentra conservada en $C$. albicans, de forma que pudieran relacionarse los defectos de acumulación de glucógeno con la incapacidad de formar clamidosporas. Se ha sugerido que la existencia en el genoma de $C$. albicans de numerosos genes sin homólogo conocido en otros organismos podría estar relacionada con el desarrollo de este proceso casi específico de $C$. albicans. Son necesarios por tanto nuevos trabajos sobre los mecanismos reguladores que desencadenan la formación de clamidosporas.

Efecto de la deleción de los genes GCN5 y SIN3 en la estructura de la pared celular

En el ensayo de sensibilidad a Zimoliasa se muestra en la Fig. 3.8. Como puede observarse, el doble mutante muestra un fenotipo igual al del mutante sencillo $\sin 3 \Delta$. Por último, decidimos analizar la sensibilidad a otros agentes que afectan a la pared celular: Cafeína, Calcofluor white, $\mathrm{NaCl}$, SDS y Rojo congo. El doble mutante gcn5 $\sin 3 \Delta$ y el mutante sencillo gcn $5 \Delta$ son sensibles a Higromicina a una concentración de 100 y $300 \mu \mathrm{g} / \mathrm{ml}$. Como se puede observar en la Fig. 3.7, también son más sensibles a Cafeína, Itraconazol, Fluconazol, $\mathrm{NaCl}, \mathrm{KCl}, \mathrm{CaCL}_{2}$ y Rojo congo. En la Fig. 3.9 se muestran los valores del colorante fijado por la cepa CAI4, RDG4, SFC4 y ZAH4 de C. albicans. Si tomamos como $100 \%$ la cantidad de colorante fijado por las células de la cepa CAI4, las células del doble mutante fijan un 73,44 \%. El porcentaje de colorante fijado por las cepas SFC4 y RDG4 es de un 40,44\% y 161,83\% respectivamente. Además, podemos concluir que en términos absolutos el mutante simple $\sin 3 \Delta$ y la cepa doble delecionada fijan menos colorante que el mutante gcn5 y la cepa CAI4. Estas diferencias se pueden atribuir a que el doble mutante y mutante simple $\sin 3 \Delta$, presentan una menor cantidad de fosfato en las manoproteínas presentes en la pared celular. 


\section{Virulencia}

Hemos usado la cepa EVA1 obtenida tras la reconstrucción de uno de los locus URA3 de la cepa SFC4 $(\sin 3 \Delta)$ y ZAHR4 obtenida de la cepa ZAH4 (gcn5 $\Delta, \sin 3 \Delta$ ) para inocular por vía intraperitoneal en 10 ratones CD1 con $10^{8}$ (data no muestrado) y $5 \times 10^{8}$ células de $C$. albicans cada uno. A partir del día de infección se realizó un seguimiento diario de la supervivencia de los ratones infectados que se prolongó hasta el día 30. Se muestra que no han muerto $80 \%$ de los ratones injectado con $5 \times 10$ de las celulas de los mutante simple $\sin 3 \Delta$ y doble $g c n 5 \Delta, \sin 3 \Delta$ durante tiempo ensayadas (Fig. 3.10).

\section{Análisis del perfil transcripcional}

A la vista de los resultados obtenidos en la caracterización fenotípica de la cepa doble delecionado decidimos estudiar en detalle el patrón de transcripción de la cepa ZAH4 durante crecimiento de forma levadura y durante la transición levadura-hifa. Con el objetivo de averiguar si se detectaba una alteración en la expresión de los genes que codifican para proteínas que intervienen en la formación de hifa. Para ello, se realizaron comparaciones entre el perfil transcripcional de la transición levadura-hifa a los 15,60 y 180 minutos. De esta manera observamos que 558 genes alteraban significativamente sus expresiones durante la fase de levadura y 1271 genes cambiaban significativamente su expresión en al menos uno de los tres tiempos indicados (Fig. 3.12B).

\section{Análisis del perfil de transcripción del doble mutante $g c n 5 \Delta, \sin 3 \Delta$ durante la} fase de levadura

Dentro del grupo de genes sobreexpresados durante crecemiento de forma de levadura, las categorías y subcategorías estadísticamente significativas son respuesta a estrés oxidativo, oxidación de ácidos grasos, replicación y síntesis de ADN, transporte de azúcares y obtención de energía (principalmente a través de TCA).

Entre los genes subexpresados, las categorías más significativas son: genes relacionados con pared celular, transporte, genes que codifican para proteínas ribosomales y factores de transcripción.

Dentro de los genes sobreexpresados, se detectaron genes relacionados con alteraciones morfológicas como NUM11, NUM12, TUB1 y TUB2. Los genes NUM11 y TUB1 se han encontrado sobreexpresados en la cepa carente de SIN3 (Martin, Tesis doctoral 2006). Los productos de estos genes participan en la biogénesis y función del 
citoesqueleto afectando de este modo a la organización intracelular. Mutaciones en los genes homólogos de S. cerevisiae, ScNUM1 y ScTUB1, causan defectos en la correcta migración del núcleo (Heil-Chapdelaine et al., 2000; Schatz et al., 1988). En el caso de ScTUB1, se ha descrito que su sobreexpresión supone un bloqueo temporal del ciclo celular, durante el cual continúa el crecimiento causando un incremento del tamaño de las células (Weinstein \& Solomon, 1990). Las células mutantes doble como $\sin 3 \Delta$ son más alargadas que las silvestres, la sobreexpresión de genes del citoesqueleto que participan en organización celular y polaridad podría contribuir a este fenotipo. Se ha detectado la sobreexpresion de un número significativo de genes relacionados con el metabolismo oxidativo como GRX3, SOD2 y TRX1. Con el fin de averiguar si la expresión diferencial de estos genes tenía consecuencias biológicas se analizó la sensibilidad de la cepa doble delecionada a un agente oxidante, el peróxido de hidrógeno. A partir de células recogidas en fase exponencial de crecimiento se preparó una suspensión de $10^{7}$ células $/ \mathrm{ml}$ de cada cepa. Tras diferentes concentraciones a $\mathrm{H}_{2} \mathrm{O}_{2}$, se recogieron muestras de $5 \mu \mathrm{l}$ y se depositaron sobre placas de YEPD. En concordancia con los resultados de los microarrays, se observó que el mutante doble como mutante simple $\sin 3 \Delta$ presentaba mayor resistencia a la acción del peróxido de hidrógeno $\left(\mathrm{H}_{2} \mathrm{O}_{2}\right)$ que la cepa parental, CAI4.

\section{Análisis del transcriptoma del doble mutante $g \operatorname{cn} 5 \Delta, \sin 3 \Delta$ durante la transición} levadura-hifa

El mismo estudio de microarrays se llevó a cabo durante la transición levadura-hifas. Este análisis mostró 1271 genes alterados con 277 genes de función desconocida. 528, 891 y 146 genes expresados diferencialmente en cada uno de los tiempos analizados (Fig 3.12). El nombre y la función de cada uno de ellos se han incluido en las tablas 3.3 y 3.4 del material suplementario. En conjunto se detectó que la expresión de 1271 genes (690 sobre- y 581 sub-expresados) está afectada en el mutante doble $g c n 5 \Delta, \sin 3 \Delta .43$ genes son comunes a los tres tiempos ensayados (10 sobre- y 33 sub-expresados), 156 genes en los 15 y 60 min (102 sobre- y 54 subexpresados) y 47 genes en 60 y $180 \mathrm{~min}$. (9 sobre- y 38 sub-expresados). 324 genes (227 sobre- y 97 sub-expresados), 645 genes (315 sobre- y 330 sub-expresados) y 51 genes (24 sobre- y 27 sub-expresados) sólo varían en un tiempo de inducción (15, 60 y $180 \mathrm{~min}$ respectivamente). Considerando datos publicados por diversos autores sobre la modulación de la expresión génica durante la transición levadura-hifa, se trató de determinar que genes implicados en morfogénesis se encontraban alterados en el mutante doble gcn $5 \Delta$, $\sin 3 \Delta$. Los 43 genes comunes a los tres tiempos se muestran en la Tabla 3.4. De acuerdo a las morfología del mutante doble durante transición 
levadura-hifa, en este grupo se detecta la subexpresión de genes que codifican proteínas principalmente asociadas a la pared celular que habían sido previamente reconocidos como hifa-específicos: ECE1 (Birse et al., 1993), RBT1 (Braun et al., 2000), HWP1 (Sharkey et al., 1999), ALS1 y ALS3. Tal y como se muestra en la Tabla 3.4 de material suplementario, 3 genes que codifican las histonas $\mathrm{H} 2, \mathrm{H} 3$ y $\mathrm{H} 4$ (HHF22, HHT1 y HHT21) están subexpresados en el 60 min de la transición analizado. La subexpresión de estos genes podría estar relacionada en este mutante con el retraso de crecimiento que muestra esta cepa respecto a la cepa parental, como se ha sugerido en otros mutantes donde estos genes se encuentran subexpresados y muestran crecimiento más lento (Bensen et al., 2002). Se ha detectado la regulación de un número de genes relacionados con la pared celular incluidos: PGA1 (Castillo et al., 2006; De Groot et al., 2003), PGA49 (De Groot et al., 2003), CWH41 (Mora-Montes et al., 2007), ECM1 (Harcus et al., 2004), FGR12 (Uhl et al., 2003), FGR41 (De Groot et al., 2003), IFF5 (De Groot et al., 2003) sobreexpresados y KRE1 (Castillo et al., 2006), PGA6, PGA45, PGA53, PGA62, PGA63, ECM21, ECM33 (Martinez-Lopez et al., 2008), FGR6 y PHR1 subexpresados en 60 minuto de inducción (Fig. 3.14B). Hemos detectado la sobreexpresion de un número de los genes relacionado con proteínas ribosomales (NOC4, NOP2, NOP10, RPB8, RPC10, RPC31, RPC82, RPL25, RPL26A, RPL27A, RPL35, RRP1, RRP3, RRP9, RRS1, RSA2 y SIK1) y la subexpresion de RPL2, RPL7A, RPL13, RPL14, RPL15A, RPL18, RPL30, RPN2, $R P P 2 A, R P P 2 B, R P S 4 A, R P S 16 A, R P S 17 B$ y RPS20 genes durante 60 minutos de incubación. No hemos encontrados genes relacionados en este categoría en otros tiempos de inducción (15 y $180 \mathrm{~min}$ ). Después de 60 minutos de inducción se han observado la subexpresión de genes que codifican proteínas ribosomales en el mutante simple $\sin 3 \Delta$. Este comportamiento, subexpresión de genes implicados en procesamiento de ARN seguido de subexpresión de genes que codifican proteínas ribosomales, se corresponde con el descrito en la respuesta a diferentes tipos de estrés en S. cerevisiae (Boorsma et al., 2005; Gasch et al., 2000; Gasch et al., 2004). Parece que la subexpresión de estos genes podría estar relacionada con la sobreexpresión de genes de respuesta a estrés oxidativo y sobre todo de la activación de la respuesta a carencia de aminoácidos. Se ha demostrado que la inducción de ScGCN4 conlleva la subexpresión de genes que codifican proteínas ribosomales (Natarajan et al., 2001). En el capitulo III, se ha explicado con más detalles algunos grupos de genes cuya expresión ha sido modificada por la deleción de GCN5 y SIN3. 


\section{Conclusiones}

1. Se ha llevado a cabo la deleción del gen CaHAT1 en la cepa RDH4 (hat2A). La ausencia de ambas proteínas tiene un efecto en morfogénesis, ya que el doble mutante hat $1 \Delta$, hat $2 \Delta$ presenta defectos similares a los del mutante sencillo hat $1 \Delta$ : morfología de pseudohifa cuando crecen a $28^{\circ} \mathrm{C}$, defectos de filamentación y formación de pseudohifas en lugar de hifas en medios líquidos en condiciones de inducción.

2. Se ha observado que los mutantes hat $1 \Delta$, hat $2 \Delta$ y hat $1 \Delta$ muestran sensibilidad a $\mathrm{NaCl}$, Rojo congo, Calcoflour white y Cafeína. Tanto el mutante doble como el mutante simple hat1 $\Delta$, también mostraron sensibilidad moderada frente a compuestos como Fluconazol, Itraconazol, Higromicina y Anfotericina B.

3. Se observó que las proteínas Hat1p y Hat2p se localizan en el núcleo en $C$. albicans. Además, se observó que en el mutante hat1 $\Delta$, la proteína Hat2p, no se localiza en el núcleo. La localización de la proteína Hat2p es dependiente de la proteína Hat1p. Estos resultados son contrarios a los observados en $S$. cerevisiae.

4. Se ha comparado el transcriptoma del mutante simple hat $2 \Delta$ con el de la cepa parental CAI4, observándose que: en fase exponencial de crecimiento a $28^{\circ} \mathrm{C}$, en la cepa delecionada se encuentra alterada la expresión de 109 genes, considerando un factor de variación $\geq 1.5$ veces. Entre estos genes, aparecen subexpresados genes implicados en respiración, ensamblaje de histonas y subexpresados genes implicados en la ruta de glicólisis, TCA, biosíntesis de ribosomas, pared celular y respuesta a estrés. Durante la transición dimórfica, aparece alterada la expresión de 429 genes. Las principales categorías asociadas a sobreexpresión son: metabolismo de carbohidratos (glicólisis), pared celular, transporte de glucosa, genes relacionados con la familia de la telomerasa y proteínas ribosomales. Entre los genes subexpresados se encuentran principalmente genes que codifican proteínas relacionadas con la maquinaria de transcripción (factores de trascripción), el procesamiento de rARN, respiración, pared celular y biosíntesis de ergosterol.

5. Se ha comparado el transcriptoma del mutante doble hat $1 \Delta$, hat $2 \Delta$ con el de la cepa parental CAI4, observándose que: en fase exponencial de crecimiento a $28^{\circ} \mathrm{C}$, en la cepa delecionada se encuentra alterada la expresión de 219 genes, considerando un factor de variación $\geq 1.5$ veces. Entre estos genes, aparecen sobrexpresados genes implicados en pared celular, respuesta a estrés, genes relacionados con proteosoma, 5 genes relacionados con la familia de la telomerasa. Tres genes de esta familia son comunes entre el mutante doble y hat1 $\Delta$ lo que sugería que Hat1p interviene en el silenciamiento telomérico. Entre estos genes, aparecen subexpresados genes implicados en ruta de glicólisis, TCA y transportadores. 
Durante la transición dimórfica, aparece alterada la expresión de 794 genes. Las principales categorías asociadas a sobreexpresión son respuesta a estrés, procesamiento de rARN y síntesis de proteínas, pared celular y 6 genes relacionados con el complejo del proteosoma (NOP1, NOP2, NOP4, NOP5, NOP14 y NOP15). Se ha detectado la subexpresión secuencial de genes relacionados con pared celular, glicólisis, ruta de secreción (SEC9, SEC11, SEC16, SEC23, SEC24, SEC26 y SEC61), resistencia a drogas (CDR1, CDR2, CDR4 y QDR1) y 14 genes relacionados con factores de transcripción.

6. Se ha llevado a cabo la deleción del gen CaSIN3 en la cepa RDG4 (gcn54). La ausencia de ambas proteínas tiene un efecto intermedio en morfogénesis: pseudohifa y morfología alargada de las levaduras cuando crecen a $28^{\circ} \mathrm{C}$, defectos de filamentación y formación de pseudohifas en lugar de hifas en medios líquidos de inducción e incapacidad para formar clamidosporas.

7. Se comprobó que el mutante simple $\sin 3 \Delta$ y doble $g \operatorname{cn} 5 \Delta, \sin 3 \Delta$ afectan a la virulencia de $C$. albicans en un modelo de infección sistémica de ratón.

8. Se ha observado que tanto el mutante $g c n 5 \Delta, \sin 3 \Delta$ como el mutante simple gcn5 $\Delta$ muestran sensibilidad a los compuestos como $\mathrm{NaCl}$, Rojo congo, $\mathrm{KCl}, \mathrm{CaCl}_{2}$, Higromicina y Cafeína.

9. La comparación del perfil transcripcional completo del mutante doble gcn5 $5, \sin 3 \Delta$ con el de la cepa parental CAI4 indica que:

- En fase exponencial de crecimiento a $28^{\circ} \mathrm{C}$ la deleción de GCN5 y SIN3 altera la expresión 558 genes que entre ellos 294 genes sobre- y 264 genes subexpresados. Entre ellos se identificaron sobreexpresados varios genes relacionados con síntesis y replicación de ADN, genes que codifican para factores transcripcionales, genes relacionados con estructura celular como TUP1, NUM11, pared celular y ciclo de TCA. Las principales categorías asociadas a sobreexpresión son pared celular (CWH41, ECM1, FGR12, FGR41, IFF5, PGA1 y PGA49) (60 min), 17 genes que codifican proteínas ribosomales y genes relacionados con factores de transcripción, incluyendo el gen que codifica el activador transcripcional Gcn4p. Se ha detectado la subexpresión secuencial de genes relacionados con filamentación, genes que codifican factores transcripción y proteínas relacionadas con pared celular, genes que codifican proteínas ribosomales, genes relacionados con biosíntesis de vitaminas, ruta de glicólisis y además genes que codifican para proteínas estructurales de las hifas. La subexpresión de genes que codifican para proteínas ribosomales y otros factores relacionados con funciones generales de crecimiento, reflejan una respuesta en el mutante $g c n 5 \Delta, \sin 3 \Delta$ al retraso en el crecimiento.

10. Durante la transición dimórfica, aparece alterada la expresión de 1271 genes.Las principales categorías asociadas a sobreexpresión son metabolismo de aminoácidos, 
pared celular, factores de transcripción incluyendo el gen que pared celular, factores de transcripción incluyendo el gen que codifica el activador transcripcional Gcn4p.

11. Se ha observado que el nivel de expresión de los genes relacionados con pared celular como ALS1, ALS3, MNT1, PGA53, PGA54, PGA45, HYR1, ECM33, TOS1, PMT1 y SUN41, fue disminuyendo a la largo de transición levadura-hifa en los mutantes simples $g c n 5 \Delta, \sin 3 \Delta$ y en el mutante doble $g c n 5 \Delta, \sin 3 \Delta$, lo que sugiere que estos genes están regulados por separado por ambos genes.

12. Se ha observado que el nivel de expresión de los genes relacionados con pared celular como PHR1, YWP1, PGA62 y KRE1 aumentan en el mutante simple gcn5D y disminuyendo a la largo de transición levadura-hifa en los mutantes $\sin 3 \Delta$ y doble indicando que estos genes se regulan independientemente por ambos genes.

13. Se ha observado que el nivel de expresión de los genes relacionados con metabolismo de carbohidrate como TPI1, TPS2, PGK1, TDH3, OSM1, GSY1, FBA1, $A D H 5, P G / 1$ y $P D C 11$ fue disminuyendo durante 60 y 180 de de transición levadurahifa en el mutante simple $g c n 5 \Delta$ y doble $g c n 5 \Delta, \sin 3 \Delta$. 
Acikgoz, Z. C., Sancak, B., Gamberzade, S. \& Misirlioglu, M. (2004). Prevalence of Candida dubliniensis among the stored vaginal Candida isolates in a Turkish hospital. Mycoses 47, 393396.

Adam, A. L., Kohut, G. \& Hornok, L. (2008). Fphog1, a HOG-type MAP kinase gene, is involved in multistress response in Fusarium proliferatum. J Basic Microbiol 48, 151-159.

Ai, X. \& Parthun, M. R. (2004). The nuclear Hat1p/Hat2p complex: a molecular link between type B histone acetyltransferases and chromatin assembly. Mol Cell 14, 195-205.

Al-Hedaithy, S. S. \& Fotedar, R. (2002). Recovery and studies on chlamydospore-negative Candida albicans isolated from clinical specimens. Med Mycol 40, 301-306.

Al Mosaid, A., Sullivan, D. J. \& Coleman, D. C. (2003). Differentiation of Candida dubliniensis from Candida albicans on Pal's agar. J Clin Microbiol 41, 4787-4789.

Alarco, A. M., Balan, I., Talibi, D., Mainville, N. \& Raymond, M. (1997). AP1-mediated multidrug resistance in Saccharomyces cerevisiae requires FLR1 encoding a transporter of the major facilitator superfamily. J Biol Chem 272, 19304-19313.

Alarco, A. M. \& Raymond, M. (1999). The bZip transcription factor Cap1p is involved in multidrug resistance and oxidative stress response in Candida albicans. $J$ Bacteriol 181, 700708.

Alex, L. A., Korch, C., Selitrennikoff, C. P. \& Simon, M. I. (1998). COS1, a two-component histidine kinase that is involved in hyphal development in the opportunistic pathogen Candida albicans. Proc Natl Acad Sci U S A 95, 7069-7073.

Alonso-Monge, R., Navarro-Garcia, F., Roman, E., Negredo, A. I., Eisman, B., Nombela, C. \& Pla, J. (2003). The Hog1 mitogen-activated protein kinase is essential in the oxidative stress response and chlamydospore formation in Candida albicans. Eukaryot Cell 2, 351-361.

Allfrey, V. G., Faulkner, R. \& Mirsky, A. E. (1964). Acetylation and Methylation of Histones and Their Possible Role in the Regulation of Rna Synthesis. Proc Natl Acad Sci U S A 51, 786794.

Anderson, J. M. \& Soll, D. R. (1986). Differences in actin localization during bud and hypha formation in the yeast Candida albicans. J Gen Microbiol 132, 2035-2047.

Arga, K. Y., Onsan, Z. I., Kirdar, B., Ulgen, K. O. \& Nielsen, J. (2007). Understanding signaling in yeast: Insights from network analysis. Biotechnol Bioeng 97, 1246-1258.

Aridogan, I. A., Ilkit, M., Izol, V. \& Ates, A. (2005). Malassezia and Candida colonisation on glans penis of circumcised men. Mycoses 48, 352-356.

Backen, A. C., Broadbent, I. D., Fetherston, R. W., Rosamond, J. D., Schnell, N. F. \& Stark, M. J. (2000). Evaluation of the CaMAL2 promoter for regulated expression of genes in Candida albicans. Yeast 16, 1121-1129.

Bachewich, C., Thomas, D. Y. \& Whiteway, M. (2003). Depletion of a polo-like kinase in Candida albicans activates cyclase-dependent hyphal-like growth. Mol Biol Cell 14, 2163-2180.

Bachewich, C. \& Whiteway, M. (2005). Cyclin CIn3p links G1 progression to hyphal and pseudohyphal development in Candida albicans. Eukaryot Cell 4, 95-102.

Bader, O., Krauke, Y. \& Hube, B. (2008). Processing of predicted substrates of fungal Kex2 proteinases from Candida albicans, C. glabrata, Saccharomyces cerevisiae and Pichia pastoris. BMC microbiology 8, 116. 
Bailey, D. A., Feldmann, P. J., Bovey, M., Gow, N. A. \& Brown, A. J. (1996). The Candida albicans HYR1 gene, which is activated in response to hyphal development, belongs to a gene family encoding yeast cell wall proteins. J Bacteriol 178, 5353-5360.

Baker, S. P. \& Grant, P. A. (2007). The SAGA continues: expanding the cellular role of a transcriptional co-activator complex. Oncogene 26, 5329-5340.

Bakkenist, C. J. \& Kastan, M. B. (2004). Initiating cellular stress responses. Cell 118, 9-17.

Banerjee, M., Thompson, D. S., Lazzell, A., Carlisle, P. L., Pierce, C., Monteagudo, C., Lopez-Ribot, J. L. \& Kadosh, D. (2008). UME6, a novel filament-specific regulator of Candida albicans hyphal extension and virulence. Mol Biol Cell 19, 1354-1365.

Barabino, S. M., Ohnacker, M. \& Keller, W. (2000). Distinct roles of two Yth1p domains in 3'end cleavage and polyadenylation of yeast pre-mRNAs. Embo J 19, 3778-3787.

Barelle, C. J., Bohula, E. A., Kron, S. J., Wessels, D., Soll, D. R., Schafer, A., Brown, A. J. \& Gow, N. A. (2003). Asynchronous cell cycle and asymmetric vacuolar inheritance in true hyphae of Candida albicans. Eukaryot Cell 2, 398-410.

Barelle, C. J., Manson, C. L., MacCallum, D. M., Odds, F. C., Gow, N. A. \& Brown, A. J. (2004). GFP as a quantitative reporter of gene regulation in Candida albicans. Yeast (Chichester, England) 21, 333-340.

Barros, L. M., Boriollo, M. F., Alves, A. C., Klein, M. I., Goncalves, R. B. \& Hofling, J. F. (2008). Genetic diversity and exoenzyme activities of Candida albicans and Candida dubliniensis isolated from the oral cavity of Brazilian periodontal patients. Arch Oral Biol 53, 1172-1178.

Bassilana, M., Blyth, J. \& Arkowitz, R. A. (2003). Cdc24, the GDP-GTP exchange factor for Cdc42, is required for invasive hyphal growth of Candida albicans. Eukaryot Cell 2, 9-18.

Bassilana, M., Hopkins, J. \& Arkowitz, R. A. (2005). Regulation of the Cdc42/Cdc24 GTPase module during Candida albicans hyphal growth. Eukaryot Cell 4, 588-603.

Bassilana, M. \& Arkowitz, R. A. (2006). Rac1 and Cdc42 have different roles in Candida albicans development. Eukaryot Cell 5, 321-329.

Bates, S., de la Rosa, J. M., MacCallum, D. M., Brown, A. J., Gow, N. A. \& Odds, F. C. (2007). Candida albicans Iff11, a secreted protein required for cell wall structure and virulence. Infect Immun 75, 2922-2928.

Bennett, R. J., UhI, M. A., Miller, M. G. \& Johnson, A. D. (2003). Identification and characterization of a Candida albicans mating pheromone. Mol Cell Biol 23, 8189-8201.

Bensen, E. S., Filler, S. G. \& Berman, J. (2002). A forkhead transcription factor is important for true hyphal as well as yeast morphogenesis in Candida albicans. Eukaryot Cell 1, 787-798.

Bensen, E. S., Martin, S. J., Li, M., Berman, J. \& Davis, D. A. (2004). Transcriptional profiling in Candida albicans reveals new adaptive responses to extracellular $\mathrm{pH}$ and functions for Rim101p. Mol Microbiol 54, 1335-1351.

Bensen, E. S., Clemente-Blanco, A., Finley, K. R., Correa-Bordes, J. \& Berman, J. (2005). The mitotic cyclins Clb2p and Clb4p affect morphogenesis in Candida albicans. Mol Biol Cell 16, 3387-3400.

Benson, L. J., Gu, Y., Yakovleva, T., Tong, K., Barrows, C., Strack, C. L., Cook, R. G., Mizzen, C. A. \& Annunziato, A. T. (2006). Modifications of $\mathrm{H} 3$ and $\mathrm{H} 4$ during chromatin replication, nucleosome assembly, and histone exchange. J Biol Chem 281, 9287-9296. 
Berger, S. L. (2007). The complex language of chromatin regulation during transcription. Nature 447, 407-412.

Berman, J. \& Sudbery, P. E. (2002). Candida Albicans: a molecular revolution built on lessons from budding yeast. Nat Rev Genet 3, 918-930.

Berman, J. G., N. (2004). Cell cycle. In Pathogenic fungi Structural Biology and taxonomy. . England: Caister Academic Press.

: G. San-Blas \& R. Calderone.

Birse, C. E., Irwin, M. Y., Fonzi, W. A. \& Sypherd, P. S. (1993). Cloning and characterization of ECE1, a gene expressed in association with cell elongation of the dimorphic pathogen Candida albicans. Infect Immun 61, 3648-3655.

Biswas, S., Van Dijck, P. \& Datta, A. (2007). Environmental sensing and signal transduction pathways regulating morphopathogenic determinants of Candida albicans. Microbiol Mol Biol Rev 71, 348-376.

Boorsma, A., Foat, B. C., Vis, D., Klis, F. \& Bussemaker, H. J. (2005). T-profiler: scoring the activity of predefined groups of genes using gene expression data. Nucleic Acids Res 33, W592-595.

Brand, A., MacCallum, D. M., Brown, A. J., Gow, N. A. \& Odds, F. C. (2004). Ectopic expression of URA3 can influence the virulence phenotypes and proteome of Candida albicans but can be overcome by targeted reintegration of URA3 at the RPS10 locus. Eukaryot Cell 3, 900-909.

Braun, B. R. \& Johnson, A. D. (1997). Control of filament formation in Candida albicans by the transcriptional repressor TUP1. Science 277, 105-109.

Braun, B. R., Head, W. S., Wang, M. X. \& Johnson, A. D. (2000). Identification and characterization of TUP1-regulated genes in Candida albicans. Genetics 156, 31-44.

Braun, B. R., Kadosh, D. \& Johnson, A. D. (2001). NRG1, a repressor of filamentous growth in C.albicans, is down-regulated during filament induction. Embo $\mathrm{J}$ 20, 4753-4761.

Braun, B. R., van Het Hoog, M., d'Enfert, C. \& other authors (2005). A human-curated annotation of the Candida albicans genome. PLoS Genet 1, 36-57.

Brown, D. H., Jr., Giusani, A. D., Chen, X. \& Kumamoto, C. A. (1999). Filamentous growth of Candida albicans in response to physical environmental cues and its regulation by the unique CZF1 gene. Mol Microbiol 34, 651-662.

Brown, V., Sexton, J. A. \& Johnston, M. (2006). A glucose sensor in Candida albicans. Eukaryot Cell 5, 1726-1737.

Brownell, J. E. \& Allis, C. D. (1996). Special HATs for special occasions: linking histone acetylation to chromatin assembly and gene activation. Curr Opin Genet Dev 6, 176-184.

Bruno, V. M. \& Mitchell, A. P. (2005). Regulation of azole drug susceptibility by Candida albicans protein kinase CK2. Mol Microbiol 56, 559-573.

Buffo, J., Herman, M. A. \& Soll, D. R. (1984). A characterization of pH-regulated dimorphism in Candida albicans. Mycopathologia 85, 21-30.

Buschelman, B., Jones, R. N., Pfaller, M. A., Koontz, F. P. \& Doern, G. V. (1999). Colony morphology of Candida spp. as a guide to species identification. Diagn Microbiol Infect Dis $\mathbf{3 5}$, 89-91. 
Buurman, E. T., Westwater, C., Hube, B., Brown, A. J., Odds, F. C. \& Gow, N. A. (1998). Molecular analysis of CaMnt1p, a mannosyl transferase important for adhesion and virulence of Candida albicans. Proc Natl Acad Sci U S A 95, 7670-7675.

Calderone, R., Suzuki, S., Cannon, R. \& other authors (2000). Candida albicans: adherence, signaling and virulence. Med Mycol 38 Suppl 1, 125-137.

Calderone, R. (2002). Candida and Candidiasis. Washington: ASM press.

Calderone, R. A. \& Fonzi, W. A. (2001). Virulence factors of Candida albicans. Trends Microbiol 9, 327-335.

Campa, D., Tavanti, A., Gemignani, F., Mogavero, C. S., Bellini, I., Bottari, F., Barale, R., Landi, S. \& Senesi, S. (2008). DNA microarray based on arrayed-primer extension technique for identification of pathogenic fungi responsible for invasive and superficial mycoses. $J$ Clin Microbiol 46, 909-915.

Cano, A. \& Pestana, A. (1979). Purification and properties of a histone acetyltransferase from Artemia salina, highly efficient with $\mathrm{H} 1$ histone. Eur J Biochem 97, 65-72.

Cantero, P. D., Lengsfeld, C., Prill, S. K., Subanovic, M., Roman, E., Pla, J. \& Ernst, J. F. (2007). Transcriptional and physiological adaptation to defective protein-O-mannosylation in Candida albicans. Mol Microbiol 64, 1115-1128.

Cao, Y. Y., Cao, Y. B., Xu, Z., Ying, K., Li, Y., Xie, Y., Zhu, Z. Y., Chen, W. S. \& Jiang, Y. Y. (2005). cDNA microarray analysis of differential gene expression in Candida albicans biofilm exposed to farnesol. Antimicrob Agents Chemother 49, 584-589.

Care, R. S., Trevethick, J., Binley, K. M. \& Sudbery, P. E. (1999). The MET3 promoter: a new tool for Candida albicans molecular genetics. Mol Microbiol 34, 792-798.

Carlisle, P. L., Banerjee, M., Lazzell, A., Monteagudo, C., Lopez-Ribot, J. L. \& Kadosh, D. (2009). Expression levels of a filament-specific transcriptional regulator are sufficient to determine Candida albicans morphology and virulence. Proceedings of the National Academy of Sciences of the United States of America 106, 599-604.

Carroll, S. B. (2000). Endless forms: the evolution of gene regulation and morphological diversity. Cell 101, 577-580.

Carrozza, M. J., Utley, R. T., Workman, J. L. \& Cote, J. (2003). The diverse functions of histone acetyltransferase complexes. Trends Genet 19, 321-329.

Carrozza, M. J., Utley, R. T., Workman, J. L. y Côté, J. (2003). The diverse functions of histone acetyltransferase complexes. Trends Genet 19, 321-329.

Castilla, R., Passeron, S. \& Cantore, M. L. (1998). N-acetyl-D-glucosamine induces germination in Candida albicans through a mechanism sensitive to inhibitors of cAMPdependent protein kinase. Cell Signal 10, 713-719.

Castillo, L., Martinez, A. I., Garcera, A., Garcia-Martinez, J., Ruiz-Herrera, J., Valentin, E. \& Sentandreu, R. (2006). Genomic response programs of Candida albicans following protoplasting and regeneration. Fungal Genet Biol 43, 124-134.

Castillo, L., Calvo, E., Martinez, A. I., Ruiz-Herrera, J., Valentin, E., Lopez, J. A. \& Sentandreu, R. (2008). A study of the Candida albicans cell wall proteome. Proteomics 8 , 3871-3881. 
Cole, G. T., Seshan, K. R., Phaneuf, M. \& Lynn, K. T. (1991). Chlamydospore-like cells of Candida albicans in the gastrointestinal tract of infected, immunocompromised mice. Can $J$ Microbiol 37, 637-646.

Cole, M. F., Bowen, W. H., Zhao, X. J. \& Cihlar, R. L. (1995). Avirulence of Candida albicans auxotrophic mutants in a rat model of oropharyngeal candidiasis. FEMS Microbiol Lett 126, 177180.

Copping, V. M., Barelle, C. J., Hube, B., Gow, N. A., Brown, A. J. \& Odds, F. C. (2005). Exposure of Candida albicans to antifungal agents affects expression of SAP2 and SAP9 secreted proteinase genes. J Antimicrob Chemother 55, 645-654.

Cormack, B. P., Bertram, G., Egerton, M., Gow, N. A., Falkow, S. \& Brown, A. J. (1997). Yeast-enhanced green fluorescent protein (yEGFP)a reporter of gene expression in Candida albicans. Microbiology (Reading, England) 143 ( Pt 2), 303-311.

Cress, W. D. \& Seto, E. (2000). Histone deacetylases, transcriptional control, and cancer. J Cell Physiol 184, 1-16.

Csank, C., Schroppel, K., Leberer, E., Harcus, D., Mohamed, O., Meloche, S., Thomas, D. Y. \& Whiteway, M. (1998). Roles of the Candida albicans mitogen-activated protein kinase homolog, Cek1p, in hyphal development and systemic candidiasis. Infect Immun 66, 27132721.

Cutler, J. E. (1991). Putative virulence factors of Candida albicans. Annu Rev Microbiol 45, 187-218.

Chabrier-Rosello, Y., Foster, T. H., Mitra, S. \& Haidaris, C. G. (2008). Respiratory deficiency enhances the sensitivity of the pathogenic fungus Candida to photodynamic treatment. Photochem Photobiol 84, 1141-1148.

Chaffin, W. L. (1984). The relationship between yeast cell size and cell division in Candida albicans. Can J Microbiol 30, 192-203.

Chen, J., Chen, J., Lane, S. \& Liu, H. (2002). A conserved mitogen-activated protein kinase pathway is required for mating in Candida albicans. Mol Microbiol 46, 1335-1344.

Chen, R. E. \& Thorner, J. (2007). Function and regulation in MAPK signaling pathways: lessons learned from the yeast Saccharomyces cerevisiae. Biochim Biophys Acta 1773, 13111340.

Cheng, S., Clancy, C. J., Checkley, M. A., Handfield, M., Hillman, J. D., Progulske-Fox, A., Lewin, A. S., Fidel, P. L. \& Nguyen, M. H. (2003). Identification of Candida albicans genes induced during thrush offers insight into pathogenesis. Mol Microbiol 48, 1275-1288.

Chibana, H., Iwaguchi, S., Homma, M., Chindamporn, A., Nakagawa, Y. \& Tanaka, K. (1994). Diversity of tandemly repetitive sequences due to short periodic repetitions in the chromosomes of Candida albicans. J Bacteriol 176, 3851-3858.

Chibana, H., Beckerman, J. L. \& Magee, P. T. (2000). Fine-resolution physical mapping of genomic diversity in Candida albicans. Genome Res 10, 1865-1877.

Chibana, H., Oka, N., Nakayama, H., Aoyama, T., Magee, B. B., Magee, P. T. \& Mikami, Y. (2005). Sequence finishing and gene mapping for Candida albicans chromosome 7 and syntenic analysis against the Saccharomyces cerevisiae genome. Genetics 170, 1525-1537.

Chio, T. (2004 ). Morphogenesis in C. albicans. In Pathogenic fungi Structural Biology and taxonomy. England: Caister Academic Press.: Edited by G. San-Blas \& R. Calderone. 
d'Enfert, C., Goyard, S., Rodriguez-Arnaveilhe, S. \& other authors (2005). CandidaDB: a genome database for Candida albicans pathogenomics. Nucleic Acids Res 33, D353-357.

Darwazeh, A. M., Lamey, P. J., Samaranayake, L. P., MacFarlane, T. W., Fisher, B. M., Macrury, S. M. \& MacCuish, A. C. (1990). The relationship between colonisation, secretor status and in-vitro adhesion of Candida albicans to buccal epithelial cells from diabetics. $J$ Med Microbiol 33, 43-49.

David, G., Grandinetti, K. B., Finnerty, P. M., Simpson, N., Chu, G. C. \& Depinho, R. A. (2008). Specific requirement of the chromatin modifier $\mathrm{mSin} 3 \mathrm{~B}$ in cell cycle exit and cellular differentiation. Proc Natl Acad Sci U S A 105, 4168-4172.

Davis-Hanna, A., Piispanen, A. E., Stateva, L. I. \& Hogan, D. A. (2008). Farnesol and dodecanol effects on the Candida albicans Ras1-cAMP signalling pathway and the regulation of morphogenesis. Mol Microbiol 67, 47-62.

Davis, D. (2003). Adaptation to environmental $\mathrm{pH}$ in Candida albicans and its relation to pathogenesis. Curr Genet 44, 1-7.

Davis, D. A., Bruno, V. M., Loza, L., Filler, S. G. \& Mitchell, A. P. (2002). Candida albicans Mds3p, a conserved regulator of $\mathrm{pH}$ responses and virulence identified through insertional mutagenesis. Genetics 162, 1573-1581.

De Groot, P. W., Hellingwerf, K. J. \& Klis, F. M. (2003). Genome-wide identification of fungal GPI proteins. Yeast 20, 781-796.

de Groot, P. W., de Boer, A. D., Cunningham, J., Dekker, H. L., de Jong, L., Hellingwerf, K. J., de Koster, C. \& Klis, F. M. (2004). Proteomic analysis of Candida albicans cell walls reveals covalently bound carbohydrate-active enzymes and adhesins. Eukaryot Cell 3, 955-965.

de la Rosa, J. M., Ruiz, T., Fonzi, W. A. \& Rodriguez, L. (2004). Analysis of heterologous expression of Candida albicans SEC61 gene reveals differences in Sec61p homologues related to species-specific functionality. Fungal Genet Biol 41, 941-953.

Decker, R. S., Yamaguchi, M., Possenti, R., Bradley, M. K. \& DePamphilis, M. L. (1987). In vitro initiation of DNA replication in simian virus 40 chromosomes. $J$ Biol Chem 262, 1086310872.

Delbruck, S. \& Ernst, J. F. (1993). Morphogenesis-independent regulation of actin transcript levels in the pathogenic yeast Candida albicans. Mol Microbiol 10, 859-866.

DeSilva, H., Lee, K. \& Osley, M. A. (1998). Functional dissection of yeast Hir1p, a WD repeatcontaining transcriptional corepressor. Genetics 148, 657-667.

Dhillon, N. K., Sharma, S. \& Khuller, G. K. (2003). Signaling through protein kinases and transcriptional regulators in Candida albicans. Crit Rev Microbiol 29, 259-275.

Doedt, T., Krishnamurthy, S., Bockmuhl, D. P., Tebarth, B., Stempel, C., Russell, C. L., Brown, A. J. \& Ernst, J. F. (2004). APSES proteins regulate morphogenesis and metabolism in Candida albicans. Mol Biol Cell 15, 3167-3180.

DohIman, H. G. \& Slessareva, J. E. (2006). Pheromone signaling pathways in yeast. Sci STKE 2006, cm6.

Dominic, R. M., Shenoy, S. \& Baliga, S. (2007). Candida biofilms in medical devices: evolving trends. Kathmandu Univ Med J (KUMJ) 5, 431-436. 
Dunkler, A., Walther, A., Specht, C. A. \& Wendland, J. (2005). Candida albicans CHT3 encodes the functional homolog of the Cts1 chitinase of Saccharomyces cerevisiae. Fungal Genet Biol 42, 935-947.

Eisenhaber, B., Schneider, G., Wildpaner, M. \& Eisenhaber, F. (2004). A sensitive predictor for potential GPI lipid modification sites in fungal protein sequences and its application to genome-wide studies for Aspergillus nidulans, Candida albicans, Neurospora crassa, Saccharomyces cerevisiae and Schizosaccharomyces pombe. J Mol Biol 337, 243-253.

Ekwall, K. (2005). Genome-wide analysis of HDAC function. Trends Genet 21, 608-615.

Engebrecht, J. (2003). Cell signaling in yeast sporulation. Biochem Biophys Res Commun 306 , 325-328.

Enjalbert, B. \& Whiteway, M. (2005). Release from quorum-sensing molecules triggers hyphal formation during Candida albicans resumption of growth. Eukaryot Cell 4, 1203-1210.

Enjalbert, B., Smith, D. A., Cornell, M. J., Alam, I., Nicholls, S., Brown, A. J. \& Quinn, J. (2006). Role of the Hog1 stress-activated protein kinase in the global transcriptional response to stress in the fungal pathogen Candida albicans. Mol Biol Cell 17, 1018-1032.

Ernst, J. F. (2000a). Regulation of dimorphism in Candida albicans. Contrib Microbio/ 5, 98111.

Ernst, J. F. (2000b). Transcription factors in Candida albicans - environmental control of morphogenesis. Microbiology 146 ( Pt 8), 1763-1774.

Escote, X., Zapater, M., Clotet, J. \& Posas, F. (2004). Hog1 mediates cell-cycle arrest in G1 phase by the dual targeting of Sic1. Nat Cell Biol 6, 997-1002.

Fan, J., Chaturvedi, V. \& Shen, S. H. (2002). Identification and phylogenetic analysis of a glucose transporter gene family from the human pathogenic yeast Candida albicans. $J$ Mol Evol 55, 336-346.

Fazzio, T. G., Kooperberg, C., Goldmark, J. P., Neal, C., Basom, R., Delrow, J. \& Tsukiyama, T. (2001). Widespread collaboration of Isw2 and Sin3-Rpd3 chromatin remodeling complexes in transcriptional repression. Mol Cell Biol 21, 6450-6460.

Feng, Q., Summers, E., Guo, B. \& Fink, G. (1999). Ras signaling is required for seruminduced hyphal differentiation in Candida albicans. J Bacteriol 181, 6339-6346.

Fernandez-Arenas, E., Cabezon, V., Bermejo, C., Arroyo, J., Nombela, C., Diez-Orejas, R. \& Gil, C. (2007). Integrated proteomics and genomics strategies bring new insight into Candida albicans response upon macrophage interaction. Mol Cell Proteomics 6, 460-478.

Firon, A., Aubert, S., Iraqui, I., Guadagnini, S., Goyard, S., Prevost, M. C., Janbon, G. \& d'Enfert, C. (2007). The SUN41 and SUN42 genes are essential for cell separation in Candida albicans. Mol Microbiol 66, 1256-1275.

Fonzi, W. A. \& Irwin, M. Y. (1993). Isogenic strain construction and gene mapping in Candida albicans. Genetics 134, 717-728.

Fonzi, W. A. (2002). Role of pH response in Candida albicans virulence. Mycoses 45 Suppl 1, 16-21.

Fotedar, R. \& Al Hedaithy, S. S. (2004). Prevalence of Candida dubliniensis among germ tube-positive yeasts recovered from the respiratory specimens in HIV-negative patients. Mycoses 47, 150-155. 
Fradin, C., De Groot, P., MacCallum, D., Schaller, M., Klis, F., Odds, F. C. \& Hube, B. (2005). Granulocytes govern the transcriptional response, morphology and proliferation of Candida albicans in human blood. Mol Microbiol 56, 397-415.

Gale, C., Gerami-Nejad, M., McClellan, M., Vandoninck, S., Longtine, M. S. \& Berman, J. (2001). Candida albicans Int1p interacts with the septin ring in yeast and hyphal cells. Mol Biol Cell 12, 3538-3549.

Garcia-Sanchez, S., Aubert, S., Iraqui, I., Janbon, G., Ghigo, J. M. \& d'Enfert, C. (2004). Candida albicans biofilms: a developmental state associated with specific and stable gene expression patterns. Eukaryot Cell 3, 536-545.

Garcia-Sanchez, S., Mavor, A. L., Russell, C. L., Argimon, S., Dennison, P., Enjalbert, B. \& Brown, A. J. (2005). Global roles of Ssn6 in Tup1- and Nrg1-dependent gene regulation in the fungal pathogen, Candida albicans. Mol Biol Cell 16, 2913-2925.

Gasch, A. P., Spellman, P. T., Kao, C. M., Carmel-Harel, O., Eisen, M. B., Storz, G., Botstein, D. \& Brown, P. O. (2000). Genomic expression programs in the response of yeast cells to environmental changes. Mol Biol Cell 11, 4241-4257.

Gasch, A. P., Moses, A. M., Chiang, D. Y., Fraser, H. B., Berardini, M. \& Eisen, M. B. (2004). Conservation and evolution of cis-regulatory systems in ascomycete fungi. PLoS Biol 2 , e398.

Georgakopoulos, T. y. T., G. (1992). Two distinct yeast transcriptional activators require the function of the GCN5 protein to promote normal levels of transcription. EMBO J 11, 4145-4152.

Gerami-Nejad, M., Berman, J. \& Gale, C. A. (2001). Cassettes for PCR-mediated construction of green, yellow, and cyan fluorescent protein fusions in Candida albicans. Yeast (Chichester, England) 18, 859-864.

Gimeno, C. J., Ljungdahl, P. O., Styles, C. A. \& Fink, G. R. (1992). Unipolar cell divisions in the yeast $\mathrm{S}$. cerevisiae lead to filamentous growth: regulation by starvation and RAS. Cell $\mathbf{6 8}$, 1077-1090.

Gompel, N., Prud'homme, B., Wittkopp, P. J., Kassner, V. A. \& Carroll, S. B. (2005). Chance caught on the wing: cis-regulatory evolution and the origin of pigment patterns in Drosophila. Nature 433, 481-487.

Gonzalez-Novo, A., Jimenez, J., Garcia, M. J., Rios-Serrano, I., Pla, J., Jimenez, A. \& Sanchez-Perez, M. (2004). Dynamics of $\mathrm{CaCdc} 10$, a septin of Candida albicans, in living cells and during infection. Int Microbiol 7, 105-112.

Gow, N. A., Henderson, G. \& Gooday, G. W. (1986). Cytological interrelationships between the cell cycle and duplication cycle of Candida albicans. Microbios 47, 97-105.

Gow, N. A. (1997). Germ tube growth of Candida albicans. Curr Top Med Mycol 8, 43-55.

Gow, N. A., Brown, A. J. \& Odds, F. C. (2002). Fungal morphogenesis and host invasion. Curr Opin Microbiol 5, 366-371.

Grant, P. A., Duggan, L., Cote, J. \& other authors (1997). Yeast Gcn5 functions in two multisubunit complexes to acetylate nucleosomal histones: characterization of an Ada complex and the SAGA (Spt/Ada) complex. Genes Dev 11, 1640-1650.

Grozinger, C. M. \& Schreiber, S. L. (2002). Deacetylase enzymes: biological functions and the use of small-molecule inhibitors. Chem Biol 9, 3-16. 
Gustafsson, C. M., Myers, L. C., Beve, J., Spahr, H., Lui, M., Erdjument-Bromage, H., Tempst, P. \& Kornberg, R. D. (1998). Identification of new mediator subunits in the RNA polymerase II holoenzyme from Saccharomyces cerevisiae. J Biol Chem 273, 30851-30854.

Harcus, D., Nantel, A., Marcil, A., Rigby, T. \& Whiteway, M. (2004). Transcription profiling of cyclic AMP signaling in Candida albicans. Mol Biol Cell 15, 4490-4499.

Hawser, S. (1996). Adhesion of different Candida spp. to plastic: XTT formazan determinations. J Med Vet Mycol 34, 407-410.

Hawser, S., Francolini, M. \& Islam, K. (1996). The effects of antifungal agents on the morphogenetic transformation by Candida albicans in vitro. $J$ Antimicrob Chemother 38, 579587.

Haynes, K. (2001). Virulence in Candida species. Trends Microbio/ 9, 591-596.

Hazan, I., Sepulveda-Becerra, M. \& Liu, H. (2002). Hyphal elongation is regulated independently of cell cycle in Candida albicans. Mol Biol Cell 13, 134-145.

Heil-Chapdelaine, R. A., Oberle, J. R. \& Cooper, J. A. (2000). The cortical protein Num1p is essential for dynein-dependent interactions of microtubules with the cortex. J Cell Biol 151, $1337-1344$.

Herrero, A. B., Lopez, M. C., Fernandez-Lago, L. \& Dominguez, A. (1999a). Candida albicans and Yarrowia lipolytica as alternative models for analysing budding patterns and germ tube formation in dimorphic fungi. Microbiology 145 ( Pt 10), 2727-2737.

Herrero, A. B., Lopez, M. C., Garcia, S., Schmidt, A., Spaltmann, F., Ruiz-Herrera, J. \& Dominguez, A. (1999b). Control of filament formation in Candida albicans by polyamine levels. Infect Immun 67, 4870-4878.

Herrero, A. B., Uccelletti, D., Hirschberg, C. B., Dominguez, A. \& Abeijon, C. (2002). The Golgi GDPase of the fungal pathogen Candida albicans affects morphogenesis, glycosylation, and cell wall properties. Eukaryot Cell 1, 420-431.

Hoyer, L. L., Scherer, S., Shatzman, A. R. \& Livi, G. P. (1995). Candida albicans ALS1: domains related to a Saccharomyces cerevisiae sexual agglutinin separated by a repeating motif. Mol Microbiol 15, 39-54.

Hoyer, L. L., Payne, T. L., Bell, M., Myers, A. M. \& Scherer, S. (1998a). Candida albicans ALS3 and insights into the nature of the ALS gene family. Curr Genet 33, 451-459.

Hoyer, L. L., Payne, T. L. \& Hecht, J. E. (1998b). Identification of Candida albicans ALS2 and ALS4 and localization of als proteins to the fungal cell surface. $J$ Bacteriol 180, 5334-5343.

Hube, B. (1996). Candida albicans secreted aspartyl proteinases. Curr Top Med Mycol 7, 5569.

Hube, B. \& Naglik, J. (2001). Candida albicans proteinases: resolving the mystery of a gene family. Microbiology 147, 1997-2005.

Hull, C. M. \& Johnson, A. D. (1999). Identification of a mating type-like locus in the asexual pathogenic yeast Candida albicans. Science 285, 1271-1275.

Hull, C. M., Raisner, R. M. \& Johnson, A. D. (2000). Evidence for mating of the "asexual" yeast Candida albicans in a mammalian host. Science 289, 307-310.

Huth, K. C., Quirling, M., Maier, S. \& other authors (2009). Effectiveness of ozone against endodontopathogenic microorganisms in a root canal biofilm model. Int Endod J 42, 3-13. 
James, P., Halladay, J. \& Craig, E. A. (1996). Genomic libraries and a host strain designed for highly efficient two-hybrid selection in yeast. Genetics 144, 1425-1436.

Jansons, V. K. \& Nickerson, W. J. (1970). Chemical composition of chlamydospores of Candida albicans. J Bacteriol 104, 922-932.

Jones, T., Federspiel, N. A., Chibana, H. \& other authors (2004). The diploid genome sequence of Candida albicans. Proc Natl Acad Sci U S A 101, 7329-7334.

Kadosh, D. \& Struhl, K. (1997). Repression by Ume6 involves recruitment of a complex containing Sin3 corepressor and Rpd3 histone deacetylase to target promoters. Cell 89, 365371.

Kadosh, D. \& Johnson, A. D. (2001). Rfg1, a protein related to the Saccharomyces cerevisiae hypoxic regulator Rox1, controls filamentous growth and virulence in Candida albicans. Mol Cell Biol 21, 2496-2505.

Kadosh, D. \& Johnson, A. D. (2005). Induction of the Candida albicans filamentous growth program by relief of transcriptional repression: a genome-wide analysis. Mol Biol Cell 16, 29032912.

Kaiser, B., Munder, T., Saluz, H. P., Kunkel, W. \& Eck, R. (1999). Identification of a gene encoding the pyruvate decarboxylase gene regulator CaPdc2p from Candida albicans. Yeast $15,585-591$.

Kalo-Klein, A. \& Witkin, S. S. (1990). Prostaglandin E2 enhances and gamma interferon inhibits germ tube formation in Candida albicans. Infect Immun 58, 260-262.

Karababa, M., Coste, A. T., Rognon, B., Bille, J. \& Sanglard, D. (2004). Comparison of gene expression profiles of Candida albicans azole-resistant clinical isolates and laboratory strains exposed to drugs inducing multidrug transporters. Antimicrob Agents Chemother 48, 30643079.

Karreman, R. J. \& Lindsey, G. G. (2005). A rapid method to determine the stress status of Saccharomyces cerevisiae by monitoring the expression of a Hsp12:green fluorescent protein (GFP) construct under the control of the Hsp12 promoter. J Biomol Screen 10, 253-259.

Keleher, C. A., Redd, M. J., Schultz, J., Carlson, M. \& Johnson, A. D. (1992). Ssn6-Tup1 is a general repressor of transcription in yeast. Cell 68, 709-719.

Kelly, R., Card, D., Register, E. \& other authors (2000a). Geranylgeranyltransferase I of Candida albicans: null mutants or enzyme inhibitors produce unexpected phenotypes. $J$ Bacteriol 182, 704-713.

Kelly, T. J., Qin, S., Gottschling, D. E. \& Parthun, M. R. (2000b). Type B histone acetyltransferase Hat1p participates in telomeric silencing. Mol Cell Biol 20, 7051-7058.

Keng, T. (1992). HAP1 and ROX1 form a regulatory pathway in the repression of HEM13 transcription in Saccharomyces cerevisiae. Mol Cell Biol 12, 2616-2623.

Khalaf, R. A. \& Zitomer, R. S. (2001). The DNA binding protein Rfg1 is a repressor of filamentation in Candida albicans. Genetics 157, 1503-1512.

Kimura, A., Matsubara, K. \& Horikoshi, M. (2005). A decade of histone acetylation: marking eukaryotic chromosomes with specific codes. J Biochem (Tokyo) 138, 647-662.

Kleff, S., Andrulis, E. D., Anderson, C. W. \& Sternglanz, R. (1995). Identification of a gene encoding a yeast histone $\mathrm{H} 4$ acetyltransferase. J Biol Chem 270, 24674-24677. 
Koerkamp, M. G., Rep, M., Bussemaker, H. J., Hardy, G. P., Mul, A., Piekarska, K., Szigyarto, C. A., De Mattos, J. M. \& Tabak, H. F. (2002). Dissection of transient oxidative stress response in Saccharomyces cerevisiae by using DNA microarrays. Mol Biol Cell 13, 2783-2794.

Kohler, G. A., White, T. C. \& Agabian, N. (1997). Overexpression of a cloned IMP dehydrogenase gene of Candida albicans confers resistance to the specific inhibitor mycophenolic acid. Journal of bacteriology 179, 2331-2338.

Komachi, K. \& Johnson, A. D. (1997). Residues in the WD repeats of Tup1 required for interaction with alpha2. Mol Cell Biol 17, 6023-6028.

Kron, S. J., Styles, C. A. \& Fink, G. R. (1994). Symmetric cell division in pseudohyphae of the yeast Saccharomyces cerevisiae. Mol Biol Cell 5, 1003-1022.

Kronstad, J., De Maria, A. D., Funnell, D., Laidlaw, R. D., Lee, N., de Sa, M. M. \& Ramesh, M. (1998). Signaling via CAMP in fungi: interconnections with mitogen-activated protein kinase pathways. Arch Microbiol 170, 395-404.

Kues, U. (2000). Life history and developmental processes in the basidiomycete Coprinus cinereus. Microbiol Mol Biol Rev 64, 316-353.

Kulesh, D. A., Clive, D. R., Zarlenga, D. S. \& Greene, J. J. (1987). Identification of interferonmodulated proliferation-related cDNA sequences. Proc Natl Acad Sci U S A 84, 8453-8457.

Kuo, M. H., Brownell, J. E., Sobel, R. E., Ranalli, T. A., Cook, R. G., Edmondson, D. G., Roth, S. Y. \& Allis, C. D. (1996). Transcription-linked acetylation by Gcn5p of histones $\mathrm{H} 3$ and H4 at specific lysines. Nature 383, 269-272.

Kuo, M. H. \& Allis, C. D. (1998). Roles of histone acetyltransferases and deacetylases in gene regulation. Bioessays 20, 615-626.

Kuo, M. H., Zhou, J., Jambeck, P., Churchill, M. E. \& Allis, C. D. (1998). Histone acetyltransferase activity of yeast Gcn5p is required for the activation of target genes in vivo. Genes Dev 12, 627-639.

Kuo, M. H., Brownell, J. E., Sobel, R. E., Ranalli, T. A., Cook, R. G., Edmondson, D. G., Roth, \& S. Y. y Allis, C. D. (1996). Transcription-linked acetylation by Gcn5p of histones H3 and $\mathrm{H} 4$ at specific lysines. Nature $383,269-272$.

Kurdistani, S. K. \& Grunstein, M. (2003). Histone acetylation and deacetylation in yeast. Nat Rev Mol Cell Biol 4, 276-284.

Kurtz, M. B., Kirsch, D. R. \& Kelly, R. (1988). The molecular genetics of Candida albicans. Microbiol Sci 5, 58-63.

Kurzai, O., Schmitt, C., Brocker, E., Frosch, M. \& Kolb-Maurer, A. (2005). Polymorphism of Candida albicans is a major factor in the interaction with human dendritic cells. Int $J$ Med Microbiol 295, 121-127.

Lagorce, A., Hauser, N. C., Labourdette, D., Rodriguez, C., Martin-Yken, H., Arroyo, J., Hoheisel, J. D. \& Francois, J. (2003). Genome-wide analysis of the response to cell wall mutations in the yeast Saccharomyces cerevisiae. J Biol Chem 278, 20345-20357.

Lan, C. Y., Newport, G., Murillo, L. A., Jones, T., Scherer, S., Davis, R. W. \& Agabian, N. (2002). Metabolic specialization associated with phenotypic switching in Candidaalbicans. Proc Natl Acad Sci U S A 99, 14907-14912. 
Lan, C. Y., Rodarte, G., Murillo, L. A., Jones, T., Davis, R. W., Dungan, J., Newport, G. \& Agabian, N. (2004). Regulatory networks affected by iron availability in Candida albicans. Mol Microbiol 53, 1451-1469.

Lane, S., Birse, C., Zhou, S., Matson, R. \& Liu, H. (2001). DNA array studies demonstrate convergent regulation of virulence factors by Cph1, Cph2, and Efg1 in Candida albicans. J Biol Chem 276, 48988-48996.

Lashkari, D. A., DeRisi, J. L., McCusker, J. H., Namath, A. F., Gentile, C., Hwang, S. Y., Brown, P. O. \& Davis, R. W. (1997). Yeast microarrays for genome wide parallel genetic and gene expression analysis. Proc Natl Acad Sci U S A 94, 13057-13062.

Leberer, E., Harcus, D., Broadbent, I. D. \& other authors (1996). Signal transduction through homologs of the Ste20p and Ste7p protein kinases can trigger hyphal formation in the pathogenic fungus Candida albicans. Proc Natl Acad Sci U S A 93, 13217-13222.

Lee, K. K. \& Workman, J. L. (2007). Histone acetyltransferase complexes: one size doesn't fit all. Nat Rev Mol Cell Biol 8, 284-295.

Lee, K. L., Buckley, H. R. \& Campbell, C. C. (1975a). An amino acid liquid synthetic medium for the development of mycelial and yeast forms of Candida Albicans. Sabouraudia 13, 148153.

Lee, K. L., Rega, M. E. \& Campbell, C. C. (1975b). Amino acid synthetic media for fungal pathogens based on aminopeptidase specificities: Histoplasma capsulatum, Blastomyces dermititidis, Paracoccidioides brasiliensis and Cryptococcus neoformans. Sabouraudia 13, 142147.

Leng, P., Carter, P. E. \& Brown, A. J. (1998). The TATA-binding protein (TBP) from the human fungal pathogen Candida albicans can complement defects in human and yeast TBPs. $J$ Bacteriol 180, 1771-1776.

Leng, P., Sudbery, P. E. \& Brown, A. J. (2000). Rad6p represses yeast-hypha morphogenesis in the human fungal pathogen Candida albicans. Mol Microbiol 35, 1264-1275.

Lengeler K.B., D. R. C., D Souza C., Arracima T., Shen W.-C., Wang P., Pan X., Waugh M. and Heitman J. (2000). Signal transduction cascades regulating fangal development and virulence. Microbiol Mol Biol Rev 64, 746-785.

Leuker, C. E., Sonneborn, A., Delbruck, S. \& Ernst, J. F. (1997). Sequence and promoter regulation of the PCK1 gene encoding phosphoenolpyruvate carboxykinase of the fungal pathogen Candida albicans. Gene 192, 235-240.

Levin-Salomon, V., Maayan, I., Avrahami-Moyal, L., Marbach, I., Livnah, O. \& Engelberg, D. (2009). When expressed in yeast, mammalian mitogen-activated protein kinases lose proper regulation and become spontaneously phosphorylated. Biochem J 417, 331-340.

Levitz, S. M. \& North, E. A. (1996). gamma Interferon gene expression and release in human lymphocytes directly activated by Cryptococcus neoformans and Candida albicans. Infect Immun 64, 1595-1599.

Li, J. J. \& Kelly, T. J. (1984). Simian virus 40 DNA replication in vitro. Proc Natl Acad Sci U S A 81, 6973-6977.

Liu, H., Kohler, J. \& Fink, G. R. (1994). Suppression of hyphal formation in Candida albicans by mutation of a STE12 homolog. Science 266, 1723-1726.

Liu, H. (2001). Transcriptional control of dimorphism in Candida albicans. Curr Opin Microbiol 4, 728-735. 
Liu, T. T., Lee, R. E., Barker, K. S., Lee, R. E., Wei, L., Homayouni, R. \& Rogers, P. D. (2005). Genome-wide expression profiling of the response to azole, polyene, echinocandin, and pyrimidine antifungal agents in Candida albicans. Antimicrob Agents Chemother 49, 2226-2236.

Lo, H. J., Kohler, J. R., DiDomenico, B., Loebenberg, D., Cacciapuoti, A. \& Fink, G. R. (1997). Nonfilamentous C. albicans mutants are avirulent. Cell 90, 939-949.

Loeb, J. D., Kerentseva, T. A., Pan, T., Sepulveda-Becerra, M. \& Liu, H. (1999a). Saccharomyces cerevisiae G1 cyclins are differentially involved in invasive and pseudohyphal growth independent of the filamentation mitogen-activated protein kinase pathway. Genetics $153,1535-1546$.

Loeb, J. D., Sepulveda-Becerra, M., Hazan, I. \& Liu, H. (1999b). A G1 cyclin is necessary for maintenance of filamentous growth in Candida albicans. Mol Cell Biol 19, 4019-4027.

Lorenz, M. C., Bender, J. A. \& Fink, G. R. (2004). Transcriptional response of Candida albicans upon internalization by macrophages. Eukaryot Cell 3, 1076-1087.

Luger, K., Mader, A. W., Richmond, R. K., Sargent, D. F. \& Richmond, T. J. (1997). Crystal structure of the nucleosome core particle at 2.8 A resolution. Nature $\mathbf{3 8 9}, 251-260$.

Lusser, A., Eberharter, A., Loidl, A., Goralik-Schramel, M., Horngacher, M., Haas, H. \& Loidl, P. (1999). Analysis of the histone acetyltransferase B complex of maize embryos. Nucleic Acids Res 27, 4427-4435.

Lusser, A., Kolle, D. \& Loidl, P. (2001). Histone acetylation: lessons from the plant kingdom. Trends Plant Sci 6, 59-65.

Lussier, M., White,A.M., Sheraton,J., di Paolo,T., Treadwell,J., Southard,S.B., Horenstein,C.I., Chen-Weiner,J., and et al., (1997). Large scale identification of genes involved in cell surface biosynthesis and architecture in Saccharomyces cerevisiae. Genetics $147,435-450$.

Magee, B. B. \& Magee, P. T. (2000). Induction of mating in Candida albicans by construction of MTLa and MTLalpha strains. Science 289, 310-313.

Magee, B. B., Legrand, M., Alarco, A. M., Raymond, M. \& Magee, P. T. (2002). Many of the genes required for mating in Saccharomyces cerevisiae are also required for mating in Candida albicans. Mol Microbiol 46, 1345-1351.

Magee, P. T. \& Magee, B. B. (2004). Through a glass opaquely: the biological significance of mating in Candida albicans. Curr Opin Microbiol 7, 661-665.

Maglott, D., Ostell, J., Pruitt, K. D. \& Tatusova, T. (2007). Entrez Gene: gene-centered information at NCBI. Nucleic Acids Res 35, D26-31.

Maicas, S., Moreno, I., Nieto, A., Gomez, M., Sentandreu, R. \& Valentin, E. (2005). In Silico Analysis for Transcription Factors With Zn(II)(2)C(6) Binuclear Cluster DNA-Binding Domains in Candida albicans. Comparative and functional genomics 6, 345-356.

Maidan, M. M., De Rop, L., Serneels, J., Exler, S., Rupp, S., Tournu, H., Thevelein, J. M. \& Van Dijck, P. (2005). The G protein-coupled receptor Gpr1 and the Galpha protein Gpa2 act through the cAMP-protein kinase A pathway to induce morphogenesis in Candida albicans. Mol Biol Cell 16, 1971-1986.

Maidan, M. M., De Rop, L., Relloso, M., Diez-Orejas, R., Thevelein, J. M. \& Van Dijck, P. (2008). Combined inactivation of the Candida albicans GPR1 and TPS2 genes results in avirulence in a mouse model for systemic infection. Infect Immun 76, 1686-1694. 
Makowski, A. M., Dutnall, R. N. \& Annunziato, A. T. (2001). Effects of acetylation of histone $\mathrm{H} 4$ at lysines 8 and 16 on activity of the Hat1 histone acetyltransferase. J Biol Chem 276, 43499-43502.

Maneu, V., Cervera, A. M., Martinez, J. P. \& Gozalbo, D. (1996). Molecular cloning and characterization of a Candida albicans gene (EFB1) coding for the elongation factor EF-1 beta. FEMS Microbiol Lett 145, 157-162.

Marchais, V., Kempf, M., Licznar, P., Lefrancois, C., Bouchara, J. P., Robert, R. \& Cottin, J. (2005). DNA array analysis of Candida albicans gene expression in response to adherence to polystyrene. FEMS Microbiol Lett 245, 25-32.

Marichal, P., Gorrens, J., Laurijssens, L. \& other authors (1999). Accumulation of 3ketosteroids induced by itraconazole in azole-resistant clinical Candida albicans isolates. Antimicrob Agents Chemother 43, 2663-2670.

Martchenko, M., Levitin, A., Hogues, H., Nantel, A. \& Whiteway, M. (2007). Transcriptional rewiring of fungal galactose-metabolism circuitry. Curr Biol 17, 1007-1013.

Martin, S. W., Douglas, L. M. \& Konopka, J. B. (2005). Cell cycle dynamics and quorum sensing in Candida albicans chlamydospores are distinct from budding and hyphal growth. Eukaryot Cell 4, 1191-1202.

Martinez-Lopez, R., Nombela, C., Diez-Orejas, R., Monteoliva, L. \& Gil, C. (2008). Immunoproteomic analysis of the protective response obtained from vaccination with Candida albicans ecm33 cell wall mutant in mice. Proteomics 8, 2651-2664.

Martinez, J. P., Lopez-Ribot, J. L., Gil, M. L., Sentandreu, R. \& Ruiz-Herrera, J. (1990). Inhibition of the dimorphic transition of Candida albicans by the ornithine decarboxylase inhibitor 1,4-diaminobutanone: alterations in the glycoprotein composition of the cell wall. $J$ Gen Microbiol 136, 1937-1943.

Martinez Roig, A. (2003). Micosis cutáneas. In Protocolos diagnósticos y terapeúticos en dermatología pediátrica, pp. 65-73. Edited by F. A. M. Llop: Asociación Española de Pediatría.

Martins-Nishikawa, M., Trilles, L., Symoens, F., Swinne, D. \& Nolard, N. (2002). Prevalence of Candida dubliniensis in the BCCM/IHEM Biomedical Fungi/Yeasts culture collection (isolates before 1990). Med Mycol 40, 443-445.

McAlister, L. \& Holland, M. J. (1985). Isolation and characterization of yeast strains carrying mutations in the glyceraldehyde-3-phosphate dehydrogenase genes. The Journal of biological chemistry 260, 15013-15018.

McCreath, K. J., Specht, C. A. \& Robbins, P. W. (1995). Molecular cloning and characterization of chitinase genes from Candida albicans. Proc Natl Acad Sci U S A 92, 25442548.

McCreath, K. J., Specht, C. A., Liu, Y. \& Robbins, P. W. (1996). Molecular cloning of a third chitinase gene (CHT1) from Candida albicans. Yeast 12, 501-504.

Melo, N. R., Moran, G. P., Warrilow, A. G. \& other authors (2008). CYP56 (Dit2p) in Candida albicans: characterization and investigation of its role in growth and antifungal drug susceptibility. Antimicrob Agents Chemother 52, 3718-3724.

Mio, T., Yabe, T., Sudoh, M., Satoh, Y., Nakajima, T., Arisawa, M. \& Yamada-Okabe, H. (1996). Role of three chitin synthase genes in the growth of Candida albicans. J Bacteriol 178, 2416-2419. 
Mio, T., Adachi-Shimizu, M., Tachibana, Y. \& other authors (1997). Cloning of the Candida albicans homolog of Saccharomyces cerevisiae GSC1/FKS1 and its involvement in beta-1,3glucan synthesis. J Bacteriol 179, 4096-4105.

Mirbod, F., Nakashima, S., Kitajima, Y., Ghannoum, M. A., Cannon, R. D. \& Nozawa, Y. (1996). Molecular cloning of a gene encoding translation initiation factor (TIF) from Candida albicans. J Med Vet Mycol 34, 393-400.

Mirbod, F., Nakashima, S., Kitajima, Y., Cannon, R. D. \& Nozawa, Y. (1997). Molecular cloning of a Rho family, CDC42Ca gene from Candida albicans and its mRNA expression changes during morphogenesis. J Med Vet Mycol 35, 173-179.

Mitrovich, Q. M., Tuch, B. B., Guthrie, C. \& Johnson, A. D. (2007). Computational and experimental approaches double the number of known introns in the pathogenic yeast Candida albicans. Genome Res 17, 492-502.

Miwa, T., Takagi, Y., Shinozaki, M., Yun, C. W., Schell, W. A., Perfect, J. R., Kumagai, H. \& Tamaki, H. (2004). Gpr1, a putative G-protein-coupled receptor, regulates morphogenesis and hypha formation in the pathogenic fungus Candida albicans. Eukaryot Cell 3, 919-931.

Molero, G., Diez-Orejas, R., Navarro-Garcia, F., Monteoliva, L., Pla, J., Gil, C., SanchezPerez, M. \& Nombela, C. (1998). Candida albicans: genetics, dimorphism and pathogenicity. Int Microbiol 1, 95-106.

Mondesert, G., Clarke, D. J. \& Reed, S. I. (1997). Identification of genes controlling growth polarity in the budding yeast Saccharomyces cerevisiae: a possible role of $\mathrm{N}$-glycosylation and involvement of the exocyst complex. Genetics 147, 421-434.

Monterola, F. (2002).Tesis Doctoral: Dimorfismo en Yarrowia lipolytica y Candida albicans. Aislamiento del gen SIN3, un factor de regulación transcripcional. In Departamento de Microbiología y Genética. Salamanca: Universidad de Salamanca.

Mora-Montes, H. M., Bates, S., Netea, M. G. \& other authors (2007). Endoplasmic reticulum alpha-glycosidases of Candida albicans are required for $\mathrm{N}$ glycosylation, cell wall integrity, and normal host-fungus interaction. Eukaryot Cell 6, 2184-2193.

Moreno, I., Castillo, L., Sentandreu, R. \& Valentin, E. (2007). Global transcriptional profiling of Candida albicans cwt1 null mutant. Yeast 24, 357-370.

Mori, K., Kawahara, T., Yoshida, H., Yanagi, H. \& Yura, T. (1996). Signalling from endoplasmic reticulum to nucleus: transcription factor with a basic-leucine zipper motif is required for the unfolded protein-response pathway. Genes Cells 1, 803-817.

Morschhauser, J., Staib, P. \& Kohler, G. (2005). Targeted gene deletion in Candida albicans wild-type strains by MPAR flipping. Methods Mol Med 118, 35-44.

Morschhauser, J., Barker, K. S., Liu, T. T., Bla, B. W. J., Homayouni, R. \& Rogers, P. D. (2007). The transcription factor Mrr1p controls expression of the MDR1 efflux pump and mediates multidrug resistance in Candida albicans. PLoS Pathog 3, e164.

Muller, E. H., Richards, E. J., Norbeck, J., Byrne, K. L., Karlsson, K. A., Pretorius, G. H., Meacock, P. A., Blomberg, A. \& Hohmann, S. (1999). Thiamine repression and pyruvate decarboxylase autoregulation independently control the expression of the Saccharomyces cerevisiae PDC5 gene. FEBS Lett 449, 245-250.

Munro, C. A., Schofield, D. A., Gooday, G. W. \& Gow, N. A. (1998). Regulation of chitin synthesis during dimorphic growth of Candida albicans. Microbiology 144 ( Pt 2), 391-401. 
Munro, C. A., Bates, S., Buurman, E. T. \& other authors (2005). Mnt1p and Mnt $2 p$ of Candida albicans are partially redundant alpha-1,2-mannosyltransferases that participate in Olinked mannosylation and are required for adhesion and virulence. J Biol Chem 280, 1051-1060.

Murad, A. M., d'Enfert, C., Gaillardin, C. \& other authors (2001a). Transcript profiling in Candida albicans reveals new cellular functions for the transcriptional repressors CaTup1, CaMig1 and CaNrg1. Mol Microbiol 42, 981-993.

Murad, A. M., Leng, P., Straffon, M. \& other authors (2001b). NRG1 represses yeast-hypha morphogenesis and hypha-specific gene expression in Candida albicans. Embo J 20, 47424752.

Murillo, L. A., Newport, G., Lan, C. Y., Habelitz, S., Dungan, J. \& Agabian, N. M. (2005). Genome-wide transcription profiling of the early phase of biofilm formation by Candida albicans. Eukaryot Cell 4, 1562-1573.

Murray, B., Alessandrini, A., Cole, A. J., Yee, A. G. \& Furshpan, E. J. (1998). Inhibition of the p44/42 MAP kinase pathway protects hippocampal neurons in a cell-culture model of seizure activity. Proc Natl Acad Sci U S A 95, 11975-11980.

Myers, K. K., Fonzi, W. A. \& Sypherd, P. S. (1992). Isolation and sequence analysis of the gene for translation elongation factor 3 from Candida albicans. Nucleic Acids Res 20, 17051710.

Naglik, J. R., Challacombe, S. J. \& Hube, B. (2003). Candida albicans secreted aspartyl proteinases in virulence and pathogenesis. Microbiol Mol Biol Rev 67, 400-428, table of contents.

Nantel, A., Dignard, D., Bachewich, C. \& other authors (2002). Transcription profiling of Candida albicans cells undergoing the yeast-to-hyphal transition. Mol Biol Cell 13, 3452-3465.

Natarajan, K., Meyer, M. R., Jackson, B. M., Slade, D., Roberts, C., Hinnebusch, A. G. \& Marton, M. J. (2001). Transcriptional profiling shows that Gcn4p is a master regulator of gene expression during amino acid starvation in yeast. Mol Cell Biol 21, 4347-4368.

Nehlin, J. O., Carlberg, M. \& Ronne, H. (1992). Yeast SKO1 gene encodes a bZIP protein that binds to the CRE motif and acts as a repressor of transcription. Nucleic Acids Res 20, 52715278.

Newport, M. J. \& Nejentsev, S. (2004). Genetics of susceptibility to tuberculosis in humans. Monaldi Arch Chest Dis 61, 102-111.

Niewerth, M., Kunze, D., Seibold, M., Schaller, M., Korting, H. C. \& Hube, B. (2003). Ciclopirox olamine treatment affects the expression pattern of Candida albicans genes encoding virulence factors, iron metabolism proteins, and drug resistance factors. Antimicrob Agents Chemother 47, 1805-1817.

Niu, W., Li, Z., Zhan, W., lyer, V. R. \& Marcotte, E. M. (2008). Mechanisms of cell cycle control revealed by a systematic and quantitative overexpression screen in S. cerevisiae. PLoS Genet 4, e1000120.

Nobile, C. J., Bruno, V. M., Richard, M. L., Davis, D. A. \& Mitchell, A. P. (2003). Genetic control of chlamydospore formation in Candida albicans. Microbiology 149, 3629-3637.

Nobile, C. J. \& Mitchell, A. P. (2005). Regulation of cell-surface genes and biofilm formation by the C. albicans transcription factor Bcr1p. Curr Biol 15, 1150-1155.

Noble, S. M. \& Johnson, A. D. (2005). Strains and strategies for large-scale gene deletion studies of the diploid human fungal pathogen Candida albicans. Eukaryot Cell 4, 298-309. 
Noffz, C. S., Liedschulte, V., Lengeler, K. \& Ernst, J. F. (2008). Functional mapping of the Candida albicans Efg1 regulator. Eukaryot Cell 7, 881-893.

Nojima, H., Leem, S. H., Araki, H., Sakai, A., Nakashima, N., Kanaoka, Y. \& Ono, Y. (1994). Hac1: a novel yeast bZIP protein binding to the CRE motif is a multicopy suppressor for cdc10 mutant of Schizosaccharomyces pombe. Nucleic Acids Res 22, 5279-5288.

Nordle, A. K., Rios, P., Gaulton, A., Pulido, R., Attwood, T. K. \& Tabernero, L. (2007). Functional assignment of MAPK phosphatase domains. Proteins 69, 19-31.

O'Rourke, S. M., Herskowitz, I. \& O'Shea, E. K. (2002). Yeast go the whole HOG for the hyperosmotic response. Trends Genet 18, 405-412.

Odds, F. C., Webster, C. E., Riley, V. C. \& Fisk, P. G. (1987). Epidemiology of vaginal Candida infection: significance of numbers of vaginal yeasts and their biotypes. Eur $\mathrm{J}$ Obstet Gynecol Reprod Biol 25, 53-66.

Odds, F. C. (1988). Candida and candidosis. London: Balliere-Tindall.

Odds, F. C., Webster, C. E., Mayuranathan, P. \& Simmons, P. D. (1988). Candida concentrations in the vagina and their association with signs and symptoms of vaginal candidosis. J Med Vet Mycol 26, 277-283.

Odds, F. C., Van Nuffel, L. \& Dams, G. (1998). Prevalence of Candida dubliniensis isolates in a yeast stock collection. J Clin Microbiol 36, 2869-2873.

Odds, F. C., Brown, A. J. \& Gow, N. A. (2004). Candida albicans genome sequence: a platform for genomics in the absence of genetics. Genome Biol 5, 230.

Opilla, M. (2008). Epidemiology of bloodstream infection associated with parenteral nutrition. Am J Infect Control 36, S173 e175-178.

Overkamp, K. M., Bakker, B. M., Kotter, P., Luttik, M. A., Van Dijken, J. P. \& Pronk, J. T. (2002). Metabolic engineering of glycerol production in Saccharomyces cerevisiae. Appl Environ Microbio/ 68, 2814-2821.

Paraje, M. G., Correa, S. G., Renna, M. S., Theumer, M. \& Sotomayor, C. E. (2008). Candida albicans-secreted lipase induces injury and steatosis in immune and parenchymal cells. Can $J$ Microbiol 54, 647-659.

Park, J. Y., Kim, D. H., Shin, W. S., Seo, H. S., Kim, K., Lee, K. H., Koh, C. M. \& Bong, J. P. (2003). Search for genes potentially related to germ tube formation in Candida albicans by differential-display reverse transcription polymerase chain reaction. Yonsei medical journal 44, 110-118.

Park, Y. N. \& Morschhauser, J. (2005). Tetracycline-inducible gene expression and gene deletion in Candida albicans. Eukaryot Cell 4, 1328-1342.

Parthun, M. R., Widom, J. \& Gottschling, D. E. (1996). The major cytoplasmic histone acetyltransferase in yeast: links to chromatin replication and histone metabolism. Cell 87, 85-94.

Parthun, M. R. (2007). Hat1: the emerging cellular roles of a type B histone acetyltransferase. Oncogene 26, 5319-5328.

Peman, J., Canton, E. \& Gobernado, M. (2005). Epidemiology and antifungal susceptibility of Candida species isolated from blood: results of a 2-year multicentre study in Spain. Eur J Clin Microbiol Infect Dis 24, 23-30. 
Penn, M. D., Galgoci, B. \& Greer, H. (1983). Identification of AAS genes and their regulatory role in general control of amino acid biosynthesis in yeast. Proc Natl Acad Sci U S A 80, 27042708.

Pereira-Cenci, T., Del Bel Cury, A. A., Crielaard, W. \& Ten Cate, J. M. (2008). Development of Candida-associated denture stomatitis: new insights. J Appl Oral Sci 16, 86-94.

Pile, L. A., Schlag, E. M. \& Wassarman, D. A. (2002). The SIN3/RPD3 deacetylase complex is essential for $\mathrm{G}(2)$ phase cell cycle progression and regulation of SMRTER corepressor levels. Mol Cell Biol 22, 4965-4976.

Pile, L. A., Spellman, P. T., Katzenberger, R. J. \& Wassarman, D. A. (2003). The SIN3 deacetylase complex represses genes encoding mitochondrial proteins: implications for the regulation of energy metabolism. J Biol Chem 278, 37840-37848.

Pnueli, L., Edry, I., Cohen, M. \& Kassir, Y. (2004). Glucose and nitrogen regulate the switch from histone deacetylation to acetylation for expression of early meiosis-specific genes in budding yeast. Mol Cell Biol 24, 5197-5208.

Poveda, A., Pamblanco, M., Tafrov, S., Tordera, V., Sternglanz, R. \& Sendra, R. (2004). Hif1 is a component of yeast histone acetyltransferase $B$, a complex mainly localized in the nucleus. The Journal of biological chemistry 279, 16033-16043.

Poveda, A. \& Sendra, R. (2008). Site specificity of yeast histone acetyltransferase B complex in vivo. The FEBS journal 275, 2122-2136.

Prigneau, O., Porta, A., Poudrier, J. A., Colonna-Romano, S., Noel, T. \& Maresca, B. (2003). Genes involved in beta-oxidation, energy metabolism and glyoxylate cycle are induced by Candida albicans during macrophage infection. Yeast 20, 723-730.

Prill, S. K., Klinkert, B., Timpel, C., Gale, C. A., Schroppel, K. \& Ernst, J. F. (2005). PMT family of Candida albicans: five protein mannosyltransferase isoforms affect growth, morphogenesis and antifungal resistance. Mol Microbiol 55, 546-560.

Proft, M., Gibbons, F. D., Copeland, M., Roth, F. P. \& Struhl, K. (2005). Genomewide identification of Sko1 target promoters reveals a regulatory network that operates in response to osmotic stress in Saccharomyces cerevisiae. Eukaryot Cell 4, 1343-1352.

Qin, S. \& Parthun, M. R. (2002). Histone $\mathrm{H} 3$ and the histone acetyltransferase Hat $1 \mathrm{p}$ contribute to DNA double-strand break repair. Mol Cell Biol 22, 8353-8365.

Ram, A. F., Wolters, A., Ten Hoopen, R. \& Klis, F. M. (1994). A new approach for isolating cell wall mutants in Saccharomyces cerevisiae by screening for hypersensitivity to calcofluor white. Yeast 10, 1019-1030.

Ramsdale, M., Selway, L., Stead, D., Walker, J., Yin, Z., Nicholls, S. M., Crowe, J., Sheils, E. M. \& Brown, A. J. (2008). MNL1 regulates weak acid-induced stress responses of the fungal pathogen Candida albicans. Mol Biol Cell 19, 4393-4403.

Rashki (2009).Transcripcional analysis of the histone acetyltation and deacetylation in Candida albicans. In Microbiology and Geneticss. Salamanca: Salamanca.

Rasmussen, S. W. (1995). A $37.5 \mathrm{~kb}$ region of yeast chromosome $X$ includes the SME1, MEF2, GSH1 and CSD3 genes, a TCP-1-related gene, an open reading frame similar to the DAL80 gene, and a tRNA(Arg). Yeast 11, 873-883.

Reifenberger, E., Freidel, K. \& Ciriacy, M. (1995). Identification of novel HXT genes in Saccharomyces cerevisiae reveals the impact of individual hexose transporters on glycolytic flux. Mol Microbiol 16, 157-167. 
Reuss, O., Vik, A., Kolter, R. \& Morschhauser, J. (2004). The SAT1 flipper, an optimized tool for gene disruption in Candida albicans. Gene 341, 119-127.

Reuss, O. \& Morschhauser, J. (2006). A family of oligopeptide transporters is required for growth of Candida albicans on proteins. Mol Microbiol 60, 795-812.

Robyr, D., Suka, Y., Xenarios, I., Kurdistani, S. K., Wang, A., Suka, N. \& Grunstein, M. (2002). Microarray deacetylation maps determine genome-wide functions for yeast histone deacetylases. Cell 109, 437-446.

Rogers, P. D. \& Barker, K. S. (2003). Genome-wide expression profile analysis reveals coordinately regulated genes associated with stepwise acquisition of azole resistance in Candida albicans clinical isolates. Antimicrob Agents Chemother 47, 1220-1227.

Roman, E., Arana, D. M., Nombela, C., Alonso-Monge, R. \& Pla, J. (2007). MAP kinase pathways as regulators of fungal virulence. Trends Microbiol 15, 181-190.

Rosaleny, L. E., Antunez, O., Ruiz-Garcia, A. B., Perez-Ortin, J. E. \& Tordera, V. (2005). Yeast HAT1 and HAT2 deletions have different life-span and transcriptome phenotypes. FEBS Lett 579, 4063-4068.

Rose, M. D. W., F; Hieter, P (1990).Methods in Yeast Genetics : A Laboratory Manual: Plainview, NY: Cold Spring Harbor Lab. Press.

Rossignol, T., Lechat, P., Cuomo, C., Zeng, Q., Moszer, I. \& d'Enfert, C. (2008). CandidaDB: a multi-genome database for Candida species and related Saccharomycotina. Nucleic Acids Res 36, D557-561.

Rottmann, M., Dieter, S., Brunner, H. \& Rupp, S. (2003). A screen in Saccharomyces cerevisiae identified CaMCM1, an essential gene in Candida albicans crucial for morphogenesis. Mol Microbiol 47, 943-959.

Ruiz-Garcia, A. B., Sendra, R., Galiana, M., Pamblanco, M., Perez-Ortin, J. E. \& Tordera, V. (1998). HAT1 and HAT2 proteins are components of a yeast nuclear histone acetyltransferase enzyme specific for free histone H4. J Biol Chem 273, 12599-12605.

Ruiz, C. (2004).Resumenes ICCAAC. Epidemiología y clínica de la infección fúngica. In Sistema interactivo de información en enfermedades infecciosas y microbiología médica. Edited by D. J. L. Rodríguez-Tudela: ICCAAC.

Ruiz, T., Sanchez, M., De la Rosa, J. M., Rodriguez, L. \& Dominguez, A. (2001). The sequence of a 15769 bp segment of Pichia anomala identifies the SEC61 and FBP1 genes and five new open reading frames. Yeast 18, 1187-1195.

Rundlett, S. E., Carmen, A. A., Suka, N., Turner, B. M. \& Grunstein, M. (1998). Transcriptional repression by UME6 involves deacetylation of lysine 5 of histone H4 by RPD3. Nature 392, 831-835.

Sabie, F. T. \& Gadd, G. M. (1992). Effect of nucleosides and nucleotides and the relationship between cellular adenosine 3':5'-cyclic monophosphate (cyclic AMP) and germ tube formation in Candida albicans. Mycopathologia 119, 147-156.

Sambrook, J., David, W., Russsell (2001). Molecular cloning: a laboratory manual,: Cold Spring Harbor Laboratory, Cold Spring Harbor, N.Y.

Sanchez-Martinez, C. \& Perez-Martin, J. (2001). Dimorphism in fungal pathogens: Candida albicans and Ustilago maydis--similar inputs, different outputs. Curr Opin Microbiol 4, 214-221. 
Sanchez-Martinez, C. \& Perez-Martin, J. (2002). Gpa2, a G-protein alpha subunit required for hyphal development in Candida albicans. Eukaryot Cell 1, 865-874.

Saville, S. P., Lazzell, A. L., Monteagudo, C. \& Lopez-Ribot, J. L. (2003). Engineered control of cell morphology in vivo reveals distinct roles for yeast and filamentous forms of Candida albicans during infection. Eukaryot Cell 2, 1053-1060.

Schatz, P. J., Solomon, F. \& Botstein, D. (1988). Isolation and characterization of conditionallethal mutations in the TUB1 alpha-tubulin gene of the yeast Saccharomyces cerevisiae. Genetics 120, 681-695.

Schena, M., Shalon, D., Davis, R. W. \& Brown, P. O. (1995). Quantitative monitoring of gene expression patterns with a complementary DNA microarray. Science 270, 467-470.

Seneviratne, C. J., Wang, Y., Jin, L., Abiko, Y. \& Samaranayake, L. P. (2008). Candida albicans biofilm formation is associated with increased anti-oxidative capacities. Proteomics $\mathbf{8}$, 2936-2947.

Sharkey, L. L., Liao, W. L., Ghosh, A. K. \& Fonzi, W. A. (2005). Flanking direct repeats of hisG alter URA3 marker expression at the HWP1 locus of Candida albicans. Microbiology 151, 1061-1071.

Shaw, J. R., Colbourne, J. K., Davey, J. C., Glaholt, S. P., Hampton, T. H., Chen, C. Y., Folt, C. L. \& Hamilton, J. W. (2007). Gene response profiles for Daphnia pulex exposed to the environmental stressor cadmium reveals novel crustacean metallothioneins. BMC Genomics 8 , 477.

Shen, J., Guo, W. \& Kohler, J. R. (2005). CaNAT1, a heterologous dominant selectable marker for transformation of Candida albicans and other pathogenic Candida species. Infect Immun 73, 1239-1242.

Shepherd, M. G., Yin, C. Y., Ram, S. P. \& Sullivan, P. A. (1980). Germ tube induction in Candida albicans. Can J Microbiol 26, 21-26.

Sherman, F., G. R. Fink, and J. B. Hicks. (1986). Methods in yeast genetics: Cold Spring Harbor Laboratories.

Silva, V., Cabrera, M., Diaz, M. C., Abarca, C. \& Hermosilla, G. (2003). [Prevalence of Candida albican serotypes in blood isolates in Chile, and first report of Candida dubliniensis candidemia]. Rev Iberoam Micol 20, 46-51.

Silverstein, R. A. \& Ekwall, K. (2005). Sin3: a flexible regulator of global gene expression and genome stability. Curr Genet 47, 1-17.

Singh, V., Sinha, I. \& Sadhale, P. P. (2005). Global analysis of altered gene expression during morphogenesis of Candida albicans in vitro. Biochem Biophys Res Commun 334, 1149-1158.

Smith, R. L. \& Johnson, A. D. (2000). Turning genes off by Ssn6-Tup1: a conserved system of transcriptional repression in eukaryotes. Trends Biochem Sci 25, 325-330.

Soares, B. M., da Silva, D. L., Sousa, G. R., Amorim, J. C., de Resende, M. A., Pinotti, M. \& Cisalpino, P. S. (2008). In vitro photodynamic inactivation of Candida spp. growth and adhesion to buccal epithelial cells. J Photochem Photobiol B.

Sohn, K., Urban, C., Brunner, H. \& Rupp, S. (2003). EFG1 is a major regulator of cell wall dynamics in Candida albicans as revealed by DNA microarrays. Mol Microbiol 47, 89-102.

Soll, D. R., Lockhart, S. R. \& Zhao, R. (2003). Relationship between switching and mating in Candida albicans. Eukaryot Cell 2, 390-397. 
Sonneborn, A., Bockmuhl, D. P. \& Ernst, J. F. (1999a). Chlamydospore formation in Candida albicans requires the Efg1p morphogenetic regulator. Infect Immun 67, 5514-5517.

Sonneborn, A., Tebarth, B. \& Ernst, J. F. (1999b). Control of white-opaque phenotypic switching in Candida albicans by the Efg1p morphogenetic regulator. Infect Immun 67, 46554660 .

Sonneborn, A., Bockmuhl, D. P., Gerads, M., Kurpanek, K., Sanglard, D. \& Ernst, J. F. (2000). Protein kinase A encoded by TPK2 regulates dimorphism of Candida albicans. Mol Microbiol 35, 386-396.

Spector, M. S., Raff, A., DeSilva, H., Lee, K. \& Osley, M. A. (1997). Hir1p and Hir2p function as transcriptional corepressors to regulate histone gene transcription in the Saccharomyces cerevisiae cell cycle. Mol Cell Biol 17, 545-552.

Staab, J. F., Bradway, S. D., Fidel, P. L. \& Sundstrom, P. (1999). Adhesive and mammalian transglutaminase substrate properties of Candida albicans Hwp1. Science 283, 1535-1538.

Staab, J. F., Bahn, Y. S. \& Sundstrom, P. (2003). Integrative, multifunctional plasmids for hypha-specific or constitutive expression of green fluorescent protein in Candida albicans. Microbiology 149, 2977-2986.

Staebell, M. \& Soll, D. R. (1985). Temporal and spatial differences in cell wall expansion during bud and mycelium formation in Candida albicans. J Gen Microbiol 131, 1467-1480.

Sterner, D. E. \& Berger, S. L. (2000). Acetylation of histones and transcription-related factors. Microbiol Mol Biol Rev 64, 435-459.

Stoldt, V. R., Sonneborn, A., Leuker, C. E. \& Ernst, J. F. (1997). Efg1p, an essential regulator of morphogenesis of the human pathogen Candida albicans, is a member of a conserved class of bHLH proteins regulating morphogenetic processes in fungi. Embo J 16, 1982-1991.

Sudbery, P., Gow, N. \& Berman, J. (2004). The distinct morphogenic states of Candida albicans. Trends Microbiol 12, 317-324.

Sudbery, P. E. (2001). The germ tubes of Candida albicans hyphae and pseudohyphae show different patterns of septin ring localization. Mol Microbiol 41, 19-31.

Sullivan, D. J., Moran, G. P. \& Coleman, D. C. (2005). Candida dubliniensis: ten years on. FEMS Microbiol Lett 253, 9-17.

Sundstrom, P. (1999). Adhesins in Candida albicans. Curr Opin Microbiol 2, 353-357.

Suter, B., Pogoutse, O., Guo, X., Krogan, N., Lewis, P., Greenblatt, J. F., Rine, J. \& Emili, A. (2007). Association with the origin recognition complex suggests a novel role for histone acetyltransferase Hat1p/Hat2p. BMC biology 5, 38.

Swoboda, R. K., Bertram, G., Delbruck, S., Ernst, J. F., Gow, N. A., Gooday, G. W. \& Brown, A. J. (1994). Fluctuations in glycolytic mRNA levels during morphogenesis in Candida albicans reflect underlying changes in growth and are not a response to cellular dimorphism. Mol Microbiol 13, 663-672.

Tebarth, B., Doedt, T., Krishnamurthy, S., Weide, M., Monterola, F., Dominguez, A. \& Ernst, J. F. (2003). Adaptation of the Efg1p morphogenetic pathway in Candida albicans by negative autoregulation and PKA-dependent repression of the EFG1 gene. J Mol Biol 329, 949962. 
Tekeli, A., Memikoglu, O., Dolapci, I., Koyuncu, E., Yuksel, O. \& Tekeli, E. (2005). The prevalence of Candida dubliniensis among germ tube positive candida samples isolated from the respiratory tract. Saudi Med J 26, 885-887.

Timpel, C., Strahl-Bolsinger, S., Ziegelbauer, K. \& Ernst, J. F. (1998). Multiple functions of Pmt1p-mediated protein O-mannosylation in the fungal pathogen Candida albicans. J Biol Chem 273, 20837-20846.

Timpel, C., Zink, S., Strahl-Bolsinger, S., Schroppel, K. \& Ernst, J. (2000). Morphogenesis, adhesive properties, and antifungal resistance depend on the Pmt6 protein mannosyltransferase in the fungal pathogen candida albicans. J Bacteriol 182, 3063-3071.

Tournu, H., Serneels, J. \& Van Dijck, P. (2005). Fungal pathogens research: novel and improved molecular approaches for the discovery of antifungal drug targets. Curr Drug Targets 6, 909-922.

Trofa, D., Gacser, A. \& Nosanchuk, J. D. (2008). Candida parapsilosis, an emerging fungal pathogen. Clin Microbiol Rev 21, 606-625.

Tzung, K. W., Williams, R. M., Scherer, S. \& other authors (2001). Genomic evidence for a complete sexual cycle in Candida albicans. Proc Natl Acad Sci U S A 98, 3249-3253.

Uhl, M. A., Biery, M., Craig, N. \& Johnson, A. D. (2003). Haploinsufficiency-based large-scale forward genetic analysis of filamentous growth in the diploid human fungal pathogen C.albicans. Embo J 22, 2668-2678.

Ushinsky, S. C., Harcus, D., Ash, J., Dignard, D., Marcil, A., Morchhauser, J., Thomas, D. Y., Whiteway, M. \& Leberer, E. (2002). CDC42 is required for polarized growth in human pathogen Candida albicans. Eukaryot Cell 1, 95-104.

Utley, R. T. \& Cote, J. (2003). The MYST family of histone acetyltransferases. Curr Top Microbiol Immunol 274, 203-236.

van het Hoog, M., Rast, T. J., Martchenko, M. \& other authors (2007). Assembly of the Candida albicans genome into sixteen supercontigs aligned on the eight chromosomes. Genome Biol 8, R52.

Varma, A., Singh, B. B., Karnani, N., Lichtenberg-Frate, H., Hofer, M., Magee, B. B. \& Prasad, R. (2000). Molecular cloning and functional characterisation of a glucose transporter, CaHGT1, of Candida albicans. FEMS Microbiol Lett 182, 15-21.

Vernarecci, S., Ornaghi, P., Bagu, A., Cundari, E., Ballario, P. \& Filetici, P. (2008). Gcn5p plays an important role in centromere kinetochore function in budding yeast. Mol Cell Biol $\mathbf{2 8}$, 988-996.

Verreault, A., Kaufman, P. D., Kobayashi, R. \& Stillman, B. (1998). Nucleosomal DNA regulates the core-histone-binding subunit of the human Hat1 acetyltransferase. Curr Biol 8, 96108.

Vidotto, V., Bruatto, M., Accattatis, G. \& Caramello, S. (1996). Observation on the nucleic acids in the chlamydospores of Candida albicans. New Microbiol 19, 327-334.

Vogelauer, M., Wu, J., Suka, N. \& Grunstein, M. (2000). Global histone acetylation and deacetylation in yeast. Nature 408, 495-498.

Walker, L. A., Maccallum, D. M., Bertram, G., Gow, N. A., Odds, F. C. \& Brown, A. J. (2009). Genome-wide analysis of Candida albicans gene expression patterns during infection of the mammalian kidney. Fungal Genet Biol 46, 210-219. 
Walsh, R. B., Kawasaki, G. \& Fraenkel, D. G. (1983). Cloning of genes that complement yeast hexokinase and glucokinase mutants. J Bacteriol 154, 1002-1004.

Wang, Y., Cao, Y. Y., Jia, X. M., Cao, Y. B., Gao, P. H., Fu, X. P., Ying, K., Chen, W. S. \& Jiang, Y. Y. (2006). Cap1p is involved in multiple pathways of oxidative stress response in Candida albicans. Free radical biology \& medicine 40, 1201-1209.

Wang, Y. L., Faiola, F., Xu, M., Pan, S. \& Martinez, E. (2008a). Human ATAC is a GCN5/PCAF-containing acetylase complex with a novel NC2-like histone fold module that interacts with the TATA-binding protein. J Biol Chem.

Warenda, A. J. \& Konopka, J. B. (2002). Septin function in Candida albicans morphogenesis. Mol Biol Cell 13, 2732-2746.

Weinstein, B. \& Solomon, F. (1990). Phenotypic consequences of tubulin overproduction in Saccharomyces cerevisiae: differences between alpha-tubulin and beta-tubulin. Mol Cell Biol $10,5295-5304$.

Wey, S. B., Mori, M., Pfaller, M. A., Woolson, R. F. \& Wenzel, R. P. (1988). Hospital-acquired candidemia. The attributable mortality and excess length of stay. Arch Intern Med 148, 26422645.

White, T. C. \& Agabian, N. (1995). Candida albicans secreted aspartyl proteinases: isoenzyme pattern is determined by cell type, and levels are determined by environmental factors. $J$ Bacteriol 177, 5215-5221.

Whiteway, M. \& Oberholzer, U. (2004). Candida morphogenesis and host-pathogen interactions. Curr Opin Microbiol 7, 350-357.

Wightman, R., Bates, S., Amornrrattanapan, P. \& Sudbery, P. (2004). In Candida albicans, the Nim1 kinases Gin4 and Hsl1 negatively regulate pseudohypha formation and Gin4 also controls septin organization. J Cell Biol 164, 581-591.

Wilson, W. A., Wang, Z. \& Roach, P. J. (2002). Systematic identification of the genes affecting glycogen storage in the yeast Saccharomyces cerevisiae: implication of the vacuole as a determinant of glycogen level. Mol Cell Proteomics 1, 232-242.

Wolffe, A. P. \& Guschin, D. (2000). Review: chromatin structural features and targets that regulate transcription. J Struct Biol 129, 102-122.

Wolyniak, M. J. \& Sundstrom, P. (2007). Role of actin cytoskeletal dynamics in activation of the cyclic AMP pathway and HWP1 gene expression in Candida albicans. Eukaryot Cell 6, 1824-1840.

Wu, J., Carmen, A. A., Kobayashi, R., Suka, N. \& Grunstein, M. (2001). HDA2 and HDA3 are related proteins that interact with and are essential for the activity of the yeast histone deacetylase HDA1. Proc Natl Acad Sci U S A 98, 4391-4396.

Xu, Z., Zhang, L. X., Zhang, J. D., Cao, Y. B., Yu, Y. Y., Wang, D. J., Ying, K., Chen, W. S. \& Jiang, Y. Y. (2006). cDNA microarray analysis of differential gene expression and regulation in clinically drug-resistant isolates of Candida albicans from bone marrow transplanted patients. Int $\checkmark$ Med Microbiol 296, 421-434.

Yaar, L., Mevarech, M. \& Koltin, Y. (1997). A Candida albicans RAS-related gene (CaRSR1) is involved in budding, cell morphogenesis and hypha development. Microbiology 143 ( Pt 9), 3033-3044. 
Yan, L., Zhang, J., Li, M., Cao, Y., Xu, Z., Cao, Y., Gao, P., Wang, Y. \& Jiang, Y. (2008). DNA microarray analysis of fluconazole resistance in a laboratory Candida albicans strain. Acta Biochim Biophys Sin (Shanghai) 40, 1048-1060.

Yang, C. W., Barkham, T. M., Chan, F. Y. \& Wang, Y. (2003). Prevalence of Candida species, including Candida dubliniensis, in Singapore. J Clin Microbiol 41, 472-474.

Yang, X. J. (2004). The diverse superfamily of lysine acetyltransferases and their roles in leukemia and other diseases. Nucleic Acids Res 32, 959-976.

Yang, X. J. \& Gregoire, S. (2005). Class II histone deacetylases: from sequence to function, regulation, and clinical implication. Mol Cell Biol 25, 2873-2884.

Yin, Z., Stead, D., Selway, L. \& other authors (2004). Proteomic response to amino acid starvation in Candida albicans and Saccharomyces cerevisiae. Proteomics 4, 2425-2436.

Yokoyama, K. \& Takeo, K. (1983). Differences of asymmetrical division between the pseudomycelial and yeast forms of Candida albicans and their effect on multiplication. Arch Microbiol 134, 251-253.

Yokoyama, K., Kaji, H., Nishimura, K. \& Miyaji, M. (1994). The role of microfilaments and microtubules during pH-regulated morphological transition in Candida albicans. Microbiology 140 ( Pt 2), 281-287.

Zakikhany, K., Naglik, J. R., Schmidt-Westhausen, A., Holland, G., Schaller, M. \& Hube, B. (2007). In vivo transcript profiling of Candida albicans identifies a gene essential for interepithelial dissemination. Cellular microbiology 9, 2938-2954.

Zeng, Y. B., Qian, Y. S., Ma, L. \& Gu, H. N. (2007). Genome-wide expression profiling of the response to terbinafine in Candida albicans using a cDNA microarray analysis. Chin Med $J$ (Engl) 120, 807-813.

Zhao, R., Daniels, K. J., Lockhart, S. R., Yeater, K. M., Hoyer, L. L. \& Soll, D. R. (2005a). Unique aspects of gene expression during Candida albicans mating and possible $G(1)$ dependency. Eukaryot Cell 4, 1175-1190.

Zhao, X., Pujol, C., Soll, D. R. \& Hoyer, L. L. (2003). Allelic variation in the contiguous loci encoding Candida albicans ALS5, ALS1 and ALS9. Microbiology 149, 2947-2960.

Zhao, X., Oh, S. H., Yeater, K. M. \& Hoyer, L. L. (2005b). Analysis of the Candida albicans Als $2 p$ and Als $4 p$ adhesins suggests the potential for compensatory function within the Als family. Microbiology 151, 1619-1630.

Zheng, X., Wang, Y. \& Wang, Y. (2004). Hgc1, a novel hypha-specific G1 cyclin-related protein regulates Candida albicans hyphal morphogenesis. Embo J 23, 1845-1856.

Znaidi, S., De Deken, X., Weber, S., Rigby, T., Nantel, A. \& Raymond, M. (2007). The zinc cluster transcription factor Tac1p regulates PDR16 expression in Candida albicans. Mol Microbio/ 66, 440-452. 